On segregation in bidisperse granular flows

Irana F. C. Denissen

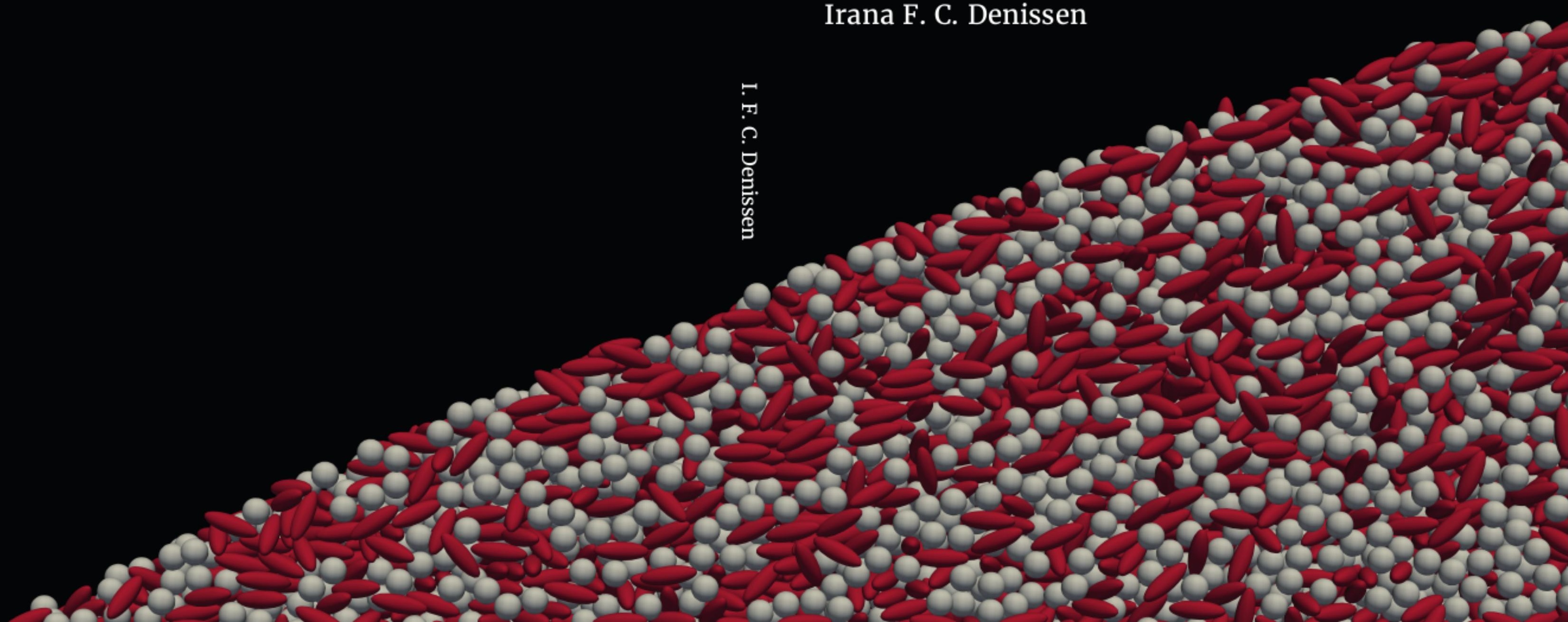




\title{
ON SEGREGATION IN BIDISPERSE GRANULAR FLOWS
}

\author{
Irana Francisca Catharina Denissen
}




\section{Thesis committee members:}

Chairman:

Prof. dr. G. P. M. R. Dewulf University of Twente

Promotor:

Prof. dr. A.R. Thornton

University of Twente

Co-promotor:

Dr. T. Weinhart

Universiteit Twente

Members:

prof. dr. R. Cruz Hidalgo Universidad de Navarra prof. dr. J. M. N. T. Gray University of Manchester dr. ir. W. K. den Otter University of Twente prof. dr. ir. C. H. Venner University of Twente dr. N. Vriend

University of Cambridge

The work in this thesis was carried out at the Multiscale Mechanics (MSM) group, MESA+ Institute of Nanotechnology, Faculty of Engineering Technology (ET), University of Twente, Enschede, The Netherlands.

This work was financially supported by NWO grant TTW-VIDI 13472.

Cover design: K. Dearo Garcia and I.F.C. Denissen

Copyright () 2019 by I.F.C. Denissen

Published by Ipskamp Printing, Enschede, The Netherlands

ISBN: 978-90-365-4876-2

DOI number: 10.3990/1.9789036548762

Official URL: https://doi.org/10.3990/1.9789036548762 


\title{
ON SEGREGATION IN BIDISPERSE GRANULAR FLOWS
}

\section{DISSERTATION}

\author{
to obtain \\ the degree of doctor at the University of Twente, \\ on the authority of the rector magnificus, \\ Prof.dr. T.T.M. Palstra, \\ on account of the decision of the Doctorate Board, \\ to be publicly defended \\ on Thursday the $28^{\text {th }}$ of November 2019 at 12:45
}

by

\author{
Irana Francisca Catharina Denissen \\ born on the $6^{\text {th }}$ of April 1990 \\ in Weststellingwerf, The Netherlands
}


This dissertation was approved by the promotor:

Prof. dr. A.R. Thornton

and the co-promotor:

dr. T. Weinhart 


\section{CONTEnTS}

Summary $\quad$ ix

Samenvatting $\quad$ xi

1 Introduction 1

1.1 Granular flows . . . . . . . . . . . . . . . . . . . . 1

1.2 Modelling and validation methods . . . . . . . . . . . . . . . . 2

1.3 Thesis outline. . . . . . . . . . . . . . . . . . 5

References...................... 6

2 Flow through a contraction 11

2.1 Introduction . . . . . . . . . . . . . . . 11

2.2 Asymptotic theory . . . . . . . . . . . . . . . . 13

2.2.1 Constitutive law/Closure relation . . . . . . . . . . . . . . . 15

2.2 .2 Steady state solutions . . . . . . . . . . . . . . . . . . . . . . . . . . . . 216

2.2 .3 Shock solutions . . . . . . . . . . . . . . . . . 21

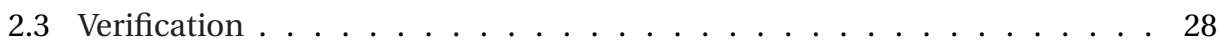

2.3.1 Two-dimensional DGFEM solutions . . . . . . . . . . . . . . . 29

2.3.2 Two-dimensional DGFEM vs. One-dimensional asymptotic theory . 29

2.4 Conclusions. . . . . . . . . . . . . . . . . . 33

2.4 .1 Summary . . . . . . . . . . . . . . 33

2.4 Future work . . . . . . . . . . . . . . . . 33

2.4 .3 Acknowledgements ................ 33

References . . . . . . . . . . . . . . . . 34

Appendix $\quad 39$

2.A Details of derivation 1D shallow granular model . . . . . . . . . . . . 39

2.A.1 Kinematic boundary conditions . . . . . . . . . . . . 39

2.A.2 Width-averaging. . . . . . . . . . . . . 39

2.A.3 Derived relations. . . . . . . . . . . . . . . . . . 41

2.A.4 Froude function . . . . . . . . . . . . . . . . . . . . . . . 41

2.A.5 Analytic solution for inviscid flow . . . . . . . . . . . . . 42

2.B Regularisation ... . . . . . . . . . . . . . . . . . . . . . . . . . 42

2.B.1 Inviscid flows $(\mu \approx \tan \theta) \ldots \ldots \ldots 42$

2.B.2 Frictional flows (Viscid) . . . . . . . . . . . . . . . . . 44

2.C Jump conditions . . . . . . . . . . . . . . . . . . 49

References...................... 50 
3 Bulbous head formation $\mathbf{5 1}$

3.1 Introduction . . . . . . . . . . . . . . . . . 51

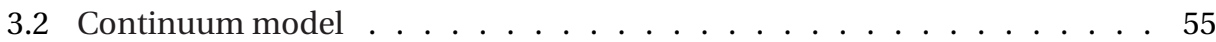

3.2.1 Definitions. . . . . . . . . . . . . . . 55

3.2 Assumptions. . . . . . . . . . . . . . . . 56

3.2 .3 System of equations . . . . . . . . . . . . . . . . . . . 57

3.2.4 Closure relations. . . . . . . . . . . . . . . . . . . . . . . . 59

3.3 Time-dependent numerical solution . . . . . . . . . . . . . . . 60

3.4 Travelling wave solution . . . . . . . . . . . . . . . . . . 62

3.4.1 Boundary conditions. . . . . . . . . . . . . . . . . 64

3.4.2 Shock properties. . . . . . . . . . . . . . . . . 65

3.4.3 ODE solution . . . . . . . . . . . . . . . . 65

3.4.4 Comparison with time-dependent solution . . . . . . . . . . . . 67

3.5 Comparison with discrete particle simulations . . . . . . . . . . . . . . 67

3.5.1 Height profiles . . . . . . . . . . . . . . . . . . . 68

3.5.2 Segregation profiles . . . . . . . . . . . . . . . 72

3.6 Conclusion and discussion . . . . . . . . . . . . . . . . . . . . . . . . . . . . . . . . . 72

References......................... 75

$\begin{array}{lc}\text { Appendix } & 83\end{array}$

3.A DGFEM discretisation. . . . . . . . . . . . . . . . . . 83

3.A.1 Notation . . . . . . . . . . . . . . . . 83

3.A.2 Discretisation .................. . . 83

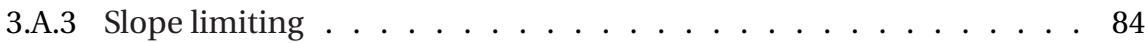

3.A.4 Wetting and drying treatment . . . . . . . . . . . . . . . . 84

3.A.5 Time integration . . . . . . . . . . . . . . . . . . 84

3.B Details of the travelling wave solution. . . . . . . . . . . . . . 85

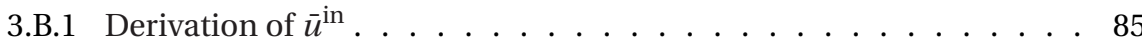

3.B.2 Conservation of large-particle volume . . . . . . . . . . . . . 86

3.C Details of discrete particle simulations . . . . . . . . . . . . . . 86

3.C.1 Definitions. . . . . . . . . . . . . . 86

3.C.2 Contact law . . . . . . . . . . . . . . . . 87

3.C.3 Dynamics . . . . . . . . . . . . . . . . . . . 88

3.C.4 Chute geometry . . . . . . . . . . . . . . . . 89

3.C.5 Maser inflow boundary condition . . . . . . . . . . . . . . . . 89

References...................... 90

4 Anisotropic coarse graining for shallow flows 93

4.1 Introduction . . . . . . . . . . . . . . . . . . 93

4.2 Method .............................. 94

4.2.1 Discrete particle simulation setup . . . . . . . . . . . . . 94

4.2.2 Anisotropic coarse-graining . . . . . . . . . . . . . . . . 95

4.2.3 Validation for shallow bidisperse flows. . . . . . . . . . . . 98

4.3 Application . . . . . . . . . . . . . . . . . 100

4.4 Conclusion and outlook. . . . . . . . . . . . . . . . . . 103

References........................ 103 
5 Segregation of ellipsoidal particles in a rotating drum $\quad 107$

5.1 Introduction . . . . . . . . . . . . . . . . . . . . . 107

5.2 Simulation method . . . . . . . . . . . . . . . . . 109

5.2.1 Discrete particle simulations. . . . . . . . . . . . . . . . 109

5.2 .2 Analysis . . . . . . . . . . . . . . . . . 110

5.3 Results . . . . . . . . . . . . . . . . . . 111

5.3.1 Segregation and inverse segregation . . . . . . . . . . . 111

5.3.2 Segregation index . . . . . . . . . . . . . . . . . 111

5.4 Conclusion and discussion . . . . . . . . . . . . . . . . . . . . . . . . 114

References........................ 115

Appendix $\quad \mathbf{1 1 9}$

5.A Implementation of superquadric particles in MercuryDPM. . . . . . . . . 119

5.A.1 Particle properties . . . . . . . . . . . . . . . . . . . . 119

5.A.2 Contact detection . . . . . . . . . . . . . . . . . . . 124

5.A.3 Force computation. . . . . . . . . . . . . . . 128

5.A.4 Outlook . . . . . . . . . . . . . . . . . 129

5.B Drum-algorithm . . . . . . . . . . . . . . . 130

5.B.1 Drum simulation. . . . . . . . . . . . . . . . . 130

5.B.2 Prescribed position and velocity . . . . . . . . . . . . . 131

References . . . . . . . . . . . . . . . . . 131

6 Conclusions and Outlook 133

References . . . . . . . . . . . . . . . . . . 135

$\begin{array}{ll}\text { Acknowledgements } & 137\end{array}$

Curriculum Vitae 139 


\section{SUMMARY}

Every year, thousands of people die due to landslides, avalanches and other natural disasters in which separate particles play an important role. For accurate zonation and risk assessment, efficient models are needed that not only take into account the basal topography, but also the properties of these particles (snow, rock, sand, etc.), such as sizeand shape-distribution. The conglomoration of particles is called a granular material, and the flow of a granular material is a granular flow.

When a granular material is composed of multiple components that differ in e.g. size, density or shape, the mixture usually segregates into separate phases when sheared or shaken. For example in a size-bidisperse flow over an inclined plane, which contains large and small particles, the larger particles tend to the free surface, while the small particles tend to the base. Both the cause of segregation and the phenomenological aspects are active areas of research. This thesis looks at the latter; it aims to develop efficient and accurate models of granular flows, in particular of bidisperse granular flows, which consist of two types of particles.

We start in chapter 2 by considering the flow of a monodisperse granular material down a rough inclined channel with downslope contracting sidewalls. Utilising a depthaveraged shallow granular theory together with an empirical constitutive friction law, an extended one-dimensional (depth- and width-averaged) granular hydraulic theory is presented. For a range of upstream flow conditions and channel openings, the onedimensional model predicts three different steady states, where the velocity and height of the flow do not change over time. The three states are: smooth subcritical flows, supercritical flows with weak oblique shocks (smooth when width-averaged) and flows with a steady jump in the contraction region. Both the super- and subcritical flow states are verified by numerically solving the depth-averaged two-dimensional shallow granular model. Despite the strong inhomogeneities in the contraction region, the onedimensional solutions match well with the two-dimensional solutions that are averaged across the channel.

Next, we consider size-bidisperse flows over a uniform, long, narrow channel, where the larger particles segregate towards the free surface. As the surface velocity of such flows is larger than the mean velocity, the larger material is transported to the flow front. This causes size-segregation in the downstream direction, resulting in a flow front composed of large particles. Since the large particles are often more frictional than the small, the mobility of the flow front is reduced, resulting in a so-called bulbous head; the flowfront is thicker than its tail. Chapter 3 focuses on the formation and evolution of this bulbous head, which is shown to emerge in both a depth-averaged continuum framework and discrete particle simulations. Furthermore, numerical solutions of the continuum model converge to a travelling wave solution, which allows for efficient computation of the long-time behaviour of the flow. Using small-scale periodic discrete particle simulations to calibrate (close) the continuum framework, the simple one-dimensional model 
is validated with full-scale three-dimensional discrete particle simulations. The comparison shows that there are conditions under which the model works surprisingly well given the strong approximations made; for example, instantaneous vertical segregation.

In order to compare discrete particle simulations with continuum fields, the discrete particle data needs to be mapped on these continuum fields. Chapter 4 shows a novel micro-macro technique called anisotropic coarse graining, which is able to obtain continuum fields from the discrete particle data with different length scales in different directions, while conserving both mass and momentum and being grid independent. This is especially helpful in shallow flows with elongated flow features, such as breaking segregation waves or subtle downslope segregation patterns. Applying the anisotropic coarse graining technique to bidisperse shallow granular flows over a rough channel, the resulting concentration fields show that microscopic friction of the particles is one cause of segregation in these flows. Furthermore, the sharpness and length of breaking segregation waves depends on the size ratio of the particles and height of the flow: the breaking segregation wave becomes sharper and narrower with increasing ratio of particle sizes and decreasing flow height.

Lastly, chapter 5 looks at the influence of particle elongation on segregation. Using a rotating drum to simulate binary mixtures of spheres and prolate ellipsoids of different aspect ratios, it shows that the segregation due to differences in aspect ratio is a complex phenomenon. Not only does the segregation change in strength, it also changes direction: for mixtures of spheres and prolate ellipsoids with a small aspect ratio, the ellipsoids segregate to the core of the flow. For mixtures of spheres and more elongated prolate ellipsoids on the other hand, the ellipsoids tend to segregate towards the outside. This is a smooth transition in segregation strength from no segregation (spheresphere) to a local maximum in segregation strength (sphere-short ellipsoids) towards inverse segregation (sphere-long ellipsoids).

All in all, this thesis has contributed to developing efficient and accurate models for segregating granular flows as follows: it has first shown that sometimes simple and efficient models are sufficient for predicting granular flows, followed by developing a tool to better analyse shallow granular flows and lastly it charts the segregation behaviour of mixtures with ellipsoidal particles. 


\section{SAMENVATTING}

Jaarlijks sterven er duizenden mensen door lawines en andere natuurrampen waar losse stenen, zand en sneeuw een belangrijke rol spelen. Om deze lawines goed te kunnen voorspellen is het belangrijk om niet alleen de topografie van de helling, maar ook de eigenschappen van de losse deeltjes mee te nemen, zoals bijvoorbeeld hun grootte en vorm. De groep deeltjes als geheel noemen we een granulaat, en de stroming van deze deeltjes een granulaire stroming.

Als de deeltjes uit een granulaat van elkaar verschillen in bepaalde eigenschappen, zoals in grootte, vorm of dichtheid, dan segregeert (ontmengt) dit granulaat als deze van een helling stroomt, wordt rondgedraaid in een mixer of wordt geschud. Als we bijvoorbeeld een mengsel van grote en kleine deeltjes nemen en die van een helling af laten stromen, dan komen de grote deeltjes aan de oppervlakte en zakken de kleine deeltjes naar de bodem. Op dit moment gebeurt er veel onderzoek naar zowel de oorzaak als de fenomenologische aspecten van deze segregatie. In dit proefschrift kijken we naar dit laatste; het doel is om efficiënte en accurate modellen voor granulaire stromingen te ontwikkelen, met name voor granulaire stromingen die uit twee verschillende soorten deeltjes bestaan (bidisperse granulaire stromingen).

In hoofdstuk 2 wordt eerst gekeken naar monodisperse granulaire stromingen over een helling met een versmalling aan het einde. Er is hiervoor een eenvoudig model voor de hoogte en snelheid van de stroming afgeleid vanuit een bestaand model. De evenwichtsoplossingen van het model blijken afhankelijk te zijn van de breedte van de versmalling en de hoogte en snelheid van de instroom. Het eenvoudige model voorspelt wanneer er jumps of shocks optreden, en de oplossingen van dit eenvoudige model komen goed overeen met de oplossingen van het originele model.

In de rest van dit proefschrift kijken we naar bidisperse stromingen, waarin het granulaat bestaat uit twee soorten deeltjes. Als we een mengsel van grote en kleine deeltjes van een helling laten stromen, dan gaan de grote deeltjes naar de oppervlakte en zakken de kleine deeltjes naar de bodem. Aangezien de snelheid van de stroming aan het oppervlak hoger is dan bij de bodem, betekent dit dat zich aan de voorkant van de stroming meer grote deeltjes bevinden dan kleine; er is zowel een horizontaal als verticaal segregatieprofiel zichtbaar. Als dan bovendien de grote deeltjes ook meer wrijving met de bodem hebben, dan zien we dat aan de voorkant er meer dissipatie van energie is dan in de staart, en dat dus de deeltjes ophopen en de voorkant hoger wordt dan de staart; dit fenomeen noemen wij de bulbous head. In hoofdstuk 3 tonen we aan dat deze bulbous head gereproduceerd kan worden met zowel een discrete deeltjesmethode als een simpel eendimensionaal continumodel in termen van hoogte, snelheid en gemiddelde concentratie kleine deeltjes van de stroming. We zien dat numerieke oplossingen van het continumodel convergeren naar een oplossing met constante vorm en snelheid. Bovendien zijn er omstandigheden waaronder het continumodel verbazingwekkend goed overeen komt met de discrete deeltjessimulaties, ondanks de zeer sterke aannames die 
zijn gemaakt in het continumodel.

Om de data van discrete deeltjessimulaties te vergelijken met continumodellen wordt coarse graining gebruikt. In hoofdstuk 4 is deze techniek zodanig uitgebreid dat deze om kan gaan met verschillende lengteschalen in verschillende richtingen. Deze anisotropische coarse graining techniek is met name handig als de granulaire stromingen inherent verschillende lengteschalen hebben. Als we deze techniek toepassen op bidisperse granulaire stromingen van een helling, dan zien we dat oppervlakteruwheid een oorzaak is van segregatie. De lengte en scherpte van het segregatiepatroon (breaking segregation wave) hangt bovendien sterk af van de verhouding van de grootte van de deeltjes: hoe groter deze verhouding, hoe korter en scherper de breaking segregation wave.

Ten slotte kijken we in hoofdstuk 5 naar de segregatie op basis van aspect ratio (beeldverhouding) van de deeltjes. Dit doen we in een horizontale, ronddraaiende cilinder met daarin mengsels van bollen en prolate ellipsoïden van verschillende lengtes, maar constant volume. Hieruit blijkt dat segregatie op basis van aspect ratio een complex fenomeen is: als de ellipsoïden relatief kort zijn, dan segregeren de bollen naar de buitenkant van de cilinder en de ellipsoïden naar de kern. Aan de andere kant, als de ellipsoïden relatief lang zijn, dan segregeren de bollen naar de kern en de ellipsoïden naar de buitenkant.

Alles bij elkaar zijn er in dit proefschrift stappen gezet richting efficiënte en accurate modellen voor granulaire stromingen: eerst is aangetoond dat in sommige gevallen zeer simpele modellen voldoen bij het voorspellen van granulaire stromingen, vervolgens is er gereedschap ontwikkeld om deze stromingen beter te kunnen analyseren en ten slotte is het segregatiegedrag van ellipsoïden in kaart gebracht. 


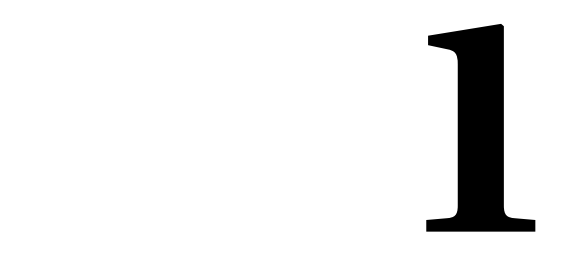

INTRODUCTION

\subsection{Granular FlOWS}

Granular materials consist of macroscopic particles that dissipate energy when the particles interact. You can find them everywhere in your daily life; for an example take a look into your pantry: flour, sugar, rice and many other dry goods are types of granular materials (figure 1.1). While these pantry items behave like a solid when at rest, they flow similar to a fluid when they are being poured out of their containers. There are many other instances of granular flows, both in nature and industry, such as avalanches, landslides, conveyor belts, and industrial mixers. Granular flows are usually temporary phenomena: unlike classical fluids, granular flows dissipate energy with every collision and thus settle into a solid state unless there is sufficient energy provided by external agitation. This is why a pile of sand forms at the bottom of an hourglass, and why avalanches do not flow below a certain inclination. The energy needed for grains to flow can either be provided at the beginning (e.g. flipping an hourglass or tilting a food container), continuously (e.g. rotating a concrete mixer), or a combination thereof.

Granular materials are sometimes monodisperse, meaning that all particles have the same properties, but usually the particles are different from each other in one or more properties, such as size, shape and density. Whenever granular materials flow, there is a tendency for particles with different properties to separate in the respective constituents: the flow segregates. In gravity-driven shear flows, the most potent (and most studied) type of segregation is size segregation, where the smaller particles tend to the bottom of the flow on an inclined plane, while the larger particles tend to the top. This can be seen in home experiments as well, for example when pouring out granola or making a crumble topping for a pie. Particles segregate strongly even at small size ratios [1], in a large variety of geometries, see for example figure 1.2 for some laboratory experiments in which segregation takes place. Segregation is usually unwanted in industrial processes, where one would want the material to be mixed as homogeneous as possible. The pharmaceutical industry, for instance, requires each tablet to have the same ratio of active and filler ingredients. To prevent size segregation during the tabletting pro- 


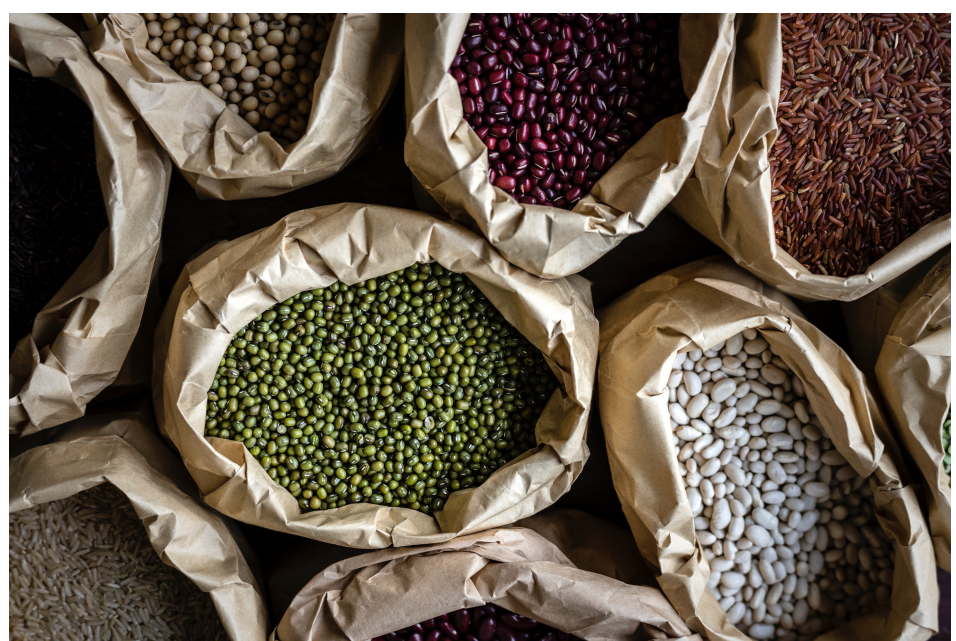

Figure 1.1: Granular materials come in many sizes and shapes, for example as beans, rice and other food stuffs.

cess, the particle size distribution is often narrowed beforehand by milling, granulation and/or sieving, and shearing motion is avoided as much as possible.

Determining the mechanism(s) that cause segregation in granular materials is an active area of research; most likely it is a combination of mechanisms dominating in different granular systems. In the dense granular flows over an inclined plane that we are interested in, the combination of kinetic sieving and squeeze expulsion is often used to describe size segregation [2-4]. In this description, segregation is caused by void spaces that open up between particles, allowing for percolation downwards. Small particles are more likely to fall into these gaps, which leads to a movement of small particles towards the base of the flow. At the same time, force imbalances squeeze all particles out of their layers towards the free surface, which leads to an upwards movement. The combination results in a net migration of small particles to the base and large particles towards the free surface. More recently, alternative mechanisms for size segregation, in dense chute flows, have been proposed, based on e.g. buoyancy effects [5, 6], differences in fluctuating kinetic energy [7, 8] and kinetic theory [9]. For shape segregation, a difference in the flowability also seems to play a large role $[10,11]$, as well as alignment of particles to minimise potential energy [12-16].

\subsection{MOdElling AND VALIDATION METHODS}

In order to test and validate models for the segregation and flow of granular materials, experimentation is a valuable tool. Segregation has been observed and measured in many different experimental setups, for example for flows over inclined planes or in rotating drums, e.g. $[1,3,21,22]$. There is currently no experimental method available that can measure the (flow) properties of all particles in granular flows, but various methods can measure specific properties of the flow. For example, optical measurements like Particle Image Velocimetry (PIV) or Particle Tracking Velocimetry (PTV) can be applied 

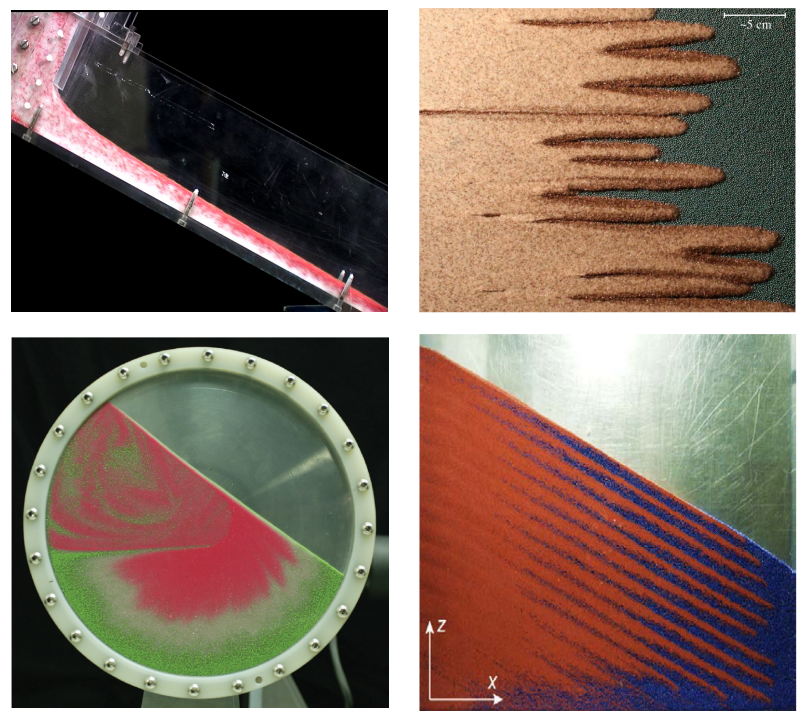

Figure 1.2: Experimental examples of segregation in granular flows in various geometries. Top left: quasi-2D narrow inclined plane [17], top right: inclined plane [18], bottom left: rotating drum [19], bottom right: quasi2D bounded heap [20].

to measure the properties of the particles visible on the flow surface $[23,24]$. In order to measure (segregation) properties of the entire flow, other methods need to be employed. For a simple flow over an inclined plane, splitter plates can be placed at the outflow in order to measure the number of particles at each height, and thus gives information about segregation behaviour [3]. For rotating drums, there are some experimental techniques available to look inside the flow, such as Positron Emitting Particle Tracking (PEPT), where the path of one particle can be tracked in a lot of detail [22, 25], Refractive Index-matched Scanning (RIMS), where the interior of the flow can be visualised after it has been paused [26], or cutting slices of the flow after it has been paused and solidified with wax [27].

To circumvent the limitations of the various experimental methods, one can use simulation techniques. Unlike experiments, simulations are data-rich and less timeconsuming. It is therefore much easier to simulate over a wide range of parameters, and because of the controlled environment, the influence of changing parameters can be studied more reliably. The most natural way to simulate granular materials is to use a particle-based method, where each physical particle is represented by a numerical particle. The most commonly used method in this is the discrete particle method (also called discrete element method) $[28,29]$, where the positions and velocities of each particle are computed at every time step. To account for the interactions between particles, contact laws are defined and evaluated to compute the forces and torques for each contact. These forces are used to update velocity and position of each particle at each time step in accordance to Newton's second law; similarly, torques determine angular velocity and orientation. For example, the left of figure 1.3 is a visualisation of discrete particle sim- 
ulations of a bidisperse flow over a rough inclined plane. Other particle-based simulation methods for granular materials exist. Non-smooth contact dynamics methods use a hard-sphere model where particles cannot overlap, while still using a time stepping scheme. They are suitable for quasi-static granular systems, but lack the flexibility in force-laws compared to discrete particle methods [30]. Lastly, event driven simulation methods also use the hard-sphere model, but do not use fixed time steps. Instead, time jumps from one collision to the next. This is efficient in sparse, gaseous like systems, but does not perform well in dense systems with many contacts [31]. For an overview of particle-based simulation techniques for granular flows, we refer the interested reader to [32].

Particle-based methods are accurate, but also computationally expensive. Most realworld processes contain billions or even trillions of particles and need to be studied for long time-intervals, making it practically impossible to simulate them with particlebased methods. Furthermore, the properties of individual particles are often not known, nor is it necessary to know them: For most applications, we are interested in bulk properties such as density and flow velocity. Therefore, large-scale systems are usually modelled by describing the behaviour of the bulk properties only: starting with the conservation of mass and momentum, systems of partial differential equations in terms of density, averaged velocity and stress can be derived to describe granular flows [33, 34]. These models typically are similar to their fluid equivalents, with the most notable change the term that accounts for the rheology [34-38]. Since we are interested in shallow granular flows that are typically much longer than they are deep, we realise that we can reduce computation time even more by using depth-averaged models. These typically are a system of partial differential equations in terms of the height and depth-averaged velocity of the flow [33], with constitutive models to incorporate the velocity profile [36, 37, 39]. Similarly, segregation behaviour can be captured by continuum models based on the conservation of mass and momentum for each type [20, 40-43]. In shallow flows, additional assumptions can be made for the long-time segregation behaviour and the equations can again be depth-averaged [44-46]. Lastly, (depth-averaged) segregation models can be coupled to (monodisperse) granular flow models, for example by providing feedback of the mixture composition in the rheology term of the flow $[47,48]$. See for example the right of figure 1.3, where the depth-averaged model of chapter 3 is used.

While (depth-averaged) continuum models are efficient, one needs to test their accuracy. That is, one should validate them to ensure that the assumptions that were made are not too strong for the given system. In order to compare continuum models with particle-based data, this discrete data must be somehow mapped onto continuum fields. A naive way is to divide the computational domain into equal-sized volumes, and compute the bulk density, velocity etc. of the granular material in each of these volumes. The main drawback of this method is that it leads to discrete values of these quantities instead of fields, where the resolution has to be sufficiently low that there are enough particles in each volume. Instead, one could use the coarse graining technique [39, 49] to go from discrete particle data to continuum fields. For each particle, a smooth kernel function is defined that is centered around the particle, for example a Gaussian function. Based on this one particle, macroscopic fields for density and momentum can be defined by multiplying the smoothing kernel with the (microscopic) density and momentum of 

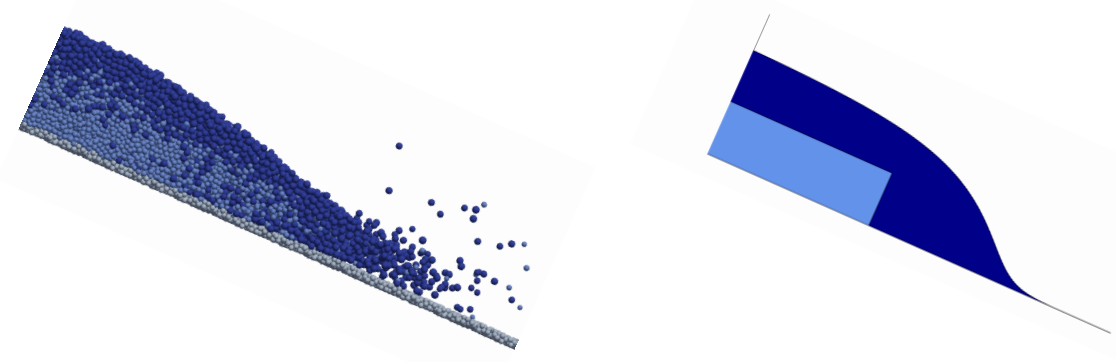

Figure 1.3: Example of simulation methods for bidisperse granular flows over an inclined plane. Left: discrete particle simulations, where every particle is represented by a sphere. Right: numerical solution of the continuum model in chapter 3, where only the height, velocity and small-particle concentration of the flow are modelled. In both cases, light blue represents the small particles, and dark blue represents the large particles.

the particle. Similarly, each particle and each contact of particles gives rise to a macroscopic stress field. Summing the contributions of all particles and contacts, this results in density, momentum and stress fields that exactly satisfy the conservation of mass and momentum [39, 49]. The conservation of mass and momentum is independent of the number of particles in the material (it already works for just one particle) and also works for each component of mixtures of different kinds of particles [43]. Since coarse graining results in continuum fields and not discrete points, comparison with continuum models can be done at the points that are convenient for the numerical method used, such as the volume centres when using a finite volume method, or the Gauss quadrature points when using a (discrete) Galerkin finite element method. Therefore, this method is very suitable to go from discrete particle data to continuum fields and is used throughout this thesis.

\subsection{THESIS OUTLINE}

The main goal of this thesis is to develop efficient and accurate models of (segregating) granular flows, in order to predict their behaviour more effectively. To do so, we use a combination of the methods discussed above: discrete particle simulations and coarse graining to study the qualitative and quantitative behaviour of granular flows, which are then also used to close and validate the more efficient continuum models.

To start with, chapter 2 looks at an established depth-averaged continuum model for monodisperse flows over inclined planes, and uses width-averaging to reduce the complexity of the model. The depth- and width-averaged model is tested for shallow flows over an inclined plane with a linear contraction, where the depth-averaged model is compared with the depth- and width-averaged model. Furthermore, simple expressions for steady flows are derived, where the behaviour of the flow solely depends on the height and Froude number at the inflow.

Chapter 3 also uses the simple depth-averaged continuum models, but studies shallow bidisperse flows in a narrow channel. For these flows, it is experimentally known that 
they develop a bulbous head, where the front of the flow is thicker than its tail [50,51]. The goal of this chapter is to reproduce the behaviour of the bulbous head with a variety of simulation methods. In order to do this, a novel depth-averaged model is developed for the height, velocity and particle concentration. Using this model, a discontinuous Galerkin finite element method is used to show the formation of a bulbous head over time. For the same model, a novel travelling wave solution is derived in order to predict long-time behaviour, and the matching of this travelling wave solution with an existing travelling wave solution [52] is compared to the numerical results. Lastly, discrete particle simulations are performed to compare the continuum model against.

In order to properly compare discrete particle models with continuum models for elongated flows, the coarse graining technique is extended in chapter 4 . An anisotropic coarse graining method is developed that can deal with different length scales in different directions, so that variations in the slow direction can be seen more clearly. This method is then utilised to examine the influence of size ratio on shallow flows over rough inclined planes.

While difference in size is one of the most important causes of segregation, the shape of the particles also plays a role. Therefore, in chapter 5 the behaviour of segregation in flows of spheres and ellipsoids is studied. Mixtures of spheres and prolate ellipsoids of various aspect ratios are simulated in a rotating cylinder, so that the influence of the elongation of ellipsoids on segregation can be studied.

Lastly, chapter 6 provides a summary of the conclusions of the aforementioned chapters is given, as well as the outlook for future work.

\section{REFERENCES}

[1] S. Wiederseiner, N. Andreini, G. Épely-Chauvin, G. Moser, M. Monnereau, J. M. N. T. Gray, and C. Ancey, Experimental investigation into segregating granular flows down chutes, Physics of Fluids 23, 013301 (2011).

[2] G. V. Middleton, Experimental Studies Related to Problems of Flysch Sedimentation,, Vol. 7 (The Geological Association of Canada, 1970) pp. 253-272.

[3] S. B. Savage and C. K. K. Lun, Particle size segregation in inclined chute flow of dry cohesionless granular solids, Journal of Fluid Mechanics 189, 311 (1988).

[4] J. W. Vallance and S. B. Savage, Particle segregation in granular flows down chutes, in IUTAM Symposium on Segregation in Granular Flows (Springer, 2000) pp. 31-51.

[5] D. V. Khakhar, J. J. McCarthy, and J. M. Ottino, Mixing and segregation of granular materials in chute flows, Chaos: An Interdisciplinary Journal of Nonlinear Science 9, 594 (1999).

[6] G. Pereira, M. Sinnott, P. Cleary, K. Liffman, G. Metcalfe, and I. Šutalo, Insights from simulations into mechanisms for density segregation of granular mixtures in rotating cylinders, Granular Matter 13, 53 (2011).

[7] Y. Fan and K. M. Hill, Theory for shear-induced segregation of dense granular mixtures, New journal of physics 13, 095009 (2011). 
[8] L. Staron and J. C. Phillips, Stress partition and microstructure in size-segregating granular flows, Phys. Rev. E 92, 022210 (2015).

[9] M. Larcher and J. T. Jenkins, Segregation and mixture profiles in dense, inclined flows of two types of spheres, Physics of Fluids 25, 113301 (2013).

[10] G. Pereira and P. Cleary, Segregation due to particle shape of a granular mixture in a slowly rotating tumbler, Granular Matter 19, 23 (2017).

[11] S. Y. He, J. Q. Gan, D. Pinson, and Z. Y. Zhou, Particle shape-induced radial segregation of binary mixtures in a rotating drum, Powder Technology (2018).

[12] C. R. A. Abreu, F. W. Tavares, and M. Castier, Influence of particle shape on the packing and on the segregation of spherocylinders via monte carlo simulations, Powder Technology 134, 167 (2003).

[13] C. R. K. Windows-Yule, B. J. Scheper, W. K. den Otter, D. J. Parker, and A. R. Thornton, Modifying self-assembly and species separation in three-dimensional systems of shape-anisotropic particles, Physical Review E 93, 020901 (2016).

[14] B. J. Scheper, G. A. S. Alan, A. R. Thornton, and W. K. Den Otter, Segregation of nonspherical particles in narrow rotating drums, ITO conference, University of Twente (2014).

[15] B. J. Scheper, C. R. K. Windows-Yule, W. K. Den Otter, and A. R. Thornton, Comparison of beds of spherical and spherocylindrical particles in vibrofluidised and rotating drum experiments, WCCM/APCOM Seoul (2016).

[16] M. Alizadeh, A. Hassanpour, M. Pasha, M. Ghadiri, and A. Bayly, The effect of particle shape on predicted segregation in binary powder mixtures, Powder technology 319, 313 (2017).

[17] A. Thornton, A Study of Segregation in Granular Gravity Driven Free Surface Flows., Ph.D. thesis, The University of Manchester (2005).

[18] J. L. Baker, C. G. Johnson, and J. M. N. T. Gray, Segregation-induced finger formation in granular free-surface flows, Journal of Fluid Mechanics 809, 168 (2016).

[19] J. M. N. T. Gray and C. Ancey, Multi-component particle-size segregation in shallow granular avalanches, Journal of Fluid Mechanics 678, 535 (2011).

[20] R. M. Lueptow, Z. Deng, H. Xiao, and P. B. Umbanhowar, Modeling segregation in modulated granular flow, in EPJ Web of Conferences, Vol. 140 (EDP Sciences, 2017) p. 03018.

[21] M. M. H. D. Arntz, H. H. Beeftink, W. K. den Otter, W. J. Briels, and R. M. Boom, Segregation of granular particles by mass, radius, and density in a horizontal rotating drum, AIChE journal 60, 50 (2014). 
[22] C. R. K. Windows-Yule, B. J. Scheper, A. J. van der Horn, N. Hainsworth, J. Saunders, D. J. Parker, and A. R. Thornton, Understanding and exploiting competing segregation mechanisms in horizontally rotated granular media, New journal of physics $\mathbf{1 8}$, 023013 (2016).

[23] W. Eckart and J. M. N. T. Gray, Particle image velocimetry (piv) for granular avalanches on inclined planes, in Dynamic Response of Granular and Porous Materials under Large and Catastrophic Deformations (Springer, 2003) pp. 195-218.

[24] N. Jesuthasan, B. R. Baliga, and S. B. Savage, Use of particle tracking velocimetry for measurements of granular flows: review and application, KONA Powder and Particle Journal 24, 15 (2006).

[25] D. J. Parker, C. J. Broadbent, P. Fowles, M. R. Hawkesworth, and P. McNeil, Positron emission particle tracking-a technique for studying flow within engineering equipment, Nuclear Instruments and Methods in Physics Research Section A: Accelerators, Spectrometers, Detectors and Associated Equipment 326, 592 (1993).

[26] S. Wiederseiner, N. Andreini, G. Epely-Chauvin, and C. Ancey, Refractive-index and density matching in concentrated particle suspensions: a review, Experiments in fluids 50, 1183 (2011).

[27] N. Dijkhuis and K. Schasfoort, Investigation of granular segregation in a rotating drum with a core, ITO conference, University of Twente (2015).

[28] P. A. Cundall and O. D. L. Strack, A discrete numerical model for granular assemblies, Geotechnique 29, 47 (1979).

[29] S. Luding, Introduction to discrete element methods: basic of contact force models and how to perform the micro-macro transition to continuum theory, European Journal of Environmental and Civil Engineering 12, 785 (2008).

[30] F. Radjai, The contact dynamics (cd) method, ALERT Doctoral School 2017 Discrete Element Modeling , 43 (2017).

[31] S. Luding, N. Rivas, and T. Weinhart, From soft and hard particle simulations to continuum theory for granular flows, ALERT geomaterials Doctoral School (2017).

[32] K. Taghizadeh, G. Combe, and S. Luding, ALERT Doctoral School 2017 Discrete Element Modeling, (2017).

[33] S. B. Savage and K. Hutter, The motion of a finite mass of granular material down a rough incline, Journal of fluid mechanics 199, 177 (1989).

[34] K. Hutter and K. R. Rajagopal, On flows of granular materials, Continuum Mechanics and Thermodynamics 6, 81 (1994).

[35] O. Pouliquen, Scaling laws in granular flows down rough inclined planes, Physics of Fluids 11, 542 (1999). 
[36] L. E. Silbert, D. Ertaş, G. S. Grest, T. C. Halsey, D. Levine, and S. J. Plimpton, Granular flow down an inclined plane: Bagnold scaling and rheology, Physical Review E 64, 051302 (2001).

[37] GDR-MiDi, On dense granular flows, The European Physical Journal E 14, 341 (2004).

[38] P. Jop, Y. Forterre, and O. Pouliquen, A constitutive law for dense granular flows, Nature 441, 727 (2006).

[39] T. Weinhart, A. R. Thornton, S. Luding, and O. Bokhove, Closure relations for shallow granular flows from particle simulations, Granular Matter 14, 531 (2012).

[40] J. M. N. T. Gray and A. R. Thornton, A theory for particle size segregation in shallow granular free-surface flows, Proceedings of the Royal Society of London A: Mathematical, Physical and Engineering Sciences 461, 1447 (2005).

[41] J. M. N. T. . Gray and V. A. Chugunov, Particle-size segregation and diffusive remixing in shallow granular avalanches, Journal of Fluid Mechanics 569, 365 (2006).

[42] B. Marks, P. Rognon, and I. Einav, Grainsize dynamics of polydisperse granular segregation down inclined planes, Journal of Fluid Mechanics 690, 499 (2012).

[43] D. R. Tunuguntla, A. R. Thornton, and T. Weinhart, From discrete elements to continuum fields: Extension to bidisperse systems, Computational particle mechanics 3, 349 (2016).

[44] J. M. N. T. Gray and B. P. Kokelaar, Large particle segregation, transport and accumulation in granular free-surface flows, Journal of Fluid Mechanics 652, 105 (2010).

[45] J. M. N. T. Gray and B. P. Kokelaar, Large particle segregation, transport and accumulation in granular free-surface flows-erratum, Journal of Fluid Mechanics 657, 539 (2010).

[46] A. N. Edwards and N. M. Vriend, Size segregation in a granular bore, Physical Review Fluids 1, 064201 (2016).

[47] M. J. Woodhouse, A. R. Thornton, C. G. Johnson, B. P. Kokelaar, and J. M. N. T. Gray, Segregation-induced fingering instabilities in granular free-surface flows, Journal of Fluid Mechanics 709, 543 (2012).

[48] I. F. C. Denissen, T. Weinhart, A. Te Voortwis, S. Luding, J. M. N. T. Gray, and A. R. Thornton, Bulbous head formation in bidisperse shallow granular flow over an inclined plane, Journal of Fluid Mechanics 866, 263 (2019).

[49] I. Goldhirsch, Stress, stress asymmetry and couple stress: from discrete particles to continuous fields, Granular Matter 12, 239 (2010).

[50] R. M. Iverson, M. Logan, R. G. LaHusen, and M. Berti, The perfect debris flow? Aggregated results from 28 large-scale experiments, Journal of Geophysical Research: Earth Surface 115 (2010). 
[51] B. P. Kokelaar, R. L. Graham, J. M. N. T. Gray, and J. W. Vallance, Fine-grained linings of leveed channels facilitate runout of granular flows, Earth and Planetary Science Letters 385, 172 (2014).

[52] G. Saingier, S. Deboeuf, and P.-Y. Lagrée, On the front shape of an inertial granular flow down a rough incline, Physics of Fluids 28, 053302 (2016). 


\section{2}

\section{GRANULAR FLOW DOWN A ROUGH INCLINED CHANNEL WITH A LINEAR CONTRACTION}

We consider a monodisperse dry granular material flowing down a rough inclined channel with downslope contracting sidewalls: theoretically and numerically. Utilising the depth-averaged shallow granular theory together with an empirical, but discrete particle simulations validated constitutive friction law, an extended novel one-dimensional (depth-and width-averaged) granular hydraulic theory is presented. For steady flows, besides describing the subcritical flow state, the one-dimensional model also predicts two other steady states, for a range of upstream prescribed flow conditions and channel openings. These states are supercritical flows with weak oblique shocks (smooth when widthaveraged) and flows with a steady jump in the contraction region. Both, super-and subcritical flow states were verified by numerically solving the depth-averaged two-dimensional shallow granular model. Despite the strong inhomogeneities in the linear contraction region, the one-and two-dimensional solutions (averaged across the channel) incomparably match well.

\subsection{INTRODUCTION}

A considerable number of industrial processes involve transport and handling of raw materials in a granular form, where grains of dissimilar properties are fed, mixed or separated. For example, partially filled rotating drums and blenders are utilised in pharmaceutical and food production industries [3] because of their mixing abilities and batch operations, whereas rotary kilns [4] and inclined channels [5] are employed for their ability to feed the material continuously. However, bulk handling devices are notorious for

This chapter is largely based on chapter 2 of the thesis of Tunuguntla [1]. Here, the main results are qualitative different because of a mistake in the original work, and more two-dimensional solutions are added for validation of the results. In preparation for publication [2]. 
their inefficiency. To improve their efficiency, industries either utilise a trial-and-error approach or discrete particle method simulations (DPMs). Although particle simulations are information-rich and give invaluable details, large-scale DPMs are known to be computationally expensive whereas the trial-and-error approach is time-inefficient and economically disadvantageous. Hence, the need for an economically viable timeefficient alternative such as continuum approach.

As a stepping stone towards developing powerful continuum formulations for complex granular phenomena, this work presents a time-efficient one-dimensional novel theory for investigating dense, rapid, free-surface granular flows in inclined channels [e.g. $6,7]$, utilising an existing depth-averaged shallow granular theory.

A majority of inclined-channel based granular flows in nature (avalanches, landslides, etc.) and industries are shallow, i.e. the ratio of the characteristic length scales in the normal $(H)$ to the streamwise direction $(L)$ is small, $H / L \ll 1$. By exploiting this shallowness aspect, several avalanche models have proved to be quite successful in quantitatively analysing inclined-channel granular flows [e.g. 7, 8]. In essence, such an avalanche model utilises the already existing shallow water theory from the fluids community and extends it to model the shallow granular free-surface flows. However, to do so, one still needs to define the corresponding granular constitutive relations (also known as the friction law) to relate the normal and the tangential stresses.

To our knowledge, the earliest known extension of the shallow water theory for modelling granular flows was put forward by Grigorian et al. [9], which they utilised to predict the snow avalanche paths in the Ural mountains. However, the formal existence of shallow granular (SG) theory was established by Savage and Hutter [10], who averaged the mass and momentum balance equations in the depth-direction and assumed a MohrCoulomb rheology with a constant Coulomb basal friction law. On depth-averaging, one averages out the depth-dependency from the flow quantities, such as the density and velocity [e.g. 11-13]. As years progressed, several researchers have utilised the SG theory to model various granular flow applications [e.g. 14-17]. Here, we focus on utilising the SG theory to effectively model granular flows through rough inclined channels with a linear contraction, also referred to as downslope deflecting walls. This configuration has previously been investigated by Hákonardóttir and Hogg [18], Vreman et al. [19], Cui et al. [20], Gray and Cui [21], Rhebergen et al. [22], Hogg and Jóhannesson [23].

Vreman et al. [19] theoretically and experimentally investigated the dynamics of granular flows in a smooth inclined channel with a linear contraction. Their experimental observations revealed, both, smooth flows with weak oblique shocks in the channel (supercritical state) and flows with steady jumps in the linear contraction region (reservoir state), which were also predicted via their one-dimensional granular 'hydraulic' theory. However, for closure, they related the normal and tangential stresses using the integrals of acceleration.

In this work, we will include the frictional effects by utilising an empirically determined friction law by Pouliquen and Forterre [24], which was further investigated and validated utilising DPMs [25, 26]. DPMs enable one to construct and calibrate a mapping between the micro-scale particle properties and macro-scale continuum quantities, allowing to determine the closure laws/constitutive relations required for any granular continuum model. As a result, Weinhart et al. [25] utilised an accurate micro-macro 
(a)

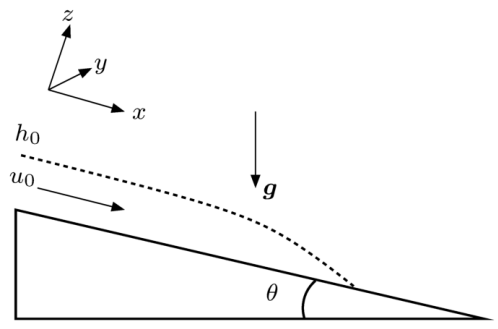

(b)

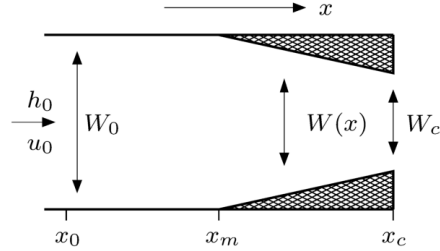

Figure 2.1: (a) Schematic of a granular flow down an inclined channel. (b) Top view of the channel with symmetric sidewall geometry and a linear contraction starting at $x_{m}$ and ending at $x_{c}$. Variables $h_{0}, u_{0}$ and $W_{0}$ denote the upstream flow depth, velocity and channel width, whereas $W_{c}$ denotes the exit channel width.

mapping technique called coarse-graining [27-29] to calibrate the friction law of Pouliquen and Forterre [24]. The same closure/friction law has now been extended to characterise self-channelising unconfined flows [30], varying basal properties [26] and bidisperse mixtures [31]. Thus enabling one to predict realistic flow scenarios using reduced continuum models, as shown in this study.

An efficient continuum model to predict the dynamics of a flowing bi- or polydisperse mixture down an inclined channel, would be a closed SG model combined with a particle segregation model [e.g. 32-34]. For a comprehensive review of the available particle segregation models, see Tunuguntla et al. [35]. However, this work focusses on efficiently predicting the dynamics of monodisperse granular material flowing in a channel with deflecting sidewalls, utilising a novel closure for the one-dimensional shallow granular model.

Through our one-dimensional granular hydraulic model, the flow regimes observed for a flow in an inclined channel with linear contraction are classified using a $F_{0}-B_{c}$ plane, where $F_{0}$ is the channel upstream Froude number and $B_{c}$ is the ratio of the exit $\left(W_{c}\right)$ and upstream channel width $\left(W_{0}\right)$, see Fig. 2.1. Although an approximate description of the flow characteristics, the reduced one-dimensional model allows for a rigorous prediction of the possible flow regimes. The majority of the predictions made via the one-dimensional asymptotic theory are further verified by solving the two-dimensional shallow granular equations using a discontinuous Galerkin finite element method (DGFEM). Solving the two-dimensional shallow granular equations through DGFEM not only helps verify the asymptotic theory but, more importantly, it scrutinises the applicability of the constitutive friction law in a two-dimensional setting.

\subsection{ASYMPTOTIC THEORY}

Rapid free-surface granular flows are considered as shallow when the ratio of the characteristic length- and velocity-scales in the normal to streamwise direction is small $(H / L \ll$ 1). Thereby researchers often depth-average the three-dimensional mass and momen- 
tum balance equations utilising asymptotic analysis [e.g. 11] together with a series of approximations [see e.g. 12, 13]. This results in the dimensional depth-averaged shallow granular equations

$$
\begin{gathered}
h_{t}+(h u)_{x}+(h v)_{y}=0 \\
(h u)_{t}+\left(h u^{2}+K \frac{h^{2}}{2} g_{n}\right)_{x}+(h u v)_{y}=g_{n} h\left(\tan \theta-\mu(h, \vec{u}) \frac{u}{|\vec{u}|}\right) \\
(h v)_{t}+(h u v)_{x}+\left(h v^{2}+K \frac{h^{2}}{2} g_{n}\right)_{y}=-g_{n} h \mu(h, \vec{u}) \frac{v}{|\vec{u}|}
\end{gathered}
$$

where the conservation of mass and momentum is represented in terms of the flow depth $h=h(x, y, t)$ and depth-averaged velocity $\vec{u}:=(u(x, y, t), v(x, y, t))$. Note that variables $x, y$ and $t$ represent the spatial and temporal coordinates with $g_{n}=g \cos \theta$ representing the acceleration due to gravity normal to the channel inclination $\theta$, see Fig. 2.1. Moreover, the variables $\mu(h, \vec{u})$ and $K$ denote the macroscopic basal friction coefficient and a material constant representing stress anisotropy. In addition to these variables, the subscripts $t, x$, and $y$ denote the respective partial derivatives.

Similar to the shallowness assumption utilised for depth-averaging, the ratio of the characteristic length- and velocity-scales in the normal direction to the cross-slope direction are also considered to be small $(H / W \ll 1)$. Thus depth-averaged flow quantities are further averaged across the channel as well, see Appendix 2.A. On width-averaging the shallow granular model (2.1) and further ignoring the higher-order Reynold's stress terms, the two-dimensional system of equations is reduced to a leading order one-dimensional (1D) depth- and width-averaged shallow granular model. For a constant basal topography, the $1 D$ model is

$$
\begin{aligned}
(h W)_{t}+(h u W)_{x} & =0 \\
(h W u)_{t}+\left(h W u^{2}\right)_{x}+\frac{1}{2} g_{n} K W\left(h^{2}\right)_{x} & =g_{n} h W(\tan \theta-\mu(h, u)) .
\end{aligned}
$$

Note that after width-averaging, the flow quantities $h$ and $u$ become independent of the $y$-coordinate, i.e. $h=h(x, t)$ and $u=u(x, t)$. Moreover, $W=W(x)$ defines the width of the channel. The channel has a constant width $W(x)=W_{0}$ for $x_{0} \leq x \leq x_{m}$ whereas for $x_{m} \leq x \leq x_{c}$ the channel's width linearly decreases from $W\left(x_{m}\right)=W_{0}$ to a minimum channel width at the channel's exit, i.e. $W\left(x_{c}\right)=W_{c}$. Note that the variables $x_{0}$ and $x_{m}$ denote the $x$-coordinate of the channel's and the contraction's entrance, respectively. Fig. 2.1 illustrates the schematic of the inclined channel with a linear contraction.

Given the above nomenclature, we introduce the following dimensionless variables denoted by primes,

$$
t=\frac{h_{l}}{u_{l}} t^{\prime}, \quad x=h_{l} x^{\prime}, \quad u=u_{l} u^{\prime}, \quad h=h_{l} h^{\prime}, \quad W=W_{l} W^{\prime}, \quad g_{n}=\frac{u_{l}^{2}}{h_{l}} g_{n}^{\prime},
$$

where $u_{l}, h_{l}$ and $W_{l}$ are typical values for the flow velocity, flow depth and channel width, respectively. Additionally, we also define the upstream Froude number as $F_{l}=$ 
$u_{l} / \sqrt{g_{n} h_{l}}=1 / \sqrt{g_{n}^{\prime}}$. Substituting the above scaled variables in (2.2) results in a nondimensional depth- and width-averaged shallow-layer model

$$
\begin{gathered}
(h W)_{t}+(h u W)_{x}=0 \\
u_{t}+u u_{x}+\frac{1}{F_{l}^{2}} h_{x}=\frac{1}{F_{l}^{2}}[\tan \theta-\mu(h, u)],
\end{gathered}
$$

with $1 / F_{l}^{2}$ playing the role of dimensionless gravity $g_{n}^{\prime}=1 / F_{l}^{2}$. For simplicity, we drop the primes and consider an isotropic flow scenario where $K=1$. Overall, the above one-dimensional shallow-layer model (2.4) consists of the continuity equation and the downslope momentum equation. However, in order to have a closed system of shallowlayer equations we need a constitutive friction law $\mu(h, u)$, which is described in the following section.

\subsubsection{Constitutive LaW/Closure Relation}

The basic difference between the shallow-layer fluid model and a granular one, i.e. (2.1), is the presence of a basal friction coefficient $\mu$ that is defined as the ratio of the shear to normal traction at the base. Some of the previously developed dry granular models incorporated a dry Coulomb-like friction law [10]. However, as stated by Pouliquen [36], the Coulomb-like constant friction law holds only in two cases:

1. When the inclined channel is smooth, fully developed uniform flows are found to exist at one critical inclination angle $\theta_{s}$ [37-39]. Above this angle the material accelerates and below this angle the flowing material eventually stops. The rheological properties of flows over smooth channels are well described by a constant friction constant, which equals the tangent of the angle of friction between the material and the base, i.e. $\mu=\tan \theta_{s}$.

2. Similarly, experimental studies also show that the constant friction coefficient holds for accelerating flows over rough channels at higher inclinations $[37,40]$. The experimental measurements of the shear forces at the bed show that the friction coefficient is independent of the downslope flow velocity.

However, for an intermediate range of angles where steady uniform flows reside [36, $41,42]$, the simple Coulomb friction law fails to describe the flow rheology on channels with rough beds. Using accurate experimental measurement techniques, Pouliquen [36] and Forterre and Pouliquen [43] empirically determined a scaling which allows to predict the variation in the mean (depth-averaged) velocity as a function of the channel inclination, flow depth and channel roughness,

$$
F=\frac{u}{\sqrt{g h}}=\beta \frac{h}{h_{\text {stop }}(\theta)}+\gamma,
$$

where the variable $h_{\text {stop }}(\theta)$ denotes the critical thickness where the flow arrests or comes to a halt with $\beta$ and $\gamma$ as constants. More importantly, the variable $h_{\text {stop }}(\theta)$ in (2.5) captures the effects of the channel roughness, channel inclination and other features like particle size without any experimental velocity measurements. Thereby, implying that 
each channel inclination has a unique critical thickness $h_{\text {stop }}(\theta)$, which depends on the channel roughness and particle size. For more details concerning the measurement of $h_{\text {stop }}(\theta)$, see Pouliquen [36]. Given this scaling law at hand, Pouliquen and Forterre [24] further expressed the crictical flow height as a function of the channel's angle of inclination,

$$
\frac{h_{\text {stop }}(\theta)}{A d}=\frac{\tan \left(\delta_{2}\right)-\tan (\theta)}{\tan (\theta)-\tan \left(\delta_{1}\right)}, \delta_{1}<\theta<\delta_{2},
$$

with $d$ as the grain (particle) diameter and $A$ as a characteristic dimensionless length scale over which the friction varies. Additionally, the above empirical friction law (2.6) is characterised by two angles: $\delta_{1}$, below which the friction dominates over gravity and the material comes to rest, and $\delta_{2}$ above which the material accelerates as gravity dominates friction. It is between these two angles where steady flows reside. On combining (2.5) and (2.6) and assuming the steady state flow assumption $\mu=\tan \theta$ to hold (approximately) in the dynamic case as well, one obtains an improved empirical friction law, valid for lower values of the Froude number,

$$
\mu=\mu(h, F)=\tan \left(\delta_{1}\right)+\frac{\tan \left(\delta_{2}\right)-\tan \left(\delta_{1}\right)}{\beta h /(A d(F-\gamma))+1} .
$$

As $\delta_{1} \rightarrow \delta_{2}$, the Coulomb's model is recovered, see Grigorian et al. [9].

\subsubsection{STEADY STATE SOLUTIONS}

By utilising the above stated macro-scale constitutive friction law (2.7), steady flow states in the channel with a linearised contraction can be predicted using the shallow-layer granular model (2.4).

We begin by defining the Froude number in terms of the non-dimensional variables as

$$
F(x)=F_{l} \frac{u(x)}{\sqrt{h(x)}},
$$

where $F_{l}=u_{l} / \sqrt{g_{n} h_{l}}$ equals $F_{0}$ for values $u_{0}, W_{0}$ and $h_{0}$ at $x=x_{0}$, which is near the sluice gate located upstream of the channel, or $F_{l}$ equals $F_{m}$ for values $u_{m}, W_{m}=W_{0}$ and $h_{m}$ at the contraction entrance $x=x_{m}$, see Fig. 2.1. Note that, even after scaling (2.2) and the other variables, we still retain the parameters $F_{l}=F_{m}$ or $F_{0}, B_{c}=W_{c} / W_{0}$, the source term $\tan \theta-\mu(h, F)$ and the dimensionless downslope coordinates $x_{c}$ and $x=$ $x_{m}$ or $x_{0}$.

For steady flows, the continuity equation $(h u W)_{x}=0$ implies a constant volume flux $h u W=Q$ where $Q$ denotes the integration constant. Morover, for our scaling (2.3) the flux $Q=1$. Thereby, using (2.4), the steady state momentum balance equation in a conservative form is

$$
F_{l}^{2}\left(\frac{u^{2}}{2}\right)_{x}+h_{x}=\tan \theta-\mu(h, F) .
$$

Using $u^{2}=\frac{F^{2} h}{F_{l}^{2}}$, we obtain

$$
\frac{d}{d x}\left[\left(1+\frac{F^{2}}{2}\right) h\right]=\tan \theta-\mu(h, F)
$$




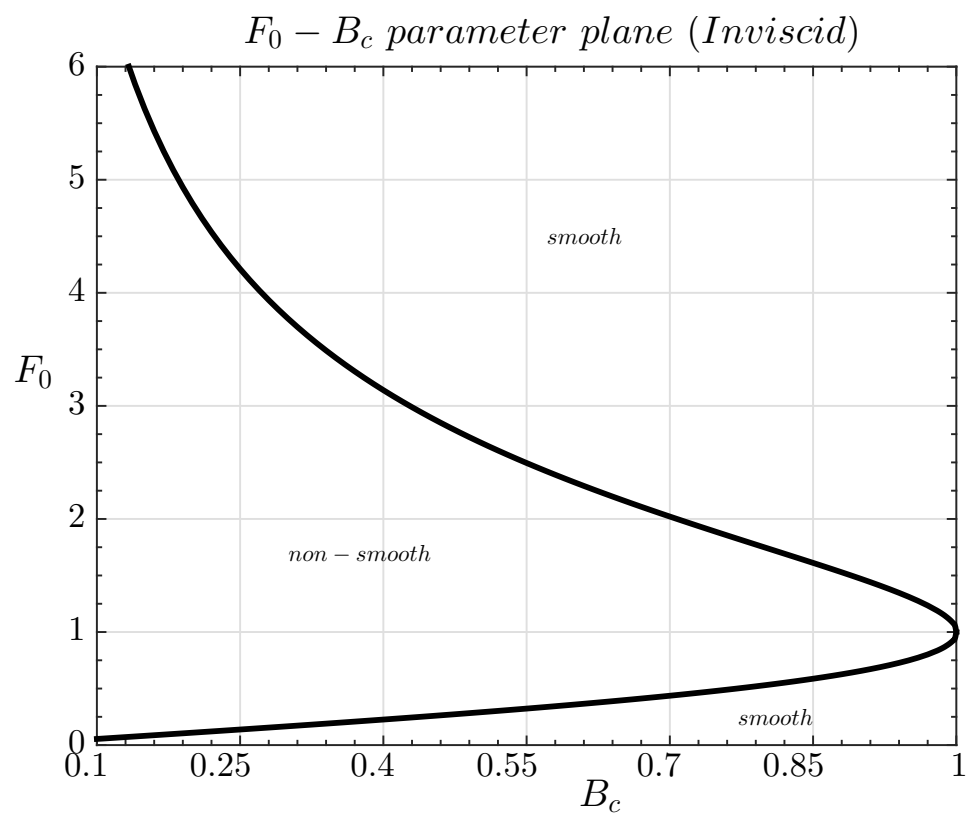

Figure 2.2: The solid curve divides the $F_{0}-B_{c}$ parameter plane into regions where either smooth or nonsmooth flow profiles exist for the inviscid scenario, i.e. when the friction term is approximately zero in (2.12).

and derive the expressions for the flow height $h(x)$ and its derivative, which are

$$
h=\left(\frac{Q F_{l}}{W F}\right)^{2 / 3} \text { and } \frac{d h}{d x}=-\frac{2}{3}\left[\frac{h}{F} F_{x}+\frac{h}{W} W_{x}\right] .
$$

On combining (2.10) and (2.11), we arrive at our simplified one-dimensional shallow granular model expressed in terms of the Froude number

$$
\frac{d F}{d x}=\underbrace{\frac{1}{2} \frac{\left(F^{2}+2\right) F}{\left(F^{2}-1\right)} \frac{1}{W} \frac{d W}{d x}}_{\text {Geometry }}+\underbrace{\frac{3}{2} \frac{[\tan \theta-\mu(h, F)]}{\left(Q F_{l}\right)^{2 / 3}} \frac{W^{2 / 3} F^{5 / 3}}{\left(F^{2}-1\right)}}_{\text {Friction }} .
$$

For details concerning the derivation of (2.11) and (2.12), see Appendix 2.A.3 and Appendix. 2.A.4. Additionally, (2.9) is analogous to equation (5) in Akers and Bokhove [44] when the source term $[\tan \theta-\mu(h, F)]$ is replaced with $-C_{d} F^{2}$ where $C_{d}$ represents the frictional drag in hydraulics. Thus resulting in the one-dimensional hydraulic theory.

\section{INVISCID FLOWS}

Given (2.12), we define a steady flow as inviscid when the friction term on the RHS of (2.12) is approximately zero, which typically occurs when $\mu \approx \tan \theta$. Thereby, (2.12) can be further simplified as

$$
\frac{d F}{d x}=\frac{1}{2}\left(\frac{F^{2}+2}{F^{2}-1}\right) \frac{F}{W} \frac{d W}{d x} .
$$


On a closer look, (2.13) can be analytically integrated with respect to $x$, see Appendix 2.A.5, from a point channel upstream say $x=x_{0}$ (channel entrance) or $x_{m}$ (contraction entrance) to some point $x$ downstream of the channel. As a result, the definite integral yields

$$
\frac{F_{l}}{F}\left(\frac{2+F^{2}}{2+F_{l}^{2}}\right)^{3 / 2}=\frac{W}{W_{0}},
$$

with $F_{l}=F_{0}$ when (2.13) is integrated from $x=x_{0}$ to some point downstream, whereas $F_{l}=F_{m}$ when (2.13) is integrated from $x=x_{m}$ to some point downstream. Besides $F_{l}$ taking the values $F_{0}$ or $F_{m}$, we further prescribe $F\left(x_{c}\right)=1$ at the channel exit, which is also known as the critical nozzle condition in the field of gas dynamics [45]. Moreover, this boundary condition at the channel exit implies that the flow at the channel's exit is 'sonic' or 'critical', signifying that the downslope flow velocity $u$ equals the gravitational wave speed $\sqrt{h} / F_{l}$, which in terms of dimensional quantities means that the downslope flow velocity $u$ equals $\sqrt{g_{n} h}$. As a result, the analytical inviscid solution to (2.13) together with the boundary conditions $F\left(x_{c}\right)=1$ and $F_{l}=F_{0}$ at $x=x_{0}$, is

$$
F_{0}\left(\frac{3}{2+F_{0}^{2}}\right)^{3 / 2}=B_{c}
$$

where $B_{c}:=W_{c} / W_{0}$.

Fig. 2.2 illustrates the corresponding analytical solution to (2.15) on a $F_{0}-B_{c}$ parameter plane that is divided into regions classified as smooth and non-smooth flow regions. For example, when a pair of $\left(F_{0}, B_{c}\right)$ is chosen from a region labelled smooth, the corresponding Froude $F(x)$ and height $h(x)$ profile would be smooth without any discontinuities in the flow profile. Similarly, when a pair of $\left(F_{0}, B_{c}\right)$ is chosen from the region labelled non-smooth, the resulting flow would contain a flow with discontinuities or an upstream moving shock. Note that flows are defined as subcritical when $F<1$ and supercritical when $F>1$.

\section{VISCID (FRICTIONAL) FLOWS}

Similar to the solid curve in Fig. 2.2, to obtain the demarcating curves for granular flows with frictional effects, we again integrate the ordinary differential equation (ODE) (2.12). However, we integrate from the channel's exit $x=x_{c}$ to a point upstream till the contraction's entrance at $x=x_{m}$ or till channel's entrance at $x=x_{0}$. As no analytical solution exists for this case, we numerically integrate (2.12) utilising a fourth-order Runge-Kutta scheme together with the earlier mentioned Houghton and Kasahara [45] critical exit condition $F\left(x_{c}\right)=1$, channel opening ratio $B_{c}=W_{c} / W_{0}$ and the width $W=W(x)$. Additionally, the Froude number $F_{l}$ and depth $h_{l}$ are prescribed upstream of the channel at $x=x_{l}$ with $F_{l}=F_{0}$ at the channel's entrance or $F_{l}=F_{m}$ at the contraction's entrance. However, before we numerically solve the ODE (2.12), we rearrange it as

$$
\underbrace{\frac{2}{3}\left(\frac{F^{2}-1}{F^{2}}\right) \frac{d F}{d x}}_{\text {LHS }}=\underbrace{\left(\frac{F^{2}+2}{3 F}\right) \frac{1}{W} \frac{d W}{d x}+\frac{F^{-1 / 3} W^{2 / 3}}{\left(Q F_{l}\right)^{2 / 3}}(\tan \theta-\mu(F))}_{\text {RHS }},
$$

to address an argument explained as follows. 


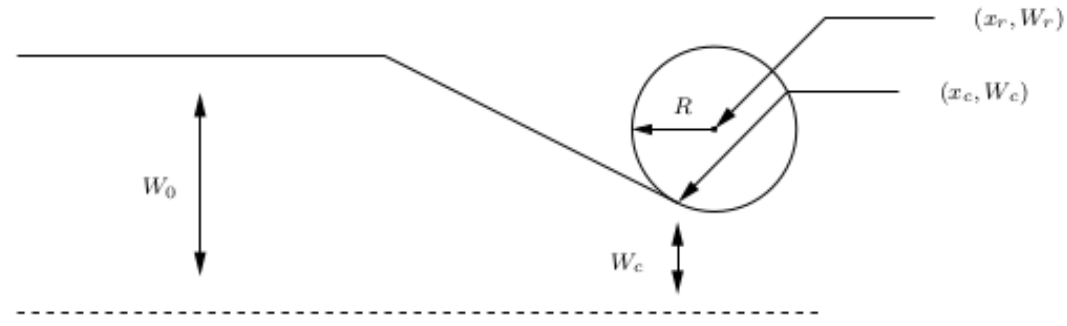

Figure 2.3: Illustration of the modified sidewall geometry, where a circle is fitted at the contraction exit $x_{c}$. As $R \rightarrow 0$, we arrive at the initial channel geometry at the contraction exit.

From a strict mathematical point of view, the left hand side of the above rearranged equation (2.16) can become zero at a certain $x$-location (point) either when $d F / d x=0$ or when $F=1$. Thereby, for (2.16) to be valid, the RHS also has to be zero at this particular point. Note that the reverse case also holds, i.e. if the RHS is zero at some point then the LHS has to be zero at the same point for (2.16) to hold. However, for the critical case where $F\left(x_{c}\right)=1$, (2.16) does not hold at $x=x_{c}$ because the linear contraction function $W(x)$ does not allow the RHS to be zero at the contraction exit. Thereby, in order to obtain the solid curves similar to the ones illustrated in Fig. 2.2, demarcating lines for frictional flows are obtained by integrating the ODE (2.12) in the following two ways:

(i) Non-regularised approach:

In this approach, the ODE (2.12) is integrated starting from the initial contraction exit, $x_{c}$, with Froude number $F\left(x_{c}\right)=\lim _{\varepsilon \rightarrow 0} 1 \pm \varepsilon$ such that we can avoid the LHS $\neq$ RHS situation at the contraction exit and determine a finite value for $d F / d x$. Thereby, we either begin with $F\left(x_{c}\right)=\lim _{\varepsilon \rightarrow 0} 1+\varepsilon$ for supercritical flows or $F\left(x_{c}\right)=\lim _{\varepsilon \rightarrow 0} 1-\varepsilon$ for subcritical flows. In this work, we defined $\varepsilon=1 e^{-10}$. Note that this is also the same approach that was followed by Akers and Bokhove [44] in the work concerning hydraulic flows.

(ii) Regularised approach:

The other approach is by rounding the contraction exit with an infinitesimal circle, see Fig. 2.3, and then determining the finite slope $d F / d x$ such that LHS=RHS in (2.16). This is the regularised approach, where by fitting a circle at the channel exit $x_{c}$ we determine the slope $d W / d x$ in (2.16) such that the LHS=RHS=0 at some point on the circle, see Appendix 2.B for more details.

Choosing a circle is convenient, as it has an infinite number of slopes and can be smoothly fitted at the contraction exit $x=x_{c}$. Moreover, as the radius $R \rightarrow 0$, we return to the extended or regularised sidewall geometry with a new contraction exit, $x_{c n e w}$, where the RHS in (2.16) equals zero. However, once regularised, we then arrive at a classical 

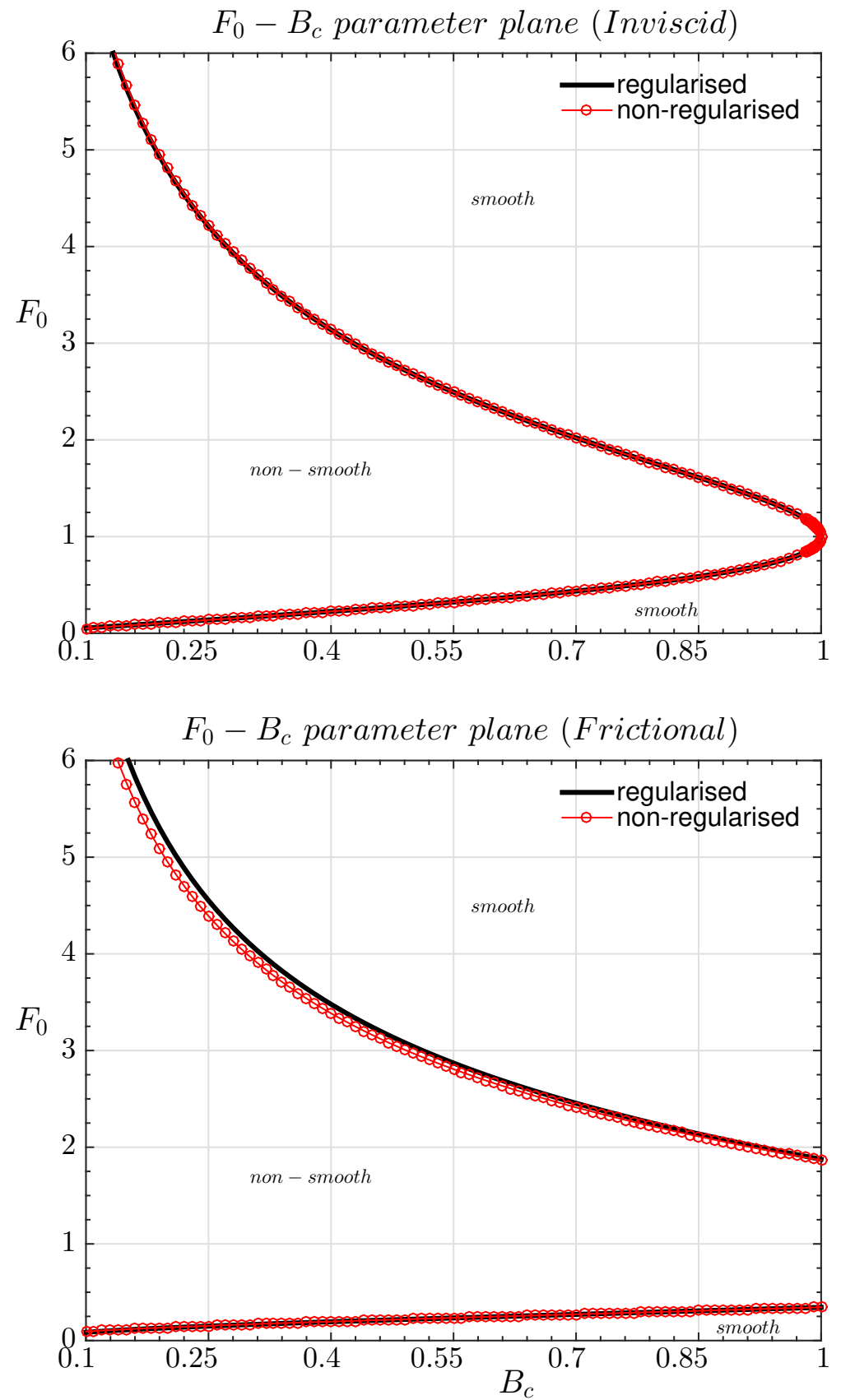

Figure 2.4: Non-regularised and regularised approaches are compared for inviscid (top) and frictional (bottom) flows where their respective numerical solutions to the governing ODE (2.12) divide the $F_{0}-B_{c}$ plane into regions corresponding to non-smooth and smooth flows. The solid demarcating lines correspond to the regularised approach whereas the red circles correspond to the non-regularised approach. Note that we considered a channel inclined at $\theta=30^{\circ}$ with the friction parameters $\delta_{1}=17.561, \delta_{2}=32.257, \beta=0.191, A=3.836$, $d=1$ and $\gamma=-0.045$; for more details see Table 1 in Weinhart et al. [25]. 
limit's problem

$$
\lim _{F\left(x_{\text {cnew }}\right) \rightarrow 1} \frac{2}{3}\left(\frac{F^{2}-1}{F^{2}}\right) \frac{d F}{d x}=\left(\frac{F^{2}+2}{3 F}\right) \frac{d(\ln W)}{d x}+\frac{F^{-1 / 3} W^{2 / 3}}{\left(Q F_{l}\right)^{2 / 3}}(\tan \theta-\mu(F)),
$$

which is undefined as both the numerator and denominator become zero. Thereby, equation (2.17) is solved by utilising the Taylor series expansion where the finite slope $d F / d x$ at the new contraction exit $x=x_{\text {cnew }}$ is determined for both the inviscid and viscous cases, see Appendix 2.B. Moreover, the Taylor series approach for regularisation is a novel extension to the non-regularised approach taken by Akers and Bokhove [44]. As a result, on regularisation we (i) rectify the mathematical inconsistency in the approach of Akers and Bokhove [44] and, more importantly, (ii) find that when the radius of the circle $R \rightarrow 0$, the newly obtained contraction exit location $x_{\text {cnew }} \rightarrow x_{c}$ such that LHS $=$ RHS is established. A regularised approach allows us to directly utilise the critical boundary condition $F\left(x_{\text {cnew }}\right)=1$ to integrate the ODE starting from the new channel exit $x=x_{\text {cnew }}$. Note that for regularisation, we considered an infinitesimal circle of radius $R=10^{-7}$ units.

To compare, both, regularised and non-regularised approaches, the ODE (2.12) is integrated to a point upstream, $x=x_{0}$, to find a new estimate for $F_{l}=F_{0}$. Since, the scaling parameter $F_{0}$ is unknown beforehand (as it is part of the solution), the correct value is found iteratively in both the approaches. As an educated guess, we take the initial value for $F_{l}=F_{0}\left(B_{c}\right)$ as the one obtained from the solution for the inviscid case, where $F_{0}$ is a function of $B_{c}$. Given this, we proceed iteratively until convergence is reached. As a result, Fig. 2.4 shows the demarcation curves obtained, for inviscid and frictional flows, using both the regularised and non-regularised approach. As illustrated, the resulting solutions are in excellent agreement for inviscid flows and subcritical frictional flows $(F<1)$. However, for supercritical frictional flows $(F>1)$, the non-regularised approach marginally under predicts the boundary demarcating the smooth and nonsmooth flows. Although regularisation marginally affects the end predictions in Fig. 2.4, it is still essential from a mathematical point of view.

\subsubsection{SHOCK SOLUTIONS}

The closed system of equations (2.4) is hyperbolic and thus the flows can develop discontinuities in finite time. By discontinuities, we imply shocks/jumps/bores in the height and velocity of the flow that either propagates upstream at a certain shock speed or remain steady. For example, let us consider an inclined channel with a particle reservoir located upstream and a sufficiently narrow exit-opening. Given this, we prescribe an upstream supercritical Froude number $(F>1)$ by slowly opening the reservoir gate such that the scaled flow height and velocity is unity, i.e. $h=u=1$, thereby, implying that the upstream Froude variable $F\left(x=x_{0}\right)=F_{0}$. Now as the flow encounters the region with contracting sidewalls with a sufficiently narrow contraction opening, a granular bore arises because the contraction region chokes the flow by hampering the flux of particles through the contraction. As a result, we observe a granular bore travelling upstream of the channel, in the direction opposite to the flow. Both in the inviscid and frictional flow scenarios, the granular bore/jump keeps propagating upstream since the flow is constant in the uniform channel region. However, for hydraulic flows, Akers [46] and 
Akers and Bokhove [44] predicted that, besides upstream moving shocks, there also exists a range of contraction openings $\left(B_{c}\right)$ and upstream prescribed Froude numbers $\left(F_{0}\right)$ for which the flows can exhibit, either, smooth supercritical flows or flows with moving/steady jumps. Thus, for this particular range of contraction openings and upstream prescribed Froude numbers, there exist multiple flow states. Thereby, this section focusses on investigating and predicting such a range for monodisperse granular flows as well. In other words, the outcomes of this section will predict the upper boundaries (dashed lines) of the region labelled multiple states in Fig. 2.5a (inviscid) and Fig. 2.5b (frictional).

For inviscid granular flows, i.e. when $\mu \approx \tan \theta$, the prediction of the multiple states region is exactly the same as in the case of hydraulic flows $[44,46]$. However, for purposes of clarity and ease, we thoroughly rederive the corresponding inviscid shock relations, which are essential for determining the multiple states region. To begin with, we consider the same flow scenario as was considered in Sec. 3.3 of Akers [46], which is the upstreammoving-shock scenario. As illustrated in Fig. 6 of Akers [46], we also consider a shock positioned at $x_{s}=x_{s}(t)$ with speed $s=d x_{s} / d t$. As a convention, the shock speed $s$ is positive when moving upstream of the channel. Given this, we denote the flow height and velocity just upstream (left side) of the shock at $x=x_{u}$ as $h_{u}$ and $u_{u}$ while the flow height and velocity just downstream (right side) of the shock at $x=x_{1}$ is denoted as $h_{1}$ and $u_{1}$. Additionally, the flow conditions at the channel-exit $\left(x=x_{c}\right)$ are represented by the height $h_{c}$ and velocity $u_{c}$. Instead of relating the upstream prescribed conditions at $x=x_{0}$ directly with the conditions at the channel-exit, $x=x_{c}$, we first couple the flow conditions at the left side (upstream) of the shock to the ones downstream (right side) of the shock and then relate it to the flow height $h_{c}$ and velocity $u_{c}$ at the channel-exit. As a result, the set of jump conditions relating the upstream and downstream shock variables $\left(h_{u}, u_{u}\right.$ and $\left.h_{1}, u_{1}\right)$ are simply the standard shock relations corresponding to the inviscid shallow layer equations [e.g. 47], as frictional terms do not contribute.

$$
\begin{aligned}
\left(u_{u}+s\right) h_{u} & =\left(u_{1}+s\right) h_{1}, \\
\left(u_{u}+s\right)^{2} & =\frac{h_{1}}{2 F_{l}^{2}}\left(1+\frac{h_{1}}{h_{u}}\right) .
\end{aligned}
$$

For details concerning the derivation of the above equations (2.18), see Appendix 2.C where we substitute $h^{+}=h_{u}, u^{+}=u_{u}$ and $h^{-}=h_{1}, u^{-}=u_{1}$. However, in order to relate the upstream shock conditions $\left(h_{u}\right.$ and $\left.u_{u}\right)$ to the ones at the channel exit, we have to relate the downstream shock variables $\left(h_{1}\right.$ and $\left.u_{1}\right)$ to the values at the exit. For this purpose, we utilise the Bernoulli equations, corresponding to the inviscid case, together with the continuity equations in the contracting-sidewalls region. Thus resulting in

$$
\begin{aligned}
\frac{1}{2} u_{1}^{2}+h_{1} / F_{l}^{2} & =\frac{1}{2} u_{c}^{2}+h_{c} / F_{l}^{2}, \\
u_{1} h_{1} W_{1} & =u_{c} h_{c} W_{c} .
\end{aligned}
$$


However, so far, we have four equations (2.18) for five unknowns, $h_{1}, h_{c}, u_{1}, u_{c}$ and $s$. Thereby, for the fifth equation, we utilise the critical nozzle condition of Houghton and Kasahara [45], which implies that the Froude number at the channel exit is unity, $F\left(x_{c}\right)=1$, hence, leading to the fifth equation

$$
u_{c}^{2}=h_{c} / F_{l}^{2} \text {. }
$$

As a result, we have a system of five equations (2.18) for the five unknowns with $h_{u}, u_{u}$, $W_{1}$ and $W_{c}$ as prescribed values. However, we further manipulate (2.18) by introducing the following variables

$$
F_{u}=u_{u} F_{l} / \sqrt{h_{u}}, \quad S=s F_{l} / \sqrt{h_{u}}, \quad H_{1}=h_{1} / h_{u} \quad \text { and } \quad B_{1}=W_{c} / W_{1} .
$$

Next, we substitute these scaled variables $(2.19)_{1-3}$ into (2.18b) and arrive at a transformed jump condition,

$$
\left(\frac{\sqrt{h_{u}}}{F_{l}}\left(F_{u}+S\right)\right)^{2}=\frac{h_{1}}{2 F_{l}^{2}}\left(1+H_{1}\right) \underset{h_{u} / F_{l}^{2}}{\stackrel{\text { Taking out }}{=}} \underbrace{\left(F_{u}+S\right)^{2}=\frac{1}{2} H_{1}\left(1+H_{1}\right)}_{\text {Transformed jump condition \# } 1},
$$

where the jump condition (2.18b) is now expressed in terms of the scaled variables. In order to effectively express the complete system of equations (2.18) in terms of the scaled variables (2.19), we further derive three intermediate relations. To arrive at our first relation, we substitute $(2.19)_{3}$ into (2.18a) and further manipulate it by incorporating the scaled variables $(2.19)_{1-2}$, resulting in

$$
\begin{gathered}
\left(u_{u}+s\right)=\left(u_{1}+s\right) \frac{h_{1}}{h_{u}} \stackrel{H_{1}=h_{1} / h_{u}}{\Longrightarrow}\left(u_{u}+s\right)=\left(u_{1}+s\right) H_{1}, \\
\left(u_{u}+s\right)=\left(u_{1}+s\right) H_{1} \underset{S=s F_{l} / \sqrt{h_{u}}}{\stackrel{F_{u}=u_{u} F_{l} / \sqrt{h_{u}}}{\longrightarrow}} \frac{\sqrt{h_{u}}}{F_{l}}\left(F_{u}+\left(1-H_{1}\right) S\right)=u_{1} H_{1}, \\
\Rightarrow \underbrace{u_{1}^{2}=\frac{h_{u}}{H_{1}^{2} F_{l}^{2}}\left(F_{u}+\left(1-H_{1}\right) S\right)^{2},}_{\text {Relation \# 1 }}
\end{gathered}
$$

Similarly, we derive the second intermediate relation by first rearranging the jump condition (2.18b) and then substituting (i) the scaled variables (2.19) and (ii) the transformed jump condition (2.20) into it. Thus leading to

$$
\left(u_{u}+s\right)^{2}=\frac{h_{1}}{2 F_{l}^{2}}\left(1+\frac{h_{1}}{h_{u}}\right) \stackrel{(2.19)}{\Longrightarrow} h_{1}=2 h_{u} \frac{\left(F_{u}+S\right)^{2}}{1+H_{1}} \stackrel{(2.20)}{\Longrightarrow} \underbrace{h_{1}=h_{u} H_{1}}_{\text {Relation \#2 }} .
$$

Finally, the third intermediate relation is derived by substituting the scaled channel-exit $(2.19)_{4}$ and the critical condition (2.18e) into the mass continuity equation (2.18d), which then results in

$$
u_{1} h_{1} W_{1}=u_{c} h_{c} W_{c} \underset{B_{1}=W_{c} / W_{1}}{\stackrel{(2.19)_{4}}{\longrightarrow}} u_{1} h_{1}=u_{c} h_{c} B_{1} \underset{u_{c}^{2}=h_{c} / F_{l}^{2}}{\stackrel{(2.18 e)}{u_{c}^{2}=\left(\frac{u_{1} h_{1}}{B_{1} F_{l}^{2}}\right)^{2 / 3}}} .
$$


Given the above three intermediate relations (2.21)-(2.23) and the modified jump condition (2.20), we systematically arrive at the complete set of transformed and reduced shock relations as follows. We begin by substituting the critical condition (2.18e) and the relation (2.23) into the Bernoulli equation (2.18c), which is further manipulated by substituting the other two relations (2.21) and (2.22), resulting in

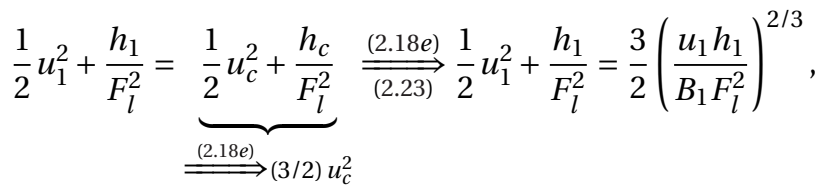

$$
\begin{aligned}
& \underset{(2.22)}{\stackrel{(2.21)}{\longrightarrow}} \frac{h_{u}}{2 H_{1}^{2} F_{l}^{2}}\left(F_{u}+\left(1-H_{1}\right) S\right)^{2}+\frac{h_{u}}{F_{l}^{2}} H_{1}=\frac{3}{2}\left(\frac{h_{u}^{3 / 2}}{F_{l}^{3}} \frac{\left(F_{u}+\left(1-H_{1}\right) S\right)}{B_{1}}\right)^{2 / 3} \text {, } \\
& \underset{h_{u} / F_{l}^{2}}{\stackrel{\text { Factoring out }}{=}} \underbrace{\frac{1}{2}\left[F_{u}+\left(1-H_{1}\right) S\right]^{2}=\frac{3}{2} H_{1}^{2}\left[\frac{F_{u}+\left(1-H_{1}\right) S}{B_{1}}\right]^{2 / 3}-H_{1}^{3}}_{\text {Transformed jurp }} .
\end{aligned}
$$

Hence, through the above transformations and intermediate relations (2.20)-(2.24), the system of equations (2.18) is transformed and reduced in terms of the scaled variables (2.19) as

$$
\begin{gathered}
\left(F_{u}+S\right)^{2}=\frac{1}{2} H_{1}\left(1+H_{1}\right), \\
\frac{1}{2}\left[F_{u}+\left(1-H_{1}\right) S\right]^{2}=\frac{3}{2} H_{1}^{2}\left[\frac{F_{u}+\left(1-H_{1}\right) S}{B_{1}}\right]^{2 / 3}-H_{1}^{3} .
\end{gathered}
$$

On a closer look at the transformed shock relations (2.25), there exist two limits where either the height ratio $H_{1}=1$ or the shock speed $S=0$. For smooth flows, i.e. when $H_{1}=1$, the transformed shock relations (2.25) reduce to

$$
B_{1}=F_{u}\left(\frac{3}{2+F_{u}^{2}}\right)^{3 / 2},
$$

which is fully equivalent to the inviscid relation (2.15) when $F_{u}=F_{0}$ and $B_{1}=B_{c}$. Note that relation (2.15) is valid for both super- and subcritical flows and was utilised for predicting the solid line illustrated in Fig. 2.2. The other limit is the jump scenario where the shock speed $S=0$ and $H_{1} \neq 1$. However, before we proceed further, let us revisit the flowwith-a-jump scenario. As mentioned earlier, a granular bore emerges and travels in the upstream direction when an upstream prescribed supercritical flow $\left(F_{0}>1\right)$ encounters a sufficiently narrow contraction. By slightly increasing or decreasing the contraction width, the flow can also encounter a situation where the generated granular bore halts precisely at the entrance of the contraction $x=x_{m}$, such that the shock speed is zero, $S=0$. It is only when this threshold $(S=0)$ is surpassed by the flow, the generated bore begins to travel upstream at a finite shock speed $S>0$.

\section{STATIONARY SHOCKS IN INVISCID FLOWS}

In the following, we will determine the range of upstream prescribed supercritical Froude numbers $\left(F_{0}>1\right)$ and contraction openings $\left(B_{c}\right)$, which correspond to the case where 
granular bores have zero shock speed at the contraction entrance. To do so, a closer look at the flow with a jump/bore reveals that the flow is supercritical $F_{0}>1$ (just) upstream of this stationary bore and subcritical $F_{1}<1$ (just) downstream of the bore at the contraction entrance, whereafter it remains subcritical till the channel-exit where $F\left(x_{c}\right)=1$. In order to determine the range of upstream prescribed Froude numbers $\left(F_{0}>1\right)$ and the contraction openings $\left(B_{c}\right)$ corresponding to zero shock speed at the contraction entrance, i.e. the dashed line in Fig. 2.5a, we begin by assuming that we know $F_{1}$ (just) downstream of the shock. Thus, we can utilise the inviscid relation (2.15) with $F_{0}$ replaced with $F_{1}$ to result in

$$
B_{c}=F_{1}\left(\frac{3}{2+F_{1}^{2}}\right)^{3 / 2} \text {. }
$$

With the relation (2.27) for $F_{1}<1$, we now determine the upstream supercritical Froude numbers $\left(F_{0}>1\right)$ not only in terms of $F_{1}<1$ but also for which the shock speed $s=0$. To do so, we consider $h_{u}=h_{0}=1$ and $u_{u}=u_{0}=1$ such that $F_{u}=F_{0}$ and, thereby, further simplify the shock relation (2.25) $)_{1}$ to a quadratic equation $h_{1}^{2}-h_{1}-2 F_{0}^{2}=0$, whose physically valid root is $h_{1}=(1 / 2)\left(-1+\sqrt{1+8 F_{0}^{2}}\right)$. Note that $h_{1}$ is the flow height just downstream of the granular bore. In order to express the upstream Froude number in terms of $F_{1}$, we utilise the continuity condition $h_{1} u_{1}=h_{0} u_{0}$, which when rearranged results in $u_{0} / u_{1}=h_{1} / h_{0}$. Using (2.8), this is restated as $F_{0} / F_{1}=\left(h_{1} / h_{0}\right)^{3 / 2}$, which when substituted into $(2.25)_{1}$, results in another quadratic equation $\left(h_{0} / h_{1}\right)^{2}-\left(h_{0} / h_{1}\right)-2 F_{1}^{2}=0$, whose physically valid solution is $h_{0} / h_{1}=(1 / 2)\left(-1+\sqrt{1+8 F_{1}^{2}}\right)$. On further substitution of $F_{0} / F_{1}=\left(h_{1} / h_{0}\right)^{3 / 2}$, the solution to the quadratic equation is simplified to

$$
F_{0}=\sqrt{8} F_{1}\left[-1+\sqrt{\left(1+8 F_{1}^{2}\right)}\right]^{-3 / 2}>1 .
$$

Thus, for a known Froude number $F_{1}<1$, just downstream of the shock, expression (2.28) determines the upstream supercritical Froude number $\left(F_{0}>1\right)$ for which inviscid flows will develop bores with zero shock speed $(S=0)$ at the entrance of the contraction region $\left(x=x_{m}\right)$. More importantly, for a given range of contraction openings $B_{c}$, (just) downstream shock condition $F_{1}<1$ and the boundary condition $F\left(x_{c}\right)=1$, relation (2.28) now enables us to determine the range of upstream supercritical Froude numbers $F_{0}>1$ that will develop jumps/bores with zero shock speed $(S=0)$ at the entrance of the contraction. Thus leading to the dashed line in Fig. 2.5a. As a result, the solid and dashed line divides the $F_{0}-B_{c}$ plane in Fig. 2.5a, such that there exists a mixed region where both smooth flows and flows with moving shocks can exist. Hence, defining the multiple states region for inviscid flows.

\section{STATIONARY SHOCKS IN VISCID (FRICTIONAL) FLOWS}

On the other hand, besides upstream moving shocks or shocks with zero speed at the entrance of the contraction, there also exists the possibility of steady shocks in the contraction region. For hydraulic flows, [46] and [44] show that flows with moving jumps/bores become steady in the presence of hydraulic drag, $C_{d}$. Thereby, in order to determine a similar threshold for frictional granular flows, i.e. the dashed line in Fig. 2.5b, the 
(a)

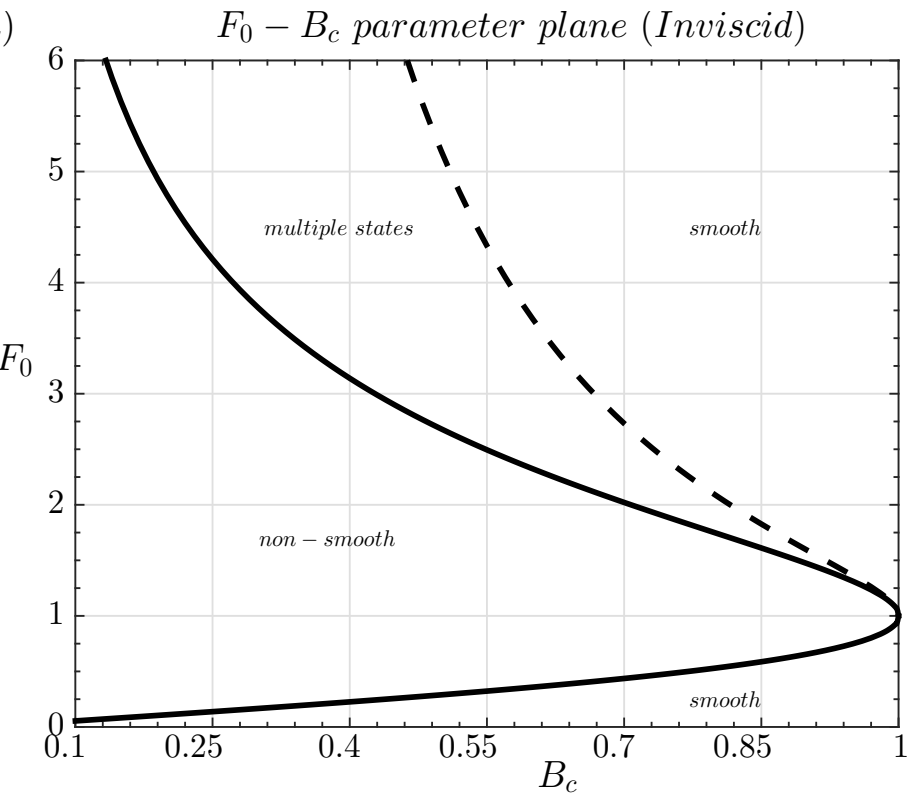

(b)

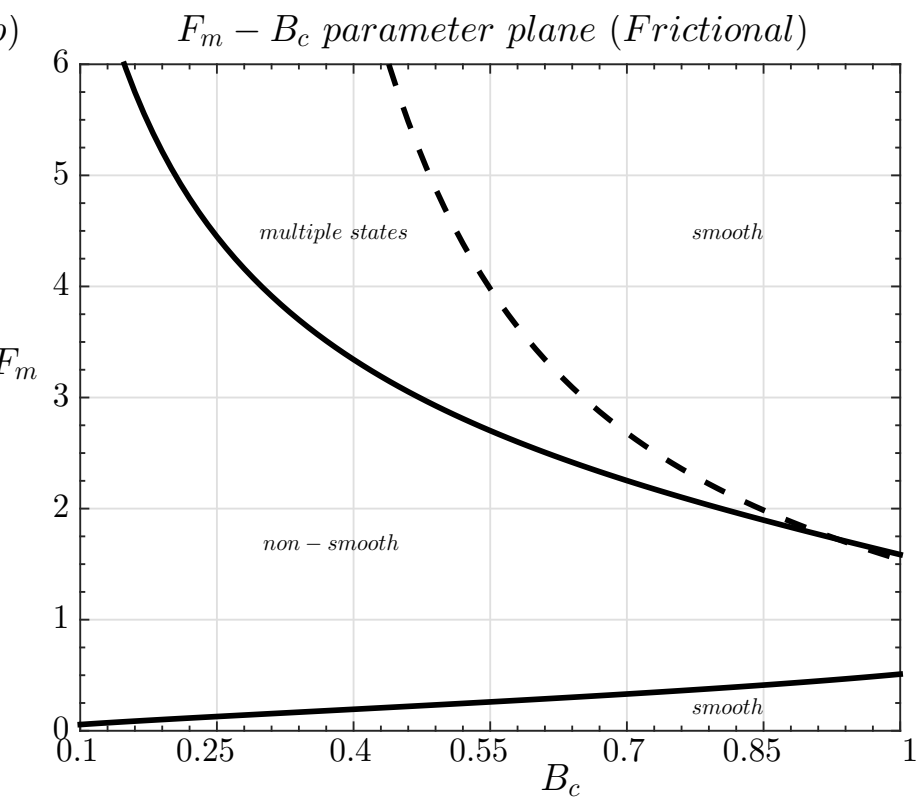

Figure 2.5: Illustrates parameter planes divided into regions of different flow regimes for both (a) inviscid and (b) frictional flows. Variables $F_{0}$ and $F_{m}$ correspond to the Froude value at the upstream channel and contraction entrance contraction whereas $B_{c}$ is the normalised channel opening width, respectively. Regions labelled as smooth contain pairs of $\left(F_{0}, B_{c}\right)$ in (a) and $\left(F_{m}, B_{c}\right)$ in (b), for which averaged smooth Froude and height profiles exist, i.e. profiles of $F$ vs. $x$ and $h$ vs. $x$ are either monotonically decreasing or increasing. Similarly, the non-smooth (discontinuous) region contains pairs corresponding to upstream moving or steady shocks. However, the multiple states region comprises of pairs demonstrating both smooth and non-smooth flow regimes. For inviscid flows, the $\left(F_{0}, B_{c}\right)$ pairs in the multiple states region of plot (a) exhibit flows that are either smooth or with upstream moving shocks. Similarly, for frictional flows, the $\left(F_{m}, B_{c}\right)$ pairs in the multiple states region of plot (b) illustrate flows that are either smooth or with steady shocks, which is in contrast to the 'upstream moving shocks' prediction made for inviscid flows. For more details concerning the frictional flow Froude and height profiles, see Fig. 2.6. 
(i)
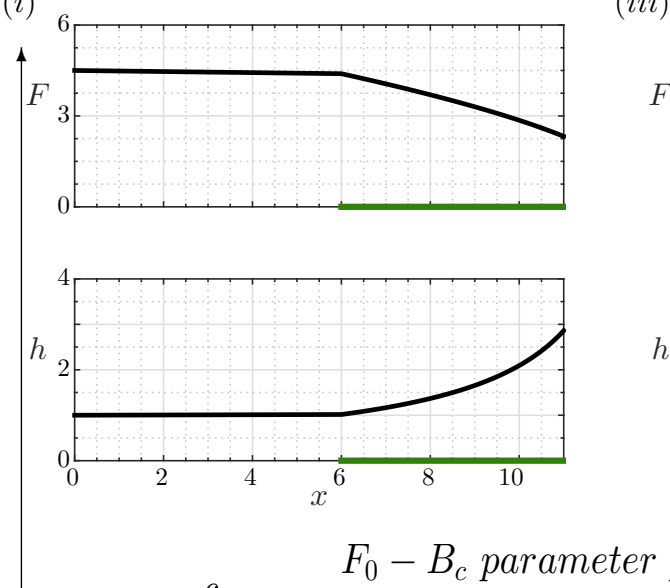

(iii)

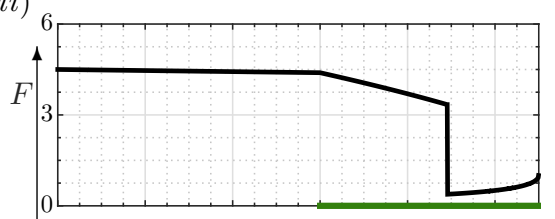


Bernoulli equations for the inviscid case are replaced by (2.12) from the shock's $x$ position to the contraction exit $\left(x_{c}\right)$. Similar to the inviscid case, we calculate the frictional shock conditions at the contraction entrance by integrating (2.12) from the channel exit to the contraction entrance with the critical channel exit condition $F\left(x_{c}\right)=1$. Given that, we denote the Froude number just downstream and upstream of the shock at the contraction entrance as $F_{1}$ and $F_{u}=F_{m}$, using the shock relation (2.25) 1 with $s=0, h_{u}=h_{m}$, $u_{u}=u_{m}$ and $F_{u}=F_{m}$, we arrive at the quadratic equation $\left(h_{1} / h_{m}\right)^{2}-\left(h_{1} / h_{m}\right)-2 F_{m}^{2}=0$, whose solution is

$$
\frac{h_{1}}{h_{m}}=\frac{1}{2}\left[-1+\sqrt{1+8 F_{m}^{2}}\right] .
$$

Similar to (2.28), (2.29) is restated in the same manner where the Froude number just upstream of the shock $\left(F_{m}\right)$ is represented in terms of $F_{1}$, which is the Froude number just downstream of the shock. Thereby resulting in

$$
F_{m}=\sqrt{8} F_{1}\left[-1+\sqrt{\left(1+8 F_{1}^{2}\right)}\right]^{-3 / 2}>1 .
$$

Note that the flow is supercritical $F_{m}>1$ (just) upstream of the shock and subcritical $F_{1}<1$ (just) downstream of the shock at the contraction entrance $x=x_{m}$.

Given $F_{m}$, the ODE (2.12) can be further integrated from $F=F_{m}>1$ at $x=x_{m}$ to find our next estimate of $F_{l}^{*}$ at the channel upstream, $x=x_{0}$. Generally, $F_{l}^{*} \neq F_{l}$, where $F_{l}$ is the scaling used in (2.12). One begins with the inviscid result $F_{l}=F_{0}\left(B_{c}\right)$ to determine the threshold also for frictional flows, which together with the solid lines acts as the demarcating curve for both $F_{m}-B_{c}$ and $F_{0}-B_{c}$ planes, see Fig. 2.5. Note that we utilise the regularised approach to determine these demarcating curves, as this approach is necessary to make the numerical analysis mathematically sound.

For frictional flows, the supercritical $(F>1)$ and subcritical $(F<1)$ flow profiles are illustrated via $F$ and $h$ versus $x$ plots in Fig. 2.6. These profiles are obtained by integrating from a point upstream of the channel into the downstream direction to the contraction exit. For flows with granular jumps (discontinuities in flow quantities), the critical condition at the channel exit is $F\left(x_{c}\right)=1$. Given this, we integrate from the channel exit and move into the upstream direction. The jumps in the flow quantities are computed by applying the jump conditions, and then finding a point where the downstream and upstream profiles match. Thereby, the flow profiles are efficiently computed from the novel one-dimensional granular hydraulic theory. However, in contrast to the predictions made for hydraulic flows [44], the multiple states region for frictional granular flows comprises of two flow states alone, Fig. 2.6(i) and Fig. 2.6(iii). These are the smooth supercritical flows and flows with steady jumps in the contraction region (reservoir state).

\subsection{VERIFICATION OF THE ONE-DIMENSIONAL THEORY VIA TWO- DIMENSIONAL NUMERICAL SOLUTIONS}

The asymptotic theory, presented in the Sec. 2.2, helps us to approximately predict and classify the flow regimes using a $F_{0}-B_{c}$ parameter plane. In order to verify the predictions made via the one-dimensional theory, they are compared with two-dimensional numerical solutions of the shallow granular equations (2.1). The additional degree of 
freedom helps us illustrate and interpret the results from the one-dimensional analysis. This way, the two-dimensional numerical solutions allow us to validate the onedimensional model with the constitutive friction law in a two-dimensional setting. Note that we use the same closure form (2.7) in one- and two-dimensional space.

\subsubsection{TWO-DIMENSIONAL DGFEM SOLUTIONS}

Two-dimensional (2D) numerical solutions are obtained by solving the shallow granular equations (2.1), along with the closure relation (2.7) for $\mu(h, F)$, using a discontinuous Galerkin finite element method (DGFEM). The method is a blend of both high resolution finite element and finite volume methods, which is often utilised for solving systems of partial differential equations, see for example Di Pietro and Ern [48]. For solving the system of shallow granular equations (2.1), we adopt the second order space-discontinuous Galerkin method of Tassi et al. [49] combined with the HLL numerical flux [50].

\subsubsection{TWO-DIMENSIONAL DGFEM VS. ONE-DIMENSIONAL ASYMPTOTIC THEORY}

For supercritical flows $(F>1)$, similar to the channel schematic in Fig. 2.1, we initially consider a sufficiently long and wide inclined channel with contracting sidewalls such that all the spatial coordinates $(x, y) \in[0,55] \times[0,5]$. The contracting section of the channel spans from $x=x_{m}=30$ to $x=x_{c}=55$ and ends with a channel exit ratio $B_{c}=0.4$, see Fig. 2.7(a). The channel configuration is chosen such that the ratios of the characteristic length-scales $H / L$ and $H / W$ are sufficiently small $(<<1)$ for the one-dimensional shallow granular theory to be valid [e.g. 10]. Hence, the length-scale ratios for the considered channel configuration, in Fig. 2.7(a), are $H / L=1 / 55$ and $H / W=1 / 5$.

To obtain the DGFEM two-dimensional solutions, the channel domain is discretised with a uniform quadrilateral mesh and is prescribed with suitable boundary conditions. At the channel's inflow boundary, we prescribe the flow height $h=1$ and a downstream velocity such that the inflow Froude number is $F_{0}=4.5$, whereas at the outflow boundary a free-flow condition is prescribed [53]. On the other hand, the channel sidewalls are prescribed with a solid wall boundary condition, as proposed in Ambati and Bokhove [54]. Note that the choice of prescribing $\left(F_{0}, B_{c}\right)=(4.5,0.4)$ corresponds to the $\left(F_{0}, B_{c}\right)$ pair $(4.5,0.4)$ in the multiple states region of Fig. 2.5 . The pair is marked by the '*' symbol.

On solving the dimensionless $2 \mathrm{D}$ system of shallow granular equations for the above prescribed boundary conditions, the resulting flow height profile illustrates weak oblique jumps in the deflecting sidewalls region of the channel, see Fig. 2.7(a) top panel. However, for our verification purpose, the two-dimensional depth-averaged flow profiles are further averaged in the $y$-direction (cross-slope) and compared with the solution profile predicted using the novel one-dimensional granular hydraulic theory. As a result, the bottom panel of Fig. 2.7(a) plots, both, the averaged two- (red circles) and onedimensional (solid line) solutions. One can conclude that the one-dimensional asymptotic theory is in excellent agreement with the two-dimensional DGFEM solution for a supercritical flow.

On the other hand, a recent experimental study, which focussed on the channel's sidewall effects [55], reported an almost uniform free-surface velocity across a uniform channel when the characteristic length-scale ratio $H / W=1$. However, Jop et al. [55] 


\section{Supercritical flows:}

(a) Sufficiently long and wide channel with characteristic length-scales: $\frac{H}{L}=\frac{1}{55}$ and $\frac{H}{W}=\frac{1}{5}$
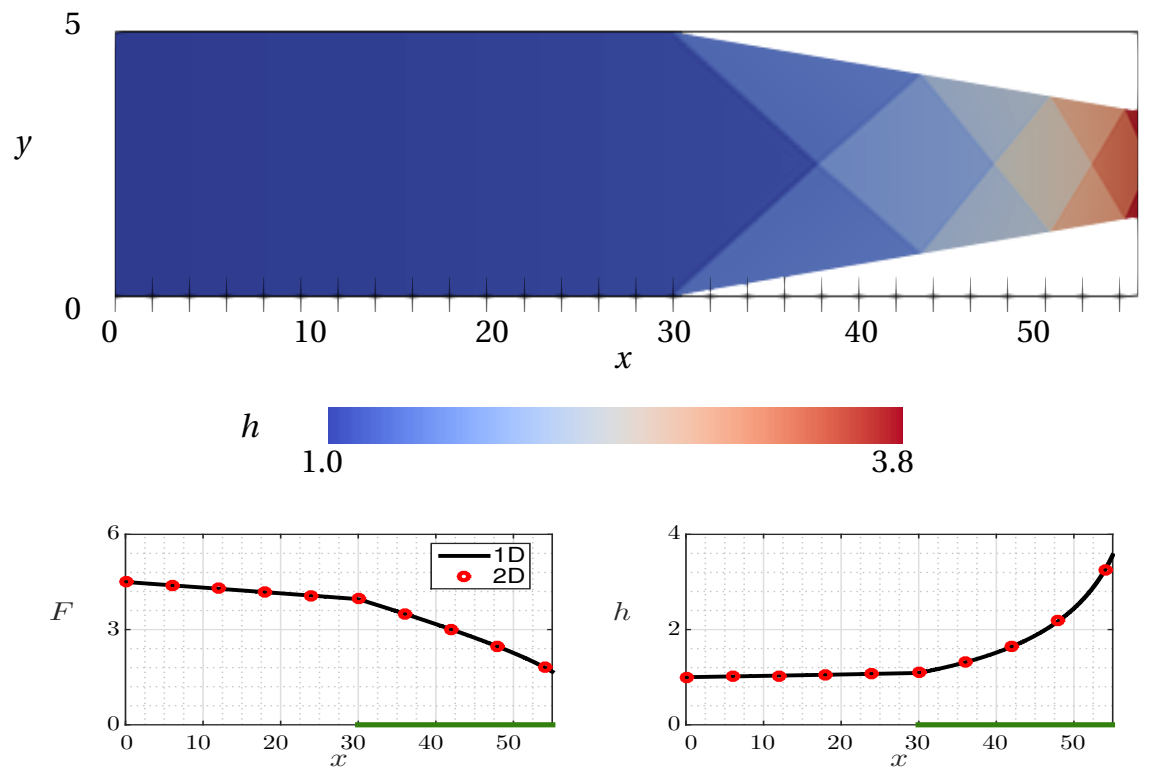

(b) Sufficiently still long but a narrow channel with $\frac{H}{L}=\frac{1}{11}$ and $\frac{H}{W}=1$
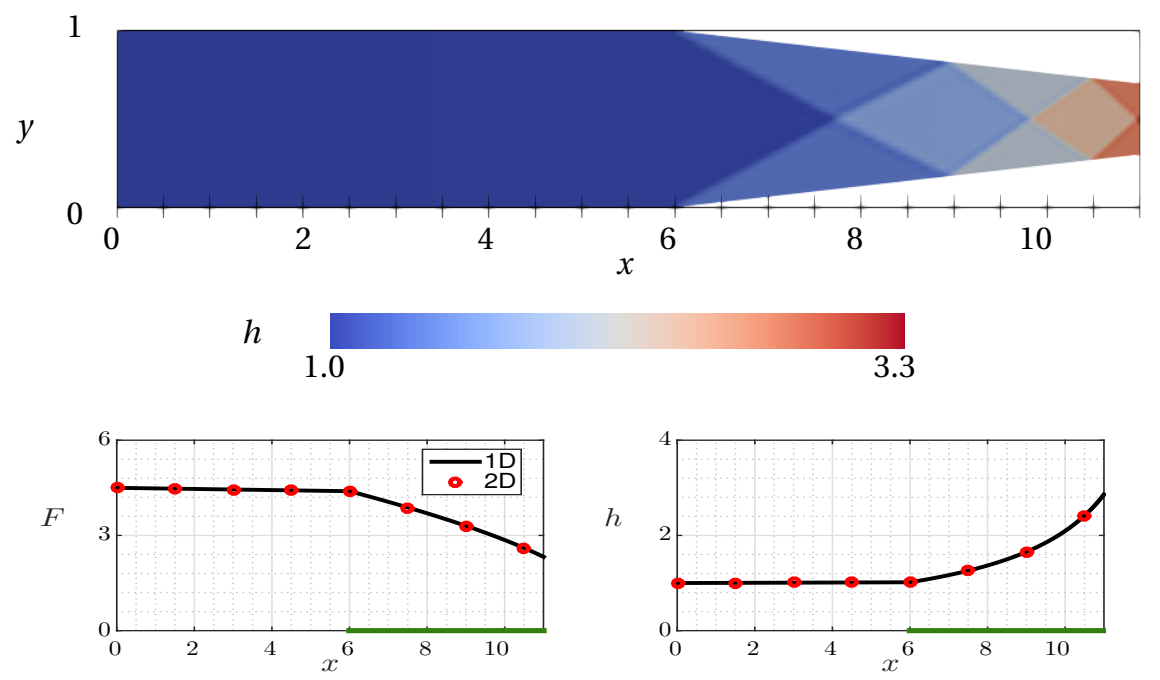

Figure 2.7: The top panels of (a) and (b) are colour plots of the flow height $h=h(x, y)$ as a function of downstream and cross-slope coordinates $x$ and $y$ for a prescribed upstream inflow Froude number for supercritical flow, $\left(F_{0}, B_{c}\right)=(4.5,0.4)$. The solutions are obtained by solving the two-dimensional shallow granular model using the discontinuous Galerkin finite element method software package hpGEM [51, 52]. On the other hand, bottom panels of (a) and (b) illustrate the corresponding Froude number $F=F(x)$ and flow height $h=h(x)$ profiles as a function of downstream coordinate $x$, respectively. The red circles denote the two-dimensional width-averaged DGFEM solutions whereas the solid lines represent the solutions obtained using our onedimensional theory. 
Subcritical flow: $\frac{H}{L}=\frac{1}{11}$ and $\frac{H}{W}=1$
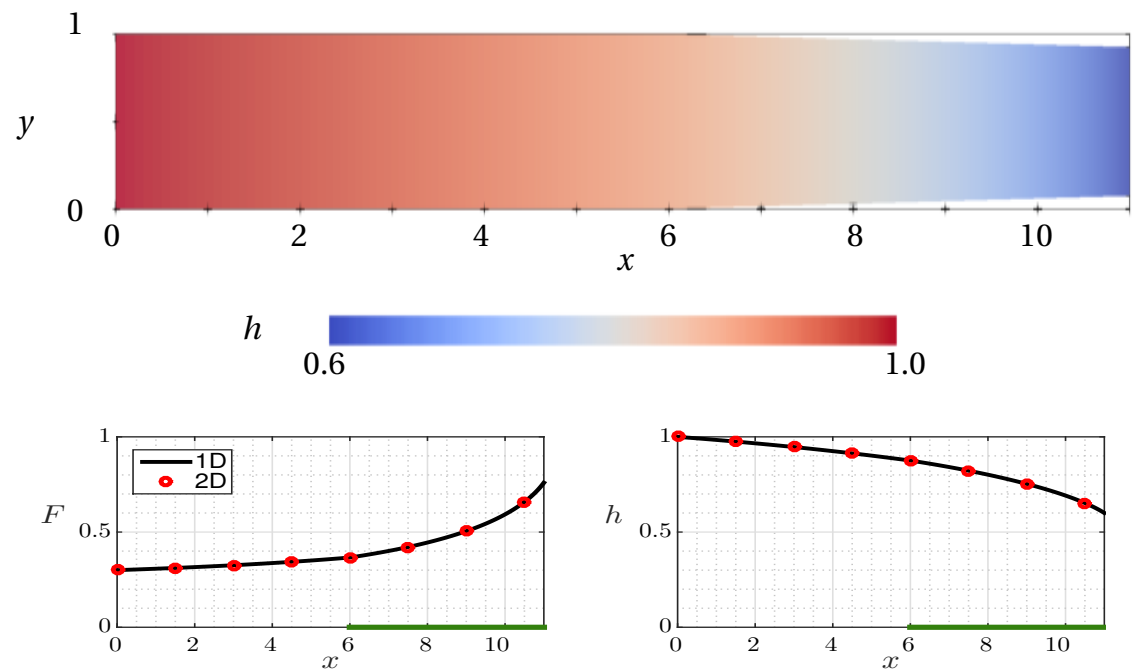

Figure 2.8: Similar to Fig. 2.7, the top panel illustrates the flow height $h=h(x, y)$ as a function of downstream and cross-slope coordinates $x$ and $y$ for a prescribed upstream inflow Froude number corresponding to a subcritical flow, $\left(F_{0}, B_{c}\right)=(0.3,0.85)$. On the other hand, the bottom panel illustrates the Froude number $F=F(x)$ and flow height $h=h(x)$ profiles as a function of downstream coordinate $x$. The red circles denote the two-dimensional width-averaged DGFEM solution and the solid lines represent the solution obtained using our one-dimensional theory. 
also report that as this uniform channel's width increases such that $H / W<<1$ the freesurface velocity becomes non-uniform especially at the channel sidewalls. As a result, given our theory does not include these experimentally observed sidewall effects, we consider a channel that is still sufficiently long but narrow such that $H / L=1 / 11$ and $H=W$ instead of $H / W=1 / 5(<<1)$, see Fig. 2.7(b). In this new channel configuration all the spatial coordinates $(x, y) \in[0,11] \times[0,1]$. More importantly, the contracting section spans from $x=x_{m}=6$ to $x=x_{c}=11$ such that (i) the channel exit ratio is still $B_{c}=0.4$ and (ii) the angle made by the contracting sidewall with the horizontal is still preserved. In other words, the angle made by the contracting sidewall with the horizontal is the same in the contracting channels considered in Fig. 2.7(a) and Fig. 2.7(b). Note that the $F_{0}-B_{c}$ phaseplots, Fig. 2.5, correspond to the channel configuration where $(x, y) \in[0,11] x[0,1]$.

On prescribing the same inflow ( $h=1$ and $F_{0}=4.5$ ), outflow and sidewall boundary conditions as done in Fig. 2.7(a) and solving the 2D system of shallow granular equations, the top panel of Fig. 2.7(b) illustrates the corresponding flow height profile for this new channel configuration. On comparison with the 2D height profile in Fig. 2.7(a), we also observe similar weak oblique jumps across the deflecting sidewalls region. Furthermore, the width-averaged 2D solution is also in perfect agreement with the solution obtained from our asymptotic theory, see the bottom panel of Fig. 2.7(b). Implying that the premise upon which our one-dimensional (1D) shallow granular theory is based, i.e. $H / W<<1$, still surprisingly holds for the case when the characteristic length scales $H=W$. More importantly, in our dimensionless $1 \mathrm{D}$ equation (2.12) we scale the channel width $W$ such that $W \in[0,1]$. For example, given a channel configuration where the contracting sidewalls span from $x=x_{m}$ to $x=x_{c}$, see Fig. 2.1, the dimensionless (scaled) width of the channel is

$$
W(x)= \begin{cases}1, & \text { if } x<=x_{m} . \\ \left(\frac{B_{c}-1}{x_{c}-x_{m}}\right)\left(x-x_{m}\right)+1, & x_{m}<x<x_{c} . \\ B_{c}, & x=x_{c} .\end{cases}
$$

The dimensionless 1D theory (2.12) is independent of the channel width as long as the angle made by contracting sidewalls is kept the same. Thus making this simple theory more effective. However, for the same $\left(F_{0}, B_{c}\right)$ pair $(4.5,0.4)$ which the theory predicts to have a stationary jump in the contraction region, see Fig. 2.6(iii), we numerically obtained an upstream moving shock (data not shown) rather than the predicted stationary jump. Although DGFEM is an ideal numerical technique to simulate flows with discontinuities, further detailed investigation is required as part of future work.

On the other hand, for subcritical flows $(F<1)$, prescribing a certain inflow height and downstream velocity at the inflow boundary and a free-flow boundary condition at the outflow boundary is not sufficient. Instead, we treat the boundary conditions using a Riemann invariant [53] and specify the height $h^{\text {in }}=1$ and $h^{\text {out }}=0.6$ at either end. As a result, the corresponding two-dimensional flow height profile for the $\left(F_{0}, B_{c}\right)$ pair $(0.3,0.85)$ is illustrated in the top panel of Fig. 2.8. Note that the choice of prescribing $\left(F_{0}, B_{c}\right)=(0.3,0.85)$ corresponds to the $\left(F_{0}, B_{c}\right)$ pair $(0.3,0.85)$ in the smooth subcritical region of Fig. 2.5. Furthermore, the numerically computed 2D DGFEM solutions are widht-averaged and compared to the solutions obtained from our 1D theory. As il- 
lustrated, the $2 \mathrm{D}$ averaged solutions are again in perfect agreement with the 1D solutions, see the bottom panel of Fig. 2.8. Thus, from Fig. 2.7 and Fig. 2.8, one can conclude that the one-dimensional asymptotic theory is in excellent agreement with the two-dimensional solutions for both sub- and supercritical flows.

\subsection{Conclusions}

\subsubsection{SUMMARY}

As a stepping stone towards analysing granular flows down rough inclined channels with downslope contracting walls - we employ the extensively utilised depth-averaged shallow $(H \ll L)$ granular equations. On further exploiting the shallowness $(H \ll W)$ aspect in the cross-slope direction, which is often associated with the flows considered here, we width-averaged the shallow granular equations, and arrived at a novel reduced one-dimensional granular hydraulic theory. For closure, we used an empirically determined constitutive friction law, previously calibrated using discrete particle simulations by Weinhart et al. [25]. By using the one-dimensional theory, we are able to compute the downstream flow profiles for any given channel opening and inclination in a quick and efficient manner. Besides this, we also predicted - for steady flows - the existence of multiple flow regimes. As a verification step, for super- and subcritical flows, the onedimensional theory is compared to the solutions obtained by numerically solving an equivalent depth-averaged shallow granular two-dimensional system of equations using DGFEM. We found an excellent agreement between the one- and two-dimensional solutions.

Overall, a time-efficient novel continuum theory has been presented, which is enabled to predict complex flow phenomena in inclined channel granular flows.

\subsubsection{FUTURE WORK}

Although, the one-dimensional model is verified for super- and subcritical flows, a thorough verification of the asymptotic theory is to be carried out for flows with steady jumps. Besides this, the one-dimensional theory is to be validated either via discrete particle simulations or experiments. In recent years, discrete particle simulations have been widely utilised to investigate inclined channel granular flows past deflecting geometries (obstacles) [e.g. 56-59] and varying basal properties [e.g. 26, 60, 61]. Similarly, with the development of state-of-the-art robust experimental setups, experimental studies are now possible to investigate both simple and complex inclined channel flow phenomena [e.g. 62-69]; hence, providing us with a variety of options for further validation of our novel theory, possibly in unsteady scenarios as well.

\subsubsection{ACKNOWLEDGEMENTS}

The authors would like to thank S. S. Shirsath, J. H. Padding and J. A. M. Kuipers for fruitful discussions and also acknowledge the Dutch Technology Foundation STW for its financial support of project 11039 Polydispersed Granular Flows over Inclined Channels and Vidi project 13472, Shaping Segregation: Advanced Modelling of Segregation and its Application to Industrial Processes. 


\section{REFERENCES}

[1] D. R. Tunuguntla, Polydisperse granular flows over inclined channels, Ph.D. thesis, Enschede (2015).

[2] D. R. Tunuguntla, I. F. C. Denissen, T. Weinhart, J. J. W. van der Vegt, S. Luding, O. Bokhove, and A. R. Thornton, Granular flow down a rough inclined channel with a linear contraction, in preparation .

[3] T. Shinbrot, A. Alexander, and F. J. Muzzio, Spontaneous chaotic granular mixing, Nature 397, 675 (1999).

[4] J. F. Davidson, D. M. Scott, P. A. Bird, O. Herbert, A. A. Powell, and H. V. M. Ramsey, Granular motion in a rotary kiln: the transition from avalanching to rolling, KONA 18, 149 (2000).

[5] S. S. Shirsath, J. T. Padding, J. A. M. Kuipers, T. W. J. Peeters, and H. J. H. Clercx, Numerical investigation of monodisperse granular flow through an inclined rotating chute, AIChE Journal 60, 3424 (2014).

[6] Y. Forterre and O. Pouliquen, Flows of dense granular media, Annu. Rev. Fluid Mech. 40, 1 (2008).

[7] R. Delannay, A. Valance, A. Mangeney, O. Roche, and P. Richard, Granular and particle-laden flows: from laboratory experiments to field observations, J. Physics D: Appl. Phys. 50, 053001 (2017).

[8] J. Dufek, The fluid mechanics of pyroclastic density currents, Annu. Rev. Fluid Mech. 48, 459 (2016).

[9] S. S. Grigorian, M. E. Eglit, and I. L. Iakimov, New statement and solution of the problem of the motion of snow avalanche, Snow, Avalanches \& Glaciers. Tr. Vysokogornogo Geofizich Inst 12, 104 (1967).

[10] S. B. Savage and K. Hutter, The motion of a finite mass of granular material down a rough incline, J. Fluid Mech. 199, 177 (1989).

[11] J. M. N. T. Gray, Y. C. Tai, and S. Noelle, Shock waves, dead zones and particle-free regions in rapid granular free-surface flows, J. Fluid Mech. 491, 161 (2003).

[12] O. Bokhove and A. R. Thornton, Shallow granular flows, H. J. Fernando (ed.) Handbook of Environmental Fluid Dynamics, Volume One: Overview and Fundamentals (2012).

[13] O. Castro-Orgaz and W. Hager, Granular flows, in Non-Hydrostatic Free Surface Flows (Springer, 2017) pp. 563-583.

[14] T. Faug, Depth-averaged analytic solutions for free-surface granular flows impacting rigid walls down inclines, Phys. Rev. E 92, 062310 (2015). 
[15] G. Saingier, S. Deboeuf, and P.-Y. Lagrée, On the front shape of an inertial granular flow down a rough incline, Phys. Fluids 28, 053302 (2016).

[16] S. Viroulet, J. L. Baker, A. N. Edwards, C. G. Johnson, C. Gjaltema, P. Clavel, and J. M. N. T. Gray, Multiple solutions for granular flow over a smooth two-dimensional bump, J. Fluid Mech. 815, 77-116 (2017).

[17] S. Mejean, T. Faug, and I. Einav, A general relation for standing normal jumps in both hydraulic and dry granular flows, J. Fluid Mech. 816, 331 (2017).

[18] K. M. Hákonardóttir and A. J. Hogg, Oblique shocks in rapid granular flows, Phys. Fluids 17, 077101 (2005).

[19] A. W. Vreman, M. Al-Tarazi, J. A. M. Kuipers, M. van Sint Annaland, and O. Bokhove, Supercritical shallow granular flow through a contraction: experiment, theory and simulation, J. Fluid Mech. 578, 233 (2007).

[20] X. Cui, J. M. N. T. Gray, and T. Jóhannesson, Deflecting dams and the formation of oblique shocks in snow avalanches at Flateyri, Iceland, J. Geophys. Res.: Earth Surface (2003-2012) 112 (2007).

[21] J. M. N. T. Gray and X. Cui, Weak, strong and detached oblique shocks in gravitydriven granular free-surface flows, Journal of Fluid Mechanics 579, 113 (2007).

[22] S. Rhebergen, O. Bokhove, and J. J. W. Van der Vegt, Discontinuous galerkin finite element method for shallow two-phase flows, Comput. Methods Appl. Mech. Engrg. 198, 819 (2009).

[23] A. Hogg and T. Jóhannesson, Avalanche defence schemes, in UK Success Stories in Industrial Mathematics (Springer, 2016) pp. 53-58.

[24] O. Pouliquen and Y. Forterre, Friction law for dense granular flows: application to the motion of a mass down a rough inclined plane, J. Fluid Mech. 453, 133 (2002).

[25] T. Weinhart, A. R. Thornton, S. Luding, and O. Bokhove, Closure relations for shallow granular flows from particle simulations, Granul. Matt. 14, 531 (2012).

[26] A. R. Thornton, T. Weinhart, S. Luding, and O. Bokhove, Frictional dependence of shallow-granular flows from discrete particle simulations, Eur. Phys. J. E 35, 1 (2012).

[27] I. Goldhirsch, Stress, stress asymmetry and couple stress: from discrete particles to continuous fields, Granul. Matt. 12, 239 (2010).

[28] T. Weinhart, A. R. Thornton, S. Luding, and O. Bokhove, From discrete particles to continuum fields near a boundary, Granul. Matt. 14, 289 (2012).

[29] D. R. Tunuguntla, A. R. Thornton, and T. Weinhart, From discrete elements to continuum fields: Extension to bidisperse systems, Comp. Part. Mech. 3, 349 (2016). 
[30] S. Deboeuf, E. Lajeunesse, O. Dauchot, and B. Andreotti, Flow rule, selfchannelization, and levees in unconfined granular flows, Phys. Rev. Lett. 97, 158303 (2006).

[31] A. te Voortwis, Closure laws for granular, shallow-layer, bi-disperse flows down an inclined chute, Masters Thesis, MSM Group, University of Twente (2013).

[32] M. J. Woodhouse, A. R. Thornton, C. G. Johnson, B. P. Kokelaar, and J. M. N. T. Gray, Segregation-induced fingering instabilities in granular free-surface flows, J. Fluid Mech. 709, 543 (2012).

[33] A. N. Edwards and N. M. Vriend, Size segregation in a granular bore, Phys. Rev. Fluids 1, 064201 (2016).

[34] J. L. Baker, C. G. Johnson, and J. M. N. T. Gray, Segregation-induced finger formation in granular free-surface flows, J. Fluid Mech. 809, 168 (2016).

[35] D. R. Tunuguntla, T. Weinhart, and A. R. Thornton, Comparing and contrasting sizebased particle segregation models, Comp. Part. Mech. 4, 387 (2017).

[36] O. Pouliquen, Scaling laws in granular flows down rough inclined planes, Phys. Fluids (1994-present) 11, 542 (1999).

[37] O. Hunger and N. R. Morgenstern, Experiments on the flow behaviour of granular materials at high velocity in an open channel, Geotechnique 34, 405 (1984).

[38] J. S. Patton, C. E. Brennen, and R. H. Sabersky, Shear flows of rapidly flowing granular materials, J. Appl. Mech. 54, 801 (1987).

[39] H. Ahn, C. E. Brennen, and R. H. Sabersky, Measurements of velocity, velocity fluctuation, density, and stresses in chute flows of granular materials, J. Appl. Mech. 58, 792 (1991).

[40] D. A. Augenstein and R. Hogg, Friction factors for powder flow, Powder Tech. 10, 43 (1974).

[41] J. W. Vallance, Experimental and Field Studies Related to the Behavior of Granular Mass Flows and the Characteristics of their Deposits, Ph.D. thesis (1994).

[42] E. Azanza, F. Chevoir, and P. Moucheront, Experimental study of collisional granular flows down an inclined plane, J. Fluid Mech. 400, 199 (1999).

[43] Y. Forterre and O. Pouliquen, Long-surface-wave instability in dense granular flows, J. Fluid Mech. 486, 21 (2003).

[44] B. Akers and O. Bokhove, Hydraulic flow through a channel contraction: Multiple steady states, Phys. fluids 20, 056601 (2008).

[45] D. D. Houghton and A. Kasahara, Nonlinear shallow fluid flow over an isolated ridge, Communications on Pure and Applied Mathematics 21, 1 (1968). 
[46] B. Akers, Shallow water flow through a contraction, Proc. GFD Fellowship Program (2005).

[47] G. B. Whitham, Linear and Nonlinear Waves (John Wiley \& Sons, 1974).

[48] D. A. Di Pietro and A. Ern, Mathematical aspects of discontinuous Galerkin methods, Vol. 69 (Springer Science \& Business Media, 2011).

[49] P. A. Tassi, O. Bokhove, and C. A. Vionnet, Space discontinuous Galerkin method for shallow water flows-kinetic and hllc flux, and potential vorticity generation, Adv. Wat. Res. 30, 998 (2007).

[50] A. Harten, P. D. Lax, and B. Van Leer, On upstream differencing and Godunov-type schemes for hyperbolic conservation laws, in Upwind and High-Resolution Schemes (Springer, 1997) pp. 53-79.

[51] L. Pesch, A. Bell, H. Sollie, V. R. Ambati, O. Bokhove, and J. J. W. Van Der Vegt, hpGEM-a software framework for discontinuous Galerkin finite element methods, ACM Trans. Math. Softw. 33, 23 (2007).

[52] S. Nurijanyan, Discrete and continuous Hamiltonian systems for wave modelling, Ph.D. thesis, University of Twente, Enschede (2013).

[53] M.-O. Bristeau and B. Coussin, Boundary conditions for the shallow water equations solved by kinetic schemes, Ph.D. thesis, INRIA (2001).

[54] V. R. Ambati and O. Bokhove, Space-time discontinuous Galerkin discretization of rotating shallow water equations, J. Comp. Phys. 225, 1233 (2007).

[55] P. Jop, Y. Forterre, and O. Pouliquen, Crucial role of sidewalls in granular surface flows: consequences for the rheology, J. Fluid Mech. 541, 167 (2005).

[56] T. Faug, R. Beguin, and B. Chanut, Mean steady granular force on a wall overflowed by free-surface gravity-driven dense flows, Phys. Rev. E 80, 021305 (2009).

[57] S. H. Chou, L. S. Lu, and S. S. Hsiau, DEM simulation of oblique shocks in gravitydriven granular flows with wedge obstacles, Granul. Matt. 14, 719 (2012).

[58] P. A. Moysey, N. V. R. Rao, and M. H. I. Baird, Dynamic coefficient of friction and granular drag force in dense particle flows: Experiments and dem simulations, Powder Technol. 248, 54 (2013).

[59] C.-Y. Kuo, L.-T. Sheng, S.-Y. Chiu, Y.-Z. Yang, Y.-C. Tai, and S.-S. Hsiau, Measurement and discrete element simulation of a fixed-obstacle disturbed rapid granular chute flow, Phys. Fluids 27, 013305 (2015).

[60] L. Jing, C. Y. Kwok, Y. F. Leung, and Y. D. Sobral, Characterization of base roughness for granular chute flows, Phys. Rev. E 94, 052901 (2016).

[61] S. Bharathraj and V. Kumaran, Effect of base topography on dynamics and transition in a dense granular flow, J. Fluid Mech. 832, 600 (2017). 
[62] D. Takagi, J. N. McElwaine, and H. E. Huppert, Shallow granular flows, Phys. Rev. E 83, 031306 (2011).

[63] S. S. Shirsath, J. T. Padding, N. G. Deen, H. J. H. Clercx, and J. A. M. Kuipers, Experimental study of monodisperse granular flow through an inclined rotating chute, Powder Technol. 246, 235 (2013).

[64] T. Haas, L. Braat, J. R. F. W. Leuven, I. R. Lokhorst, and M. G. Kleinhans, Effects of debris flow composition on runout, depositional mechanisms, and deposit morphology in laboratory experiments, J. Geophys. Res.: Earth Surface 120, 1949 (2015).

[65] T. Faug, P. Childs, E. Wyburn, and I. Einav, Standing jumps in shallow granular flows down smooth inclines, Phys. Fluids 27, 073304 (2015).

[66] N. Sanvitale and E. T. Bowman, Using piv to measure granular temperature in saturated unsteady polydisperse granular flows, Granul. Matt. 18, 1 (2016).

[67] D. Gollin, W. Brevis, E. T. Bowman, and P. Shepley, Performance of piv and ptv for granular flow measurements, Granul. Matt. 19, 42 (2017).

[68] J. M. Gong, H. Yang, S. H. Lin, R. Li, and V. Zivkovic, Spatial filtering velocimetry for surface velocity measurement of granular flow, Powder Technol. 324, 76 (2017).

[69] F. Guillard, B. Marks, and I. Einav, Dynamic $x$-ray radiography reveals particle size and shape orientation fields during granular flow, Sci. Rep. 7, 8155 (2017). 


\section{APPENDiX}

\section{A. Details of Derivation 1 D Shallow GRANUlar MODEL}

Before we derive and arrive at the 1D shallow granular model, we first consider the required kinematic boundary conditions.

\section{A.1. KINEMATIC BOUNDARY CONDITIONS}

Given the symmetry of the channel sidewalls, the left and right sidewalls are defined as $y= \pm \frac{W(x, t)}{2}$. Note that $W(x, t)$ here represents the profile of the channel sidewall as a function of space and time instead of space alone. Given these definitions, the kinematic boundary conditions must hold at, both, the left and right sidewall. Thus, implying

$$
\begin{gathered}
y \pm \frac{W(x, t)}{2}=0 \rightarrow \frac{d}{d t}\left(y \pm \frac{W(x, t)}{2}\right)=0 \\
\frac{\partial}{\partial t}\left(y \pm \frac{W(x, t)}{2}\right)+\frac{\partial}{\partial x}\left(y \pm \frac{W(x, t)}{2}\right) u(x, \pm W / 2, t)=0 .
\end{gathered}
$$

Now if the left and right sidewalls are defined as $y=-\frac{W(x, t)}{2}$ and $y=\frac{W(x, t)}{2}$ and are further represented using superscripts ' $l$ ' and ' $r$ ', from (2.32), the corresponding boundary conditions can be restated as

$$
v^{l}+\frac{W_{t}}{2}+u^{l} \frac{W_{x}}{2}=0 \stackrel{\text { on multiplying } h^{l}}{=} h^{l} v^{l}+h^{l} \frac{W_{t}}{2}+h^{l} u^{l} \frac{W_{x}}{2}=0 .
$$

and similarly

$$
v^{r}-\frac{W_{t}}{2}-u^{r} \frac{W_{x}}{2}=0 \stackrel{\text { on multiplying } h^{r}}{=} h^{r} v^{r}-h^{r} \frac{W_{t}}{2}-h^{r} u^{r} \frac{W_{x}}{2}=0 .
$$

Assuming that the sidewalls are impermeable and stationary, we obtain a more familiar boundary condition $\vec{u} \cdot \vec{n}=0$, where $\vec{n}$ is the outward normal of a stationary sidewall.

\section{A.2. WIDTH-AVERAGING}

Utilising the boundary conditions (2.33) and (2.34), the depth-averaged dimensional balance laws (2.1) are width averaged from $-W(x, t) / 2$ to $W(x, t) / 2$. First, we begin by integrating the depth-averaged mass balance equation $(2.1)_{1}$,

$$
\int_{-W / 2}^{W / 2}\left[\frac{\partial h}{\partial t}+\frac{\partial(h u)}{\partial x}+\frac{\partial(h v)}{\partial y}\right] d y=0
$$


Applying Leibnitz' rule on each term of (2.35) results in

$$
\begin{aligned}
& \text { (i) } \int_{-W / 2}^{W / 2} \frac{\partial h}{\partial t} d y=\frac{\partial}{\partial t} \int_{-W / 2}^{W / 2} h d y-\left[\frac{\partial y}{\partial t} h\right]_{-W / 2}^{W / 2} \cdot \\
& \text { (ii) } \int_{-W / 2}^{W / 2} \frac{\partial(h u)}{\partial x} d y=\frac{\partial}{\partial x} \int_{-W / 2}^{W / 2}(h u) d y-\left[\frac{\partial y}{\partial x}(h u)\right]_{-W / 2}^{W / 2} . \\
& \text { (ii i) } \int_{-W / 2}^{W / 2} \frac{\partial(h v)}{\partial y} d y=[h v]_{-W / 2}^{W / 2}=\frac{h^{l}}{2} W_{t}+\frac{h^{l} u^{l}}{2} W_{x}+\frac{h^{r}}{2} W_{t}+\frac{h^{r} u^{r}}{2} W_{x} .
\end{aligned}
$$

By definition, the width-averaged value $\bar{f}$ of a variable $f$ is

$$
\bar{f}(x, t)=\frac{1}{W} \int_{-W / 2}^{W / 2} f(x, y, t) d y .
$$

Thereby, on substituting this definition into (2.36) and then dropping the bars, the resulting width-averaged mass balance equation is

$$
\frac{\partial(h W)}{\partial t}+\frac{\partial(h u W)}{\partial x}=0
$$

Given the width- and depth-averaged mass balance equation, we perform the same steps as done in (2.35) - (2.37) for width-averaging the depth-averaged momentum balance equations, respectively. To begin with, we consider integrating the downslope depthaveraged momentum balance equation (2.1), which for no varying basal topography is

$$
\int_{-W / 2}^{W / 2}\left[\frac{\partial(h u)}{\partial t}+\frac{\partial}{\partial x}\left(h u^{2}+K g_{n} \frac{h^{2}}{2}\right)+\frac{\partial}{\partial y}(h u v)=h\left(\tan \theta-\mu \frac{u}{|\vec{u}|}\right) g_{n}\right] d y .
$$

Again, on applying the Leibnitz' integral rule on each of the terms stated in (2.39) and utilising the boundary conditions (2.33) and (2.34), we have

$$
\begin{aligned}
& \text { (i) } \int_{-W / 2}^{W / 2} \frac{\partial(h u)}{\partial t} d y=\frac{\partial}{\partial t} \int_{-W / 2}^{W / 2}(h u)(x, y, t) d y-\left[\frac{\partial y}{\partial t}(h u)(x, y, t)\right]_{-W / 2}^{W / 2} . \\
& \text { (ii) } \int_{-W / 2}^{W / 2} \frac{\partial\left(h u^{2}+K \frac{h^{2}}{2} g_{n}\right)}{\partial x} d y=\frac{\partial}{\partial x} \int_{-W / 2}^{W / 2}\left(h u^{2}+K \frac{h^{2}}{2} g_{n}\right) d y-\ldots \\
& \quad \ldots\left[\frac{\partial y}{\partial x}\left(h u^{2}+K \frac{h^{2}}{2} g_{n}\right)\right]_{-W / 2}^{W / 2} . \\
& \text { (iii) } \int_{-W / 2}^{W / 2} \frac{\partial(h u v)}{\partial y} d y=[h u v]_{-W / 2}^{W / 2}=\frac{h^{l} u^{l}+h^{r} u^{r}}{2} W_{t}+\frac{h^{l}\left(u^{l}\right)^{2}+h^{r}\left(u^{r}\right)^{2}}{2} W_{x} . \\
& \text { (iv) } \int_{-W / 2}^{W / 2} h\left(\tan \theta-\mu \frac{u}{|\vec{u}|}\right) g_{n} d y=h W\left(\tan \theta-\mu \frac{u}{|\vec{u}|}\right) g_{n} .
\end{aligned}
$$

On substituting the definition of width-averaging (2.37) in the above results (2.40) and then dropping the bars, allows us to restate (2.39) as

$$
\frac{\partial}{\partial t}(h u W)+\frac{\partial}{\partial x}\left(h u^{2} W+K W \frac{h^{2}}{2} g_{n}\right)-\left[K W_{x} \frac{h^{2}}{2} g_{n}\right]_{-W / 2}^{W / 2}=h W\left(\tan \theta-\mu \frac{u}{|\vec{u}|}\right) g_{n} .
$$


Additionally, by utilising the chain rule of differentiation, $h^{r}=h^{l}=h(x, t)$ and the widthand depth-averaged mass balance (2.38), leads to further simplification of (2.41), resulting in the width- and depth-averaged downslope momentum balance

$$
\begin{gathered}
(\underbrace{\left.(h W)_{t}+(h u W)_{x}\right)}_{\text {mass balance }} u+h W u_{t}+(h u W) u_{x}+g_{n} K h W h_{x}=g_{n} h W\left(\tan \theta-\mu \frac{u}{|\vec{u}|}\right), \\
u_{t}+u u_{x}+K g_{n} h_{x}=g_{n}\left(\tan \theta-\mu \frac{u}{|\vec{u}|}\right) .
\end{gathered}
$$

Note that in (2.41) and (2.42), $\vec{u}$ is width- and depth-averaged velocity vector, while $u$ is its downstream velocity component.

\section{A.3. DERIVED RELATIONS}

By substituting the definition corresponding to the local Froude number $F=F_{l} u / \sqrt{h}$, into the mass balance equation (2.38), we have for steady state, the mass flux

$$
\begin{gathered}
h u W=Q \Rightarrow u=\frac{Q}{h W} \text { and } \\
h=F_{l}^{2} \frac{u^{2}}{F^{2}} \rightarrow h=\frac{Q^{2} F_{l}^{2}}{h^{2} W^{2} F^{2}} \rightarrow h^{3}=\frac{Q^{2} F_{l}^{2}}{W^{2} F^{2}} \Rightarrow h=\left(\frac{Q F_{l}}{W F}\right)^{2 / 3} .
\end{gathered}
$$

From (2.43), we further derive

$$
\begin{aligned}
\frac{d h}{d x} & =\frac{2}{3}\left(\frac{Q F_{l}}{W F}\right)^{-1 / 3} \frac{d}{d x}\left(\frac{Q F_{l}}{W F}\right) \rightarrow \frac{d h}{d x}=\frac{2}{3}\left(\frac{Q F_{l}}{W F}\right)^{-1 / 3}\left(\frac{Q F_{l}}{W F}\right) W F \frac{d}{d x}\left(\frac{1}{W F}\right) \\
& \rightarrow \frac{d h}{d x}=\frac{2}{3}\left(\frac{Q F_{l}}{W F}\right)^{2 / 3}\left[\frac{-W F_{x}-W_{x} F}{W F}\right] \Rightarrow \frac{d h}{d x}=-\frac{2}{3}\left[\frac{h}{F} F_{x}+\frac{h}{W} W_{x}\right] .
\end{aligned}
$$

\section{A.4. Froude FUnCTION}

For $K=1$ and substituting (2.43) and (2.44) in the 1D momentum equation (2.42), we have

$$
\frac{d}{d x}\left[\left(\frac{1}{2} F^{2}+1\right) h\right]=(\tan \theta-\mu(h, F)) \rightarrow h F \frac{d F}{d x}=-\frac{F^{2}}{2} \frac{d h}{d x}-\frac{d h}{d x}+(\tan \theta-\mu(h, F)) .
$$

Substituting (2.44) in the above equation and on further simplifications, we have

$$
\begin{gathered}
h F \frac{d F}{d x}=\frac{2}{3}\left[h\left(\frac{F^{2}}{2}+1\right)\right]\left[\frac{1}{F} \frac{d F}{d x}+\frac{1}{W} \frac{d W}{d x}\right]+(\tan \theta-\mu(h, F)), \\
\left(1-\frac{F^{2}+2}{3 F^{2}}\right) \frac{d F}{d x}=\left(\frac{F^{2}+2}{3 F}\right) \frac{1}{W} \frac{d W}{d x}+\frac{1}{h F}(\tan \theta-\mu(h, F)), \\
\frac{d F}{d x}=\frac{1}{2} \frac{\left(F^{2}+2\right) F}{F^{2}-1} \frac{d(\ln W)}{d x}+\frac{3}{2} \frac{F}{h\left(F^{2}-1\right)}(\tan \theta-\mu(h, F)) .
\end{gathered}
$$

Substituting (2.43) in $(2.45)_{3}$, results in a 1D momentum balance expressed in terms of the local Froude number as variable,

$$
\frac{d F}{d x}=\frac{1}{2}\left(\frac{F^{2}+2}{F^{2}-1}\right) F \frac{d(\ln W)}{d x}+\frac{3}{2} \frac{1}{\left(Q F_{l}\right)^{2 / 3}}\left(\frac{F^{5 / 3}}{F^{2}-1}\right) W^{2 / 3}(\tan \theta-\mu(h, F)) .
$$




\section{A.5. AnAlYtic SOlution FOR INVISCID FlOW}

For the inviscid case, equation (2.12) can be written as

$$
\frac{d F}{d x}=\frac{1}{2}\left(\frac{F^{2}+2}{F^{2}-1}\right) \frac{F}{W} \frac{d W}{d x},
$$

which when analytically integrated with respect to $x$, from the channel upstream position $x_{l}$ to some point channel downstream yields

$$
\begin{gathered}
\int_{F_{l}}^{F} \frac{2\left(F^{2}-1\right)}{\left(F^{2}+2\right) F} d F=\int_{W_{0}}^{W} \frac{1}{W} d W \stackrel{F^{2}=\mathscr{F}}{\Longrightarrow} \int_{F_{l}^{2}}^{F^{2}} \frac{\mathscr{F}-1}{(\mathscr{F}+2) \mathscr{F}} d \mathscr{F}=[\ln W]_{W_{0}}^{W}, \\
{\left[\frac{3}{2} \ln (\mathscr{F}+2)-\frac{\ln (\mathscr{F})}{2}\right]_{F_{l}^{2}}^{F^{2}}=[\ln W]_{W_{0}}^{W} \Rightarrow\left[\ln \left(\frac{(\mathscr{F}+2)^{3 / 2}}{\mathscr{F}^{1 / 2}}\right)\right]_{F_{l}^{2}}^{F^{2}}=[\ln W]_{W_{0}}^{W},} \\
\frac{F_{l}}{F}\left(\frac{2+F^{2}}{2+F_{l}^{2}}\right)^{3 / 2}=\frac{W}{W_{0}}
\end{gathered}
$$

\section{B. REGULARISATION}

Here, we regularise the following equation

$$
\underbrace{\frac{2}{3}\left(\frac{F^{2}-1}{F^{2}}\right) \frac{d F}{d x}}_{\text {LHS }}=\underbrace{\left(\frac{F^{2}+2}{3 F}\right) \frac{d(\ln W)}{d x}+\frac{F^{-1 / 3} W^{2 / 3}}{\left(Q F_{l}\right)^{2 / 3}}(\tan \theta-\mu(F))}_{R H S}
$$

and then solve the limit case

$$
\lim _{F\left(x_{\text {cnew }}\right) \rightarrow 1} \frac{2}{3}\left(\frac{F^{2}-1}{F^{2}}\right) \frac{d F}{d x}=\left(\frac{F^{2}+2}{3 F}\right) \frac{d(\ln W)}{d x}+\frac{F^{-1 / 3} W^{2 / 3}}{\left(Q F_{l}\right)^{2 / 3}}(\tan \theta-\mu(F)),
$$

for the inviscid and frictional flow case. Note that $x_{\text {cnew }}$ is the new contraction exit that is determined via regularisation, see figure 2.B.1, considered as follows.

\section{B.1. INVISCID FLOWS $(\mu \approx \tan \theta)$}

The slope of the contraction $\widehat{\vec{\gamma}}$ and the normal to the contraction $\widehat{\vec{n}}$ are given by

$$
\widehat{\vec{\gamma}}=\left[\begin{array}{c}
x_{c} \\
-\left(W_{0}-W_{c}\right)
\end{array}\right], \quad \widehat{\vec{n}}=\left[\begin{array}{c}
W_{0}-W_{c} \\
x_{c}
\end{array}\right],
$$

where ' ${ }^{\wedge}$ denotes a dimensionless quantity. The equation of a line with position vector $\left[\begin{array}{c}x_{c} \\ W_{c}\end{array}\right]$ and direction vector $\hat{\vec{n}}$ is

$$
\begin{gathered}
{\left[\begin{array}{c}
x \\
W
\end{array}\right]=\lambda\left[\begin{array}{c}
W_{0}-W_{c} \\
x_{c}
\end{array}\right]+\left[\begin{array}{c}
x_{c} \\
W_{c}
\end{array}\right],} \\
{\left[\begin{array}{c}
x-x_{c} \\
W-W_{c}
\end{array}\right]=\lambda\left[\begin{array}{c}
W_{0}-W_{c} \\
x_{c}
\end{array}\right] .}
\end{gathered}
$$




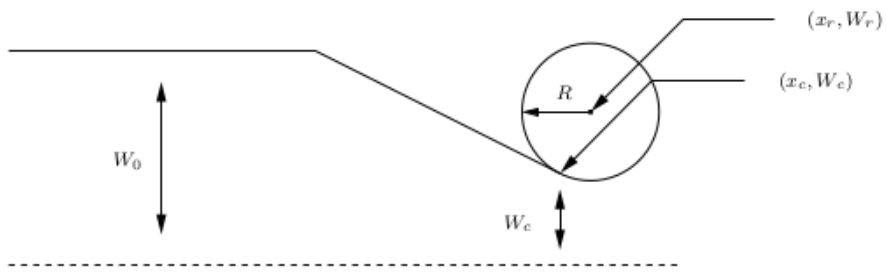

Figure 2.B.1: $x_{c}$ in the initial downstream location of the contraction exit. $x_{r}$ is the location for the centre of the circle used to regularise. For the inviscid case, after regularisation, $x_{r}$ turns out to be the new location for the contraction exit.

As $x_{r}, W_{r}$ lie on the line (2.52),

$$
\left[\begin{array}{c}
x_{r}-x_{c} \\
W_{r}-W_{c}
\end{array}\right]=\lambda_{r}\left[\begin{array}{c}
W_{0}-W_{c} \\
x_{c}
\end{array}\right]
$$

The value of $\lambda_{r}$ is determined by considering the distance between $\left(x_{c}, W_{c}\right)$ and $\left(x_{r}, W_{r}\right)$, which is

$$
\left(x_{r}-x_{c}\right)^{2}+\left(W_{r}-W_{c}\right)^{2}=R^{2} \text {. }
$$

Using (2.53) together with (2.54), gives

$$
\lambda_{r}^{2}\left[\left(W_{0}-W_{c}\right)^{2}+x_{c}^{2}\right]=R^{2} \rightarrow \lambda_{r}=\frac{R}{\sqrt{\left(W_{0}-W_{c}\right)^{2}+x_{c}^{2}}} .
$$

Now as $\lambda_{r}$ is determined, the center of the circle for regularisation is computed using (2.53). Thereby, on utilising the equation of a circle, we have the extended sidewall profile as $W(x)=W_{r}-\sqrt{R^{2}-\left(x-x_{r}\right)^{2}}$, with $x_{c}<x<\left(x_{r}+R\right), W^{\prime}=\frac{x-x_{r}}{\sqrt{R^{2}-\left(x-x_{r}\right)^{2}}}$ and $W^{\prime \prime}=\frac{R^{2}}{\left(R^{2}-\left(x-x_{r}\right)^{2}\right)^{3 / 2}}$.

Given the expressions corresponding to the extended sidewall are determined as above, for the inviscid case, the right hand side of the ODE

$$
\frac{2}{3}\left(\frac{F^{2}-1}{F^{2}}\right) \frac{d F}{d x}=\left(\frac{F^{2}+2}{3 F}\right) \frac{1}{W} \frac{d W}{d x},
$$

becomes zero when $d W / d x=0$, which is when $x=x_{r}$. Thus, implying that (i) the critical condition of Houghton and Kasahara [1] is evaluated at $x_{r}$ such that $F\left(x_{r}\right)=1$ and (ii) $W\left(x_{r}\right)=W_{r}-R$. Given the ODE is regularised for the inviscid case, we solve the limit's problem

$$
\lim _{F\left(x_{\text {cnew }}\right) \rightarrow 1} \frac{2}{3}\left(\frac{F^{2}-1}{F^{2}}\right) \frac{d F}{d x}=\left(\frac{F^{2}+2}{3 F}\right) \frac{d(\ln W)}{d x},
$$


as follows. Taylor expansion of $F(x)$ and $W(x)$ about $x_{r}$ gives

$$
\begin{array}{ccc}
F & = & 1+F_{r}^{\prime}\left(x-x_{r}\right)+\frac{1}{2} F_{r}^{\prime \prime}\left(x-x_{r}\right)^{2}+\ldots . ., \\
F^{2} & = & 1+2 F_{r}^{\prime}\left(x-x_{r}\right)+O\left(x-x_{r}\right)^{2} \\
W & = & W_{r}-R+W_{r}^{\prime}\left(x-x_{r}\right)+W_{r}^{\prime \prime}\left(x-x_{r}\right)^{2}+\ldots \ldots . \\
W^{\prime} & = & W_{r}^{\prime \prime}\left(x-x_{r}\right)+O\left(x-x_{r}\right)^{2}
\end{array}
$$

where $F^{\prime}\left(x_{r}\right)=F_{r}^{\prime}, F^{\prime \prime}\left(x_{r}\right)=F_{r}^{\prime \prime}, W^{\prime}\left(x_{r}\right)=W_{r}^{\prime}, W^{\prime \prime}\left(x_{r}\right)=W_{r}^{\prime \prime}=\frac{1}{R}$, with the primes denoting the order of differentiation. Substituting (2.58) in (2.56) leads to

$$
\frac{4}{3}\left(F_{r}^{\prime}\right)^{2}\left(x-x_{r}\right) \approx \frac{W_{r}^{\prime \prime}\left(x-x_{r}\right)}{W_{r}-R} \rightarrow F_{r}^{\prime}=\sqrt{\frac{3}{4\left(W_{r}-R\right) R}} .
$$

\section{B.2. FRICTIONAL FLOWS (VISCID)}

To regularise the ODE (2.49), for the viscid case, we restate the ODE as

$$
\left(\frac{F^{2}-1}{F}\right) \frac{d F}{d x}=\left(\frac{F^{2}+2}{2}\right) \frac{d \ln (W)}{d x}+\frac{3}{2} \frac{[\tan \theta-\mu(F)]}{\left(Q F_{l}\right)^{2 / 3}} \frac{W^{2 / 3} F^{5 / 3}}{\left(F^{2}-1\right)}
$$

where the expression for the constitutive friction law is

$$
\mu(F)=\tan \delta_{1}+\frac{\tan \delta_{2}-\tan \delta_{2}}{\left[v\left(\frac{F_{l} Q}{W F}\right)^{2 / 3} \frac{1}{F-\gamma}+1\right]},
$$

with $v=\beta_{\lambda} / A d$. For more details concerning the friction law, see section 2.2.1 or Pouliquen and Forterre [2]. However, as mentioned earlier, the ODE does not hold at the contraction exit. Thereby, here we determine the point at which the ODE holds for the viscid case. To determine the new contraction exit, $x_{c n e w}$, we utilise the bisection method with the starting interval as $\left[x_{r}, x_{r}+\Delta x\right]$. The limits are chosen such that the right-hand side of (2.60) has opposite signs at these points. Moreover, we choose $x_{r}$ as the lower limit because it is the point at which the ODE holds for the inviscid case, i.e. where $d W / d x=0$, see Appendix 2.B.1.

Given the ODE is now regularised for the viscid case, we further solve the limit's problem (2.50) utilising the same Taylor expansion approach that was followed in Appendix 2.B.1. Thereby, we denote the new contraction exit $x_{c n e w}$ as $x_{e}$ and, for simplicity, define the values of some variables at $x_{e}$ as $F_{e}=F\left(x_{e}\right)=1, F_{e}^{\prime}=F^{\prime}\left(x_{e}\right), W_{e}=W\left(x_{e}\right), W_{e}^{\prime}=$ $W^{\prime}\left(x_{e}\right), W_{e}^{\prime \prime}=W^{\prime \prime}\left(x_{e}\right)$. Given these definitions, we Taylor expand the primary variables 
$F(x), W(x)$ and the secondary variables $W^{\prime}, F^{2}, 1 / F$ about $x_{e}$ as follows

$$
\begin{array}{ccc}
F & = & 1+F_{e}^{\prime}\left(x-x_{e}\right)+\frac{1}{2} F_{e}^{\prime \prime}\left(x-x_{e}\right)^{2}+O\left(\left(x-x_{e}\right)^{3}\right), \\
F^{\prime} & = & F_{e}^{\prime}+F_{e}^{\prime \prime}\left(x-x_{e}\right)+O\left(\left(x-x_{e}\right)^{2}\right), \\
F^{2} & = & 1+2 F_{e}^{\prime}\left(x-x_{e}\right)+\left[\left(F_{e}^{\prime}\right)^{2}+F_{e}^{\prime \prime}\right]\left(x-x_{e}\right)^{2}+O\left(\left(x-x_{e}\right)^{3}\right), \\
F^{2}-1 & = & 2 F_{e}^{\prime}\left(x-x_{e}\right)+O\left(\left(x-x_{e}\right)^{2}\right), \\
\frac{1}{F} & = & 1-F_{e}^{\prime}\left(x-x_{e}\right)+O\left(\left(x-x_{e}\right)^{2}\right), \\
W & = & W_{e}+W_{e}^{\prime}\left(x-x_{e}\right)+\frac{W_{e}^{\prime \prime}}{2}\left(x-x_{e}\right)^{2}+O\left(\left(x-x_{e}\right)^{3}\right), \\
W^{\prime} & = & W_{e}^{\prime}+W_{e}^{\prime \prime}\left(x-x_{e}\right)+O\left(\left(x-x_{e}\right)^{2}\right) .
\end{array}
$$

Given the above definitions and expansions at hand, we Taylor expand each term of (2.60) about $x_{e}$ as follows

\section{LHS of (2.60)}

$$
\begin{aligned}
\left(\frac{F^{2}-1}{F}\right) \frac{d F}{d x} & \approx 2 F_{e}^{\prime}\left(x-x_{e}\right)\left(1-F_{e}^{\prime}\left(x-x_{e}\right)\right)\left(F_{e}^{\prime}+F_{e}^{\prime \prime}\left(x-x_{e}\right)\right) \\
& \approx\left(2 F_{e}^{\prime}\left(x-x_{e}\right)-2\left(F_{e}^{\prime}\right)^{2}\left(x-x_{e}\right)^{2}\right)\left(F_{e}^{\prime}+F_{e}^{\prime \prime}\left(x-x_{e}\right)\right) \\
& \approx \quad 2\left(F_{e}^{\prime}\right)^{2}\left(x-x_{e}\right)+O\left(\left(x-x_{e}\right)^{2}\right) .
\end{aligned}
$$

First term on the RHS of (2.60)

$$
\begin{gathered}
\left(\frac{F^{2}+2}{2}\right) \frac{1}{W} \frac{d W}{d x} \approx\left(\frac{3+2 F_{e}^{\prime}\left(x-x_{e}\right)}{2}\right)\left(\frac{1}{W_{e}}-\frac{W_{e}^{\prime}}{W_{e}^{2}}\left(x-x_{e}\right)\right) \ldots \\
\ldots\left(W_{e}^{\prime}+W_{e}^{\prime \prime}\left(x-x_{e}\right)\right), \\
\approx \frac{3}{2} \frac{W_{e}^{\prime}}{W_{e}}+\left(x-x_{e}\right)\left(\frac{F_{e}^{\prime} W_{e}^{\prime}}{W_{e}}-\frac{3}{2}\left(\frac{W_{e}^{\prime}}{W_{e}}\right)^{2}+\frac{2+F_{e}^{2}}{2} \frac{W_{e}^{\prime \prime}}{W_{e}}\right) \\
+O\left(\left(x-x_{e}\right)^{2}\right) .
\end{gathered}
$$

However, in order to Taylor expand the second term on the RHS of (2.60)

$$
\frac{3}{2}\left(\frac{W}{F_{l} Q}\right)^{2 / 3} F^{2 / 3}\left[\tan \theta-\tan \delta_{1}-\frac{\tan \delta_{2}-\tan \delta_{1}}{v\left(\frac{F_{l} Q}{W}\right)^{2 / 3}\left(\frac{1}{F}\right)^{2 / 3} \frac{1}{F-\gamma}+1}\right],
$$

we first expand the following variables about $x_{e}$, which on ignoring the second and 
higher order terms are

$$
\begin{array}{cccc}
W^{2 / 3} & \approx & \left(W_{e}+W_{e}^{\prime}\left(x-x_{e}\right)+O\left(x-x_{e}\right)^{2}\right)^{2 / 3} \approx W_{e}^{2 / 3}+\frac{2}{3} W_{e}^{-1 / 3} W_{e}^{\prime}\left(x-x_{e}\right) \\
\frac{1}{W^{2 / 3}} & \approx & \frac{1}{W_{e}^{2 / 3}} \frac{1}{\left(1+\frac{2}{3} \frac{W_{e}^{\prime}}{W_{e}}\left(x-x_{e}\right)\right)} \\
\frac{F^{2 / 3}}{F^{2 / 3}} & \approx & \left(1+F_{e}^{\prime}\left(x-x_{e}\right)+O\left(x-x_{e}\right)^{2}\right)^{2 / 3} \approx 1+\frac{2}{3} F_{e}^{\prime}\left(x-x_{e}\right) ; \\
\frac{1}{F-\gamma} & \approx & \frac{1}{1+F_{e}^{\prime}\left(x-x_{e}\right)-\gamma}+O\left(x-x_{e}\right)^{2} \approx \frac{1}{1-\gamma} \frac{1}{\left(1+\frac{F_{e}^{\prime}\left(x-x_{e}\right)}{1-\gamma}\right)} \\
& \approx & \frac{1}{1-\gamma}\left(1-\frac{F_{e}^{\prime}\left(x-x_{e}\right)}{1-\gamma}\right) \approx \frac{1}{1-\gamma}-\frac{F_{e}^{\prime}\left(x-x_{e}\right)}{(1-\gamma)^{2}} .
\end{array}
$$

As a result, in order to expand (2.65), we utilise the expansions (2.66) to first Taylor expand the below term about $x_{e}$

$$
v\left(F_{l} Q\right)^{2 / 3} \frac{1}{W^{2 / 3}} \frac{1}{F^{2 / 3}} \frac{1}{(F-\gamma)}+1,
$$

which on neglecting higher order terms is

$$
\begin{aligned}
& \nu\left(F_{l} Q\right)^{2 / 3} \frac{1}{W^{2 / 3}} \frac{1}{F^{2 / 3}} \frac{1}{(F-\gamma)}+1 \\
& \approx v \frac{\left(F_{l} Q\right)^{2 / 3}}{W_{e}^{2 / 3}}\left(1-\frac{2}{3} \frac{W_{e}^{\prime}}{W_{e}}\left(x-x_{e}\right)\right)\left(1-\frac{2}{3} F_{e}^{\prime}\left(x-x_{e}\right)\right) \frac{1}{1-\gamma}\left(1-\frac{F_{e}^{\prime}\left(x-x_{e}\right)}{1-\gamma}\right)+1, \\
& \approx v \frac{\left(F_{l} Q\right)^{2 / 3}}{W_{e}^{2 / 3}} \frac{1}{1-\gamma}\left(1-\frac{2}{3} F_{e}^{\prime}\left(x-x_{e}\right)-\frac{2}{3} \frac{W_{e}^{\prime}}{W_{e}}\left(x-x_{e}\right)-\frac{F_{e}^{\prime}\left(x-x_{e}\right)}{1-\gamma}\right)+1 .
\end{aligned}
$$

Now given the expansion (2.68), we Taylor expand the term

$$
\left(v\left(F_{l} Q\right)^{2 / 3} \frac{1}{W^{2 / 3}} \frac{1}{F^{2 / 3}} \frac{1}{(F-\gamma)}+1\right)^{-1},
$$

as

$$
\begin{aligned}
& \left(\nu\left(F_{l} Q\right)^{2 / 3} \frac{1}{W^{2 / 3}} \frac{1}{F^{2 / 3}} \frac{1}{(F-\gamma)}+1\right)^{-1} \\
& \approx \frac{1}{\nu \frac{\left(F_{l} Q\right)^{2 / 3}}{W_{e}^{2 / 3}} \frac{1}{1-\gamma}+1}\left(1+\frac{v \frac{\left(F_{l} Q\right)^{2 / 3}}{W_{e}^{2 / 3}} \frac{1}{1-\gamma}\left(\frac{2}{3} F_{e}^{\prime}+\frac{2}{3} \frac{W_{e}^{\prime}}{W_{e}}+\frac{F_{e}^{\prime}}{1-\gamma}\right)\left(x-x_{e}\right)}{\nu \frac{\left(F_{l} Q\right)^{2 / 3}}{W_{e}^{2 / 3}} \frac{1}{1-\gamma}+1}\right),
\end{aligned}
$$


which on further simplification results in

$$
\begin{aligned}
& \frac{1}{v\left(\frac{F_{l} Q}{W}\right)^{2 / 3}\left(\frac{1}{F}\right)^{2 / 3} \frac{1}{(F-\gamma)}+1} \\
& \approx \frac{1}{v\left(\frac{F_{l} Q}{W_{e}}\right)^{2 / 3} \frac{1}{(1-\gamma)}+1}+\frac{v\left(F_{l} Q\right)^{2 / 3}\left(x-x_{e}\right)}{\left(v\left(\frac{F_{l} Q}{W_{e}}\right)^{2 / 3} \frac{1}{(1-\gamma)}+1\right)^{2}} \\
& *\left(\frac{2}{3} \frac{W_{e}^{\prime}}{W_{e}^{5 / 3}\left(F_{e}-\gamma\right)}+\frac{2}{3} \frac{F_{e}^{\prime}}{W_{e}^{2 / 3}(1+\gamma)}+\frac{F_{e}^{\prime}}{W_{e}^{2 / 3}\left(F_{e}-\gamma\right)^{2}}\right) .
\end{aligned}
$$

Although the above expansion (2.71) forms a major part of (2.65), we further Taylor expand the following variable about $x_{e}$ such that

$$
\begin{gathered}
W^{2 / 3} F^{2 / 3} \approx\left(W_{e}^{2 / 3}+\frac{2}{3} W_{e}^{-1 / 3} W_{e}^{\prime}\left(x-x_{e}\right)\right)\left(1+\frac{2}{3} F_{e}^{\prime}\left(x-x_{e}\right)\right) \\
\approx W_{e}^{2 / 3}+\frac{2}{3} W_{e}^{2 / 3} F_{e}^{\prime}\left(x-x_{e}\right)+\frac{2}{3} W_{e}^{-1 / 3} W_{e}^{\prime}\left(x-x_{e}\right)+O\left(\left(x-x_{e}\right)^{2}\right) .
\end{gathered}
$$

Thus, given (2.71) and (2.72), the Taylor expansion of (2.65) on ignoring higher order terms is

$$
\begin{gathered}
\frac{3}{2}\left(\frac{W}{F_{l} Q}\right)^{2 / 3} F^{2 / 3}\left(\tan \theta-\tan \delta_{1}-\frac{\tan \delta_{2}-\tan \delta_{1}}{v\left(\frac{F_{l} Q}{W}\right)^{2 / 3}\left(\frac{1}{F}\right)^{2 / 3} \frac{1}{F-\gamma}+1}\right) \\
\approx \frac{3}{2}\left(\frac{1}{F_{l} Q}\right)^{2 / 3}\left(W_{e}^{2 / 3}+\frac{2}{3} W_{e}^{2 / 3} F_{e}^{\prime}\left(x-x_{e}\right)+\frac{2}{3} W_{e}^{-1 / 3} W_{e}^{\prime}\left(x-x_{e}\right)\right) * \\
\left\{\tan \delta-\tan \delta_{1}-\frac{\tan \delta_{2}-\tan \delta_{1}}{v\left(\frac{F_{l} Q}{W_{e}}\right)^{2 / 3} \frac{1}{1-\gamma}+1}-\frac{\left(\tan \delta_{2}-\tan \delta_{1}\right) v\left(F_{l} Q\right)^{2 / 3}\left(x-x_{e}\right)}{\left(v\left(\frac{F_{l} Q}{W_{e}}\right)^{2 / 3} \frac{1}{(1-\gamma)}+1\right)^{2}} *\right. \\
\left.\left(\frac{2}{3} \frac{W_{e}^{\prime}}{W_{e}^{5 / 3}(1-\gamma)}+\frac{2}{3} \frac{F_{e}^{\prime}}{W_{e}^{2 / 3}(1-\gamma)}+\frac{F_{e}^{\prime}}{W_{e}^{2 / 3}(1-\gamma)^{2}}\right)\right\} .
\end{gathered}
$$

Finally, on substituting all the approximate expansions (2.63), (2.64) and (2.73) into the ODE (2.60) the resulting leading order $\left(O\left(x-x_{e}\right)\right)$ Taylor expansion for the ODE

$$
\left(\frac{F^{2}-1}{F}\right) \frac{d F}{d x}=\left(\frac{F^{2}+2}{2}\right) \frac{d(\ln W)}{d x}+\frac{3}{2}\left(\frac{F W}{Q F_{l}}\right)^{2 / 3}(\tan \theta-\mu(F))
$$


is

$$
\begin{gathered}
2\left(F_{e}^{\prime}\right)^{2}\left(x-x_{e}\right)=\frac{3}{2} \frac{W_{e}^{\prime}}{W_{e}}+\left(x-x_{e}\right)\left(\frac{W_{e}^{\prime}}{W_{e}} F_{e}^{\prime}-\frac{3}{2} \frac{\left(W_{e}^{\prime}\right)^{2}}{\left(W_{e}\right)^{2}}+\frac{3}{2} \frac{W_{e}^{\prime \prime}}{W_{e}}\right) \ldots \\
+\frac{3}{2}\left(\frac{1}{F_{l} Q^{2 / 3}}\right)\left(W_{e}^{2 / 3}+\frac{2}{3} W_{e}^{2 / 3} F_{e}^{\prime}\left(x-x_{e}\right)+\frac{2}{3} W_{e}^{-1 / 3} W_{e}^{\prime}\left(x-x_{e}\right)\right) * \\
\cdot\left\{\tan \theta-\tan \delta_{1}-\frac{\tan \delta_{2}-\tan \delta_{1}}{v\left(\frac{F_{l} Q}{W_{e}}\right)^{2 / 3} \frac{1}{1-\gamma}+1}-\frac{\left(\tan \delta_{2}-\tan \delta_{1}\right) \nu\left(F_{l} Q\right)^{2 / 3}\left(x-x_{e}\right)}{\left(v\left(\frac{F_{l} Q}{W_{e}}\right)^{2 / 3} \frac{1}{(1-\gamma)}+1\right)^{2}} *\right. \\
\left.\left(\frac{2}{3} \frac{W_{e}^{\prime}}{W_{e}^{5 / 3}(1-\gamma)}+\frac{2}{3} \frac{F_{e}^{\prime}}{W_{e}^{2 / 3}(1-\gamma)}+\frac{F_{e}^{\prime}}{W_{e}^{2 / 3}(1-\gamma)^{2}}\right)\right\} .
\end{gathered}
$$

As $W_{e}^{\prime}$ is the correct slope, the $O(1)$ terms satisfy the expression (2.49), implying

$$
\frac{3}{2} \frac{W_{e}^{\prime}}{W_{e}}+\frac{3}{2} \frac{1}{\left(F_{l} Q\right)^{2 / 3}} W_{e}^{2 / 3}\left(\tan \theta-\tan \delta_{1}-\frac{\tan \delta_{2}-\tan \delta_{1}}{v\left(\frac{F_{l} Q}{W_{e}}\right)^{2 / 3} \frac{1}{(1-\gamma)}+1}\right)=0 .
$$

Thus, by considering only the $O\left(x-x_{e}\right)$ terms results in a quadratic equation to be solved for $F_{e}^{\prime}$

$$
\begin{aligned}
2\left(F_{e}^{\prime}\right)^{2}= & \frac{W_{e}^{\prime}}{W_{e}} F_{e}^{\prime}+\frac{3}{2}\left(\frac{W_{e}^{\prime \prime}}{W_{e}}-\frac{\left(W_{e}^{\prime}\right)^{2}}{\left(W_{e}\right)^{2}}\right) \\
& +\frac{W_{e}^{-1 / 3} W_{e}^{\prime}}{\left(F_{l} Q\right)^{2 / 3}}\left[\tan \theta-\tan \delta_{1}-\frac{\left(\tan \delta_{2}-\tan \delta_{1}\right)}{\left.v\left(\frac{F_{l} Q}{W_{e}}\right)^{2 / 3} \frac{1}{1-\gamma}+1\right]}\right. \\
& +\left(\frac{W_{e}}{F_{l} Q}\right)^{2 / 3}\left[\tan \theta-\tan \delta_{1}-\frac{\left(\tan \delta_{2}-\tan \delta_{1}\right)}{\left.v\left(\frac{F_{l} Q}{W_{e}}\right)^{2 / 3} \frac{1}{1-\gamma}+1\right] F_{e}^{\prime}}\right. \\
& -v \frac{\left(W_{e}\right)^{2 / 3}\left(\tan \delta_{1}-\tan \delta_{2}\right)}{\left(v\left(\frac{F_{l} Q}{W_{e}}\right)^{2 / 3} \frac{1}{(1-\gamma)}+1\right)^{2}}\left[\frac{W_{e}^{-5 / 3} W_{e}^{\prime}}{(1-\gamma)}\right] \\
& -v \frac{\left(W_{e}\right)^{2 / 3}\left(\tan \delta_{1}-\tan \delta_{2}\right)}{\left(v\left(\frac{F_{l} Q}{W_{e}}\right)^{2 / 3} \frac{1}{(1-\gamma)}+1\right)^{2}}\left[\frac{1}{\left.W_{e}^{2 / 3} \frac{1}{(1-\gamma)}+\frac{3}{2} \frac{1}{(1-\gamma)^{2}} \frac{1}{W_{e}^{2 / 3}}\right] F_{e}^{\prime} .} .\right.
\end{aligned}
$$

Finally, once the slope is determined, the limit's problem (2.50) is resolved for frictional flows, as well. 


\section{C. JUMP CONDITIONS}

The jump conditions are derived by integrating both the non-dimensional depth- and width-averaged mass and momentum balance equations, from $X(t)-\delta$ to $X(t)+\delta$ and taking the limit $\delta \rightarrow 0$. Both $h$ and $u$ are discontinuous at $x=X(t)$. For simplicity, we define $X^{-}$as the limit position on the left side of the jump and $X^{+}$the limit on the right side of the jump, with the shock speed $s=-d X / d t$. Utilising these definitions, we start by integrating the continuity equation, utilising Leibniz's rule

$$
\begin{gathered}
\int_{X^{-}(t)}^{X^{+}(t)}(h W)_{t}+(h u W)_{x} d x=0, \\
\frac{d}{d t} \int_{X^{-}(t)}^{X^{+}(t)} h W d x+s[h W]_{X^{-}}^{X^{+}}+[h u W]_{X^{-}}^{X^{+}}=0, \\
\text { As } \int_{X^{-}}^{X^{+}} h W d x=0 \text { and } W^{+}=W^{-}=W \rightarrow[h(u+s)]_{X^{-}}^{X^{+}}=0, \\
\text { to obtain } \\
h^{+}\left(u^{+}+s\right)=h^{-}\left(u^{-}+s\right) .
\end{gathered}
$$

Similarly, we integrate the momentum balance equations

$$
\int_{X^{-}(t)}^{X^{+}(t)}\left((h u W)_{t}+\left(h u^{2} W\right)_{x}+\frac{W}{F_{l}^{2}}\left(\frac{h^{2}}{2}\right)_{x}-\frac{1}{F_{l}^{2}} h W(\tan \theta-\mu(F))\right) d x=0 .
$$

As $W^{+}=W^{-}=W$, the above equation can be simplified into

$$
\int_{X^{-}(t)}^{X^{+}(t)}\left((h u)_{t}+\left(h u^{2}\right)_{x}+\frac{1}{F_{l}^{2}}\left(\frac{h^{2}}{2}\right)_{x}-\frac{1}{F_{l}^{2}} h(\tan \theta-\mu(F))\right) d x=0 .
$$

Utilising Leibniz's rule, $\int_{X^{-}(t)}^{X^{+}(t)} h u d x=0$ and $\int_{X^{-}(t)}^{X^{+}(t)} h(\tan \theta-\mu(F)) d x=0$, yields

$$
s[h u]_{X^{-}}^{X^{+}}+\left[h u^{2}\right]_{X^{-}}^{X^{+}}+\frac{1}{2 F_{l}^{2}}\left[h^{2}\right]_{X^{-}}^{X^{+}}=0
$$

After some manipulations, the above equation can be restated as

$$
h^{+}\left(\left(u^{+}\right)^{2}+u^{+} s\right)-h^{-}\left(\left(u^{-}\right)^{2}+u^{-} s\right)+\frac{1}{2 F_{l}^{2}}\left(\left(h^{+}\right)^{2}-\left(h^{-}\right)^{2}\right)=0,
$$

which is rewritten as

$$
\begin{gathered}
h^{+}\left(\left(u^{+}\right)^{2}+2 u^{+} s+s^{2}-u^{+} s-s^{2}\right) \\
-h^{-}\left(\left(u^{-}\right)^{2}+2 u^{-} s+s^{2}-u^{-} s-s^{2}\right)+\frac{1}{2 F_{l}^{2}}\left(\left(h^{+}\right)^{2}-\left(h^{-}\right)^{2}\right)=0 .
\end{gathered}
$$


Using the jump condition obtained from the continuity equation (2.78), equation (2.79) is restated using the following steps,

$$
\begin{gathered}
h^{+}\left(u^{+}+s\right)^{2}-h^{-}\left(u^{-}+s\right)^{2}+\frac{1}{2 F_{l}^{2}}\left(\left(h^{+}\right)^{2}-\left(h^{-}\right)^{2}\right)=0, \\
h^{+}\left(u^{+}+s\right)^{2}-h^{-}\left(\frac{h^{+}}{h^{-}}\right)^{2}\left(u^{+}+s\right)^{2}+\frac{1}{2 F_{l}^{2}}\left(\left(h^{+}\right)^{2}-\left(h^{-}\right)^{2}\right)=0, \\
\left(u^{+}+s\right)^{2} h^{+} \frac{\left(h^{-}-h^{+}\right)}{h^{-}}=\frac{1}{2 F_{l}^{2}}\left(\left(h^{-}\right)^{2}-\left(h^{+}\right)^{2}\right), \\
\left(u^{+}+s\right)^{2}=\frac{1}{2 F_{l}^{2}}\left(1+\frac{h^{-}}{h^{+}}\right) h^{-} .
\end{gathered}
$$

\section{REFERENCES}

[1] D. D. Houghton and A. Kasahara, Nonlinear shallow fluid flow over an isolated ridge, Communications on Pure and Applied Mathematics 21, 1 (1968).

[2] O. Pouliquen and Y. Forterre, Friction law for dense granular flows: application to the motion of a mass down a rough inclined plane, Journal of Fluid Mechanics 453, 133 (2002). 


\section{3}

\section{BULBOUS HEAD FORMATION IN}

\section{BIDISPERSE SHALLOW GRANULAR FLOW OVER AN INCLINED PLANE}

Rapid shallow granular flows over inclined planes are often seen in nature in the form of avalanches, landslides and pyroclastic flows. In these situations the flow develops an inversely graded (large at the top) particle-size distribution perpendicular to the plane. As the surface velocity of such flows is larger than the mean velocity, the larger material is transported to the flow front. This causes size-segregation in the downstream direction, resulting in a flow front composed of large particles. Since the large particles are often more frictional than the smalls, the mobility of the flow front is reduced, resulting in a so-called bulbous head. This study focuses on the formation and evolution of this bulbous head, which we show to emerge in both a depth-averaged continuum framework and discrete particle simulations. Furthermore, our numerical solutions of the continuum model converge to a travelling wave solution, which allows for a very efficient computation of the long-time behaviour of the flow. We use small-scale periodic discrete particle simulations to calibrate (close) our continuum framework, and validate the simple $1 D$ model with full-scale $3 D$ discrete particle simulations. The comparison shows that there are conditions under which the model works surprisingly well given the strong approximations made; for example, instantaneous vertical segregation.

\subsection{INTRODUCTION}

For accurate zonation and risk assessment, it is important to accurately predict the distance to which hazardous particulate natural flows such as debris flows, pyroclastic flows or snow slab avalanches might travel [2]. These flows are heavily influenced not only by the basal topography, but also by the mixture properties, such as water saturation

This chapter has been published in Journal of Fluid Mechanics 866, 263-297 (2019) [1]. 
and the size-distribution of the particulate components (e.g. snow, pumice, rock, sand). Large-scale experiments performed by Iverson et al. [3] showed that segregation effects significantly increase the run-out distance of granular flows over inclined planes (chute flows). On the laboratory-scale, experiments also show that granular flows of mixtures behave differently than flows of uniform materials, exhibiting interesting fingering effects [4-6].

Size-segregation can have a very strong effect on a mixture's composition, and often leads to a nearly complete separation of the different particle phases. For granular chute flows this tends to un-mix particles of different sizes into layers, creating an inversely graded structure; the larger particles tend to segregate to the upper free surface, while the smaller particles sink to the base [7]. Although there is usually some degree of diffusive remixing [8], in the bidisperse granular chute flows we consider here, layers of pure large and pure small particles develop, with only a thin layer of mixed material in-between [9].

There are many mechanisms of size-segregation in granular flows [10-18], but kinetic sieving and squeeze expulsion are the mechanisms that are often used to describe segregation in dense granular chute flows [5, 7, 9]. In this description, void spaces open up between particles, allowing for percolation downwards. Small particles are more likely to fall into these gaps, which leads to a movement of small particles towards the base of the flow. At the same time, force imbalances squeeze all particles out of their layers towards the free-surface, which leads to an upwards movement. The combination results in a net migration of small particles to the base and large particles towards the free-surface. More recently, alternative mechanisms for size-segregation, in dense chute flows, have been proposed, based on e.g. buoyancy effects [19], differences in fluctuating kinetic energy [20,21] and kinetic theory [22].

In this study, the particles not only differ in their sizes, but also in their microscopic friction coefficients; larger particles are 'rougher' than smaller particles, which is expressed as a larger microscopic-friction coefficient. This difference leads to a larger macroscopic friction-coefficient for flows consisting of larger particles than for smaller particles. This is consistent with large, geophysical particulate flows, where the smaller particles usually have a higher flowability than larger particles [23]. It also induces segregation based on microscopic friction, where smoother particles tend towards the bottom of the flow $[24,25]$. Thus the larger, rougher particles rise towards the free surface, while the smaller, smoother particles percolate towards the bottom of the flow.

The large rough particles in the higher layers of the flow are transported towards the front, due to the higher velocity near the free-surface. This creates an additional downstream ('horizontal') segregation-structure, with the front of the flow consisting of mostly large particles. Larger particles have a larger macroscopic friction coefficient than smaller particles for the same (dimensional) flow height [26-28]; this causes a reduced mobility of the flow in the large particle front compared to the bidisperse tail. In our case, this effect is even larger because, as stated before, we also use a higher microscopic friction for the large particles, compared to the small particles. This configuration, with larger frictional resistive grains at the front and finer more mobile material behind, can be unstable and leads to a lateral flow instability, commonly called the fingering instability [4]. This instability leads to the formation of levees, that channelise the flow, and therefore can have a significant influence on the run-out distance [29]. When 


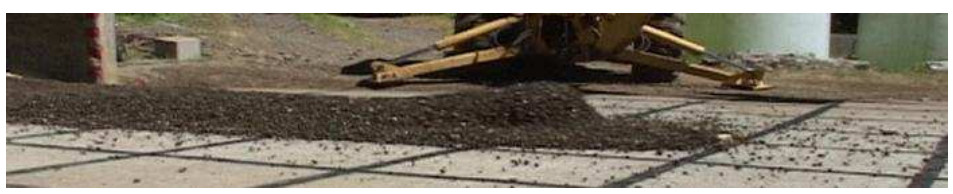

Figure 3.1: Experimental debris flow deposit at the USGS flow flume, Oregon, USA, August 2008 [34]. The back of the flow has a constant height, while the front shows evidence of a bulbous head; the flow is higher near the front than at the back of the flow. Picture courtesy of USGS.

the slope is steep enough that the flow of large particles does not arrest, the downstream segregation-structure leads to an accumulation of material in the front, leading to the so-called bulbous head, see figure 3.1. This bulbous head is seen both in geological deposits [30,31] and large-scale experiments $[3,32,33]$. The difference in friction in the front of these flows is not only due to segregation effects, but also due to differences in water-saturation: all experimental flows mentioned above are partially water-saturated, for which it is known that the front contains less water than the tail [31].

The characterisation of the behaviour of water-saturated granular flows is a topic of ongoing study. Some early experimental studies were done by Davies [35], who focused on the characterisation of granular 'waves' on moving belts, and Iverson and LaHusen [36], who studied the main origin of the frictional behaviour of water-saturated granular flows on large-scale inclined planes. They were followed by many publications, see e.g. Haas et al. [33] and references therein. At the same time, many models for (partially) water-saturated monodisperse granular flows were developed. Examples include the early model of Iverson [29], who developed a depth-averaged model based on mixture theory, and the model of [37], which uses dense kinetic theory for the granular phase. For a recent overview, we refer the interested reader to Delannay et al. [38]. To fully understand large particulate geophysical flows, it is important to understand both the influence of segregation and the influence of water-saturation in these flows; however, here we are interested in a leading-order model that does not explicitly take into account the presence of water. In section 3.3 we show that even with this strong simplification, we are able to capture many of the dynamics of these flows.

Generally, the debris-flow models discussed above do not take into account segregation effects inside granular chute flows; however, particles in such flows typically have a size-distribution that spans many orders of magnitude and will therefore segregate into layers of different sizes [39]. Here, we will investigate the influence of particle segregation on dry granular flows. We focus on bidisperse granular materials, i.e. materials consisting of just two constituents of different sizes. This greatly simplifies the problem, while maintaining the leading-order segregation behaviour $[4,40,41]$. This is a good model system to investigate the fundamental dynamics in the more complex wet geophysical problem.

Discrete particle simulations, which model the motion of each grain, are a logical choice for studying and understanding particulate flows [42, 43]. However, large geophysical flows often consist of several cubic kilometres of material; combined with the average grain-size of less than a cubic centimetre [39], this means that there can be of the order $O\left(10^{15}\right)$ particles in such a flow. Previously, discrete particle simulations have 
been shown to be very accurate for simulating segregating laboratory-scale chute flows [44]; but, are currently limited to $O\left(10^{8}\right)$ particles, which is 7 orders of magnitude less than needed for the simulation of geophysical flows.

Alternatively, to overcome the limits of computation time, one can model the flow as a continuum, expressing it in terms of the height, velocity and small/large particle concentration of the flow at any given position. Geophysical flows are often modelled using single-phase shallow layer models, that ignore effects due to segregation [45-47]. These models start with the general incompressible mass and momentum balances, which are then depth-averaged to reduce the complexity of the model. A source term is introduced to prescribe the difference between the driving force (gravitational acceleration along the slope) and the resistive force (such as basal friction).

To account for effects of segregation in granular chute flows, an advection-diffusionlike model was developed by Bridgwater et al. [48] and later Savage and Lun [9] derived an equivalent model using statistical arguments. Subsequently, models based on mixture theory have been developed, which led to the same advection-diffusion structure, e.g. [8, 20, 49-52]. For the interested reader, Tunuguntla et al. [53] gives an overview of segregation models, and uses a unified notation to compare and contrast them. Moreover, an extension to include segregation based on gradients in granular temperature has been given by Hill and Fan [54]. Note that this model reduces to the model of Gray and Chugunov [8] when kinetic stress gradients are negligible. Alternatively, one can use the more complicated models based on kinetic theory, e.g. [22, 55], which use different assumptions, for example that collisions between particles are instantaneous, binary and uncorrelated.

While the aforementioned segregation models are useful for looking at evolving segregation profiles, they can be further simplified by looking at either only the mean diameter at a certain position $(x, y)$ [56], or only some depth-averaged quantities [57]. Here, we concentrate on the depth-averaged concentration of each constituent, which requires additional assumptions on the segregation behaviour. Gray and Kokelaar [57] assumed that the flow rapidly segregates into fully separated inversely graded layers; whereas, [58] used a more complicated three layer approach. It is noteworthy that, under Gray and Kokelaar [57] assumption of instantaneous complete vertical segregation, all models compared by Tunuguntla et al. [53] reduce to the same depth-averaged segregation model that is used in this paper. Moreover, this depth-averaged model can be coupled to single-phase shallow layer models to investigate the mobility feedback that segregation provides [59]. This simple model represents the leading-order behaviour of the flow, as demonstrated by the fact that Woodhouse et al. [59] successfully showed it can reproduce the fingering instability of bidisperse granular flow fronts.

In this study, we adapt the model of Woodhouse et al. [59] by including a non-unity shape factor for the velocity profile in the momentum balance, and using a different choice for the computation of the macroscopic friction coefficient. We then show that the bulbous-head profile is also a solution of this kind of depth-averaged continuum framework when using simple initial and boundary conditions. Furthermore, we demonstrate that the bulbous head also develops in three-dimensional discrete particle simulations, with reasonable agreement between the simple one-dimensional continuum model and the fully three-dimensional discrete particle simulations. 


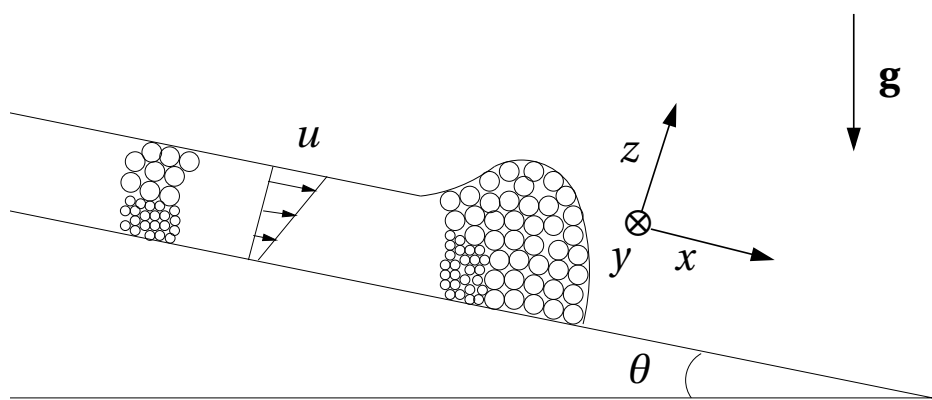

Figure 3.2: Schematic drawing of a bidisperse chute flow. The granular material flows down a chute inclined at an angle $\theta$ to the horizontal. A coordinate system is taken with the $x$-axis aligned with the downslope direction and the $z$-axis normal to the chute's surface. The flow is assumed to be uniform in the cross-slope $y$-direction. Due to the gravity, $\mathbf{g}$, pointing down, the avalanching material flows in the positive $x$-direction with a downslope velocity, $u(x, z, t)$. We assume particle-size segregation completely separates the two particle-sizes, where large particles are on top of small particles.

This paper is structured as follows: our model adapted from Woodhouse et al. [59] and our choices for the constitutive relations are discussed in section 3.2. The results of the numerical solutions of this model are described in section 3.3, where it is shown that the bulbous head emerges. Furthermore, it is shown in section 3.4 that our numerical solutions to the continuum model converge to a simple travelling wave solution, which makes computation of the long-time flow properties very efficient. Finally, in section 3.5 the results from the one-dimensional continuum model are compared to fully three-dimensional discrete particle simulations, with reasonable agreement between both approaches, despite the simplifications in the continuum model.

\subsection{ConTINUUM MODEL}

In this section, we describe the size-bidisperse shallow granular model that is used in both the time-dependent numerical solutions of section 3.3 and the travelling wave solution of section 3.4. The model is based on Woodhouse et al. [59], with some generalisations that are discussed later in this section.

\subsubsection{DEFINITIONS}

We consider a plane at an angle $\theta$ to the horizontal with the $x$-axis pointing downslope, the $y$-axis pointing across the slope and the $z$-axis being aligned with the upward pointing normal. In this coordinate system, the gravity vector, $\mathbf{g}$, has the direction $(\sin \theta, 0,-\cos \theta)$ and magnitude $g$. Particles of two different sizes are released onto the inclined plane, namely small particles and large particles. These have the same density but different radii. Where appropriate, the different sizes are denoted by superscripts $L$ and $S$ for large and small particles, respectively. A schematic overview of this geometry is given in figure 3.2 .

It is assumed that gradients and velocities in the $y$-direction are neglected. We are interested in the height, $h(x, t)$, the depth-averaged downslope velocity, $\bar{u}(x, t)$, and the depth-averaged small particle concentration, $\bar{\phi}(x, t)$, at all positions, $x$, at any time, $t>0$. 
Here, the small particle concentration, $\phi$, is defined as the volume fraction of the solid phase that consists of small particles, so the large particle concentration equals $(1-\phi)$. The depth-averaged velocity and small particle concentration can be derived from their full two-dimensional equivalents $u(x, z, t)$ and $\phi(x, z, t)$ and the flow height, $h(x, t)$, as

$$
\begin{aligned}
& \bar{u}(x, t)=\frac{1}{h(x, t)} \int_{0}^{h(x, t)} u(x, z, t) \mathrm{d} z, \\
& \bar{\phi}(x, t)=\frac{1}{h(x, t)} \int_{0}^{h(x, t)} \phi(x, z, t) \mathrm{d} z .
\end{aligned}
$$

The length-scale for the non-dimensionalisation is chosen to be the large particle diameter, instead of a typical flow-height that is usually used with this kind of model. This leads to an easier comparison with discrete particle simulations later on. All variables are non-dimensionalised by the large particle diameter, $d^{* L}$, and gravitational constant, $g$, as follows: the height and length of the flow are non-dimensionalised by $d^{* L}$, time by $\sqrt{d^{* L} / g}$ and consequently the depth-averaged velocity by $\sqrt{d^{* L} g}$. We also non-dimensionalise the particle diameter for both types of particles with $d^{* L}$, and thus $d^{S}=d^{* S} / d^{* L}$ and $d^{L}=1$, respectively. The bulk density of the flow is assumed constant, and hence it does not appear in the non-dimensionalised system of equations in section 3.2 .3 .

\subsubsection{ASSUMPTIONS}

The full two-dimensional fields for the small particle concentration and velocity of the flow are needed for the derivation of the one-dimensional depth-averaged system of equations in section 3.2.3 [59]. This reconstruction is not unique, and needs assumptions on both the segregation behaviour and the velocity profile.

Regarding the segregation behaviour, it is assumed that the flow is already segregated at the inflow boundary of the domain, where the large particles flow above the small particles. Furthermore, they are assumed to stay segregated, so there is no diffusive remixing [8]. While this is a rather strong assumption, it is fairly accurate and validated by experiments and discrete particle simulations of bidisperse chute flows, where it is shown that the segregation rate is $10-30$ times higher than the diffusive-remixing rate $[44,60]$. The combination of instantaneous segregation and the absence of diffusive remixing is equivalent to the assumption that the flow is fully segregated at all times and positions. This can be summarised as

$$
\phi(x, z, t)=\left\{\begin{array}{llr}
1 & \text { if } & 0<z<h(x, t) \bar{\phi}(x, t), \\
0 & \text { if } & h(x, t) \bar{\phi}(x, t) \leq z<h(x, t),
\end{array}\right.
$$

where $h \bar{\phi}$ can also be referred to as the height of the small particle layer.

Note, that the assumption of a fully segregated flow leaves the depth-averaged segregation equation heavily dependent on the velocity profile, i.e. on how much faster the large particles in the top layers are moving compared to the small particles in the bottom layers.

The velocity profile for bidisperse flows over a rough base is complicated and cannot be described by a simple linear or Bagnold velocity profile [61, 62]. However, for very 
shallow monodisperse flows over sufficiently rough bases, a linear profile is a good approximation [26, 63, 64], while a Bagnold velocity profile is more appropriate for thicker monodisperse flows ( $>20$ particle diameters) $[52,65]$ and monodisperse flows over smooth bases $[66,67]$. For the shallow bidisperse flows studied in this paper, we use a simplified linear velocity profile with basal slip, of the form

$$
u(z)=\alpha_{s} \bar{u}+2\left(1-\alpha_{s}\right) \bar{u} \frac{z}{h}, \quad 0 \leq \alpha_{s} \leq 1 .
$$

Here, $\alpha_{s}$ is the amount of basal slip; for $\alpha_{s}=1$, equation (3.4) describes a plug flow, while for $\alpha_{s}=0$ it describes a linear velocity profile with no slip at the base ${ }^{1}$. The choice of the constant value of $\alpha_{s}$ is discussed in section 3.2.4.

It must be noted that the linear velocity profile (3.4) is deeply embedded in the depthaveraged segregation equation (3.9), and that this equation will change drastically for different velocity profiles. For a detailed derivation of the depth-averaged segregation equation with other velocity profiles, we refer the interested reader to [6].

Using the velocity profile (3.4), we can compute the shape factor, $\alpha$, as

$$
\alpha=\frac{\overline{u^{2}}}{\bar{u}^{2}}=\frac{1}{3}\left(1-\alpha_{s}\right)^{2}+1 .
$$

This is where our model differs from that of Woodhouse et al. [59], who set $\alpha=1$ independent of $\alpha_{s}$. We choose to retain the dependency of $\alpha$ on $\alpha_{s}$ to keep the model consistent. Moreover, choosing $\alpha \neq 1$ is important to preserve the influence of inertia terms, especially for rapid flows [69]. The downside of using a non-unity shape factor is that the slope of the height at the front goes to zero, which causes numerical difficulties. Note, that the assumption $\alpha=1$ also shows the bulbous-head profile. Still, we prefer to keep the model internally consistent and hence choose $\alpha$ as given by (3.5).

\subsubsection{SYSTEM OF EQUATIONS}

With the above assumptions on the segregation behaviour and the velocity profile, we can now formulate our depth-averaged, non-dimensionalised governing equations, based on the model of Woodhouse et al. [59]. The mass- and momentum balance are respectively given by

$$
\begin{aligned}
\frac{\partial h}{\partial t}+\frac{\partial}{\partial x}(h \bar{u}) & =0 \\
\frac{\partial}{\partial t}(h \bar{u})+\frac{\partial}{\partial x}\left(\alpha h \bar{u}^{2}+\frac{1}{2} h^{2} \cos \theta\right) & =h S(h, \bar{u}, \bar{\phi}),
\end{aligned}
$$

where the source term, $S$, consists of the down-slope component of the gravitational acceleration and the basal friction respectively:

$$
S=\sin \theta-\mu(h, \bar{u}, \bar{\phi}) \frac{\bar{u}}{|\bar{u}|} \cos \theta .
$$

\footnotetext{
${ }^{1}$ Note, that $\alpha_{s}$ is often denoted by $\alpha$, see e.g. [6, 49, 59]. However, this causes a conflict in nomenclature with the shape factor, which we here denote by $\alpha$, see equation (3.5), as is used by many authors e.g. [26, 68, 69].
} 
The gravitational acceleration drives the flow in positive $x$-direction, while the basal friction opposes the avalanche motion. It can be described with a basal friction coefficient, $\mu$, which is the ratio of the shear and normal stress at the base. Traditionally, $\mu$ is taken either as a constant, e.g. [45], or as a function of the height and velocity of the flow, e.g. [70]. Here, we generalise the computation of $\mu$ to also include a dependence on the depth-averaged concentration of small particles, as discussed later in this section.

Furthermore, the depth-averaged conservation of small particle mass can be expressed as [57]

$$
\frac{\partial}{\partial t}(h \bar{\phi})+\frac{\partial}{\partial x}\left(h \bar{\phi} \bar{u}-\left(1-\alpha_{s}\right) h \bar{\phi} \bar{u}(1-\bar{\phi})\right)=0,
$$

where it must be noted that $h \bar{\phi} \bar{u}=\left(h \bar{\phi} \bar{u}-\left(1-\alpha_{s}\right) h \bar{\phi} \bar{u}(1-\bar{\phi})\right)$ when using the linear velocity profile with basal slip (3.4).

Note, that the macroscopic friction coefficient, $\mu$, is the only feedback mechanism affecting the segregation behaviour of the bulk; the depth-averaged mass and momentum balances (3.6)-(3.7) do not involve gradients in the concentration of small particles, $\bar{\phi}$. To make the macroscopic friction coefficient, $\mu$, dependent on the depth-averaged small-particle concentration, $\bar{\phi}$, we follow the approach of Pouliquen and Vallance [4]: the total basal friction is written as a concentration-weighted sum of the macroscopic friction coefficients $\mu^{S}$ and $\mu^{L}$ for mono-dispersed flows of small and large particles respectively:

$$
\mu=\bar{\phi} \mu^{S}+(1-\bar{\phi}) \mu^{L}
$$

This provides a simple way of increasing the frictional resistance to motion as the proportion of larger particles increases at the flow front (with $\mu^{L}>\mu^{S}$ ). Other friction laws are possible, for example the basal friction could be dependent on the local concentration of large and small particles at the base of the flow, or there may be a complex non-linear dependence, as shown in the discrete particle simulations of Rognon et al. [61].

For the basal friction coefficients of each pure individual constituent, we use the nondimensionalised version of the friction law for rough beds of Weinhart et al. [26], which is closely related to Forterre and Pouliquen $[68]^{2}$, and has the form

$$
\mu^{v}=\tan \delta_{1}^{v}+\frac{\tan \delta_{2}^{v}-\tan \delta_{1}^{v}}{\frac{\beta^{v} h}{A^{v} d^{v} F}+1} \quad v=S, L,
$$

where the Froude number $F=\frac{|\bar{u}|}{\sqrt{h \cos \theta}}$ can be expressed in terms of the non-dimensional velocity, $\bar{u}$, and height, $h$, of the flow, and the $z$-component of gravity, $\cos \theta$. Concerning the non-dimensional friction parameters for each constituent $v, \delta_{1}^{v}$ is the minimum chute-angle required for flow, $\delta_{2}^{v}$ is the maximum chute-angle for which steady flows are possible, $A^{v}$ is a characteristic dimensionless scale and $\beta^{v}$ is an empirical constant. The friction law (3.11) is the second point where our model differs from [59], who used an exponential version of this friction law [70]. The advantages of the reciprocal friction law

\footnotetext{
${ }^{2}$ The definition of the Froude number is different in [26] compared to [68], which results in a slightly different form of the friction law. Here, the definition of Weinhart et al. [26] is used.
} 


\begin{tabular}{llll|ll}
\multicolumn{5}{c|}{} & \multicolumn{2}{l}{ Other parameters } \\
small particles & $\begin{array}{c}\text { Friction parameters } \\
\text { large particles }\end{array}$ & & \\
\hline$\delta_{1}^{S}$ & $17.92^{\circ}$ & $\delta_{1}^{L}$ & $18.60^{\circ}$ & $\theta$ & $24^{\circ}$ \\
$\delta_{2}^{S}$ & $36.53^{\circ}$ & $\delta_{2}^{L}$ & $30.06^{\circ}$ & $\alpha_{s}$ & 0.17 \\
$A^{S}$ & 0.71 & $A^{L}$ & 3.07 & $h^{\text {in }}$ & 15 \\
$\beta^{S}$ & 0.148 & $\beta^{L}$ & 0.146 & $\bar{\phi}^{\text {in }}$ & 0.5
\end{tabular}

Table 3.1: Summary of parameters for the continuum model used in this paper. The friction parameters, $\delta_{1}, \delta_{2}, A$ and $\beta$, depend on the granular materials used, while $\theta, h^{\text {in }}$ and $\bar{\phi}^{\text {in }}$ depend on the geometry. The procedure we used for determining the friction parameters, for a given granular material, is based on the experiments of [70] and is fully detailed in [27]. The basal slip, $\alpha_{s}$, depends on both the material and the geometry.

(3.11) are that it both gives a better fit, and is computationally faster compared to the exponential friction law [26]. Moreover, it has been shown by Jop et al. [71] that the friction law (3.11) is closely related to the popular $\mu(I)$-rheology [64] evaluated at the base.

One of the major advantages of the model described in this section is that it consists entirely of non-dimensional quantities; one can use this model across many different scales, from the laboratory-scale to full particulate geophysical flows. Recently, Kokelaar et al. [72] presented some field-evidence that these models can describe large-scale features on the moon, in addition to the lab-scale phenomena they have already been shown to capture.

\subsubsection{Closure Relations}

In this section, closure relations for the macroscopic friction-coefficient, $\mu$, and the shapefactor, $\alpha$, are presented for incorporating the material-dependent properties into the model (3.6) - (3.11). To determine the friction parameters in (3.11), one can use smallscale periodic discrete particle simulations, as shown by Weinhart et al. [26] and Thornton et al. [27].

For glass beads with a volume ratio $V^{L} / V^{S}=2$ on a rough bottom of small beads, the friction parameters have been determined by [73], using the open-source software package MercuryDPM [44, 74-76].

Similarly, the shape factor, $\alpha$, can be measured from small-scale discrete particle simulations [26]. For the shallow bidisperse flows in this study, we observed a heightdependence for this shape factor, similar to the relation for monodisperse flows seen by Weinhart et al. [26]: with increasing height, $\alpha$ decreases asymptotically to a fixed value, in our case approximately 1.2. This is lower than the value of 1.25 , observed by Weinhart et al. [26], for monodisperse flows with a Bagnold velocity profile. For simplicity, we take $\alpha=\alpha\left(h^{\text {in }}, \theta\right)$ constant throughout the domain, depending only on the inflow height and the chute angle. For example, for inflow-height $h^{\text {in }}=15$ and angle $\theta=24^{\circ}$, a constant value $\alpha \approx 1.23$ is used throughout the whole domain. Combined with (3.5), this results in $\alpha_{s} \approx 0.17$ as the constant for the basal slip in the velocity profile (3.4). All relevant parameters for the continuum model used to obtain the results presented in this paper are summarised in table 3.1. 


\subsection{TIME-DEPENDENT NUMERICAL SOLUTION}

In this section, we solve the model outlined in section 3.2 numerically in order to show that it leads naturally to the bulbous head, where the flow is thicker downstream near the front than further upstream. Since the system is hyperbolic, it can be simulated with a discontinuous Galerkin finite element method (DGFEM) [77]. This type of method combines the numerical fluxes of finite volume methods with the high-order polynomial approximations through basis functions of finite element methods. DGFEMs are widely used for computational fluid dynamics, see for example Shu [78] and the references therein. One of the advantages of DGFEM over conforming FEM is that it can deal with discontinuities in the solution, without producing spurious oscillations. We follow the general DGFEM framework for a second-order scheme with the HLL flux [79], the TVB limiter of Cockburn and Shu [80] and the wetting/drying treatment of Bunya et al. [81]. The details of the numerical method used can be found in appendix 3.A. The method is implemented in the hpGEM software package [82, 83], which provides a framework for DGFEM simulations. Due to the wetting and drying treatment and the small but finite gradients of the height in the front, the accuracy of the method is reduced to first order.

A domain $x \in\left[0, x_{\text {end }}\right]$ is constructed with an appropriate choice of $x_{\text {end }}$, such that this end boundary does not influence the flow profile. The initial conditions are chosen to represent an empty chute, so $h(x, 0)=h \bar{u}(x, 0)=h \bar{\phi}(x, 0)=0$. At the inflow boundary $x=0$, Dirichlet boundary conditions are prescribed to fix the inflow values, $h(0, t)=h^{\mathrm{in}}$, $h \bar{u}(0, t)=h^{\text {in }} \bar{u}^{\text {in }}$ and $h \bar{\phi}(0, t)=h^{\text {in }} \bar{\phi}^{\text {in }}$. These inflow values are chosen such that the flow at $x=0$ is non-accelerating, i.e. $S\left(h^{\mathrm{in}}, \bar{u}^{\mathrm{in}}, \bar{\phi}^{\mathrm{in}}\right)=0$. At the outflow boundary $x=x_{\text {end }}$, outflow boundary conditions are prescribed [84]. Since the bulk flow never reaches this boundary, the exact boundary conditions do not matter, as long as they keep the height, momentum and small particle height non-negative and nearly zero.

For the results presented in this paper, a granular flow over a chute at angle $\theta=24^{\circ}$ has been simulated until $t_{\text {end }}=2500$. For this end-time, an appropriate choice for the length of the domain is $x_{\text {end }}=10000$. In order to be able to compare the DGFEM solutions with the discrete particle simulations of te Voortwis [73], the inflow height and small particle concentration are prescribed as $h^{\text {in }}=15, \bar{\phi}^{\text {in }}=0.5$. For a flow with uniform inflow height, it then follows that $\bar{u}^{\text {in }} \approx 3.4$, see appendix 3.B.1. For DGFEM simulations, we have chosen a time step of $\Delta t=10^{-4}$ and $N=250$ elements. All parameter values used in the numerical solutions are summarised in table 3.1.

The height and concentration profiles that emerge from these DGFEM solutions can be found in figure 3.3. Since the initial- and boundary- conditions are exactly the same as in a dam-break structure, the profiles are initially very similar to this structure, see figure 3.3(a) [85]. We see that the bulbous head has not formed yet, and the profile of the small particle concentration is smooth. Moreover, it is noteworthy that as a result of choosing $\alpha \neq 1$, the height tends asymptotically to zero and a thin precursor layer arises. The smooth profile with gradient continuously decaying to zero is consistent with monodisperse dam-breaks and chute flows, for which analytical solutions have been derived by Hogg and Pritchard [85] and Saingier et al. [69].

Around $t \approx 250$ the flow starts to develop a clear front of only large particles, separated by a shock in the small particle concentration, see figure 3.3(b). Furthermore, the 

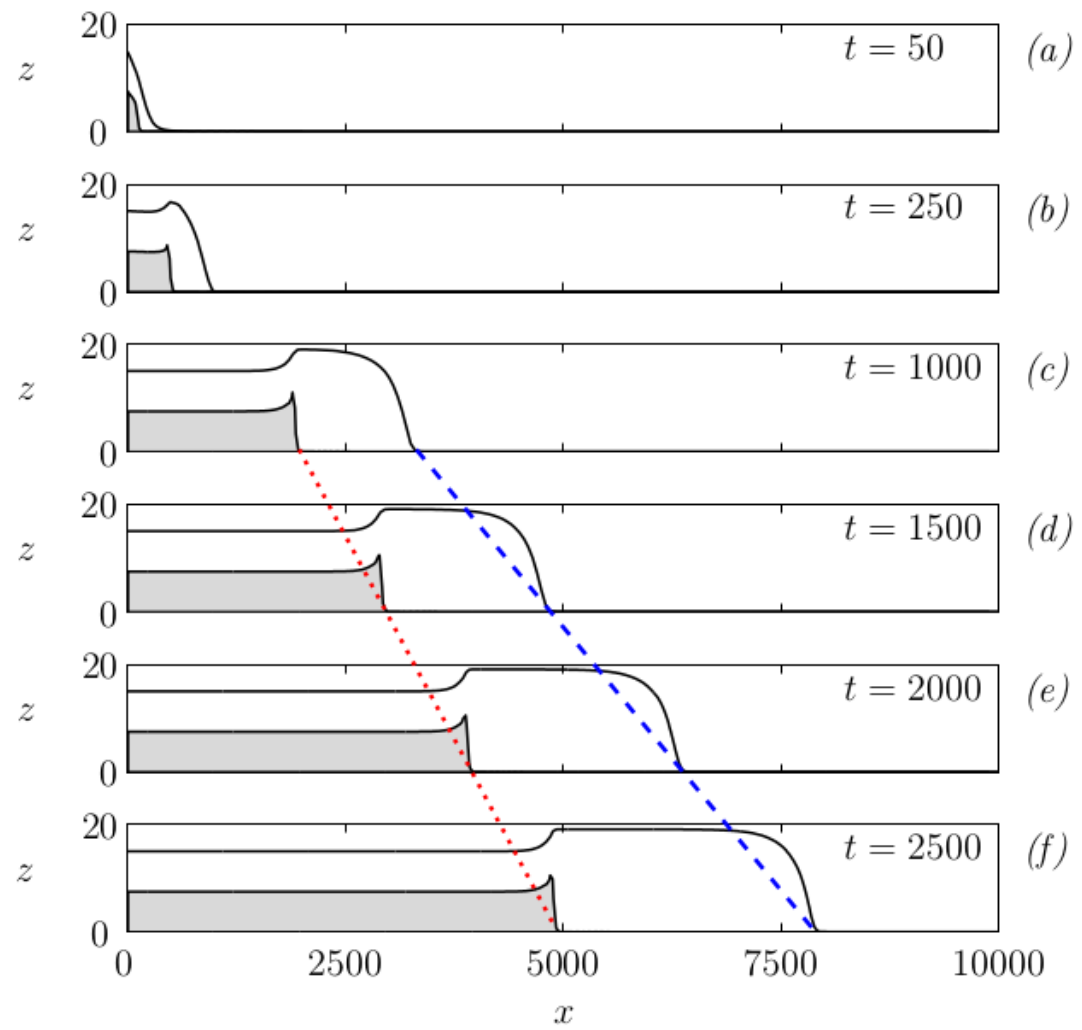

Figure 3.3: Height profiles at various times generated by DGFEM solutions. The thick black curve denotes the height, $h$, of the flow, the gray area is bounded by the height of the small particles, $h \bar{\phi}$. Both $x$ and $z$ are scaled by the large particle diameter, $d^{* L}$. Initially, the bulbous head shape develops (a), (b), until the head reaches its maximum height (c). It then advects downwards, with the faster large particle front growing longer at a constant rate. The dotted red and dashed blue lines illustrate the different speeds of the shock position and large particle front, respectively. Note, the axis has been significantly compressed in $x$-direction, in order to fit the page. 
friction causes the large particle front to have a lower speed than the inflowing material. This makes the height deviate from the dam-break structure, towards a structure in which the height at the front is higher than at the inflow. Once the head is fully developed, around $t \approx 1000$ (figure 3.3(c)), the bulbous head height stays constant. Near the inflow, the flow is uniform, with the prescribed height, velocity and small particle concentration. The bulbous head at the front is now clearly higher than the inflow height, with a very pronounced large particle front.

When comparing the profiles at $t=1000, t=1500, t=2000$ and $t=2500$ (figure 3.3(c)-(f)), we see that the shock in small particle concentration moves with a constant rate, as illustrated by the dotted red line. In the region just behind this shock, the height profile and small particle concentration profile stay constant with respect to the shock. Near the inflow, the height and small particle concentration stay constant at their prescribed values. So up to the shock position, the profiles of the height and small particle concentration essentially stay the same, they are simply advected in the $x$-direction and have a growing 'tail'. This indicates that there may be a travelling wave solution of the model we presented in section 3.2. In section 3.4 we derive such a travelling wave solution.

The large-particle front grows longer with time, and moves with a constant speed. This speed is illustrated by the dashed blue line in figure 3.3(c)-(f). The speed of the large particle front is higher than the shock-speed, which is represented by the different slopes of the dotted red and dashed blue lines in figure 3.3(c)-(f). The shape of the front stays the same, suggesting that this can be described by another travelling wave solution of this model. In section 3.4, it is shown that the large particle front indeed fits a travelling wave solution, namely the monodisperse front solution derived by Saingier et al. [69], which is a generalisation for $\alpha \neq 1$ of the solutions of Pouliquen [86] and Gray and Ancey [41].

In the next section, the travelling wave solution for the region behind the shock will be derived. Furthermore, we show that the numerical solutions of this section converge to a combination of two travelling wave solutions with different speeds, one with a shock in the small-particle concentration and one for the mono-disperse front.

\subsection{TRAVELling WAVE SOLUTION}

In this section, a travelling wave solution is derived based on the observations from the numerical solution of section 3.3. That is, we seek for a steady-solution in a reference frame moving at constant speed. So if we can capture this wave in a reference frame, there exists a frame speed, $u_{f}$, for which the travelling wave solution is a steady-state solution in this moving frame.

Based on our observations in section 3.3, we postulate a travelling wave solution to system (3.6)-(3.9) in which the profiles of $h$ and $\bar{u}$ are continuous, but there is a steady moving shock in $\bar{\phi}$. This shock speed is the same as the frame speed, $u_{f}$, as we want the shock to stand still in the moving frame. We can introduce a new coordinate system

$$
\tau=t, \quad \xi=x-u_{f} t,
$$

such that the frame moves with speed $u_{f}$ in the positive direction with $\xi=0$ being the 
fixed position of the shock. Applying this coordinate transformation leads to the system

$$
\begin{aligned}
\frac{\partial h}{\partial \tau}+\frac{\partial}{\partial \xi}\left(h\left(\bar{u}-u_{f}\right)\right) & =0, \\
\frac{\partial}{\partial \tau}(h \bar{u})+\frac{\partial}{\partial \xi}\left(h \bar{u}\left(\alpha \bar{u}-u_{f}\right)+\frac{1}{2} h^{2} \cos \theta\right) & =h S(h, \bar{u}, \bar{\phi}), \\
\frac{\partial}{\partial \tau}(h \bar{\phi})+\frac{\partial}{\partial \xi}\left(h \bar{\phi}\left(\bar{u}-u_{f}\right)-h \bar{\phi} \bar{u}\left(1-\alpha_{s}\right)(1-\bar{\phi})\right) & =0 .
\end{aligned}
$$

Note, that the source term, $S$, given by (3.8) remains unchanged by this transformation since it does not directly depend on $x$ or $t$.

As we are interested in a travelling wave solution, we assume that the solution of the system is in steady-state with respect to the new coordinate system. Therefore, we set the $\tau$-derivatives to zero, so the system becomes a set of coupled ordinary differential equations:

$$
\begin{aligned}
\frac{d}{d \xi}\left(h\left(\bar{u}-u_{f}\right)\right) & =0, \\
\frac{d}{d \xi}\left(h \bar{u}\left(\alpha \bar{u}-u_{f}\right)+\frac{1}{2} h^{2} \cos \theta\right) & =h S(h, \bar{u}, \bar{\phi}), \\
\frac{d}{d \xi}\left(h \bar{\phi}\left(\bar{u}-u_{f}\right)-h \bar{\phi} \bar{u}\left(1-\alpha_{s}\right)(1-\bar{\phi})\right) & =0 .
\end{aligned}
$$

Equations (3.16) and (3.18) can be integrated directly:

$$
\begin{aligned}
h\left(\bar{u}-u_{f}\right) & =C_{1}, \\
h \bar{\phi}\left(\bar{u}-u_{f}\right)-h \bar{\phi} \bar{u}\left(1-\alpha_{s}\right)(1-\bar{\phi}) & =C_{2},
\end{aligned}
$$

where $C_{1}$ and $C_{2}$ are arbitrary integration constants. Based on the numerical results in section 3.3, we assume that the height, $h$, momentum, $h \bar{u}$, and small particle height, $h \bar{\phi}$, go asymptotically to a fixed value for $\xi \rightarrow-\infty$, so $h \rightarrow h^{\text {in }}, \bar{u} \rightarrow \bar{u}^{\text {in }}$ and $\bar{\phi} \rightarrow \bar{\phi}^{\text {in }}$ as $\xi \rightarrow-\infty$ for some constants $h^{\text {in }}>0, \bar{u}^{\text {in }}>0$ and $\bar{\phi}^{\text {in }} \in[0,1]$. Using (3.19) and (3.20), the integration constants, $C_{1}$ and $C_{2}$, are then given by

$$
\begin{aligned}
& C_{1}=h^{\text {in }}\left(\bar{u}^{\text {in }}-u_{f}\right), \\
& C_{2}=h^{\text {in }} \bar{\phi}^{\text {in }}\left(\bar{u}^{\text {in }}-u_{f}\right)-h^{\text {in }} \bar{\phi}^{\text {in }} \bar{u}^{\text {in }}\left(1-\alpha_{s}\right)\left(1-\bar{\phi}^{\text {in }}\right) .
\end{aligned}
$$

Substituting (3.21) into (3.19) gives the relation for the height depending on the depthaveraged velocity

$$
h(\bar{u})=\frac{h^{\text {in }}\left(\bar{u}^{\text {in }}-u_{f}\right)}{\bar{u}-u_{f}} .
$$

Similarly, substitution of (3.22) into (3.20) and rearrangement of the terms shows that $\bar{\phi}$ is a solution to the quadratic equation

$$
\left(1-\alpha_{s}\right) \bar{u} \bar{\phi}^{2}+\left(\alpha_{s} \bar{u}-u_{f}\right) \bar{\phi}-\frac{C_{2}}{h}=0 .
$$


This equation can be solved with the quadratic formula, so if $\bar{u}$ is known, then $h(\bar{u})$ follows from (3.23), and $\bar{\phi}(\bar{u})$ can be found from

$$
\bar{\phi}(\bar{u})=\frac{-\left(\alpha_{s} \bar{u}-u_{f}\right)+\sqrt{\left(\alpha_{s} \bar{u}-u_{f}\right)^{2}+4\left(1-\alpha_{s}\right) \bar{u} C_{2} / h(\bar{u})}}{2\left(1-\alpha_{s}\right) \bar{u}} .
$$

Note, that the other solution to the quadratic equation (3.24) gives a negative value for $\bar{\phi}$, which is unphysical. Using these results to eliminate $h$ and $\bar{\phi}$ from (3.17) leads to a first order nonlinear ordinary differential equation for $\bar{u}$ :

$$
\left(2 \alpha \bar{u}-u_{f}-\frac{\bar{u}\left(\alpha \bar{u}-u_{f}\right)}{\bar{u}-u_{f}}-\frac{h^{\mathrm{in}}\left(\bar{u}^{\mathrm{in}}-u_{f}\right) \cos \theta}{\left(\bar{u}-u_{f}\right)^{2}}\right) \frac{d \bar{u}}{d \xi}=S(h(\bar{u}), \bar{u}, \bar{\phi}(\bar{u})) .
$$

\subsubsection{BOUNDARY CONDITIONS}

As in section 3.3, we prescribe the inflow height, $h^{\text {in }}$, and the inflow concentration of small particles, $\bar{\phi}^{\text {in }}$. We also want the height to go to the inflow height asymptotically for $\xi \rightarrow-\infty$, so $\left(\frac{d h}{d \xi}\right)^{\text {in }}=0$ is prescribed. Using equation (3.16) and the chain rule, it follows that

$$
\left(\frac{d \bar{u}}{d \xi}\right)^{\text {in }}=\frac{-C_{1}}{\left(h^{\text {in }}\right)^{2}}\left(\frac{d h}{d \xi}\right)^{\text {in }}=0 .
$$

Equations (3.26) and (3.27) now imply that $\bar{u}^{\text {in }}$ must satisfy $S\left(h^{\text {in }}, \bar{u}^{\text {in }}, \bar{\phi}^{\text {in }}\right)=0$. For the current friction law (3.10)-(3.11), this is given by:

$$
\bar{u}^{\text {in }}=f\left(\bar{\phi}^{\text {in }}\right)\left(h^{\text {in }}\right)^{3 / 2},
$$

where $f\left(\bar{\phi}^{\mathrm{in}}\right)$ is a function of $\bar{\phi}^{\mathrm{in}}$, the friction parameters and the geometry. For the exact form, see appendix 3.B.1.

Furthermore, it is beneficial to know the values $h^{\text {out }}$ and $\bar{u}^{\text {out }}$ at the outflow position of the domain, $\xi \rightarrow \infty$. We look for a flow that is steady for $\xi>0$, with no small particles in this region, so $\bar{\phi}=0$ if $\xi>0$. To determine the values of $h^{\text {out }}$ and $\bar{u}^{\text {out }}$, we use the global mass balance,

$$
h^{\text {in }}\left(\bar{u}^{\text {in }}-u_{f}\right)=h^{\text {out }}\left(\bar{u}^{\text {out }}-u_{f}\right) .
$$

Furthermore, we prescribe that the flow is non-accelerating and uniform with only large particles in the front:

$$
S\left(h^{\text {out }}, \bar{u}^{\text {out }}, 0\right)=0 \text {. }
$$

Note, that conditions (3.28) and (3.30) only hold for steady flows; in particular, in the initial phase of the flows described in section 3.3, these conditions do not hold. Conditions (3.29) and (3.30) imply that the height and depth-averaged velocity have constant values $h=h^{\text {out }}$ and $\bar{u}=\bar{u}^{\text {out }}$ for $\xi>0$. They can be computed with Matlab's solve routine from

$$
\left(\bar{u}^{\text {out }}\right)^{5 / 3}-u_{f}\left(\bar{u}^{\text {out }}\right)^{2 / 3}-\left(\frac{\tan \theta-\tan \delta_{1}^{L}}{\tan \delta_{2}^{L}-\tan \theta} \frac{\beta^{L} \sqrt{\cos \theta}}{A^{L}}\right)^{2 / 3} h^{\text {in }}\left(\bar{u}^{\text {in }}-u_{f}\right)=0 .
$$

The value of $h^{\text {out }}$ then follows directly from (3.29). 


\subsubsection{SHOCK PROPERTIES}

To compute the frame speed, $u_{f}$, consider a plane perpendicular to the chute at a certain $\xi=\xi_{0}<0$. Since we are looking at a steady state solution in the moving frame and there are no small particles at the front, we know that the net volume flux of small particles through this plane is zero. Because we assume that the flow is fully segregated, this can be expressed as

$$
\int_{0}^{\bar{\phi} h}\left(u(z)-u_{f}\right) d z=0
$$

Evaluating this integral at $\xi \rightarrow-\infty$ with the linear velocity profile (3.4), it can be shown that the shock speed is given by

$$
u_{f}=\bar{u}^{\mathrm{in}}\left(\alpha_{s}+\left(1-\alpha_{s}\right) \bar{\phi}^{\mathrm{in}}\right) .
$$

To compute the small particle concentration at the left side of the shock, we realise that for a general hyperbolic system of partial differential equations of the form $\partial u / \partial t+$ $\partial f(u) / \partial x=0$, the Rankine-Hugoniot jump condition states that the shock speed $s$ satisfies $s\left(u^{+}-u^{-}\right)=f\left(u^{+}\right)-f\left(u^{-}\right)$[87]. Applying this to the relation for the small particle concentration (3.9) and shock speed equal to the frame speed, $u_{f}$, we find

$$
\begin{aligned}
u_{f}\left((h \bar{\phi})^{+}-(h \bar{\phi})^{-}\right)= & \left((h \bar{\phi})^{+}(\bar{u})^{+}-(h \bar{\phi})^{+}(\bar{u})^{+}\left(1-\alpha_{s}\right)\left(1-(\bar{\phi})^{+}\right)\right) \\
& -\left((h \bar{\phi})^{-}(\bar{u})^{-}-(h \bar{\phi})^{-}(\bar{u})^{-}\left(1-\alpha_{s}\right)\left(1-(\bar{\phi})^{-}\right)\right),
\end{aligned}
$$

where $(\bar{\phi})^{-}=\lim _{\xi \uparrow 0} \bar{\phi}$ and $(\bar{\phi})^{+}=\lim _{\xi \downarrow 0} \bar{\phi}$. Since $h$ and $\bar{u}$ are continuous at the shock position, it holds that $(h)^{-}=(h)^{+}=h^{\text {out }}$ and $(\bar{u})^{-}=(\bar{u})^{+}=\bar{u}^{\text {out }}$. Working out the parentheses and substituting these relations for the height and depth-averaged velocity in (3.34) gives

$$
u_{f}=\alpha_{s} \bar{u}+\bar{u}\left(1-\alpha_{s}\right)\left((\bar{\phi})^{+}+(\bar{\phi})^{-}\right) .
$$

Assuming the small particle concentration at the downstream position of the shock disappear, $(\bar{\phi})^{+}=0$, it follows from (3.35) that

$$
\bar{\phi}^{\text {out }}=(\bar{\phi})^{-}=\frac{u_{f}-\alpha_{s} \bar{u}^{\text {out }}}{\left(1-\alpha_{s}\right) \bar{u}^{\text {out }}},
$$

is the value of the small particle concentration at the left side of the shock.

\subsubsection{ODE SOLUTION}

The last missing step from the travelling wave solution is the exact shape of the height, velocity, and concentration profiles. In order to compute these profiles, equation (3.26) is solved with Matlab's ode 45 routine using the parameters of table 3.1. The boundary conditions at the shock position, $\xi=0$, are $h=h^{\text {out }}, \bar{u}=\bar{u}^{\text {out }}$ and $(\bar{\phi})^{-}=\bar{\phi}^{\text {out }}$ and $(\bar{\phi})^{+}=$ 0 . Equation (3.26) is integrated in both positive and negative $\xi$-direction starting from $\xi=0$ with the different initial conditions for $\bar{\phi}$.

The resulting profiles are displayed in figure 3.4. For $\xi>0$, the flow is non-accelerating, since the height, velocity and small particle concentration stay constant, in accordance 

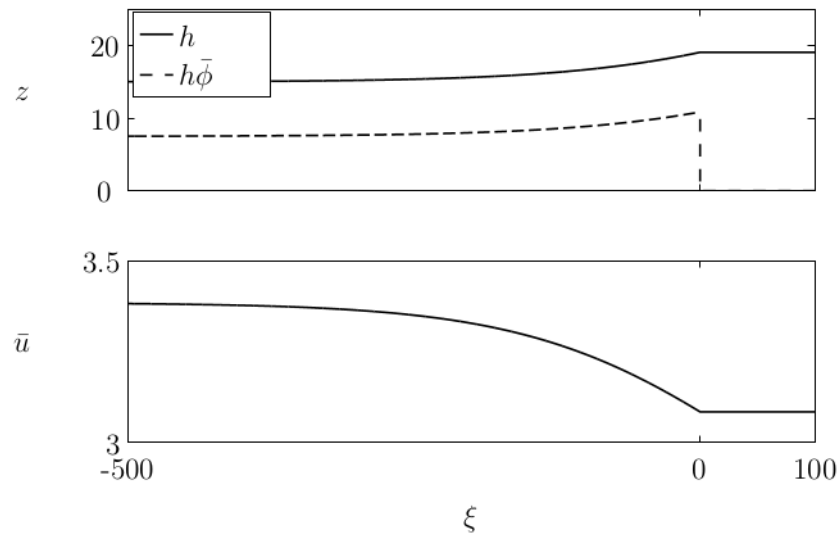

Figure 3.4: Profiles for the travelling wave solution for the height $h$, height of small particles $h \bar{\phi}$ and depthaveraged velocity $\bar{u}$ of the flow. Note, that this solution is valid for $\xi \in(-\infty, \infty)$, with $h \rightarrow h^{\text {in }}, \bar{\phi} \rightarrow \bar{\phi}^{\text {in }}$ and $\bar{u} \rightarrow \bar{u}^{\text {in }}$ as $\xi \rightarrow-\infty$ and $h=h^{\text {out }}, \bar{\phi}=0$ and $\bar{u}=\bar{u}^{\text {out }}$ for $\xi>0$. Therefore the total travelling wave solution has infinite mass.

with constraint (3.30). There are no small particles present, since $\bar{\phi}=0$ for all $\xi>0$. The flow for $\xi<0$ is more interesting, where it is important to note that $h \rightarrow h^{\mathrm{in}}, \bar{u} \rightarrow \bar{u}^{\mathrm{in}}$ and $\bar{\phi} \rightarrow \bar{\phi}^{\text {in }}$ asymptotically as $\xi \rightarrow-\infty$. All curves are smooth, except for the shock in $\bar{\phi}$ at $\xi=0$.

Comparing the inflow and outflow heights and velocities, we see that the flow in the front is deeper and slower than the flow in the back. This is caused by the higher friction in the front, since there are only large, more frictional, particles here. The higher friction coefficient causes more momentum to be dissipated, but the mass must be conserved; therefore, the flow of pure large particles must be deeper to conserve mass. Of course, the magnitude of these differences depend on the difference in friction of both constituents. The larger the difference in friction between the bidisperse and large particle phases, the deeper and slower the flow in the front. This influence was verified by changing various combinations of friction parameters in (3.26), e.g. by making $A, \delta_{1}^{L}$ and $\delta_{2}^{L}$ larger or smaller. The exact relation between the macroscopic friction coefficients and flow-height for a bidisperse granular flow within the bulbous head needs further investigation. However, a detailed investigation of this relationship, for the similar constant height breaking wave-structure can be found in van der Vaart et al. [88]

The shock speed of $u_{f} \approx 1.9$ is significantly lower than the outflow speed, $\bar{u}^{\text {out }} \approx 3.1$, which might look surprising at first. However, we assume that the velocity of the flow at the surface is higher than at the base, varying linearly as described by (3.4). The small particles are at the base, and therefore moving much slower than the large particles at the free-surface. Thus the shock in the small particle concentration must be located at the base of the flow, since that is where all small particles reside. It follows that the shock speed must be the same as the average velocity of the flow from the base to the small 
particle height, which can be expressed as

$$
\begin{aligned}
u_{f} & =\alpha_{s} \bar{u}^{\text {out }}+2\left(1-\alpha_{s}\right) \frac{\bar{u}^{\text {out }}}{h^{\text {out }}}\left(\frac{h^{\text {out }} \bar{\phi}^{\text {out }}}{2}\right), \\
& =\alpha_{s} \bar{u}^{\text {out }}+\left(1-\alpha_{s}\right) \bar{u}^{\text {out }} \bar{\phi}^{\text {out }} .
\end{aligned}
$$

This is exactly the shock speed that we see, which is significantly lower than the outflow speed. This difference in $u_{f}$ and $\bar{u}^{\text {out }}$ leads to a net transport of large particles to the front, causing the bulbous head to grow in volume. Note, that the net transport of large particles towards the front also directly follows from the travelling wave solution, see appendix 3.B.2 for the exact expression.

Next, this travelling wave solution is compared with the time-dependent DGFEM solution of section 3.3.

\subsubsection{COMPARISON WITH TIME-DEPENDENT SOLUTION}

To verify that this travelling wave solution describes the time-dependent solution of section 3.3, both solutions are compared by shifting the DGFEM solution such that the shock in small particle concentration is located at $\xi=0$ for all times, see figure 3.5. Moreover, the monodisperse front is compared to the solution of Saingier et al. [69], which satisfies the ordinary differential equation

$$
\left((\alpha-1) \frac{\left(\bar{u}^{\text {out }}\right)^{2}}{h \cos \theta}+1\right) \frac{d h}{d \tilde{x}}=\tan \theta-\mu^{L}\left(h, \bar{u}^{\text {out }}\right),
$$

where $\tilde{x}$ is the distance from the point where the height vanishes. This is a generalisation for $\alpha \neq 1$ of the monodisperse front solutions derived by Pouliquen [86] and Gray and Ancey [41]. By shifting $\tilde{x}$, we can fix the mass such that the front solution agrees with the DGFEM solutions.

Figure 3.5 shows the comparison between the DGFEM solution at $t=2500$ and both ODE solutions for the height and height of small particles. We see similar agreement for the depth-averaged velocity of the flow. For $\xi<0$, the time-dependent solution matches the travelling wave solution of this section. For $\xi>0$, the time-dependent solution matches the solution of ODE (3.39). It should be noted that both ODE solutions travel with different velocities: the large particle front travels with a speed $\bar{u}^{\text {out }}$, which is larger than the shock speed, $u_{f}$, leading to an increasing volume in the monodisperse head. Furthermore, the volume flow rate of large particles is identical for the travelling wave solution and the DGFEM solution, see appendix 3.B.2. We therefore conclude that the DGFEM solution can be seen as a combination of two travelling wave ODE solutions, travelling at different velocities. In the next section we validate the model by comparing the DGFEM solution to discrete particle simulations.

\subsection{COMPARISON WITH DISCRETE PARTICLE SIMULATIONS}

To analyse the quality of the model described in section 3.2 for this kind of flow, the DGFEM solutions are compared with two preliminary sets of discrete particle simulations of a chute flow. The discrete particle simulations are performed using MercuryDPM [75? ? ], where the simulation details can be found in appendix 3.C. The chute has 


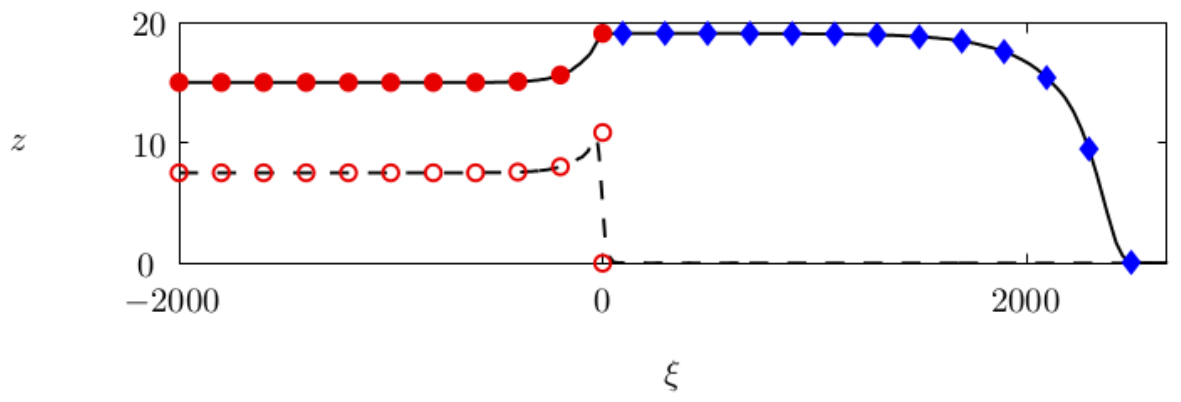

Figure 3.5: Numerical height and concentration profiles at $t=2500$ obtained from DGFEM solutions (black lines) compared with the travelling wave solution of figure 3.4 (red circles) and monodisperse front solution of (3.39) (blue diamonds). The solid lines and objects denote the height, $h$, the dotted line and open circles denote the small particle height, $h \bar{\phi}$. The profiles obtained from the DGFEM solutions are shifted in $x$-direction such that the shock is located at $\xi=0$.

the same inclination as in the DGFEM solutions and the particles are the same as used for the calibration of the friction parameters. To model the inflow boundary conditions, a so-called maser boundary has been developed: for $x \in[-20,0]$, a small periodic chute is simulated. Once the flow is fully developed, the periodic boundary is removed, and all particles flowing through the boundary at $x=0$ are both entering the non-periodic part of the chute $(x>0)$ and duplicated at the beginning of the domain, $x \approx-20$. This way, the flow has a fully developed, steady velocity- and segregation- profile at the inflow. See appendix 3.C.5 for full details of the maser inflow boundary.

\subsubsection{HEIGHT PROFILES}

Figure 3.6 shows a time-evolving height profile from our initial discrete particle simulations. Initially, at $t=0$, we only have the maser particles between $x=-20$ and $x=0$, otherwise the chute is empty. By $t=100$, the flow has already developed a front of purely large particles, that is higher than the inflow height; a bulbous head is forming. As time progresses ( $t=200$ until $t=500$ ), the bulbous head gets longer and higher, eventually saturating in height but always growing in length.

In figure 3.6, the discrete particle simulations are overlayed with the DGFEM solution at the same time. Note, that there is no fitting involved, since the friction parameters and shape factor were calibrated, i.e. measured with small-scale periodic discrete particle simulations using the same particle properties. The procedure we used for calibrating the friction parameters, for a given granular material, is based on the experiments of [70] and is fully detailed in [27]. Since the discrete particle simulations and continuum model of section 3.2 both use the large particle diameter and gravity for scaling, we can directly compare the results of both methods. Note, that the $x$-axis start at $x=-20$ to show the maser particles.

The similarity between the predicted shape of the flow-front and the discrete particle data is remarkably good, especially for larger $t$. Initially there are some discrepancies: the head is already forming at $t=100$ in the discrete particle simulations and not in the 

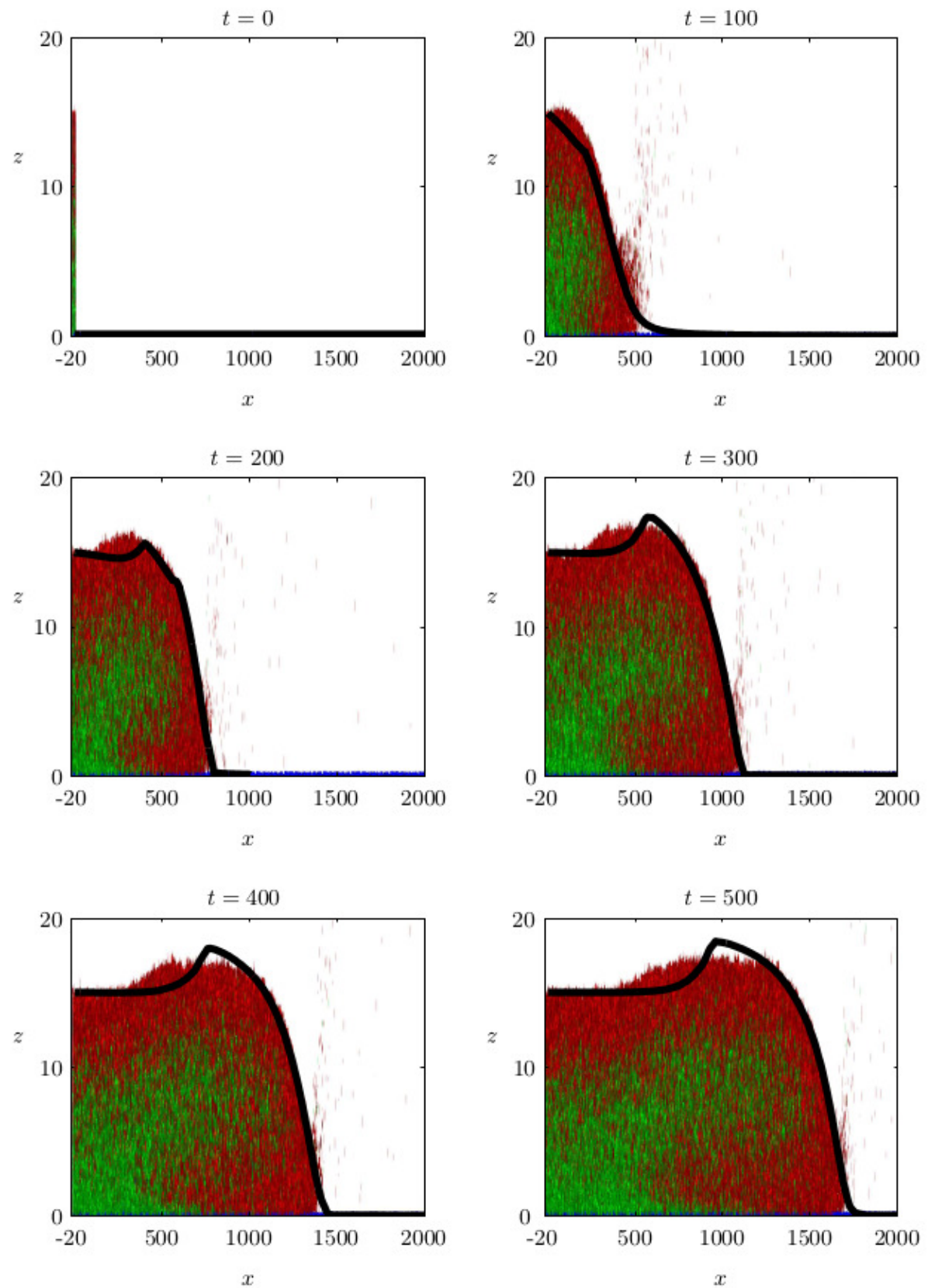

Figure 3.6: Snapshots of discrete particle simulation of a bidisperse chute flow over a rough bottom at various times. The inflow height is 15 particles diameters, resulting in supercritical inflow and the mixture is 50/50 large and small particles by volume. Red are the large particles, green are the small particles. The black lines denote the DGFEM solution for the height. Both $x$ and $z$ are scaled by the large particle diameter $d^{* L}$, in both the discrete particle simulations and DGFEM solutions. Note, that the $x$-axis is squeezed by a factor 100 compared to the $z$-axis, so the individual particles appear as very thin vertical stripes in this plot. 
continuum model. We hypothesise that this is because the DFGEM prescribed inflow conditions do not exactly match the outflow conditions of the maser, in particular, the effective friction between the flow and surface. The travelling distance of the flow is very well predicted for all $t$, even though the only condition that is prescribed in both simulation methods is that the flow is steady and non-accelerating at the inflow.

Still, the depth-averaged continuum theory does not fully capture all of the details of the height-profile. There are two main differences between the shape of the head in the continuum model and discrete particle simulations. The first is at the very front of the flow, which in the discrete particle simulations shows a gaseous behaviour with a few saltating particles being ejected from the flow. This gaseous behaviour cannot be predicted by the continuum model, since a constant bulk density is assumed. However, the main discrepancy for the height profile between the continuum model and the discrete particle simulations is at the back of the bulbous head; e.g., around $x=1000$ and $\mathrm{t}=500$. The discrete particle simulations display smoother behaviour than the continuum model, where the mass of the particles is also slightly more in the back compared to the numerical solution of the continuum model. We suspect the disagreement in the continuum and discrete particle simulations stems from the fact that the segregationstructure in the front is approximated by a shock rather than the full structure of the breaking-size-segregation wave, that is expected at the back of the bulbous head [8890]. This difference modified the effective basal friction; and, hence, the bulk dynamics and shape of the front. To remove the discontinuity in the first derivative of the heightprofile, at the top of the bulbous head, one would need a more complex model that retains more details of the vertical segregation structure. A more detailed discussion of the segregating-structure can be found in section 3.5.2.

Figure 3.7 shows a second comparison with particle simulations for the case $h^{\text {in }}=$ 7.4, $\alpha_{s}=0.08$. These parameters result in sub-critical inflow conditions; however the structure is still very similar with a bulbous head forming and growing longer over time. In this case, the height is slightly under-predicted, in contrast to the super-critical case, see figure 3.6. The height-profile seems to be less steep in the continuum model compared to the discrete particle simulations. Furthermore, the continuum model overpredicts the flow velocity, which can partially be explained by the fact that the flow is subcritical. In the particle simulations the maser boundary is constructed such that it can interact with the main flow particles. The flow-front is more resistive, due to evolving segregation profile, which feedbacks on the maser boundary lowering the inflow velocity produced by the maser. Therefore, we see that the inflow velocity is decreasing with time, in the discrete particle simulations, and lower than the steady-flow relation, (3.28), used by the continuum model. This is similar to the complex feedback between segregation profile, friction and hence velocity, seen in the breaking size-segregation structures studied by [88] and is not captured by the current continuum models.

Further study is needed to investigate the limits of the current model with respect to the inflow height, Froude number and chute angle. Moreover, the inflow conditions for sub-critical flow-configurations should be examined. 

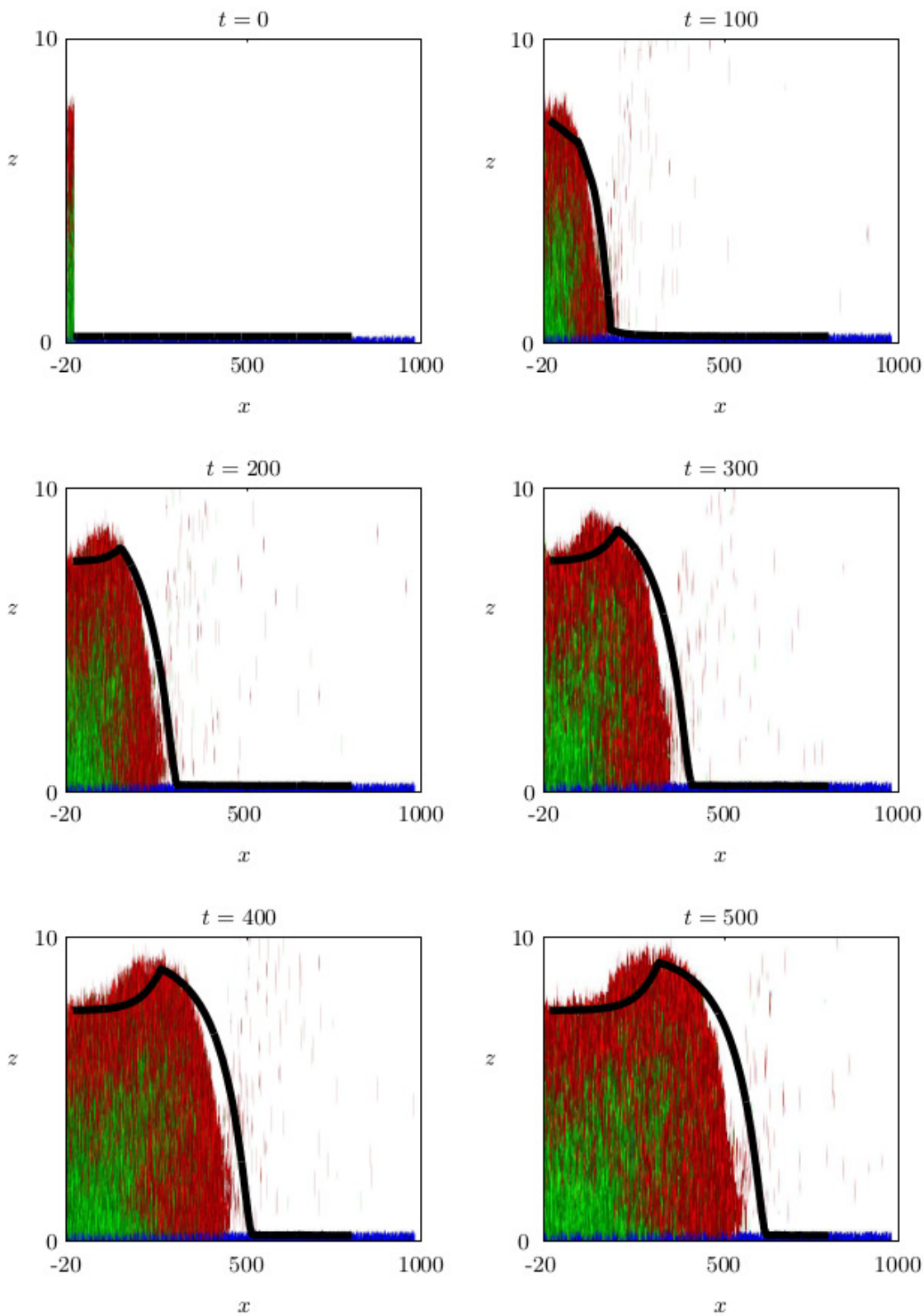

Figure 3.7: Snapshots of discrete particle simulation of a bidisperse chute flow over a rough bottom at various times The inflow height is 7.4 particles diameters, resulting in subcritical inflow and the mixture is $50 / 50$ large and small particles by volume. Red are the large particles, green are the small particles. The black lines denote the DGFEM solution for the height. Both $x$ and $z$ are scaled by the large particle diameter $d^{* L}$, in both the discrete particle simulations and DGFEM solutions. Note, that the $x$-axis is squeezed by a factor 100 compared to the $z$-axis, so the individual particles appear to be very thin in this plot. 


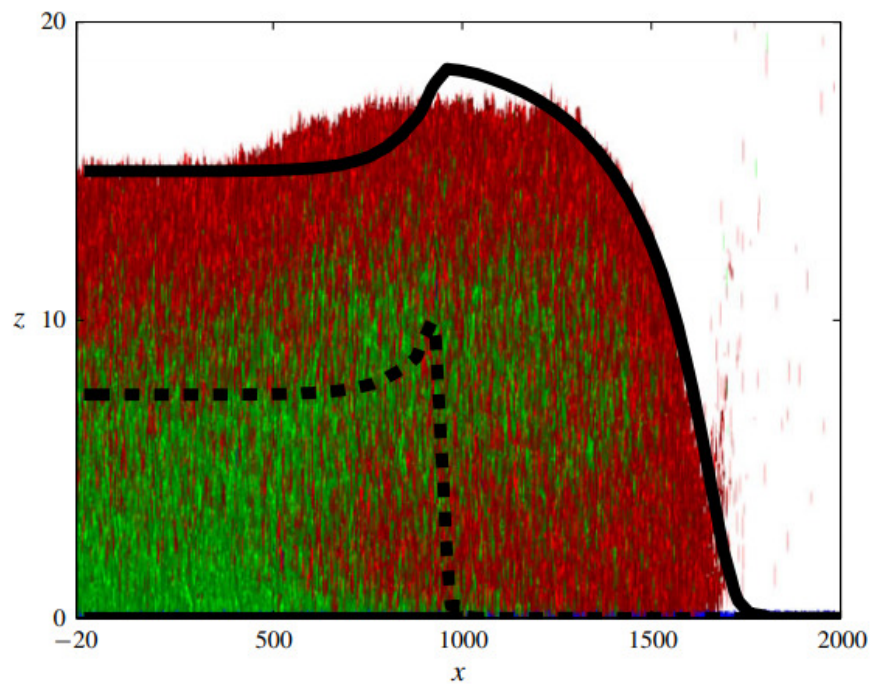

Figure 3.8: Enlarged image of the $t=500$ snapshot from figure 3.6. Red are the large particles, green are the small particles. The black lines denote the DGFEM solution for the height (solid) and height of small particles (dashed). Note the breaking size-segregation wave in the discrete particle simulations between $x \approx 500$ and $x \approx 1500$.

\subsubsection{SEgREgation PROFILES}

Looking at the segregation profiles from the discrete particle simulations in more detail, we see that the shock in the small particle concentration is unphysical and that the region with only large particles is much smaller than predicted by the continuum model. In general, the segregation profiles in the discrete particle simulations are not as sharp due to diffusive remixing, and possibly some deposition of large particles at the front with re-circulation. In the front of the flow, we see a thin band consisting of only large particles. Behind that, there is an expanding structure around the shock position, see figure 3.8. The structure observed here in the discrete particle simulations has been predicted from a non-depth-averaged continuum model $[41,89,90]$ and in both experiments and discrete particle simulations of a moving belt [88]. This structure was named the breaking size-segregation wave and its study is ongoing. Nevertheless, these results indicate that the assumption of full segregation needs to be relaxed in order to obtain a better continuum model for the segregation behaviour in this region.

\subsection{CONCLUSION AND DISCUSSION}

Granular flows containing a particle-size distribution, in both laboratory experiments and the natural environment, often show a bulbous head structure at their flow front. Using three-dimensional discrete particle simulations, and a simple one-dimensional depth-averaged continuum model, we have shown that this bulbous head structure is predicted at both the discrete and the continuum level. Furthermore, our long-time numerical solutions of the continuum model converge to a novel combination of two 
travelling wave solutions. This allows for an efficient computation of key features of the flow, such as the maximum flow depth and the mass flux in steady-state.

The simple one-dimensional depth-averaged model, that we present for this complex problem, is calibrated using only small-scale, steady-state, discrete particle simulations, i.e. there are no fitting parameters. We performed a preliminary comparison (one super- and one sub-critical) between the computationally cheap depth-averaged model (requiring minutes of computation on a single core) with two large expensive (requiring several months of computation on a single core) fully three dimensional particle simulations. For the super-critical flow case the depth-averaged model was able to capture key flow properties e.g. maximum flow depth, and propagating speed; however, it was not able to accurately capture the details of the segregating profile. For the sub-critical case discrepancies arose and increased with time; the problem, lying with the inflow boundary condition for the depth-averaged continuum model. A more detailed comparison between the continuum model and the full scale particle simulations should be undertaken and the full range of validity of the simple continuum model determined. However, given the efficiency of the model it could be an elegant tool for investigating the leading-order behaviour of complex segregating geophysical flows in the future.

As stated above, despite capturing various bulk flow properties the current continuum model does not capture all the details of the segregation behaviour, since it assumes full segregation at every position. There are many ways the model could be improved with respect to the segregation-profile. Firstly, one could insert a diffusion layer between the pure-large and pure-small phases as done by Edwards and Vriend [58]. Alternatively, one could fit the 'correct' two-dimensional breaking size-segregation wave profile $[41,88-90]$ at the one-dimensional shock position. This structure is known to appear in similar systems, both in discrete particle simulations and solutions of the full two-dimensional segregation equation. Furthermore, the breaking size-segregation wave becomes a simple shock when the segregation equation is depth-averaged $[57,91]$. Finally, we could even couple the two-dimensional segregation equation to the onedimensional depth-averaged bulk flow model. For example, one could construct a two dimensional domain which is bounded by the flow-base, inflow-boundary and the onedimensional height profile. On this domain the two-dimensional segregation model can then be solved numerically, using a velocity profile reconstructed from the computed depth-averaged velocity via (3.4). This formulation would allow the diffusion terms to be retained within the segregation model and hence diffusive remixing effects could also be captured.

From a mathematical point of view, it would be interesting to check under which conditions our depth-averaged model is well-posed. The closely related model of Woodhouse et al. [59] is ill-posed when the characteristic from the segregation equation coincides with one of the characteristics of the bulk flow model, i.e. when the model fails to be strictly hyperbolic. However, it becomes unconditionally well-posed if a viscosity term is added to the momentum balance [6]. Thus, another possible step is to see what happens to the travelling wave solution if such a viscous term is added to our model. The addition of this term has recently been investigated for mono-disperse flows by Gray and Edwards [92] and by Baker et al. [6] for the model of Woodhouse et al. [59].

Special attention should also be paid to the use of the shape factor, $\alpha \neq 1$, as this 
makes the complexity of the computational algorithm for the continuum method a lot higher; requiring the use of a more sophisticated wetting and drying treatment (see appendix 3.A.4). The inclusion of the non-unity shape-factor creates a thin precursor layer which causes a strong restriction on the time-step of the numerical DGFEM solutions. The numerical solution of the continuum model is closer to the particle simulations with the inclusion of the 'correct' shape factor; but, the general structure of the height and velocity of the bulbous head are all captured by the approximate case $\alpha=1$. A more detailed comparison between the DGFEM and particle simulations forms the central theme of future work where the cost versus error of including a non-unity shape factor will be further investigated.

For obtaining accurate closure relations and performing a detailed comparison between discrete and continuum models, it is helpful to coarse-grain the discrete particle simulations. Coarse-graining allows us to extract three-dimensional continuum fields, from the discrete particle data, for e.g. the momentum and stresses [93-95], both for the flow as a whole and for each constituent separately [96]. This, in turn, can show us which assumptions in the continuum model are most restrictive and help us to improve full three-dimensional as well as shallow granular segregation models. Currently, coarse-grained fields have not been constructed for the system studied here.

To make the depth-averaged shallow granular flow framework more general, it can be extended from bidisperse to tri- and polydisperse mixtures, for which segregation frameworks have been developed by Gray and Ancey [97] and Marks et al. [51], respectively. Both models have the same structure as the current model for bidisperse flows, and it would therefore be relatively straightforward to perform the same analysis as is done in this paper. It has also been shown by Schlick et al. [98] that such models can predict segregating patterns in the formation of heaps, when first calibrated with discrete particle simulations. Due to of the weak coupling between the bulk flow model and the segregation model, one could even think of coupling a depth-averaged segregation model, such as the one of [57], to a model for water-saturated granular chute flows, for example the model described in Kowalski and McElwaine [99].

Currently, the calibration of the constitutive relations for our chute flow model has to be repeated for each different combination of materials and particle sizes. To make the continuum model more applicable, the friction laws for bidisperse flows should be validated and extended to varying size ratios and mixture compositions. This can be done by determining a more general friction law, or by designing efficient discrete particle simulations that can determine the friction coefficient on the fly. The same holds for the a-priori unknown velocity profile, which generally depends on many parameters, such as base geometry and height. Baker et al. [6] already developed a framework for the depth-averaged segregation equation with a general velocity profile, and extending this work is an interesting avenue of future research.

\section{ACKNowledgements}

The authors would like to thank Chris G. Johnson for interesting conversations about this work and the Dutch Technology Foundation TTW (formally STW) for its financial support via the STW-Vidi project 13472, Shaping Segregation: Advanced Modelling of Segregation and its Application to Industrial Processes. Nico Gray was supported by NERC 
grants NE/E003206/1 and NE/K003011/1, EPSRC grant EP/M022447/1 as well as a Royal Society Wolfson Research Merit Award WM150058.

\section{REFERENCES}

[1] I. F. C. Denissen, T. Weinhart, A. Te Voortwis, S. Luding, J. M. N. T. Gray, and A. R. Thornton, Bulbous head formation in bidisperse shallow granular flow over an inclined plane, Journal of Fluid Mechanics 866, 263 (2019).

[2] K. Dalbey, A. K. Patra, E. B. Pitman, M. I. Bursik, and M. F. Sheridan, Input uncertainty propagation methods and hazard mapping of geophysical mass flows, Journal of Geophysical Research: Solid Earth 113 (2008).

[3] R. M. Iverson, M. Logan, R. G. LaHusen, and M. Berti, The perfect debris flow? aggregated results from 28 large-scale experiments, Journal of Geophysical Research: Earth Surface 115 (2010).

[4] O. Pouliquen and J. W. Vallance, Segregation induced instabilities of granular fronts, Chaos 9, 621 (1999).

[5] J. W. Vallance and S. B. Savage, Particle segregation in granular flows down chutes, in IUTAM Symposium on Segregation in Granular Flows (Springer, 2000) pp. 31-51.

[6] J. L. Baker, C. G. Johnson, and J. M. N. T. Gray, Segregation-induced finger formation in granular free-surface flows, Journal of Fluid Mechanics 809, 168 (2016).

[7] G. V. Middleton, Experimental Studies Related to Problems of Flysch Sedimentation,, Vol. 7 (The Geological Association of Canada, 1970) pp. 253-272.

[8] J. M. N. T. . Gray and V. A. Chugunov, Particle-size segregation and diffusive remixing in shallow granular avalanches, Journal of Fluid Mechanics 569, 365 (2006).

[9] S. B. Savage and C. K. K. Lun, Particle size segregation in inclined chute flow of dry cohesionless granular solids, Journal of Fluid Mechanics 189, 311 (1988).

[10] S. Dippel and S. Luding, Simulations on size segregation: Geometrical effects in the absence of convection, J. Physics. I France 5, 1527 (1995).

[11] S. Luding, E. Clément, J. Rajchenbach, and J. Duran, Simulations of pattern formation in vibrated granular media, EPL (Europhysics Letters) 36, 247 (1996).

[12] J. T. Jenkins, Particle segregation in collisional flows of inelastic spheres, in Physics of dry granular media, NATO ASI series, Vol. 350, edited by Herrmann, Hovi, and Luding (Kluwer, 1998) pp. 645-658.

[13] T. Mullin, Coarsening of self-organised clusters in binary particle mixtures, Phys. Rev. Lett. 84, 4741 (2000).

[14] M. E. Mobius, B. E. Lauderdale, S. R. Nageland, and H. M. Jaeger, Size separation of granular particles, Nature , 270 (2001). 
[15] D. C. Hong, P. V. Quinn, and L. S., Reverse brazil nut problem: Competition between percolation and condensation, Phys. Rev. Lets. 86, 3423 (2001).

[16] J. T. Jenkins and D. K. Yoon, Segregation in binary mixtures under gravity, Phys. Rev. Lett. 88, 1 (2002).

[17] S. González, C. R. K. Windows-Yule, S. Luding, D. J. Parker, and A. R. Thornton, Forced axial segregation in axially inhomogeneous rotating systems, Phys. Rev. E 92 , 022202 (2015).

[18] C. R. K. Windows-Yule, B. J. Scheper, A. J. van der Horn, N. Hainsworth, J. Saunders, D. J. Parker, and A. R. Thornton, Understanding and exploiting competing segregation mechanisms in horizontally rotated granular media, New Journal of Physics 18, 023013 (2016).

[19] D. V. Khakhar, J. J. McCarthy, and J. M. Ottino, Mixing and segregation of granular materials in chute flows, Chaos: An Interdisciplinary Journal of Nonlinear Science 9, 594 (1999).

[20] Y. Fan and K. M. Hill, Theory for shear-induced segregation of dense granular mixtures, New journal of physics 13, 095009 (2011).

[21] L. Staron and J. C. Phillips, Stress partition and microstructure in size-segregating granular flows, Phys. Rev. E 92, 022210 (2015).

[22] M. Larcher and J. T. Jenkins, Segregation and mixture profiles in dense, inclined flows of two types of spheres, Physics of Fluids 25, 113301 (2013).

[23] R. M. Iverson, The debris-flow rheology myth, in Debris flow Mechanics and Mitigation Conference, Mills Press, Davos (2003) pp. 303-314.

[24] K. A. Gillemot, E. Somfai, and T. Börzsönyi, Shear-driven segregation of dry granular materials with different friction coefficients, Soft matter 13, 415 (2017).

[25] K. van der Vaart, M. P. van Schrojenstein Lantman, T. Weinhart, S. Luding, C. Ancey, and A. R. Thornton, Segregation of large particles in dense granular flows suggests a granular saffman effect, Physical Review Fluids 3, 074303 (2018).

[26] T. Weinhart, A. R. Thornton, S. Luding, and O. Bokhove, Closure relations for shallow granular flows from particle simulations, Granular Matter 14, 531 (2012).

[27] A. R. Thornton, T. Weinhart, S. Luding, and O. Bokhove, Frictional dependence of shallow-granular flows from discrete particle simulations, The European Physical Journal E 35, 1 (2012).

[28] L. Staron and J. C. Phillips, How large grains increase bulk friction in bi-disperse granular chute flows, Computational Particle Mechanics , 1 (2015).

[29] R. M. Iverson, The physics of debris flows, Reviews of geophysics 35, 245 (1997). 
[30] B. P. Kokelaar, R. L. Graham, J. M. N. T. Gray, and J. W. Vallance, Fine-grained linings of leveed channels facilitate runout of granular flows, Earth and Planetary Science Letters 385, 172 (2014).

[31] T. Takahashi, Debris Flow: Mechanics, Prediction and Countermeasures, 2nd edition (CRC Press, 2014).

[32] C. G. Johnson, B. P. Kokelaar, R. M. Iverson, M. Logan, R. G. LaHusen, and J. M. N. T. Gray, Grain-size segregation and levee formation in geophysical mass flows, Journal of Geophysical Research: Earth Surface 117 (2012).

[33] T. Haas, L. Braat, J. R. F. W. Leuven, I. R. Lokhorst, and M. G. Kleinhans, Effects of debris flow composition on runout, depositional mechanisms, and deposit morphology in laboratory experiments, Journal of Geophysical Research: Earth Surface 120, 1949 (2015).

[34] M. Logan and R. Iverson, Video documentation of experiments at the usgs debrisflow flume 2008, U.S. Geological Survey Open-File Report 2007-1315 v. 1.3. (2013).

[35] T. R. H. Davies, Debris-flow surges - experimental simulation, Journal of Hydrology (New Zealand) , 18 (1990).

[36] R. M. Iverson and R. G. LaHusen, Friction in debris flows: Inferences from large-scale flume experiments, American Society of Civil Engineers (Ed.), Hydraulic Engineering 93 (1993).

[37] D. Berzi and J. T. Jenkins, Steady inclined flows of granular-fluid mixtures, Journal of Fluid Mechanics 641, 359 (2009).

[38] R. Delannay, A. Valance, A. Mangeney, O. Roche, and P. Richard, Granular and particle-laden flows: from laboratory experiments to field observations, Journal of Physics D: Applied Physics 50, 053001 (2017).

[39] S. A. Dunning and P. J. Armitage, The grain-size distribution of rock-avalanche deposits: implications for natural dam stability, in Natural and artificial rockslide dams (Springer, 2011) pp. 479-498.

[40] C. Goujon, B. Dalloz-Dubrujeaud, and N. Thomas, Bidisperse granular avalanches on inclined planes: A rich variety of behaviors, The European Physical Journal E 23, 199 (2007).

[41] J. M. N. T. Gray and C. Ancey, Segregation, recirculation and deposition of coarse particles near two-dimensional avalanche fronts, Journal of Fluid Mechanics 629, 387 (2009).

[42] C. S. Campbell, P. W. Cleary, and M. Hopkins, Large-scale landslide simulations: Global deformation, velocities and basal friction, Journal of Geophysical Research: Solid Earth 100, 8267 (1995). 
[43] S. Luding, Cohesive, frictional powders: contact models for tension, Granular matter 10, 235 (2008).

[44] A. R. Thornton, T. Weinhart, S. Luding, and O. Bokhove, Modeling of particle size segregation: calibration using the discrete particle method, International Journal of Modern Physics C 23, 1240014 (2012).

[45] S. B. Savage and K. Hutter, The motion of a finite mass of granular material down a rough incline, Journal of fluid mechanics 199, 177 (1989).

[46] J. M. N. T. Gray, Y.-C. Tai, and S. Noelle, Shock waves, dead zones and particle-free regions in rapid granular free-surface flows, Journal of Fluid Mechanics 491, 161 (2003).

[47] O. Bokhove and A. R. Thornton, Shallow granular flows, in Handbook of Environmental Fluid Dynamics, edited by H. Fernando (CRC Press, 2012).

[48] J. Bridgwater, W. S. Foo, and D. J. Stephens, Particle mixing and segregation in failure zones—theory and experiment, Powder technology 41, 147 (1985).

[49] J. M. N. T. Gray and A. R. Thornton, A theory for particle size segregation in shallow granular free-surface flows, Proceedings of the Royal Society of London A: Mathematical, Physical and Engineering Sciences 461, 1447 (2005).

[50] A. R. Thornton, J. M. N. T. Gray, and A. J. Hogg, A three-phase mixture theory for particle size segregation in shallow granular free-surface flows, Journal of Fluid Mechanics 550, 1 (2006).

[51] B. Marks, P. Rognon, and I. Einav, Grainsize dynamics of polydisperse granular segregation down inclined planes, Journal of Fluid Mechanics 690, 499 (2012).

[52] D. Tunuguntla, O. Bokhove, and A. Thornton, A mixture theory for size and density segregation in shallow granular free-surface flows, Journal of fluid mechanics $\mathbf{7 4 9}$, 99 (2014).

[53] D. R. Tunuguntla, T. Weinhart, and A. R. Thornton, Comparing and contrasting sizebased particle segregation models, Computational Particle Mechanics , 1 (2016).

[54] K. M. Hill and Y. Fan, Granular temperature and segregation in dense sheared particulate mixtures, KONA Powder and Particle Journal 33, 150 (2016).

[55] M. Larcher and J. T. Jenkins, The evolution of segregation in dense inclined flows of binary mixtures of spheres, Journal of Fluid Mechanics 782, 405 (2015).

[56] T. Takahashi, H. Nakagawa, T. Harada, and Y. Yamashiki, Routing debris flows with particle segregation, Journal of Hydraulic Engineering 118, 1490 (1992).

[57] J. M. N. T. Gray and B. P. Kokelaar, Large particle segregation, transport and accumulation in granular free-surface flows, Journal of Fluid Mechanics 652, 105 (2010). 
[58] A. N. Edwards and N. M. Vriend, Size segregation in a granular bore, Physical Review Fluids 1, 064201 (2016).

[59] M. J. Woodhouse, A. R. Thornton, C. G. Johnson, B. P. Kokelaar, and J. M. N. T. Gray, Segregation-induced fingering instabilities in granular free-surface flows, Journal of Fluid Mechanics 709, 543 (2012).

[60] S. Wiederseiner, N. Andreini, G. Épely-Chauvin, G. Moser, M. Monnereau, J. M. N. T. Gray, and C. Ancey, Experimental investigation into segregating granular flows down chutes, Physics of Fluids 23, 013301 (2011).

[61] P. G. Rognon, F. Chevoir, H. Bellot, F. Ousset, M. Naaïm, and P. Coussot, Rheology of dense snow flows: Inferences from steady state chute-flow experiments, Journal of Rheology 52, 729 (2008).

[62] A. Tripathi and D. V. Khakhar, Rheology of binary granular mixtures in the dense flow regime, Physics of Fluids 23, 113302 (2011).

[63] L. E. Silbert, J. W. Landry, and G. S. Grest, Granular flow down a rough inclined plane: transition between thin and thick piles, Physics of Fluids 15, 1 (2003).

[64] GDR-MiDi, On dense granular flows, The European Physical Journal E 14, 341 (2004).

[65] L. E. Silbert, D. Ertaş, G. S. Grest, T. C. Halsey, D. Levine, and S. J. Plimpton, Granular flow down an inclined plane: Bagnold scaling and rheology, Physical Review E 64, 051302 (2001).

[66] N. Brodu, P. Richard, and R. Delannay, Shallow granular flows down flat frictional channels: Steady flows and longitudinal vortices, Physical Review E 87, 022202 (2013).

[67] T. Faug, P. Childs, E. Wyburn, and I. Einav, Standing jumps in shallow granular flows down smooth inclines, Physics of Fluids 27, 073304 (2015).

[68] Y. Forterre and O. Pouliquen, Long-surface-wave instability in dense granular flows, Journal of Fluid Mechanics 486, 21 (2003).

[69] G. Saingier, S. Deboeuf, and P.-Y. Lagrée, On the front shape of an inertial granular flow down a rough incline, Physics of Fluids 28, 053302 (2016).

[70] O. Pouliquen, Scaling laws in granular flows down rough inclined planes, Physics of Fluids 11, 542 (1999).

[71] P. Jop, Y. Forterre, and O. Pouliquen, Crucial role of sidewalls in granular surface flows: consequences for the rheology, Journal of Fluid Mechanics 541, 167 (2005).

[72] B. Kokelaar, R. Bahia, K. Joy, S. Viroulet, and J. Gray, Granular avalanches on the moon: Mass-wasting conditions, processes and features, Journal of Geophysical Research: Planets (2017). 
[73] A. te Voortwis, Closure laws for granular, shallow-layer, bi-disperse flows down an inclined chute, Master's thesis, MSM Group, Univ. Twente, the Netherlands (2013).

[74] T. Weinhart, A. R. Thornton, S. Luding, and O. Bokhove, From discrete particles to continuum fields near a boundary, Granular Matter 14, 289 (2012).

[75] T. Weinhart, D. R. Tunuguntla, M. P. van Schrojenstein-Lantman, A. J. van der Horn, I. F. C. Denissen, C. R. Windows-Yule, A. C. de Jong, and A. R. Thornton, MercuryDPM: A Fast and Flexible Particle Solver Part A: Technical Advances, in Proceedings of the 7th International Conference on Discrete Element Methods (Springer Singapore, Singapore, 2017) pp. 1353-1360.

[76] MercuryDPM, https : //www . mercurdpm . org, last accessed on August 27th 2019.

[77] W. H. Reed and T. R. Hill, Triangular mesh methods for the neutron transport equation, Tech. Rep. (Los Alamos Scientific Laboratory, 1973).

[78] C. W. Shu, A brief survey on discontinuous Galerkin methods in computational fluid dynamics, Advances in Mechanics, v43 , 541 (2013).

[79] A. Harten, P. D. Lax, and B. v. Leer, On upstream differencing and Godunov-type schemes for hyperbolic conservation laws, SIAM review 25, 35 (1983).

[80] B. Cockburn and C. W. Shu, TVB Runge-Kutta local projection discontinuous Galerkin finite element method for conservation laws. II. General framework, Mathematics of computation 52, 411 (1989).

[81] S. Bunya, E. J. Kubatko, J. J. Westerink, and C. Dawson, A wetting and drying treatment for the Runge-Kutta discontinuous Galerkin solution to the shallow water equations, Computer Methods in Applied Mechanics and Engineering 198, 1548 (2009).

[82] L. Pesch, A. Bell, W. Sollie, V. Ambati, O. Bokhove, and J. van der Vegt, $h p G E M-A$ software framework for discontinuous Galerkin finite element methods, ACM Transactions on Mathematical Software 33(4) (2007).

[83] S. Nurijanyan, Discrete and continuous Hamiltonian systems for wave modelling, Ph.D. thesis, University of Twente, Enschede (2013).

[84] M. O. Bristeau and B. Coussin, Boundary conditions for the shallow water equations solved by kinetic schemes, Ph.D. thesis, INRIA (2001).

[85] A. J. Hogg and D. Pritchard, The effects of hydraulic resistance on dam-break and other shallow inertial flows, Journal of Fluid Mechanics 501, 179 (2004).

[86] O. Pouliquen, On the shape of granular fronts down rough inclined planes, Physics of Fluids 11, 1956 (1999).

[87] R. J. LeVeque, Finite volume methods for hyperbolic problems, Vol. 31 (Cambridge university press, 2002). 
[88] K. van der Vaart, A. Thornton, C. Johnson, T. Weinhart, L. Jing, P. Gajjar, J. Gray, and C. Ancey, Breaking size-segregation waves and mobility feedback in dense granular avalanches, Granular matter 20, 46 (2018).

[89] A. R. Thornton and J. M. N. T. Gray, Breaking size segregation waves and particle recirculation in granular avalanches, Journal of Fluid Mechanics 596, 261 (2008).

[90] P. Gajjar, K. van der Vaart, A. R. Thornton, C. G. Johnson, C. Ancey, and J. M. N. T. Gray, Asymmetric breaking size-segregation waves in dense granular free-surface flows, Journal of Fluid Mechanics 794, 460 (2016).

[91] J. M. N. T. Gray and B. P. Kokelaar, Large particle segregation, transport and accumulation in granular free-surface flows-erratum, Journal of Fluid Mechanics 657, 539 (2010).

[92] J. M. N. T. Gray and A. N. Edwards, A depth-averaged-rheology for shallow granular free-surface flows, Journal of Fluid Mechanics 755, 503 (2014).

[93] M. Babic, Average balance equations for granular materials, International journal of engineering science 35, 523 (1997).

[94] I. Goldhirsch, Stress, stress asymmetry and couple stress: from discrete particles to continuous fields, Granular Matter 12, 239 (2010).

[95] T. Weinhart, R. Hartkamp, A. R. Thornton, and S. Luding, Coarse-grained local and objective continuum description of three-dimensional granular flows down an inclined surface, Physics of Fluids 25, 070605 (2013).

[96] D. R. Tunuguntla, A. R. Thornton, and T. Weinhart, From discrete elements to continuum fields: Extension to bidisperse systems, Computational particle mechanics 3, 349 (2016).

[97] J. M. N. T. Gray and C. Ancey, Multi-component particle-size segregation in shallow granular avalanches, Journal of Fluid Mechanics 678, 535 (2011).

[98] C. P. Schlick, A. B. Isner, B. J. Freireich, Y. Fan, P. B. Umbanhowar, J. M. Ottino, and R. M. Lueptow, A continuum approach for predicting segregation in flowing polydisperse granular materials, Journal of Fluid Mechanics 797, 95 (2016).

[99] J. Kowalski and J. N. McElwaine, Shallow two-component gravity-driven flows with vertical variation, Journal of Fluid Mechanics 714, 434 (2013). 


\section{APPENDiX}

\section{A. DGFEM DISCRETISATION}

In this appendix, we will give a detailed overview of the discontinuous Galerkin finite element method that is used for the numerical solutions in section 3.3. First of all, note that equations (3.6)-(3.9) can be written in the standard hyperbolic form

$$
\frac{\partial U}{\partial t}+\frac{\partial}{\partial x} f(U)=s(U)
$$

where

$$
U=\left(\begin{array}{c}
h \\
h \bar{u} \\
h \bar{\phi}
\end{array}\right), \quad f(U) \quad=\left(\begin{array}{c}
\alpha h \bar{u}^{2}+\frac{1}{2} \cos \theta h^{2} \\
h \bar{\phi} \bar{u}-\left(1-\alpha_{s}\right) h \bar{\phi} \bar{u}(1-\bar{\phi})
\end{array}\right), \quad s(U)=\left(\begin{array}{c}
0 \\
h S(h, \bar{u}, \bar{\phi}) \\
0
\end{array}\right) .
$$

Here, $h>0, \alpha \geq 1,0^{\circ} \leq \theta<90^{\circ}$ and $0 \leq \alpha_{s} \leq 1$. The eigenvalues of this system are, in no particular order,

$$
\begin{aligned}
& \lambda_{1}=\alpha \bar{u}-\sqrt{\left(\alpha^{2}-\alpha\right) \bar{u}^{2}+h \cos \theta}, \\
& \lambda_{2}=\alpha \bar{u}+\sqrt{\left(\alpha^{2}-\alpha\right) \bar{u}^{2}+h \cos \theta}, \\
& \lambda_{3}=\bar{u}\left(\alpha_{s}+2 \bar{\phi}-2 \alpha_{s} \bar{\phi}\right) .
\end{aligned}
$$

Since all eigenvalues are real, this system of equations is indeed hyperbolic.

\section{A.1. NotaTion}

A given one-dimensional domain $\Omega \subset \mathbb{R}$ is divided into equal-sized, non-overlapping intervals $K$. The set of all of these elements is denoted as $\mathscr{E}$. Each element has a boundary $\partial K$, which consists of the two endpoints of the interval. Each point of the boundary of an element has an outward unit normal vector $\mathbf{n}$. We approximate $U$ by its discrete counterpart $U_{h} \in\left(V_{h}\right)^{3}$, in which $V_{h}$ is the space of piecewise linear polynomials. The functions in $V_{h}$ may be discontinuous across faces, which distinguishes discontinuous Galerkin finite element methods from conforming finite element methods. Note, that in this case, the value of $U_{h}(x)$ on an element $K$ can be expressed as $\mathbf{U}_{K}^{0} \phi^{0}(x)+\mathbf{U}_{K}^{1} \phi^{1}(x)$ with the basis functions $\phi^{0}$ and $\phi^{1}$ linearly independent linear polynomials and coefficients $\mathbf{U}_{K}^{\ell} \in \mathbb{R}^{3}, \ell=0,1$.

\section{A.2. DisCRETISATION}

We now put the system into a discrete weak form. Multiply each equation in (3.40) with an arbitrary test function $v \in V_{h}$ and integrate over each element, which results in

$$
\int_{K} \frac{\partial}{\partial t}\left(U_{h}\right) v+\frac{\partial}{\partial x} F\left(U_{h}\right) v d x=\int_{K} S\left(U_{h}\right) v d x, \quad K \in \mathscr{E} .
$$


Integrating the second term by parts yields:

$$
\int_{K} \frac{\partial}{\partial t}\left(U_{h}\right) v-F\left(U_{h}\right) \frac{\partial}{\partial x} v d x+\int_{\partial K} F\left(U_{h}\right) v \mathbf{n} d s=\int_{K} S\left(U_{h}\right) v d x, \quad K \in \mathscr{E} .
$$

There are discontinuities in $U_{h}$ allowed on the element faces $\partial K$, therefore the flux $F\left(U_{h}\right)$ is replaced by a numerical flux $\hat{F}\left(U_{h}^{L}, U_{h}^{R}\right)$, where $U_{h}^{L}$ and $U_{h}^{R}$ are the values at $\partial K$ of the left and right element respectively, giving the discretisation

$$
\int_{K} \frac{\partial}{\partial t}\left(U_{h}\right) v-\int_{K} F\left(U_{h}\right) \frac{\partial}{\partial x} v d x+\int_{\partial K} \hat{F}\left(U_{h}^{L}, U_{h}^{R}\right) v \mathbf{n} d s=\int_{K} S\left(U_{h}\right) v d x, \quad K \in \mathscr{E} .
$$

Here we use the numerical flux of Harten et al. [1], which is positivity preserving and works well for the similarly structured 1D shallow water equations $[2,3]$. For completeness, this is repeated here. Define $a_{L}=\alpha \bar{u}-\sqrt{\left(\alpha^{2}-\alpha\right) \bar{u}^{2}+h \cos \theta}=\lambda_{1}$ and $a_{R}=\alpha \bar{u}+$ $\sqrt{\left(\alpha^{2}-\alpha\right) \bar{u}^{2}+h \cos \theta}=\lambda_{2}$ as the characteristic wave speeds of the bulk flow and define $f_{L}=F\left(U_{h}^{L}\right)$ and $f_{R}=f\left(U_{h}^{R}\right)$, respectively, the HLL flux is then given by

$$
\hat{F}\left(U_{h}^{L}, U_{h}^{R}\right)= \begin{cases}f_{L} & \text { if } 0 \leq a_{L}<a_{R}, \\ \frac{-a_{L}}{a_{R}-a_{L}} f_{R}+\frac{a_{R}}{a_{R}-a_{L}} f_{L}+\frac{a_{L} a_{R}}{a_{R}-a_{L}}\left(U_{h}^{R}-U_{h}^{L}\right) & \text { if } a_{L}<0<a_{R}, \\ f_{R} & \text { if } a_{L}<a_{R} \leq 0 .\end{cases}
$$

\section{A.3. SLOPE LIMITING}

Since the system is hyperbolic, shocks in the solution can form over time. This can lead to severe oscillations near the shock, which can be prevented by using a slope limiter. Here, the TVB limiter of Cockburn and Shu [4] is used.

\section{A.4. WETTING AND DRYING TREATMENT}

Physically, the height of the particle flow can never become negative, so this should also be enforced in the numerical solutions of the continuum model. In order to do so, we use the wetting and drying treatment of Bunya et al. [5], which conserves mass and, if the average height in an element is large enough, momentum. It is a shallow layer approach, in the sense that if the height at a node is below a certain threshold, the slope of the height and momentum are changed such that the height is above that threshold everywhere. If the average height in an element is below that threshold, the height in that element is set to its average and the momentum is set to 0 . Note, that we expect a very thin precursor layer in front of the flow, since $\alpha \neq 1$ [6]. This layer can be much smaller than the numerical precision, so it is important to correct the height every time step. As the gradient of the height also goes to zero, we must not be too aggressive with our limiting, as this causes numerical artifacts where the gradient of the height gets the wrong sign for some elements. Here, the threshold is chosen to be $10^{-10}$, which is well above numerical precision, but low enough that numerical artifacts are minimal.

\section{A.5. TIME INTEGRATION}

With some basic manipulation, the system of equations (3.43) can be written as

$$
\frac{\partial \mathbf{U}}{\partial t}=\mathscr{L}(\mathbf{U})
$$


where $\mathbf{U}$ are the coefficients and $\mathscr{L}$ is some non-linear operator. We use the forward Euler method [7] to compute all coefficients at the next time step:

$$
\mathbf{U}^{n+1}=\Upsilon\left(\mathbf{U}^{n}+\Delta t \mathscr{L}\left(\mathbf{U}^{n}\right)\right),
$$

where $\Upsilon$ is the operator that denotes the combination of slope- and non-negativity limiting and $\Delta t$ is the time step. From experience we know that the most severe restriction on the time step comes from the wetting and drying treatment, so a higher order timeintegration method is not needed for a better stability and accuracy of the method.

\section{B. DETAils OF THE TRAVELLING WAVE SOLUTION}

\section{B.1. DERIVATION OF $\bar{u}^{\text {in }}$}

In this appendix, the exact formula for $\bar{u}^{\text {in }}$ such that $S\left(h^{\text {in }}, \bar{u}^{\text {in }}, \bar{\phi}^{\text {in }}\right)=0$, i.e. $\mu\left(h^{\text {in }}, \bar{u}^{\text {in }}, \bar{\phi}^{\text {in }}\right)=$ $\tan \theta$, will be derived. It is assumed that $h^{\text {in }}, \bar{\phi}^{\text {in }}$ and all friction and system parameters are known beforehand, so that $\bar{u}^{\text {in }}$ is the only unknown.

The friction law is given by

$$
\begin{aligned}
& \mu(h, \bar{u}, \bar{\phi})=\bar{\phi} \mu^{S}+(1-\bar{\phi}) \mu^{L}, \\
& \mu^{v}(h, \bar{u})=\tan \delta_{1}^{v}+\frac{\tan \delta_{2}^{v}-\tan \delta_{1}^{v}}{\frac{\beta^{v} h}{A^{v} d^{v} F}+1}, \quad v=S, L,
\end{aligned}
$$

so we have to solve

$$
\tan \theta=\bar{\phi}^{\text {in }}\left(\tan \delta_{1}^{S}+\frac{\tan \delta_{2}^{S}-\tan \delta_{1}^{S}}{\frac{\beta^{S} h^{\text {in }}}{A^{S} d^{S} F}+1}\right)+\left(1-\bar{\phi}^{\text {in }}\right)\left(\tan \delta_{1}^{L}+\frac{\tan \delta_{2}^{L}-\tan \delta_{1}^{L}}{\frac{\beta^{L} h^{\text {in }}}{A^{L} d^{L} F}+1}\right) .
$$

Introducing the following notation:

$$
\begin{aligned}
b & =\tan \theta-\bar{\phi}^{\mathrm{in}} \tan \delta_{1}^{S}-\left(1-\bar{\phi}^{\mathrm{in}}\right) \tan \delta_{1}^{L}, \\
a^{S} & =\bar{\phi}^{\mathrm{in}}\left(\tan \delta_{2}^{S}-\tan \delta_{1}^{S}\right), \\
a^{L} & =\left(1-\bar{\phi}^{\mathrm{in}}\right)\left(\tan \delta_{2}^{L}-\tan \delta_{1}^{L}\right), \\
c^{v} & =\frac{\beta^{v}}{A^{v} d^{v}}
\end{aligned}
$$$$
v=S, L \text {, }
$$

one can rewrite (3.47) as

$$
\frac{a^{S}}{c^{S} h^{\text {in }} / F+1}+\frac{a^{L}}{c^{L} h^{\text {in } / F+1}}=b .
$$

Rearrangement of the terms then shows that $F$ can be expressed as

$$
\begin{aligned}
\left(a^{L}+a^{S}-b\right) F & =\frac{h^{\text {in }}}{2}\left(\left(\left(b-a^{S}\right) c^{L}+\left(b-a^{L}\right) c^{S}\right)\right) \\
& +\frac{h^{\text {in }}}{2}\left(\sqrt{\left(\left(b-a^{S}\right) c^{L}+\left(b-a^{L}\right) c^{S}\right)^{2}-4\left(b-a^{L}-a^{S}\right) b c^{L} c^{S}}\right) .
\end{aligned}
$$

$\bar{u}^{\text {in }}$ can be determined via

$$
\bar{u}^{\text {in }}=F \sqrt{h^{\text {in }} \cos \theta} .
$$




\begin{tabular}{l|l|c|c} 
time & volume & difference to previous & $Q^{L} \cdot \Delta t$ \\
\hline 2000 & $4.42 \cdot 10^{4}$ & & \\
3000 & $6.62 \cdot 10^{4}$ & $2.20 \cdot 10^{4}$ & $2.20 \cdot 10^{4}$ \\
4000 & $8.82 \cdot 10^{4}$ & $2.20 \cdot 10^{4}$ & $2.20 \cdot 10^{4}$
\end{tabular}

Table 3.B.1: Volume difference in time-dependent DGFEM solutions

\section{B.2. CONSERVATION OF LARGE-PARTICLE VOLUME}

It must be noted that in the travelling wave solution of section 3.4, the values of $h$ and $\bar{u}$ for $\xi>0$ are constant, so the volume of the large particles in the front is infinite. However, there is a net flow of large particles towards the front, as can be seen in the time-dependent solutions of section 3.3. This transport rate for large particles $Q^{L}$ can be computed by taking the difference of the volumetric flow rate at the inflow $\xi=-\infty$ and outflow $\xi=\infty$ :

$$
\begin{aligned}
Q^{L}= & \int_{0}^{h^{\text {out }}}\left(u^{\text {out }}(z)-u_{f}\right)\left(1-\phi^{\text {out }}(z)\right) d z-\int_{0}^{h^{\text {in }}}\left(u^{\text {in }}(z)-u_{f}\right)\left(1-\phi^{\text {in }}(z)\right) d z, \\
= & h^{\text {out }} \bar{u}^{\text {out }}\left(\alpha_{s}\left(1-\bar{\phi}^{\text {out }}\right)+\left(1-\alpha_{s}\right)\left(1-\left(\bar{\phi}^{\text {out }}\right)^{2}\right)\right)-h^{\text {out }}\left(1-\bar{\phi}^{\text {out }}\right) u_{f} \\
& -h^{\text {in }} \bar{u}^{\text {in }}\left(\alpha_{s}\left(1-\bar{\phi}^{\text {in }}\right)+\left(1-\alpha_{s}\right)\left(1-\left(\bar{\phi}^{\text {in }}\right)^{2}\right)\right)-h^{\text {in }}\left(1-\bar{\phi}^{\text {in }}\right) u_{f},
\end{aligned}
$$

using the assumptions of a fully segregated flow and applying velocity profile (3.4). For the parameters in table 3.1, this gives the value $Q^{L}=22.0$. Since $Q^{L}>0$ in this situation, we expect the volume of the head to grow over time based on this analysis.

The front of the time-dependent DGFEM solutions are integrated numerically and evaluated at $t=2000,3000$ and 4000, see table 3.B.1. We see that the volume of the large particle front increases at a rate of $Q^{L} \cdot \Delta t$, which shows that the global mass balance and hence the travelling wave solution accurately predicts the growth of the head.

\section{C. Details OF Discrete PaRTicle Simulations}

In this appendix, details are given regarding the discrete particle simulations used to determine the friction parameters in table 3.1 and to produce figure 3.6. All simulations are performed with MercuryDPM [8-10], an open-source package designed for performing discrete particle simulations. It has been used for bidisperse chute-flows before, see e.g. [11].

\section{C.1. DEFINITIONS}

The system consists of two types of soft, spherical particles that have the same density, but different diameters and therefore different contact properties. On a microscopic scale, both types of particles have the same restitution coefficient and dimensionless contact time, but the large particles have a larger microscopic friction than the small particles, see section 3.C.2. Each particle $i$ has diameter $d_{i}$ and density $\rho^{p}$. The system variables are, for each particle $i$, its position $\mathbf{r}_{i}$, velocity $\mathbf{v}_{i}$ and angular velocity $\boldsymbol{\omega}_{i}$.

Each contact between two particles is treated as acting at a single point. The distance between two particles $i$ and $j$ is given by $r_{i j}=\left|\mathbf{r}_{i}-\mathbf{r}_{j}\right|$, and the overlap between these 
particles is $\delta_{i j}^{n}=\max \left(0,\left(d_{i}+d_{j}\right) / 2-r_{i j}\right)$. Note, that it is assumed that this overlap is small, otherwise contacts cannot be treated as being point-like. If two particles are in contact, one can define the unit normal vector $\hat{\mathbf{n}}_{i j}=\left(\mathbf{r}_{i}-\mathbf{r}_{j}\right) / r_{i j}$ and the vector from the centre of the particle to the contact point, the branch vector, $\mathbf{b}_{i j}=-\left(d_{i}-\delta_{i j}^{n}\right) \hat{\mathbf{n}}_{i j} / 2$. The relative velocity $\mathbf{v}_{i j}=\mathbf{v}_{i}-\mathbf{v}_{j}$ can be decomposed in a normal and tangential component as follows:

$$
\begin{aligned}
\mathbf{v}_{i j}^{n} & =\left(\mathbf{v}_{i j} \cdot \hat{\mathbf{n}}_{i j}\right) \hat{\mathbf{n}}_{i j}, \\
\mathbf{v}_{i j}^{t} & =\mathbf{v}_{i j}-\left(\mathbf{v}_{i j} \cdot \hat{\mathbf{n}}_{i j}\right) \hat{\mathbf{n}}_{i j}+\boldsymbol{\omega}_{i} \times \mathbf{b}_{i j}-\boldsymbol{\omega}_{j} \times \mathbf{b}_{j i} .
\end{aligned}
$$

The tangential displacement $\boldsymbol{\delta}_{i j}^{t}$ can be evaluated as [12]

$$
\begin{aligned}
\frac{d}{d t} \boldsymbol{\delta}_{i j}^{t} & =\mathbf{v}_{i j}^{t}-\frac{\mathbf{r}_{i j}}{r_{i j}^{2}}\left(\boldsymbol{\delta}_{i j}^{t} \cdot \mathbf{v}_{i j}\right) \\
\boldsymbol{\delta}_{i j}^{t}\left(t_{0}\right) & =0,
\end{aligned}
$$

which is integrated with the first order forward Euler method [7], starting from the initial time of contact $t_{0}$.

\section{C.2. Contact LaW}

There are many different contact laws to evaluate the forces between colliding particles. Here, the normal and tangential forces are modeled with linear elastic and linear dissipative contributions $[13,14]$. The contact law does not strongly affect the macroscopic friction coefficient [15], which justifies choosing the simple linear contact model. The total contact force on particle $i$ due to particle $j$ is then given by $\mathbf{f}_{i j}=\mathbf{f}_{i j}^{n}+\mathbf{f}_{i j}^{t}$, where the normal and tangential forces are given by

$$
\begin{aligned}
& \mathbf{f}_{i j}^{n}=k^{n} \delta_{i j}^{n} \hat{\mathbf{n}}_{i j}-\gamma^{n} \mathbf{v}_{i j}^{n}, \\
& \mathbf{f}_{i j}^{t}=-k^{t} \boldsymbol{\delta}_{i j}^{t}-\gamma^{t} \mathbf{v}_{i j}^{t},
\end{aligned}
$$

with normal and tangential spring constants $k^{n}$ and $k^{t}$, and damping constants $\gamma^{n}$ and $\gamma^{t}$ respectively.

The contact time $t_{c}$ for a central collision can be related to the contact properties as

$$
t_{c}=\pi / \sqrt{\frac{k^{n}}{m_{i j}}-\left(\frac{\gamma^{n}}{2 m_{i j}}\right)^{2}},
$$

where the reduced mass is given by $m_{i j}=m_{i} m_{j} /\left(m_{i}+m_{j}\right)$. The restitution coefficient $\epsilon$ is given by

$$
\epsilon=\exp \left(-t_{c} \gamma^{n} / 2 m_{i j}\right)
$$

It should be noted, that our definition of $\gamma^{n}$ differs with a factor two from the definition of Silbert et al. [16], but is in line with the definitions of Luding [14] and Weinhart et al. [12]. 
The contact-stiffness and damping are chosen such that the restitution coefficient, which is a material property, is the same for all types of collisions,

$$
\epsilon^{L L}=\epsilon^{S S}=\epsilon^{L S},
$$

where the superscripts $L L, S S$ and $L S$ denote large-large, small-small and large-small contacts respectively. Furthermore, the ratio between collision time and gravitational time is kept constant, which means that

$$
\frac{t_{c}^{L L}}{\sqrt{d^{L} / g}}=\frac{t_{c}^{S S}}{\sqrt{d^{S} / g}}=\frac{t_{c}^{L S}}{\sqrt{\left(d^{L}+d^{S}\right) / 2 g}} .
$$

For each type of contact, the tangential stiffness is related to the normal stiffness as $k^{t}=2 / 7 k^{n}$ and the the tangential damping coefficient equals the normal damping coefficient.

Furthermore, Coulomb friction on the contact level has been assumed, which means that the magnitude of $\boldsymbol{\delta}_{i j}^{t}$ is capped if necessary such that $\left|\mathbf{f}_{i j}^{t}\right| /\left|\mathbf{f}_{i j}^{n}\right| \leq \mu^{p}$. For this study, the value of $\mu^{p}$ depends on the size of the particles, where it is larger for large particles than for small particles. The relevant parameter values can be found in table 3.C.1. For a more detailed description of this contact law we refer to [12].

The total force $\mathbf{F}_{i}$ and torque $\mathbf{q}_{i}$ acting on particle $i$ are now given by

$$
\begin{aligned}
& \mathbf{F}_{i}=m_{i} \mathbf{g}+\sum_{j \neq i} \mathbf{f}_{i j}, \\
& \mathbf{q}_{i}=\sum_{j \neq i} \mathbf{b}_{i j} \times \mathbf{f}_{i j},
\end{aligned}
$$

where $\mathbf{g}$ is the gravitational vector, see figure 3.2 .

\section{C.3. DYNAMics}

The dynamics of a particle $i$ with mass $m_{i}$ and moment of inertia $I_{i}$ can now be described by

$$
\begin{aligned}
m_{i} \frac{\mathrm{d}^{2} \mathbf{r}_{i}}{\mathrm{~d} t^{2}} & =\mathbf{F}_{i}, \\
I_{i} \frac{\mathrm{d} \boldsymbol{\omega}_{i}}{\mathrm{~d} t} & =\mathbf{q}_{i} .
\end{aligned}
$$

These equations of motion are integrated with a second order velocity Verlet (leap-frog) scheme [17] to compute the position, velocity and angular velocity of each particle at each time step.

All necessary parameters for the discrete particle simulations are given in table 3.C.1, non-dimensionalised such that for a large particle $d^{L}=1, m^{L}=1$ and $g=1$. From these expressions, all properties for collisions between small particles and mixed collisions can be derived, see the thesis of Voortwis [18] for details. 


\begin{tabular}{|c|c|l|}
\hline parameter & value & description \\
\hline$g$ & 1 & gravitational constant \\
$d^{L}$ & 1 & large particle diameter \\
$d^{S}$ & $\sqrt[3]{1 / 2} \approx 0.79$ & small particle diameter \\
$\rho^{p}$ & $6 / \pi$ & particle density $\left(m^{L}=1, m^{S}=0.5\right)$ \\
$k^{n, L}$ & $2 \cdot 10^{5}$ & normal spring constant large particles \\
$k^{t, L}$ & $\frac{2}{7} k^{n, L}$ & tangential spring constant large particles \\
$\gamma^{n, L}$ & 25 & normal damping coefficient large particles \\
$\gamma^{t, L}$ & $\gamma^{n, L}$ & tangential damping coefficient large particles \\
$\mu^{p, L L}$ & 0.5 & microscopic particle friction large-large contacts \\
$\mu^{p, S S}$ & 0.25 & microscopic particle friction small-small contacts \\
$\mu^{p, L S}$ & 0.375 & microscopic particle friction large-small contacts \\
$\Delta t$ & $10^{-4}\left(\approx t_{c}^{L L} / 50\right)$ & time step for time integration schemes \\
\hline
\end{tabular}

Table 3.C.1: Non-dimensionalised parameter values used for the particle simulations

\section{C.4. Chute geometry}

For all simulations, the base of the flow consists of a few layers of fixed large particles, to imitate a rough surface. To determine the friction parameters for the continuum model, a short periodic chute of size $(x, y) \in[0,20] \times[0,10]$ is constructed such that it tilts in $x$ direction. It is filled with 1000 large particles and 2000 small particles, so that the volume fraction of each is the same. For a description of the algorithm to get the closure relations from a short periodic chute flow, we refer the interested reader to [12]. For the long chute simulations, the base of the short periodic chute is copied in $x$-direction to a total length of 2000, while keeping the width in $y$-direction constant. The inflow is regulated through a maser boundary, see section 3.C.5 for details.

\section{C.5. MASER INFLOW BOUNDARY CONDITION}

For the long chute simulations, the inflow must be non-accelerating and segregated in order to compare them to the continuum model. One of the options is to do this with a hopper with a large reservoir of particles. However, this means that at all times, the positions and velocities of all the particles that will ever appear in the simulation have to be computed. Furthermore, it can neither be assumed that a flow from a hopper is already segregated at the inflow of the chute, nor that the mass fraction is constant over time, which makes comparison with continuum models more difficult.

As an alternative, we designed a maser inflow boundary, which generates a fully developed inflow for a chute. Initially, it only consists of a periodic box for $(x, y) \in[-20,0] \times$ $[0,10]$. After the periodic flow reaches a steady state, the maser boundary starts generating particles: for each particle that passes the line $x=0$, the particle is both moved back to $x=-20$ as in a normal periodic boundary, and copied as inflow particle to its original position at the right, becoming an inflowing particle for the chute, see figure 3.C.1. The particles around $x=-20$ do not exert any forces on the particles around $x=0$; otherwise, the particles around $x=0$ would experience similar forces twice. However, the particles at $x=0$ do exert forces at the particles around $x=-20$ by the means of ghost- 


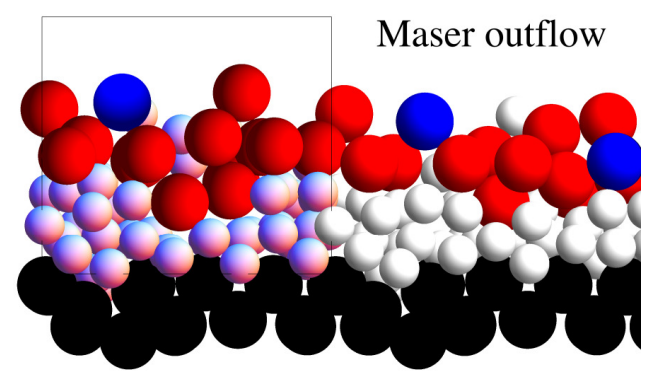

Figure 3.C.1: Schematic overview of a maser inflow-boundary. On the left, there is a periodic box with coloured particles to develop the flow. Once the flow is developed, the moving particles crossing the right periodic boundary are copied as an inflow particle onto the long chute.

particles. This way, a fully developed inflow with nearly constant height is realised. It should be noted, that the velocity inside the maser boundary is influenced by the velocity in the outflow domain, since those particles do exert forces on particles inside the maser boundary. Especially for subcritical flows, this influence can be substantial.

\section{REFERENCES}

[1] A. Harten, P. D. Lax, and B. v. Leer, On upstream differencing and Godunov-type schemes for hyperbolic conservation laws, SIAM review 25, 35 (1983).

[2] E. F. Toro, Riemann solvers and numerical methods for fluid dynamics: a practical introduction (Springer Science \& Business Media, 2013).

[3] G. Kesserwani, R. Ghostine, J. Vazquez, A. Ghenaim, and R. Mosé, Application of a second-order Runge-Kutta discontinuous Galerkin scheme for the shallow water equations with source terms, International journal for numerical methods in fluids 56, 805 (2008).

[4] B. Cockburn and C. W. Shu, TVB Runge-Kutta local projection discontinuous Galerkin finite element method for conservation laws. II. General framework, Mathematics of computation 52, 411 (1989).

[5] S. Bunya, E. J. Kubatko, J. J. Westerink, and C. Dawson, A wetting and drying treatment for the Runge-Kutta discontinuous Galerkin solution to the shallow water equations, Computer Methods in Applied Mechanics and Engineering 198, 1548 (2009).

[6] G. Saingier, S. Deboeuf, and P.-Y. Lagrée, On the front shape of an inertial granular flow down a rough incline, Physics of Fluids 28, 053302 (2016).

[7] W. H. Press, Numerical recipes 3rd edition: The art of scientific computing (Cambridge university press, 2007). 
[8] A. R. Thornton, D. Krijgsman, A. Te Voortwis, V. Ogarko, S. Luding, R. Fransen, S. Gonzalez, O. Bokhove, O. Imole, and T. Weinhart, A review of recent work on the discrete particle method at the University of Twente: An introduction to the opensource package MercuryDPM, DEM 6: Proc. 6th Int. Conf. , 393 (2013).

[9] A. R. Thornton, D. Krijsgman, R. Fransen, S. Gonzalez, D. R. Tunuguntla, A. Te Voortwis, S. Luding, O. Bokhove, and T. Weinhart, MercuryDPM: Fast particle simulations in complex geometries, News. Enginesoft 1, 48 (2013).

[10] T. Weinhart, D. R. Tunuguntla, M. P. Van Schrojenstein-Lantman, A. J. Van der Horn, I. F. C. Denissen, C. R. Windows-Yule, A. C. De Jong, and A. R. Thornton, MercuryDPM: A fast and flexible particle solver part A: Technical advances, in Proceedings of the 7th International Conference on Discrete Element Methods (Springer Singapore, Singapore, 2017) pp. 1353-1360.

[11] A. R. Thornton, T. Weinhart, S. Luding, and O. Bokhove, Modeling of particle size segregation: calibration using the discrete particle method, International Journal of Modern Physics C 23, 1240014 (2012).

[12] T. Weinhart, A. R. Thornton, S. Luding, and O. Bokhove, Closure relations for shallow granular flows from particle simulations, Granular Matter 14, 531 (2012).

[13] P. A. Cundall and O. D. L. Strack, A discrete numerical model for granular assemblies, Geotechnique 29, 47 (1979).

[14] S. Luding, Cohesive, frictional powders: contact models for tension, Granular matter 10, 235 (2008).

[15] A. R. Thornton, T. Weinhart, S. Luding, and O. Bokhove, Frictional dependence of shallow-granular flows from discrete particle simulations, The European Physical Journal E 35, 1 (2012).

[16] L. E. Silbert, D. Ertaş, G. S. Grest, T. C. Halsey, D. Levine, and S. J. Plimpton, Granular flow down an inclined plane: Bagnold scaling and rheology, Physical Review E 64, 051302 (2001).

[17] M. P. Allen and D. J. Tildesley, Computer simulation of liquids (Oxford university press, 1989).

[18] A. T. Voortwis, Closure laws for granular, shallow-layer, bi-disperse flows down an inclined chute, Master's thesis, MSM Group, Univ. Twente, the Netherlands (2013). 


\section{4}

\section{ANISOTROPIC COARSE GRAINING FOR SHALLOW FLOWS}

\subsection{INTRODUCTION}

Shallow particulate flows are a common natural phenomenon, for example in the form of landslides and debris-flows. Experimental study of these flows is costly and timeconsuming; furthermore, the flow properties that can be measured are limited [1]. Instead, computer simulations can be used to study these flows. Discrete particle simulations provide a lot of detailed data, such as not only the positions and velocities of all particles, but also e.g. the forces on each particle [2].

The main drawback of discrete particle simulations is that they are expensive in terms of computation time, as the position and velocity of each particle must be updated each time-step, and large-scale granular flows can easily have trillions of particles [3]. A better approach for these large-scale flows is to develop continuum models that predict the most important features of these flows, for example their height, velocity or concentration of each particle-type. Many continuum models have been developed, see [4] for a recent review of models for shallow granular flows over inclined planes that include segregation effects. Alternatively, one could develop multiscale methods that combine discrete particle simulations with continuum models, where for example the discrete particle simulations are used to obtain local closure relations for the continuum model (see e.g. [5, 6]).

For both the validation of continuum models and development of hybrid models, the results of discrete particle simulations must be somehow averaged in order to obtain continuum fields of the desired quantities. For an overview of techniques to go from particle-data to continuum quantities, we refer the interested reader to $[7,8]$ and references therein. Here, we use the coarse graining method [9-11], which smooths out the properties of each particle over a region centered around the particle. For a given set of points in time and space, the continuum fields can then be computed by summing over the contributions of all particles at these points. The advantages of coarse-graining are 
that (i) the resulting fields for mass, momentum and stress exactly satisfy the continuum equations for mass- and momentum-conservation everywhere, (ii) the resolution of the continuum fields is independent of the number of particles that is averaged over, (iii) the particles are neither be assumed rigid or spherical and (iv) the results are even valid for a single particle; no averaging over groups of particles is necessary [9-11].

In section (4.2) it is shown that while the standard coarse-graining method applied to long, shallow granular flows over inclined planes gives reasonable continuum fields for this system, these fields contain a lot of undesired fluctuations in the downstream direction. We therefore develop a tool that can coarse-grain discrete particle simulations in an anisotropic way, without losing any of the advantages of coarse graining listed above.

This chapter is structured as follows: first, we describe the simulation setup for our model system and show that the isotropic coarse-graining method gives suboptimal data. We then introduce our new anisotropic coarse-graining method and show that this method works well for the shallow granular flows that we are interested in. Finally we apply our anisotropic coarse-graining method to study the behaviour of particleconcentration in bidisperse granular flows over an inclined plane.

\subsection{METHOD}

\subsubsection{DisCRETE PARTICLE SIMULATION SETUP}

For the discrete particle simulations in this chapter, we use MercuryDPM [10, 12-14] to simulate a bidisperse mixture of particles with mean diameter $\bar{d}=\left(d^{L}+d^{S}\right) / 2=1$ and a certain size-ratio $S=d^{L} / d^{S}$, which is the ratio of diameters of the large and small particles respectively. The Coulomb friction-coefficient for large and small particles equals $\mu^{L}=0.5$ and $\mu^{S}=0.25$ respectively; the large particles are 'rougher' than small particles. All particles have the same density $\rho=\pi / 6$, such that a particle with diameter $d=1$ has mass $m=1$. Furthermore, the system is non-dimensionalised such that the magnitude of gravity $g=1$, and gravity has direction $(\sin \theta, 0,-\cos \theta)$, where $\theta$ is the angle of the inclined plane. For the particle-particle contacts, we use a linear spring-dashpot contact-model in which for all interactions the restitution coefficient equals $r_{c}=0.88$ and contact-time equals $t_{c}=0.005$, see $[8,15]$ for more details about the contact model. Since the restitution coefficient and contact-time depend on the radii of the particles, this means that we use three different particle-types, namely for the small, large and fixed bottom particles respectively.

Concerning the geometry, we consider an inclined plane with inclination angle $\theta=$ $23^{\circ}$ to the horizontal. The coordinate system $(x, y, z)$ is defined such that the $x$-axis points downslope, the $y$-axis across the slope and the $z$-axis is normal to the plane $[8,16]$. The flow is confined at the bottom by disordered fixed particles with diameter $d^{B}=1$ at $z \approx 0$, which is constructed in the same way as in the simulations of Weinhart et al. [8]. In $y$-direction, periodic boundary conditions are imposed at $y=0$ and $y=6$. In the region $0<x \leq 20$ we have a maser-inflow boundary, which first acts as a periodic boundary until the flow is steady in terms of density, velocity and small-particle concentration $\phi$. Then it is activated, so that every particle that crosses the boundary at $x=20$, is both let through to the outflow domain, and copied to its corresponding position around $x=0$, see [16] for more details. For each simulation, the maser-inflow boundary contains the 


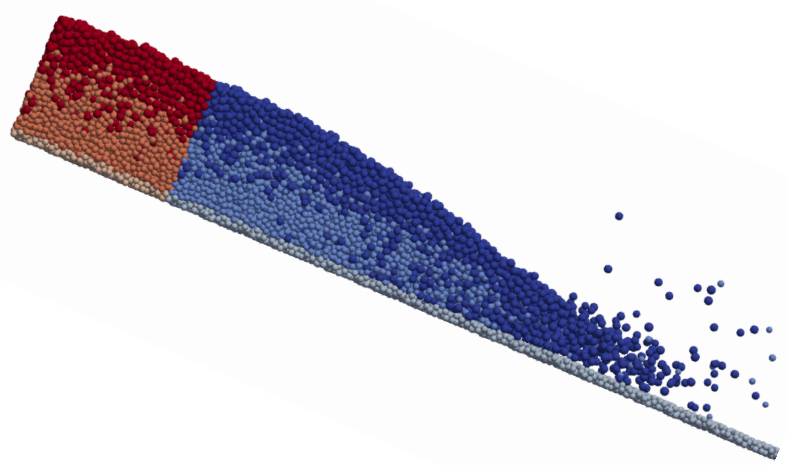

Figure 4.1: Example of the geometry of bidisperse long-chute simulations: there is a rough bottom made of fixed particles, a maser inflow-boundary on the left (red/orange particles) and the outflow domain on the right (blue particles). Note, that the flow is fully developed before the maser-boundary is activated, so that the outflow domain has steady inflow conditions.

same volume of small particles and large particles. An example of the geometry is given in figure 4.1. All simulations are run in parallel on 36 cores, where the domain is divided in equal parts in the $x$-direction.

\subsubsection{ANISOTROPIC COARSE-GRAINING}

In order to extract continuum data from discrete particle simulations, the coarse-graining method for bidisperse flows [9-11] is a logical choice, since the resulting fields conserve mass and momentum. The basic idea is to use a coarse-graining function $\mathbb{W}\left(\mathbf{r}-\mathbf{r}_{i}\right)$ to distribute properties such as density and momentum of a particle over a certain volume. Define $\hat{\mathbf{r}}=\hat{\mathbf{r}}(\mathbf{r}, t)=\mathbf{r}-\mathbf{r}_{i}(t)$ for brevity of notation. Trivially, $\mathbf{r} \in \mathbb{R}^{d} \Longleftrightarrow \hat{\mathbf{r}} \in \mathbb{R}^{d}$ and $\mathbf{r}, \hat{\mathbf{r}}$ have the same range, namely $\mathbb{R}^{d}$. We now define a coarse-graining function as follows:

Definition 1. $W$ is a coarse-graining function over $\mathbb{R}^{d}$ if it has the following properties:

1. $\mathbb{W}(\hat{\mathbf{r}}) \geq 0 \forall \hat{\mathbf{r}} \in \mathbb{R}^{d}$ (non-negativity),

2. $\mathbb{W} \in C^{1}$ (differentiability),

3. $\int_{\mathbb{R}^{d}} \mathbb{W}(\hat{\mathbf{r}}) d \hat{\mathbf{r}}=1$ (unity),

4. $W(\hat{\mathbf{r}})=\mathbb{W}(-\hat{\mathbf{r}})$ (symmetry).

Coarse-graining functions are often isotropic, i.e. $\mathbb{W}(\hat{\mathbf{r}})=W_{i}(|\hat{\mathbf{r}}|)$ for a function $\mathbb{W}_{i}$ with scalar input. Furthermore, instead of using a single coarse-graining function, one often looks at a family of coarse-graining functions with a coarse-graining width $w$ as 


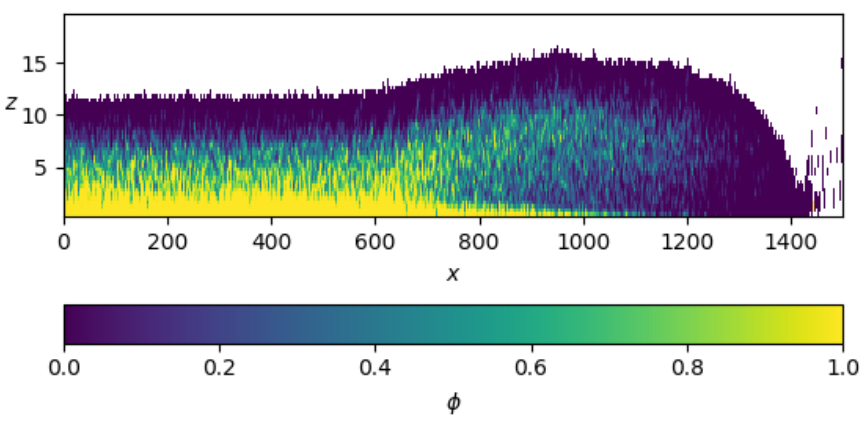

Figure 4.2: Coarse-grained small-particle concentration of a shallow, bidisperse granular flow. The coarsegraining function is isotropic in $x$ and $z$, with a coarse-graining width of $w=\bar{d}$. Note the difference in scale in $x$ and $z$.

an explicit parameter, and calibrates this parameter depending on the system and desired continuum description. Examples of families of coarse-graining functions include Heaviside-functions, Gaussian-distributions and Lucy-polynomials [11].

If we coarse-grain the flow with an isotropic coarse-graining function, the field shows many fluctuations in the $x$-direction, see figure 4.2. This is because the isotropic coarse graining method does not take into account the shallowness of the large particulate geophysical flows that we are interested in. This shallowness should be reflected in the continuum fields corresponding to these flows, where the changes in the $x$-direction should be much slower than in the $z$-direction [17]. Therefore, we propose an anisotropic coarse-graining method, where we use

$$
\tilde{W}(\hat{\mathbf{r}})=\operatorname{det}(A) \mathscr{W}(A \hat{\mathbf{r}}),
$$

as our coarse-graining function, where $A \in \mathbb{R}^{d \times d}$ is a constant, positive definite matrix and $W$ is a (isotropic) coarse-graining function. If $A$ is taken as the identity matrix, (4.1) reduces back to the normal isotropic function definition.

Theorem 1. $\tilde{W}$ is a coarse-graining function if $W$ is a coarse-graining function

Proof. In order to proof that $\tilde{W}$ is a coarse-graining function, we show that it satisfies all properties of definition 1:

1. (Positivity) Define $\mathbf{w}=A \hat{\mathbf{r}} \in \mathbb{R}^{d}$ and note that $\operatorname{det}(A)>0$ since $A$ is positive definite. Then for any $\hat{\mathbf{r}} \in \mathbb{R}^{d}, \tilde{W}(\hat{\mathbf{r}})=\operatorname{det}(A) \mathbb{W}(A \hat{\mathbf{r}})=\operatorname{det}(A) \mathscr{W}(\mathbf{w}) \geq 0$.

2. (Differentiability) Since $\mathbb{W}(\hat{\mathbf{r}}) \in C^{1}$ and $A \hat{\mathbf{r}}$ is a continuously differentiable function in $\hat{\mathbf{r}}, \mathbb{W}(A \hat{\mathbf{r}}) \in C^{1}$. Moreover, $\operatorname{det}(A)$ is a constant, so $\tilde{W}(\hat{\mathbf{r}})=\operatorname{det}(A) \mathscr{W}(A \hat{\mathbf{r}}) \in C^{1}$.

3. (Unity) Using the change of variables $\mathbf{w}=A \hat{\mathbf{r}}$, with Jacobian $\operatorname{det}(A)$, the integral of $\tilde{W}$ 
can be reduced to

$$
\begin{aligned}
\int_{\mathbb{R}^{d}} \tilde{W}(\hat{\mathbf{r}}) d \mathbf{r} & =\int_{\mathbb{R}^{d}} \operatorname{det}(A) \mathscr{W}(A \hat{\mathbf{r}}) d \mathbf{r}, \\
& =\int_{\mathbb{R}^{d}} \operatorname{det}(A) \mathbb{W}(\mathbf{w}) \frac{1}{\operatorname{det}(A)} d \mathbf{w}, \\
& =\int_{\mathbb{R}^{d}} \mathscr{W}(\mathbf{w}) d \mathbf{w} \\
& =1 .
\end{aligned}
$$

Note, that we used that $A$ is positive definite and therefore invertible to define the integration domain for $\mathbf{w}$.

4. (Symmetry) Realising $A(-\hat{\mathbf{r}})=-A \hat{\mathbf{r}}$, use the definition of $\tilde{W}$ to arrive at

$$
\begin{aligned}
\tilde{W}(-\hat{\mathbf{r}}) & =\operatorname{det}(A) \mathbb{W}(A(-\hat{\mathbf{r}})), \\
& =\operatorname{det}(A) \mathscr{W}(-(A \hat{\mathbf{r}})), \\
& =-\operatorname{det}(A) \mathscr{W}(A \hat{\mathbf{r}}), \\
& =-\tilde{W}(\hat{\mathbf{r}}) .
\end{aligned}
$$

With this definition of our coarse-graining function $\tilde{W}$, the eigenvalues $\lambda_{i}$ of $A$ can be seen as the multiplier for the coarse-graining widths in the directions of their corresponding eigenvectors. For example in the shallow granular flows we study here, we wish to have a coarse-graining function that is more elongated in the $x$-direction than in the $z$-direction. This corresponds to the anisotropy matrix

$$
A=\left[\begin{array}{ll}
\epsilon & 0 \\
0 & 1
\end{array}\right]
$$

where $0<\epsilon \ll 1$, and $\operatorname{det}(A)=\epsilon$.

Using the anisotropic coarse-graining function $\tilde{W}$, we define the density $\rho$ of the system at position $\mathbf{r}$ and time $t$ similar to [9-11]:

$$
\rho(\mathbf{r}, t)=\sum_{i=1}^{N_{f}} m_{i} \tilde{W}\left(\mathbf{r}-\mathbf{r}_{i}(t)\right),
$$

where $N_{f}$ is the number of flow particles and $m_{i}$ is the mass of particle $i$. The density can be computed for either the complete system, or for each type of particle separately [11]. In this work, we are interested in the small-particle concentration $\phi(\mathbf{r}, t)=\rho^{S}(\mathbf{r}, t) / \rho(\mathbf{r}, t)$, where $\rho^{S}$ is the partial density of the phase of small particles. Since we have proved that $\tilde{W}$ is a coarse-graining function, other continuum fields can be defined as in [9-11]. These works define the momentum and stress such that mass and momentum are conserved when using general coarse-graining functions $\mathbb{W}$. Moreover, all the other advantages of coarse-graining also apply to our anisotropic coarse-graining method because $\tilde{W}$ is a coarse-graining function.

In the next section, the principle of anisotropic coarse-graining is validated for a long, shallow bidisperse granular flow over an inclined plane. 


\subsubsection{VALIDATION FOR SHALLOW BIDISPERSE FLOWS}

Now that we have shown how to go from an isotropic coarse-graining function $\mathbb{W}$ to an anisotropic coarse-graining function $\tilde{W}$, we wish to find the correct widths $w_{z}=w$ and $w_{x}=w / \epsilon$ for the long bidisperse flows from an inclined plane that we are interested in, using the anisotropy matrix $A$ as given by (4.2).

In this chapter we use two-dimensional Lucy-polynomials [18] as our coarse-graining function,

$$
\mathscr{W}(\hat{\mathbf{r}})= \begin{cases}\frac{\pi}{5 w^{2}}\left(1-6\left(\frac{|\hat{\mathbf{r}}|}{w}\right)^{2}+8\left(\frac{|\hat{\mathbf{r}}|}{w}\right)^{3}-3\left(\frac{|\hat{\mathbf{r}}|}{w}\right)^{4}\right) & \text { if }|\hat{\mathbf{r}}|<w \\ 0 & \text { otherwise }\end{cases}
$$

It can easily be shown that $\mathbb{W}$, and thus also $\tilde{W}$, is a coarse-graining function over $\mathbf{r} \epsilon$ $\mathbb{R}^{2}$. It has compact support, and it is a polynomial, which are both advantageous for computation time compared to a more expensive Gaussian function and is therefore often used as a coarse-graining function (e.g. [11, 19]).

As an example, in this section we use a bidisperse flow with size-ratio $S=1.5$, inclination angle $\theta=23^{\circ}$ and inflow-height $h \approx 12$ at $t=1000$. A few examples of anisotropic coarse-graining applied to this flow are given in figure 4.3, where $w=1$ is chosen as the coarse-graining width in the $z$-direction, as this is a robust value for similar small-scale bidisperse flows [11]. The first plot shows the small-particle concentration $\phi$ of the flow for isotropic coarse-graining $(\epsilon=1)$; subsequent plots show $\phi$ for decreasing $\epsilon$. Note that the plots in figure 4.3 all use a very high resolution, but a lower resolution in the $x$-direction would not significantly change the plots with small $\epsilon$. Moreover, the coarsegraining method does not require a grid; if one would for example require the values of $\phi$ at the corners of an unstructured mesh, the method can deal with this effortlessly.

It is clear that one cannot just use any value of the anisotropy parameter $\epsilon<1$ here; too large and the field contains a lot of noise (e.g. figure 4.3(a)), too small and the interesting features are smoothed out (e.g. figure 4.3(d)). However, looking at figure 4.3(b) and (c), we see that the field of $\phi$ is very similar, even though $\epsilon$ differs by a factor five. This gives rise to the question of whether there is a range of values for $\epsilon$ for which the solution is insensitive to the exact value of $\epsilon$, i.e. if there is a plateau in the values $\phi$. Analogously to [11], we choose a few interesting points within the flow, and compare the values of $\phi$ at these points for various $\epsilon$. Since in this case the interesting behaviour takes place in the region $800<x<1200$, we focus only on the value of $\phi$ in this region. The results can be seen in figure 4.4. We see that there is indeed a plateau in $\epsilon$ for this flow for approximately $30<1 / \epsilon<150$.

Going one step further, we vary the coarse-graining widths $w_{x}=w / \epsilon$ and $w_{z}=w$ independently, in analogy to what was done in [11] for time and space coarse-graining. In figures 4.3 and 4.4 , we see that the point $x=800, z=6$ is both close to the centre of the breaking size-segregation wave and is representative for the region where the value of $\phi$ is sensitive to $\epsilon$, so we take this point for determining suitable values for $w_{x}$ and $w_{z}$. We let $w_{x}$ go from 0.1 to 1000 on a logarithmic scale in 100 steps, and let $w_{z}$ go from 0.1 to 10 in 50 steps.

The results for the value of $\phi$ at this point can be found in figure 4.5(a). We see that there is a plateau for which the value of $\phi$ is not very sensitive on the coarse-graining 

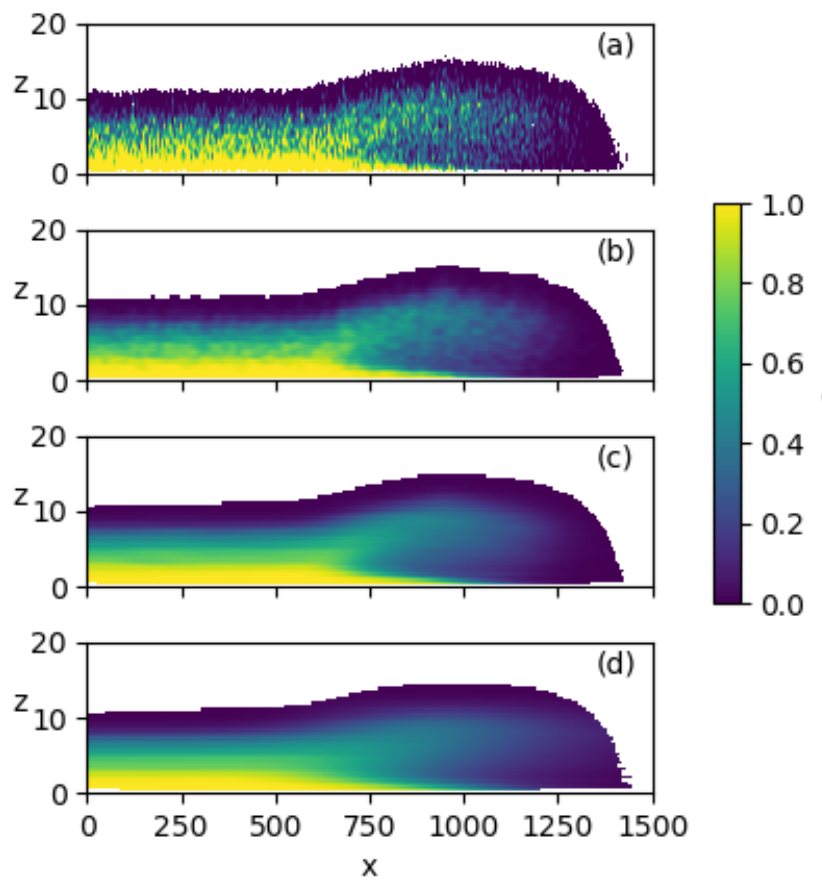

Figure 4.3: Small-particle concentration profiles for a bidisperse flow with various levels of smoothing. (a) No smoothing $(\epsilon=1)$ : the field contains lots of unwanted fluctuations. (b) $\epsilon=1 / 30$ and (c) $\epsilon=1 / 150$ : smoothing on either end of the plateau. Between these values, the field is not very sensitive to the $\epsilon$. (d) $\epsilon=1 / 500$ : oversmoothing, the shape of the breaking segregation wave is barely visible anymore.

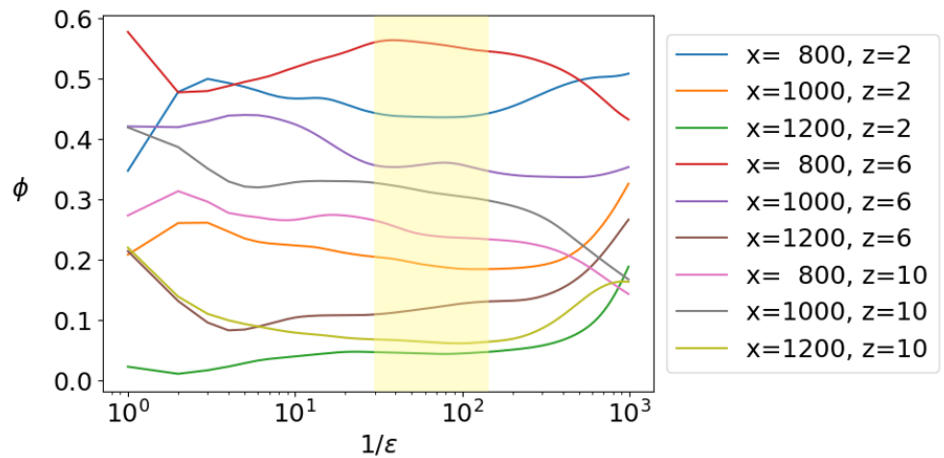

Figure 4.4: Relation between the anisotropy parameter $\epsilon$ and the small-particle concentration $\phi$ at given points for a flow of height 12 and with size-ratio $S=1.5$ at $t=1000$. We see a plateau in $\phi$ between $1 / \epsilon \approx 30$ and $1 / \epsilon \approx 150$, which means that the continuum field of $\phi$ is not very sensitive to $\epsilon$ in this range. 

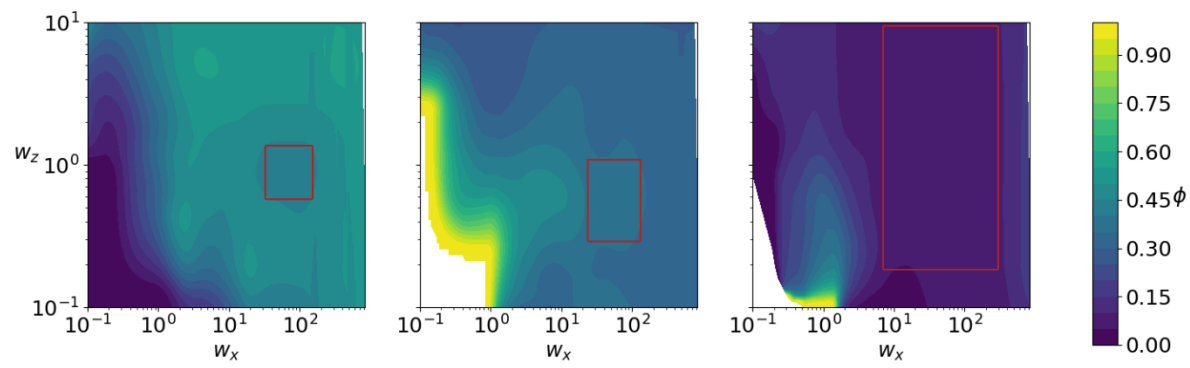

Figure 4.5: Value of $\phi$ at the points (a) $(800,6),(\mathrm{b})(1000,6)$ and (c) $(1200,6)$ at various values of $w_{x}$ and $w_{z}$. The plateaus in small-particle concentration are denoted by red rectangles, which have different sizes and positions for different $(x, z)$ points in the flow. The white region indicates that for these widths, there is no particle centre close enough to this point to contribute to the continuum fields. Note the logarithmic scale in both directions.

width $w$ and anisotropy parameter $\epsilon$, here denoted by the red rectangle. We validate that this feature is not unique to the point $(x, z)=(800,6)$ by also looking at some other points, for example $(x, z)=(1000,6)$ and $(x, z)=(1200,6)$, which are also inside the interesting region of the breaking segregation wave. We see that both these plots have a plateau for the small-particle concentration as well, which are somewhat different from the plateau for $(x, z)=(800,6)$. However, the region $w_{x} \in(30,150)$ and $w_{z} \in(0.6,1.5)$ is in a plateau for all of these points. We are therefore confident that coarse-graining widths taken within this plateau result in continuum fields that are independent of the coarsegraining width. The fact that the plateau is the region $w_{x} \in(30,150)$ and $w_{z} \in(0.6,1.5)$, is interesting for mainly two reasons: (i) it shows that the choice $w_{z}=1$ that is obtained from small-scale flows with periodic boundaries [11], is still a good choice for this larger system, and (ii) there is no point $w_{x}=w_{z}$ in this region; there is no coarse-graining width for which standard isotropic coarse graining gives a small particle concentration that is independent of that width. This confirms again that in this system, where we use information of only one time-step, anisotropic coarse-graining is required for reliable continuum fields. Armed with this information, we apply this smoothing method to the long-chute flows that we are interested in.

\subsection{APPLICATION: SEGREGATION BEHAVIOUR IN BIDISPERSE SHALLOW GRANULAR FLOWS}

Now, we apply the smoothing technique of the last section to shallow, bidisperse granular flows with various size-ratios and inflow-heights. For all of these flows, we determined that the coarse-graining widths of $w_{z}=1, w_{x}=50$ are robust values. The smoothed small-particle concentration for flows with an inflow-height $h \approx 6$ can be found in figure 4.6. Since one particle-type has a larger microscopic friction coefficient $\mu$, there is segregation in all of these flows, even in the mixture where the sizes of both particle-types are the same. This is consistent with [20,21], who saw segregation based on microscopic friction in granular flows in other geometries. Note that in this case, this 

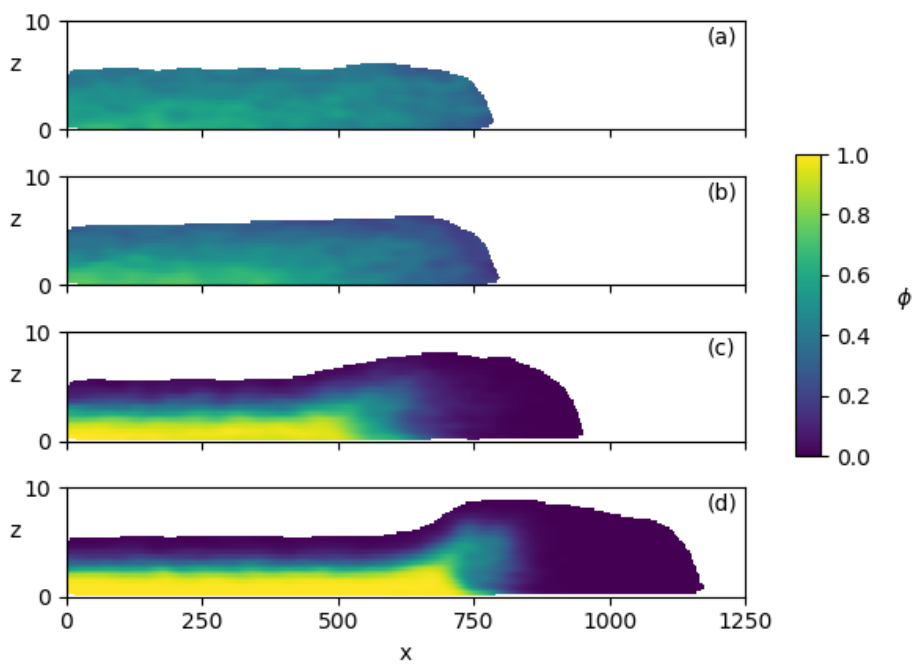

Figure 4.6: Small particle-concentration $\phi$ for different mixtures with inflow-height $h \approx 6$ at $t=3000$ : (a) $S=1$, (b) $S=1.025$, (c) $S=1.25$, (d) $S=1.5$. For all mixtures, the average particle diameter equals $\bar{d}=1$ and the large particles are more frictional than the small particles.

effect is so subtle that it might easily be overlooked if the data was not smoothed out in the $x$-direction.

Considering the segregation structure, all flows show a higher concentration of large, frictional particles at the front compared to the inflow-region. This causes the flow to dissipate more energy in the front, and therefore the flow is deeper near the front than at the inflow; a bulbous head has developed [16, 22]. This effect gets stronger when the size-ratio, and therefore the segregation rate, increases.

For the higher size-ratios, $S=1.25$ and $S=1.5$, we observe a breaking segregation wave [23-26] where the large particles that are deposited in the front, are taken up by the rest of the flow and segregate upwards again. When $S=1.5$, it is more compact and the segregation-profile is sharper than for $S=1.25$; in the latter case, the breaking segregation wave is more smoothed out. It is interesting that this kind of waves are observed in a flow that is only six average particle-diameters thick and has a size-ratio of only 1.25. This implies that it is a very robust phenomenon in shallow segregating flows.

When looking at flows of the same bidisperse mixtures, but with a greater inflowheight, we see similar patterns, see figure 4.7. Note, that these flows are now plotted at $t=1000$ instead of $t=3000$. Again, the mixtures with equal-sized and almost equalsized particles segregate very weakly and show now discernible breaking segregation wave and no clear bulbous head. The mixtures with $S=1.25$ and $S=1.5$ on the other hand, show both a bulbous head and a breaking segregation wave. The segregation is not as sharp here as with the shallower flows, which is caused by the higher downstream velocity of the flow [27]. Furthermore, we observe that the breaking segregation wave 

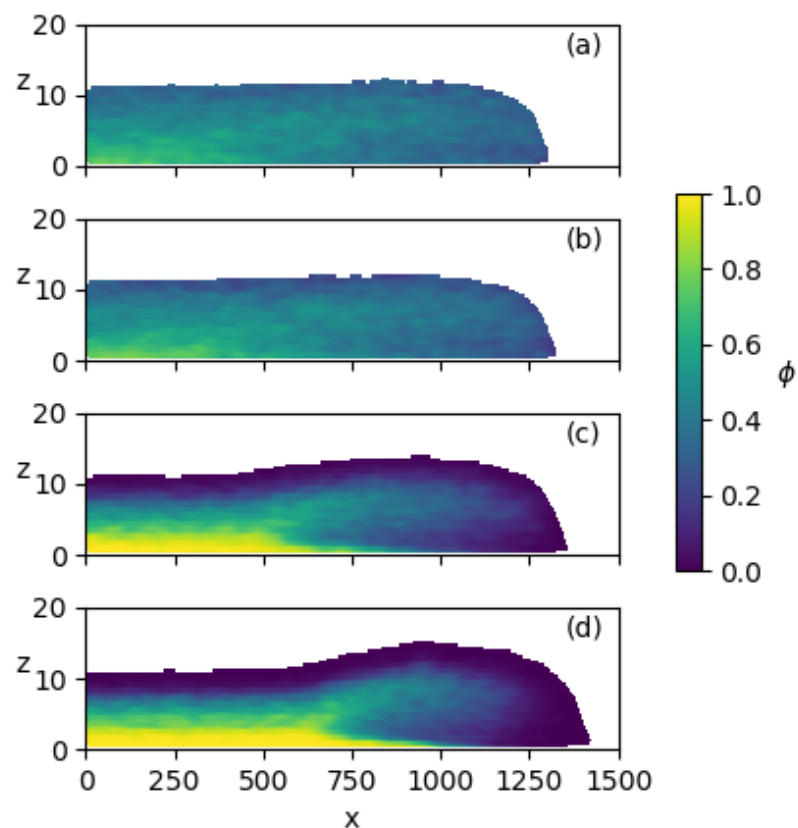

Figure 4.7: Small particle-concentration $\phi$ for different mixtures with inflow-height $h \approx 12$ at $t=1000$ : (a) $S=1$, (b) $S=1.025$, (c) $S=1.25$, (d) $S=1.5$. For all mixtures, the average particle diameter equals $\bar{d}=1$ and the large particles are more frictional than the small particles.

is more elongated here than for the flows with a lower inflow-height in figure 4.6. This is in agreement with the theory of Thornton and Gray [23], which states that the length of the breaking segregation wave scales quadratically with inflow-height. Similar to the very shallow flows of figure 4.6 , the breaking segregation wave is more elongated in the $S=1.25$ case than in the $S=1.5$ case, although it is much more diffuse in the former, which is also in agreement with [23].

The asymmetry of the breaking segregation wave, which is predicted by Gajjar et al. [25], van der Vaart et al. [26], is clearly visible in e.g. figure 4.7(d), where there is a thin region of large particles penetrating the small-particle region in the bottom $(750<x<$ 1200 ), while a thicker layer of a mixture of small and large particles lies on top of that. Unlike $[25,26]$, there is no region of pure small particles in our breaking segregation wave. This is because in $[25,26]$, there was an upstream region of pure small particles, which is missing from our simulations. It should therefore be noted that shallow flows on a conveyor belt might not be the ideal system to mimic a long flows from fixed inclined planes, and caution is advised when generalising results from conveyor belts to fixed inclined planes. 


\subsection{Conclusion AND OUTLOOK}

In this work, we developed a coarse-graining tool that uses an anisotropic coarse-graining function, and can therefore capture anisotropic features of granular flows with a very limited amount of data. This method is applied to shallow bidisperse flows over an inclined plane, where we see that there is a range of coarse-graining widths in both the $x$ and $z$-directions where the coarse-grained fields are not very sensitive to these widths. Most noteworthy is that in this case, with data from only one time-step, the line $w_{x}=w_{z}$ is not in this range, and therefore we do actually need our anisotropic coarse-graining tool to get good continuum fields from our discrete particle simulations.

Our new anisotropic coarse-graining tool is applied to a variety of shallow bidisperse granular flows, where we observe that segregation is induced by both a difference in microscopic friction and a difference in particle-size. With a low segregation-rate, the profiles of the small-particle concentration is more or less monotonic in $x$ and $z$, while breaking segregation waves are observed for flows with a higher segregation rate. Furthermore, the width of the breaking segregation wave is dependent on the height of the flow and segregation rate, where the breaking segregation wave becomes narrower with increasing segregation rate and decreasing height. We note that the behaviour of our breaking segregation waves is different from the ones generated by $[25,26]$ on a conveyor belt due to a different flow composition upstream. Further research is necessary to understand the relation between breaking segregation waves on a conveyor belt and a fixed inclined plane, and to understand their influence on for example the run-out distance of a granular flow.

In order to make our anisotropic coarse-graining tool more practical, the choice of appropriate coarse-graining widths and directions should be automated, so that our tool can be used to provide data for continuum solvers in order to develop reliable multiscale simulation methods. Then it can be applied to a variety of anisotropic granular systems, from flows on inclined planes to silo flows and particle spreading in additive manufacturing.

\section{REFERENCES}

[1] R. M. Iverson, The physics of debris flows, Reviews of geophysics 35, 245 (1997).

[2] S. Luding, Introduction to discrete element methods: basic of contact force models and how to perform the micro-macro transition to continuum theory, European Journal of Environmental and Civil Engineering 12, 785 (2008).

[3] S. A. Dunning and P. J. Armitage, The grain-size distribution of rock-avalanche deposits: implications for natural dam stability, in Natural and artificial rockslide dams (Springer, 2011) pp. 479-498.

[4] J. M. N. T. Gray, Particle segregation in dense granular flows, Annual Review of Fluid Mechanics 50, 407 (2018).

[5] A. R. Thornton, T. Weinhart, V. Ogarko, and S. Luding, Multi-scale methods for multi-component granular materials, Comp. Meth. Mat. Sci 13, 197 (2013). 
[6] M. K. Borg, D. A. Lockerby, and J. M. Reese, A multiscale method for micro/nano flows of high aspect ratio, Journal of Computational Physics 233, 400 (2013).

[7] S. Luding and F. Alonso-Marroquín, The critical-state yield stress (termination locus) of adhesive powders from a single numerical experiment, Granular Matter 13, 109 (2011).

[8] T. Weinhart, A. R. Thornton, S. Luding, and O. Bokhove, Closure relations for shallow granular flows from particle simulations, Granular Matter 14, 531 (2012).

[9] I. Goldhirsch, Stress, stress asymmetry and couple stress: from discrete particles to continuous fields, Granular Matter 12, 239 (2010).

[10] T. Weinhart, A. R. Thornton, S. Luding, and O. Bokhove, From discrete particles to continuum fields near a boundary, Granular Matter 14, 289 (2012).

[11] D. R. Tunuguntla, A. R. Thornton, and T. Weinhart, From discrete elements to continuum fields: Extension to bidisperse systems, Computational particle mechanics 3, 349 (2016).

[12] MercuryDPM, https : //www . mercurdpm . org, last accessed on August 27th 2019.

[13] A. R. Thornton, T. Weinhart, S. Luding, and O. Bokhove, Modeling of particle size segregation: calibration using the discrete particle method, International Journal of Modern Physics C 23, 1240014 (2012).

[14] T. Weinhart, M. Post, I. F. C. Denissen, D. R. Tunuguntla, E. Grannonio, N. Losacco, J. Barbosa, W. K. Den Otter, and A. R. Thornton, Fast, flexible particle simulations: An introduction to MercuryDPM, in Proceedings of the 8th International Conference on Discrete Element Methods (2019).

[15] S. Luding, Cohesive, frictional powders: contact models for tension, Granular matter 10, 235 (2008).

[16] I. F. C. Denissen, T. Weinhart, A. Te Voortwis, S. Luding, J. M. N. T. Gray, and A. R. Thornton, Bulbous head formation in bidisperse shallow granular flow over an inclined plane, Journal of Fluid Mechanics 866, 263 (2019).

[17] C. G. Johnson, B. P. Kokelaar, R. M. Iverson, M. Logan, R. G. LaHusen, and J. M. N. T. Gray, Grain-size segregation and levee formation in geophysical mass flows, Journal of Geophysical Research: Earth Surface 117 (2012).

[18] L. B. Lucy, A numerical approach to the testing of the fission hypothesis, The astronomical journal 82, 1013 (1977).

[19] T. Weinhart, R. Hartkamp, A. R. Thornton, and S. Luding, Coarse-grained local and objective continuum description of three-dimensional granular flows down an inclined surface, Physics of Fluids 25, 070605 (2013).

[20] K. A. Gillemot, E. Somfai, and T. Börzsönyi, Shear-driven segregation of dry granular materials with different friction coefficients, Soft matter 13, 415 (2017). 
[21] K. Van der Vaart, M. P. Van Schrojenstein Lantman, T. Weinhart, S. Luding, C. Ancey, and A. R. Thornton, Segregation of large particles in dense granular flows suggests a granular saffman effect, Physical Review Fluids 3, 074303 (2018).

[22] B. P. Kokelaar, R. L. Graham, J. M. N. T. Gray, and J. W. Vallance, Fine-grained linings of leveed channels facilitate runout of granular flows, Earth and Planetary Science Letters 385, 172 (2014).

[23] A. R. Thornton and J. M. N. T. Gray, Breaking size segregation waves and particle recirculation in granular avalanches, Journal of Fluid Mechanics 596, 261 (2008).

[24] J. M. N. T. Gray and C. Ancey, Segregation, recirculation and deposition of coarse particles near two-dimensional avalanche fronts, Journal of Fluid Mechanics 629, 387 (2009).

[25] P. Gajjar, K. van der Vaart, A. R. Thornton, C. G. Johnson, C. Ancey, and J. M. N. T. Gray, Asymmetric breaking size-segregation waves in dense granular free-surface flows, Journal of Fluid Mechanics 794, 460 (2016).

[26] K. van der Vaart, A. R. Thornton, C. G. Johnson, T. Weinhart, L. Jing, P. Gajjar, J. M. N. T. Gray, and C. Ancey, Breaking size-segregation waves and mobility feedback in dense granular avalanches, Granular matter 20, 46 (2018).

[27] J. W. Vallance and S. B. Savage, Particle segregation in granular flows down chutes, in IUTAM Symposium on Segregation in Granular Flows (Springer, 2000) pp. 31-51. 


\section{5}

\section{SEGREGATION OF ELLIPSOIDAL}

\section{PARTICLES IN A ROTATING DRUM}

\subsection{INTRODUCTION}

Segregation in granular flows poses large challenges in many industrial processes. Though still poorly understood, much progress has been made in modelling segregation based on size and, to a lesser extent, density [1-3]. Other mechanisms of segregation, such as particle shape, have received even less scientific attention. This chapter seeks to study the influence of particle elongation on segregation in rotating drums.

Rotating drums are seen in many applications, and have proven to be an effective system for studying segregation in shear flows. Because it is a closed system and the number of particles can be relatively small, it is suitable for both experiments and discrete particle simulations. Depending on the Froude number and other non-dimensional parameters, the flow can be categorised as slipping, surging, rolling, cascading, cataracting or centrifuging [4-6], see figure 5.1. The most relevant regime for industrial applications is the rolling regime, where the flow consists of a thin shear flow at the surface, and a passive solid body rotation below. Another characteristic of the rolling regime is a flat surface, which changes to an s-shape in the cascading regime.

It has been shown by [7] that the transition between the rolling regime and cascading regime is dependent on the elongation of the particles: more elongated particles enter the cascading regime at a lower Froude number than less elongated particles. They also showed that the ellipsoidal particles have a preferred orientation, which is consistent with observations in shear cells [8-10] and flows over inclined planes (chute flows) [11].

He et al. [12] showed that in rotating drums filled with mixtures of spheres and ellipsoids of equal volume and density, the ellipsoids segregate towards the core for ellipsoids with a small aspect-ratio 2, while the ellipsoids segregate to the rim if the ellipsoids have a larger aspect-ratio 4 . Looking at spheres and more blocky superquadric particles with equal radii, Pereira and Cleary [13] found that the blocky particles segregate to the core, even though they have a larger volume than the spheres. They showed that this segrega- 


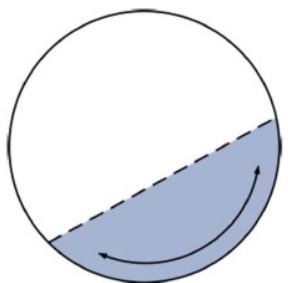

(a) Slipping or slumping

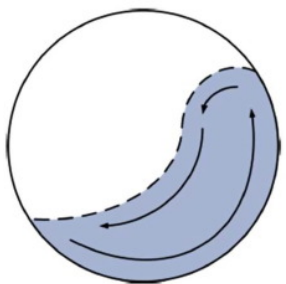

(d) Cascading

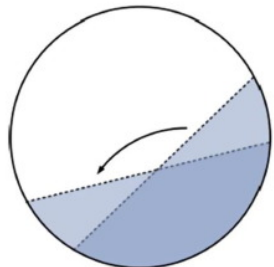

(b) Surging

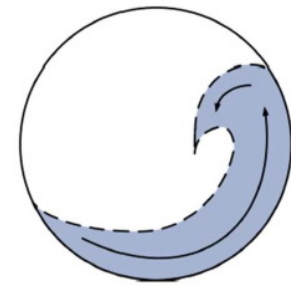

(e) Cataracting

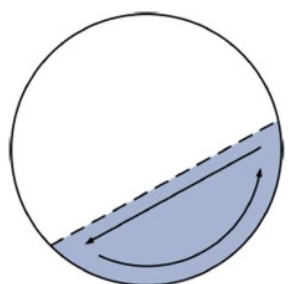

(c) Rolling

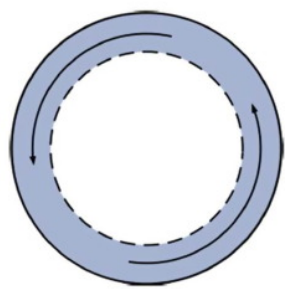

(f) Centrifuging

Figure 5.1: Graphical illustration of the various flow-regimes in rotating drums. Figure reproduced with permission of [6].

tion occurs along the surface of the flow, with the spheres having a much higher surface velocity than the blocky particles. They therefore postulate that the segregation occurs due to a difference in flowability, or rate of energy dissipation, of the particles, a hypothesis that has also been adopted by He et al. [12] for mixtures of spheres and ellipsoids with aspect-ratio 2.

If segregation indeed occurs along the flow surface, then studies that look at segregation in chute flows can be applied to segregation in rotating drums. In discrete particle simulations of chute flows with dumbbell-shaped particles (two connected spheres) of aspect ratios up to 1.4, Mandal and Khakhar [14] found that the segregation was only dependent on the geometric mean diameter of the particles. Using the geometric mean diameter, their simulations fit both the $\mu-I$ theory $[15,16]$ and segregation model of Gray and Chugunov [17]. The aspect ratios in [14] are much smaller than even the small aspect-ratio in [12], so they are useful for looking at the segregation behaviour at the surface of the flow for small aspect-ratios.

Looking at bounded heap-flows when using rods of different lengths, up to aspectratio 4, Zhao et al. [18] found that the segregation strength only increases with the aspectratio. They ascribe the segregation purely to percolation effects similarly to size-segregation in the same geometry. It is likely that the absence of inverse segregation is due to the different geometry, since the particles in a rotating drum have a location-dependent preferred orientation and hence the percolation behaviour can be vastly different. Another explanation would be the difference in volume, as they kept the cross-section of each cylinder the same and varied the length. In open heap flows with particles formed of two different irregularly clump formations, Alizadeh et al. [19] postulated that interlocking is also important for segregation, since it limits percolation. Their simulations show that segregation cannot be explained by just a difference in rolling friction, although that would explain part of the segregation, and hence the angle of repose is not the only rele- 
vant parameter in their system.

In this chapter, we study both the direction and strength of segregation based on particle elongation in a rotating drum. In particular, we want to extend the results of [12] by looking at intermediate aspect ratios, both to find the transition point, and to study whether the transition is smooth. For this, we use discrete particle simulations of mixtures of spheres and prolate ellipsoids in a rotating drum in the rolling regime. From there, coarse graining [20-22] is applied in order to obtain fields for the concentration of spheres in each part of the flow.

This chapter is organised as follows: in section 5.2, the simulation setup and analysis techniques are discussed. In section 5.3 we present the segregation profiles and mixing indices for mixtures of spheres and prolate ellipsoids. Lastly, section 5.4 summarises the conclusions of this chapter and gives an outlook for future work.

\subsection{Simulation METHOD}

\subsubsection{DisCRETE PARTICLE SIMULATIONS}

When simulating non-spherical particles with discrete particle simulations, various different approaches can be taken [23], for example superquadrics [24-26], multi-spheres [27], polyhedra [28, 29] and spherocylinders [30]. Here, the implementation of ellipsoidal particles is done as a subset of superquadric particles, for which the implementation details in the open-source package MercuryDPM [31-34] can be found in appendix 5.A. Each particle has semi-axes $a, b$ and $c$; we look at spheres and prolate ellipsoids, where $a \geq b=c$. We define the aspect ratio $R=a / b$, where $R=1$ for spheres and $R>1$ for prolate ellipsoids. We use $a=\frac{R}{2 \sqrt[3]{R}}$ and $b=\frac{1}{2 \sqrt[3]{R}}$ to give all particles the same volume.

The system is non-dimensionalised such that each spherical particle has diameter $d=1$, mass $m=1$, and the magnitude of gravity is $g=1$. Since we are just interested in the influence of elongation of particles on segregation, the density of all particles is equal. We use a linear spring-dashpot contact-model [35], with a restitution coefficient $r_{c}=0.1$, contact-time $t_{c}=0.05$ and Coulomb friction coefficient $\mu=0.5$.

We simulate a rotating drum with bidisperse mixtures of spheres and ellipsoids with the same number of particles in each phase. We vary the aspect ratios of the ellipsoids for different mixtures, $R \in\{1,1.5,2,2.5,3,3.5,4\}$, where no segregation is expected for $R=1$ (sphere-sphere mixture). The rotating drum is constructed by fixing one layer of small particles $(d=0.5)$ to the bed. It has a diameter of $r=30$, and periodic boundary conditions in traverse direction. The full algorithm for rotating the drum is given in appendix 5.B. We set the rotation speed $\omega$ such that the ratio of centrifugal and gravitational forces, or Froude number, $F=\frac{\omega^{2} r}{g}$, equals 0.01 , which is around the upper limit of the rolling regime [5]. It has been verified by looking at snapshots that the flows are in the rolling regime and not the cascading regime. We look at the results after 10 rotations $(T \approx 2433)$, where He et al. [12] already saw strong qualitative differences for mixtures with $R=2$ and $R=4$ even though the mixture was not fully segregated yet. After these ten rotations, we restart the drum for half a rotation in order to do coarse graining. Repeating this process twice, we obtain three data sets that are taken from half a rotation each in the period 10 to 11.5 rotations. 


\subsubsection{ANALYSIS}

For the analysis of the discrete particle simulations, a coarse-graining method [20-22] is used to extract continuum fields that are averaged over both space and time. To compute the concentration of spheres $\phi$, coarse graining is applied for both the bulk of the flow and the different types of particles individually. This way, the concentration of spheres can be expressed as $\phi=\rho_{\text {sphere }} / \rho$, where $\rho_{\text {sphere }}$ is the (coarse grained) density of the sphere phase and $\rho$ the (coarse grained) density of the mixture as a whole. The coarse graining width $w=1$ is chosen based on a similar system by Hidalgo et al. [11], and Lucypolynomials [36] are chosen as coarse graining function for efficiency.

\section{SEgREgation INDEX}

Concerning the measure of segregation, several mixing indices exist, e.g. [2, 37-40]. Here, we adapt the method of $[2,40]$ that is based on mixing entropy of statistical mechanics: first, define the local mixing entropy using Boltzmann's expression

$$
m(\mathbf{r})=\phi(\mathbf{r}) \ln (\phi(\mathbf{r}))+(1-\phi(\mathbf{r})) \ln (1-\phi(\mathbf{r})),
$$

where it should be noted that the number fraction of spheres is the same as the density fraction $\phi$, because our particles all have equal density and equal volume. The global mixing entropy $\tilde{M}$ is then defined as the weighted integral of the local mixing entropy:

$$
\tilde{M}=\int_{\mathbb{R}^{3}} \rho(\mathbf{r}) m(\mathbf{r}) d \mathbf{r} .
$$

Finally, the global mixing index is normalised with the perfectly mixed state $\tilde{M}_{\text {mix }}$ and perfectly segregated state $\tilde{M}_{\text {seg, }}$,

$$
M=\frac{\tilde{M}-\tilde{M}_{\mathrm{seg}}}{\tilde{M}_{\mathrm{mix}}-\tilde{M}_{\mathrm{seg}}},
$$

so that $M=0$ corresponds to a perfectly segregated flow, and $M=1$ to a perfectly mixed flow. For a perfectly segregated flow, $\phi=0$ or $\phi=1$ at almost all points in space. Therefore, a lower bound for the mixing index is given by

$$
\begin{aligned}
\tilde{M}_{\text {seg }} & =\lim _{\phi \rightarrow 0} \int_{\mathbb{R}^{3}} \rho(\phi \ln (\phi)+(1-\phi) \ln (1-\phi)) d \mathbf{r} \\
& =0 .
\end{aligned}
$$

It should be noted that this lower bound is never attained, since even in the most segregated cases there is a division line between the phases around which the sphere-concentration is not exactly either 0 or 1 . The mixing index can come arbitrarily close to $M=0$ depending on drum size.

A perfectly mixed system, on the other hand, has a concentration of spheres $\phi=1 / 2$ at any point in space. For the mixing index, this implies that

$$
\begin{aligned}
\tilde{M}_{\text {mix }} & =\int_{\mathbb{R}^{3}} \rho(\mathbf{r})\left(\frac{1}{2} \ln \left(\frac{1}{2}\right)+\frac{1}{2} \ln \left(\frac{1}{2}\right)\right) d \mathbf{r}, \\
& =-\ln (2) \int_{\mathbb{R}^{3}} \rho(\mathbf{r}) d \mathbf{r} .
\end{aligned}
$$


Combining equations (5.2)-(5.7), the global mixing index can be expressed as

$$
M=-\frac{\int_{\mathbb{R}^{3}} \rho(\mathbf{r}) s(\mathbf{r}) d \mathbf{r}}{\ln (2) \int_{\mathbb{R}^{3}} \rho(\mathbf{r}) d \mathbf{r}} .
$$

As is shown in section 5.3 and [12], the spheres can segregate to either the core or the outside of the flow, and therefore we define a segregation index $S$ such that $S=0$ for a perfectly mixed flow, $S=1$ for a perfectly segregated flow with spheres at the periphery and $S=-1$ for a perfectly segregated flow with spheres at the core. Using the mixing index (5.8), the segregation index $S$ is thus defined as

$$
S= \begin{cases}M-1 & \text { if spheres segregate to core } \\ 1-M & \text { otherwise }\end{cases}
$$

To numerically determine the global segregation index, quadrature rules are used after evaluating the coarse grained fields at discrete points.

\subsection{RESULTS}

\subsubsection{SEGREGATION AND INVERSE SEGREGATION}

To study the influence of particle elongation on segregation, mixtures of equal parts spheres and prolate ellipsoids are simulated in a rotating drum as described in section 5.2.1. Figures 5.2 and 5.3 show a snapshot of the particles at 10 rotations and the coarse grained sphere concentration averaged over the time-period of $10-11.5$ rotations.

For all mixtures of spheres and prolate ellipsoids with $R>1$, radial segregation is observed. For mixtures where the aspect-ratio is relatively low, $1.5 \leq R \leq 3$, the ellipsoids segregate towards the core of the flow, where ellipsoids with large aspect ratio $R \geq 3.5$ segregate towards the outside. This is consistent with He et al. [12], who looked only at the aspect ratios $R=2$ and $R=4$.

We further observe that all simulations have one layer at the periphery that contains a higher concentration of spheres, even in the $R \geq 3.5$ cases. This is due to two reasons: firstly, many ellipsoids are oriented with their long axis orthogonal to the drum wall, thus the center of mass of ellipsoidal particles touching the wall is further from the wall than for spherical particles touching the wall. More interestingly, there is a higher spheredensity along the periphery (figure not shown). Looking at the snapshots and figure 2(b) of [12], those simulations for $R=4$ also seem to have the higher density of spheres along the periphery, although this cannot be said for sure without coarse graining the data. Since their drum is much larger, has a smooth surface and end-walls, this indicates that it is likely not an artifact of the drum construction, but rather an unknown percolation effect.

\subsubsection{SEGREGATION INDEX}

To quantify the segregation direction and strength, we measure the mixing index S from the coarse grained concentration profiles: for $R=1$, there is no significant segregation, the segregation strength rapidly increases until $R=2$ and then decreases more slowly until the direction flips between $R=3$ and $R=3.5$. After the direction of segregation 

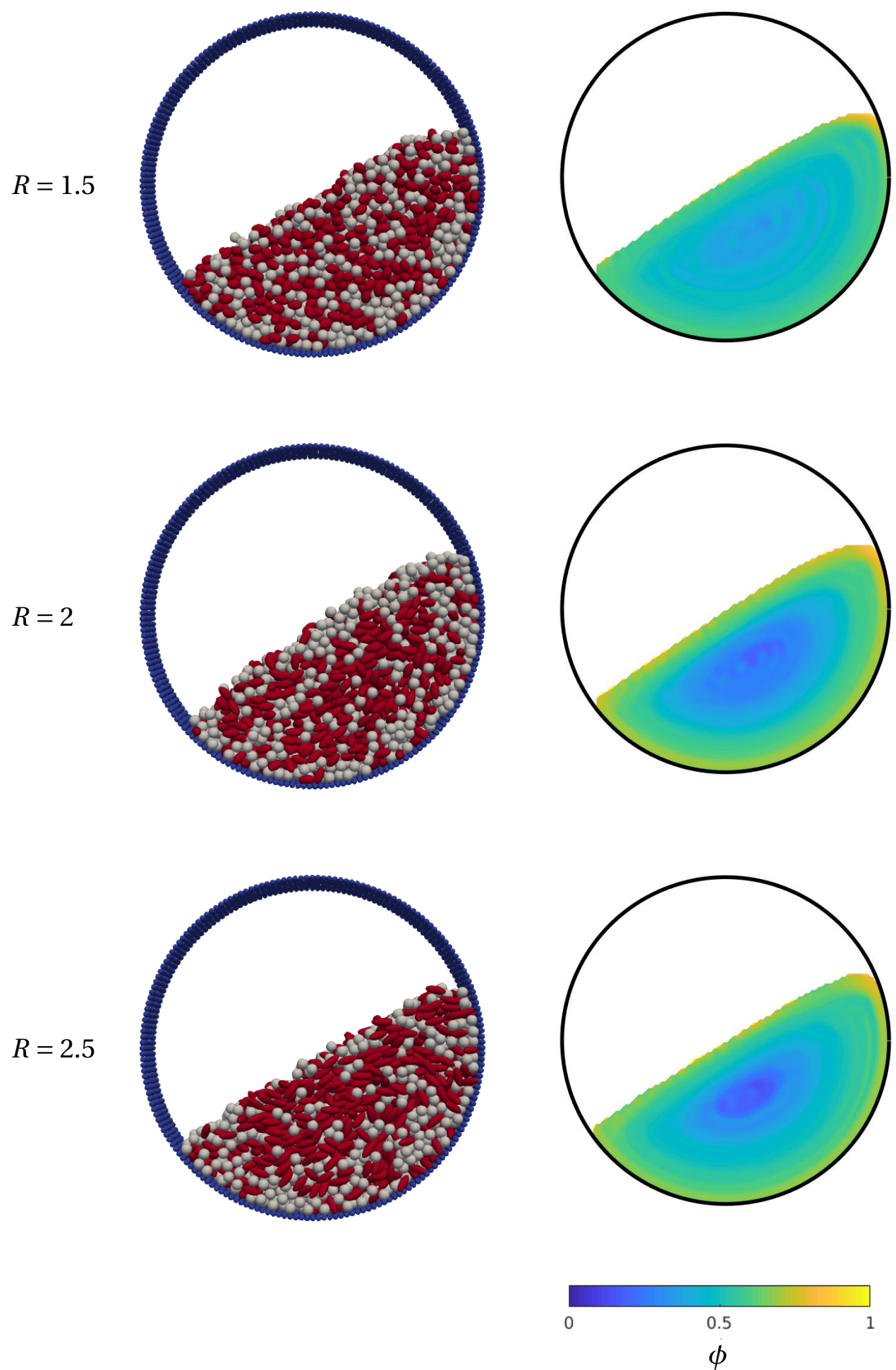

Figure 5.2: Snapshots and coarse grained sphere-concentration of mixtures of spheres and ellipsoids with aspect-ratios $R=1.5$ (top), $R=2$ (middle) and $R=2.5$ (bottom) 

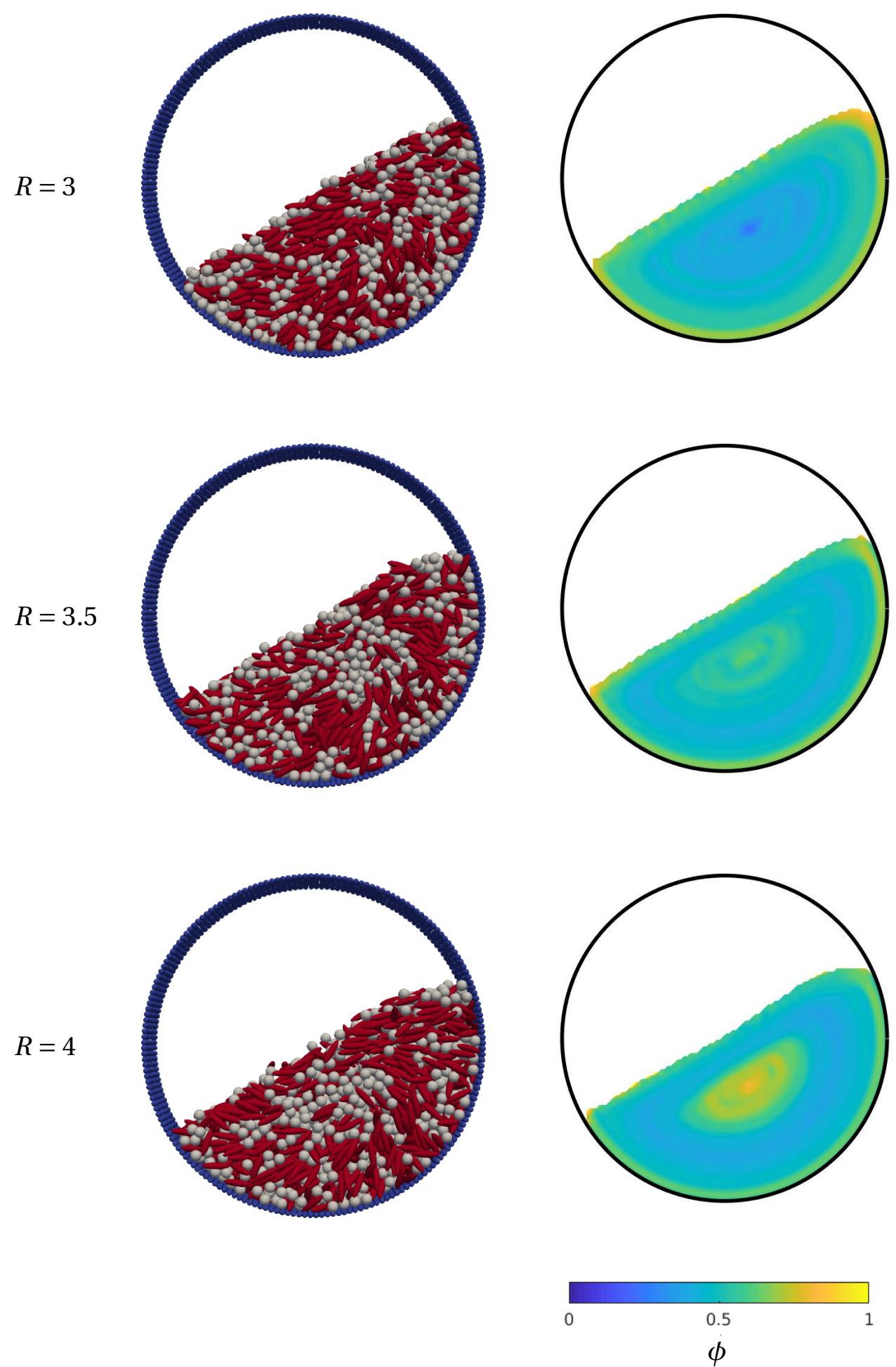

Figure 5.3: Snapshots and coarse grained sphere-concentration of mixtures of spheres and ellipsoids with aspect-ratios $R=3$ (top), $R=3.5$ (middle) and $R=4$ (bottom) 


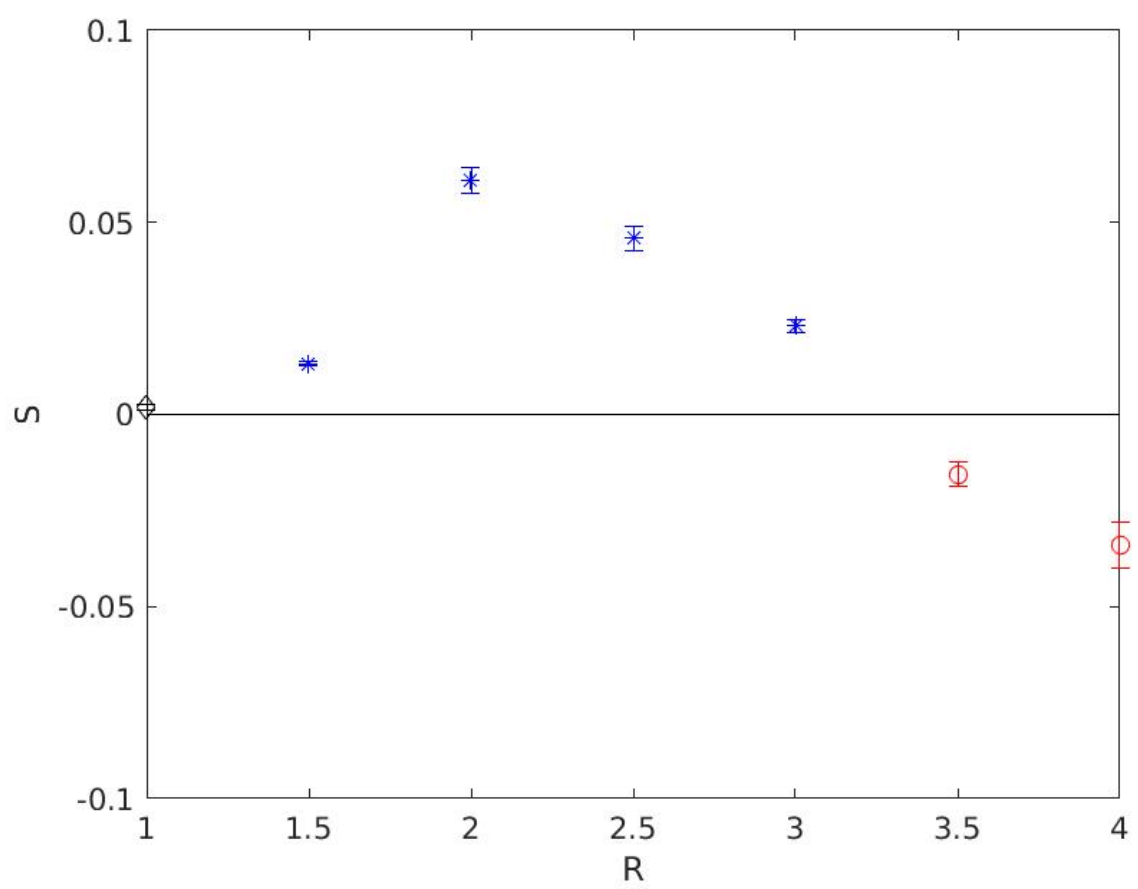

Figure 5.4: Segregation index for mixtures of spheres and ellipsoids of various aspect ratios. Black diamond: no segregation, blue stars: ellipsoids segregate to the core, red circles: ellipsoids segregate to the periphery. Errorbars denote the standard deviation.

flipped, the strength of segregation increases again. This data supports the hypothesis of [12] that there are two competing mechanisms at play, namely a difference of flowability, which causes the ellipsoids to segregate to the centre, and a percolation effect for mixtures with ellipsoids with high aspect ratio, causing the spheres to segregate to the centre.

\subsection{CONCLUSION AND DISCUSSION}

In this chapter, we have shown that modeling segregation based on particle elongation is not straight-forward. Using mixtures of spheres and prolate ellipsoids of various aspect ratios, we observe that for mixtures with ellipsoids with small aspect ratios $(R \leq 3)$, the ellipsoids segregate to the core of the flow, and the spheres to the periphery. For mixtures with ellipsoids with large aspect ratios $(R \geq 3.5)$, we see that the direction of segregation reverses, and spheres segregate toward the core and the ellipsoids to the periphery.

The strength of segregation is also not monotone: in a mixture between spheres and ellipsoids with $R=1$ (spheres), the flow of course does not segregate. Somewhere in the interval $R \in(1.5,2.5)$, the maximum segregation strength is attained, after which the segregation strength decreases again until the segregation direction reverses and the 
strength increases again in that direction. This indicates that there are at least two segregation mechanisms at play, which is also hypothesised by [12], who only used two different aspect ratios $(R=2$ and $R=4)$.

The first hypothesised effect is a difference in flowability: spheres have a higher flowability, and therefore segregate to the outer periphery $[12,13]$. This hypothesis can be tested in the future by looking at both the surface speed and the energy dissipation associated with each species in the flowing layer. If the ellipsoidal particles have a lower flowability, it is likely that they come to rest higher up along the surface than the spheres, and hence segregate to the core.

The second hypothesised effect is percolation of some form. This is much harder to test, but information on the location of segregation might help here. If the segregation takes place in the sheared layer, then the behaviour of segregation should be similar to that in periodic chute flows, which are studied in depth in other work [11, 14]. If, on the other hand, segregation takes place in the solid body regime, there is almost surely a percolation effect at play that cannot be captured by the simple chute flows. In order to study the location of the segregation, one can look at the velocity fields of each type of particle and compare it to the velocity field of the bulk of the flow. This needs to be averaged over many time steps in the time frame when the flow is segregating, to filter out the random fluctuations of the velocity fields.

Another possible effect is the minimisation of void spaces, or potential energy, as is seen in vibrated granular systems [41, 42]. Especially for ellipsoids with larger aspectratios, this means that they clump together and line up. We do seem to see a preferred orientation of the ellipsoids with large aspect-ratios, but this may be solely due to them having a preferred orientation in the sheared region [7], combined with a solid body rotation. It would be interesting to see both the angle of the ellipsoids compared to the flow direction, and the difference in void volume throughout the flow.

Other than seeking the cause of segregation of particles with different aspect ratios, there is still much to be learned phenomenologically: what happens with the segregation index both before the maximum is attained $(R \in(1,2.5))$ and when there is almost no segregation $(R \in(3,3.5))$ ? In order to construct a continuum model for segregation based on particle elongation, the time-scale on which segregation occurs is also essential, as is the influence of system parameters like rotation speed and drum size. Lastly, it should be studied if segregation based on elongation behaves similarly in different geometries, such as chute flows and heap flows, or if it is unique to the system of rotating drums.

\section{REFERENCES}

[1] J. M. Ottino and D. V. Khakhar, Mixing and segregation of granular materials, Annual review of fluid mechanics 32, 55 (2000).

[2] M. M. H. D. Arntz, H. H. Beeftink, W. K. den Otter, W. J. Briels, and R. M. Boom, Segregation of granular particles by mass, radius, and density in a horizontal rotating drum, AIChE journal 60, 50 (2014).

[3] J. M. N. T. Gray, Particle segregation in dense granular flows, Annual Review of Fluid Mechanics 50, 407 (2018). 
[4] H. Henein, J. K. Brimacombe, and A. P. Watkinson, Experimental study of transverse bed motion in rotary kilns, Metallurgical transactions B 14, 191 (1983).

[5] J. Mellmann, The transverse motion of solids in rotating cylinders-forms of motion and transition behavior, Powder technology 118, 251 (2001).

[6] I. Govender, Granular flows in rotating drums: A rheological perspective, Minerals Engineering 92, 168 (2016).

[7] H. Ma and Y. Zhao, Modelling of the flow of ellipsoidal particles in a horizontal rotating drum based on dem simulation, Chemical Engineering Science 172, 636 (2017).

[8] K. A. Reddy, V. Kumaran, and J. Talbot, Orientational ordering in sheared inelastic dumbbells, Physical Review E 80, 031304 (2009).

[9] C. S. Campbell, Elastic granular flows of ellipsoidal particles, Physics of Fluids 23, 013306 (2011).

[10] T. Börzsönyi, B. Szabó, S. Wegner, K. Harth, J. Török, E. Somfai, T. Bien, and R. Stannarius, Shear-induced alignment and dynamics of elongated granular particles, Physical Review E 86, 051304 (2012).

[11] R. Hidalgo, B. Szabó, K. Gillemot, T. Börzsönyi, and T. Weinhart, Rheological response of nonspherical granular flows down an incline, Physical Review Fluids $\mathbf{3}$, 074301 (2018).

[12] S. Y. He, J. Q. Gan, D. Pinson, and Z. Y. Zhou, Particle shape-induced radial segregation of binary mixtures in a rotating drum, Powder Technology (2018).

[13] G. Pereira and P. Cleary, Segregation due to particle shape of a granular mixture in a slowly rotating tumbler, Granular Matter 19, 23 (2017).

[14] S. Mandal and D. V. Khakhar, Dense granular flow of mixtures of spheres and dumbbells down a rough inclined plane: Segregation and rheology, Physics of Fluids 31, 023304 (2019).

[15] GDR-MiDi, On dense granular flows, The European Physical Journal E 14, 341 (2004).

[16] P. Jop, Y. Forterre, and O. Pouliquen, A constitutive law for dense granular flows, Nature 441, 727 (2006).

[17] J. M. N. T. . Gray and V. A. Chugunov, Particle-size segregation and diffusive remixing in shallow granular avalanches, Journal of Fluid Mechanics 569, 365 (2006).

[18] Y. Zhao, H. Xiao, P. B. Umbanhowar, and R. M. Lueptow, Simulation and modeling of segregating rods in quasi-2d bounded heap flow, AIChE Journal 64, 1550 (2018).

[19] M. Alizadeh, A. Hassanpour, M. Pasha, M. Ghadiri, and A. Bayly, The effect of particle shape on predicted segregation in binary powder mixtures, Powder technology 319, 313 (2017). 
[20] I. Goldhirsch, Stress, stress asymmetry and couple stress: from discrete particles to continuous fields, Granular Matter 12, 239 (2010).

[21] T. Weinhart, A. R. Thornton, S. Luding, and O. Bokhove, Closure relations for shallow granular flows from particle simulations, Granular Matter 14, 531 (2012).

[22] D. R. Tunuguntla, A. R. Thornton, and T. Weinhart, From discrete elements to continuum fields: Extension to bidisperse systems, Computational particle mechanics 3, 349 (2016).

[23] G. Lu, J. R. Third, and C. R. Müller, Discrete element models for non-spherical particle systems: from theoretical developments to applications, Chemical Engineering Science 127, 425 (2015).

[24] A. H. Barr, Superquadrics and angle-preserving transformations, IEEE Computer graphics and Applications 1, 11 (1981).

[25] P. W. Cleary, Large scale industrial dem modelling, Engineering Computations 21, 169 (2004).

[26] A. Podlozhnyuk, S. Pirker, and C. Kloss, Efficient implementation of superquadric particles in discrete element method within an open-source framework, Computational Particle Mechanics 4, 101 (2017).

[27] H. Kruggel-Emden, S. Rickelt, S. Wirtz, and V. Scherer, A study on the validity of the multi-sphere discrete element method, Powder Technology 188, 153 (2008).

[28] P. A. Cundall, Formulation of a three-dimensional distinct element model-part i. a scheme to detect and represent contacts in a system composed of many polyhedral blocks, in International Journal of Rock Mechanics and Mining Sciences \& Geomechanics Abstracts, Vol. 25 (Elsevier, 1988) pp. 107-116.

[29] R. Hart, P. A. Cundall, and J. Lemos, Formulation of a three-dimensional distinct element model_part ii. mechanical calculations for motion and interaction of a system composed of many polyhedral blocks, in International Journal of Rock Mechanics and Mining Sciences \& Geomechanics Abstracts, Vol. 25 (Elsevier, 1988) pp. 117-125.

[30] S. M. Rubio-Largo, F. Alonso-Marroquin, T. Weinhart, S. Luding, and R. Hidalgo, Homogeneous cooling state of frictionless rod particles, Physica A: Statistical Mechanics and its Applications 443, 477 (2016).

[31] MercuryDPM, https : //www . mercurdpm . org, last accessed on August 27th 2019.

[32] A. R. Thornton, T. Weinhart, S. Luding, and O. Bokhove, Modeling of particle size segregation: calibration using the discrete particle method, International Journal of Modern Physics C 23, 1240014 (2012).

[33] T. Weinhart, A. R. Thornton, S. Luding, and O. Bokhove, From discrete particles to continuum fields near a boundary, Granular Matter 14, 289 (2012). 
[34] T. Weinhart, M. Post, I. F. C. Denissen, D. R. Tunuguntla, E. Grannonio, N. Losacco, J. Barbosa, W. K. Den Otter, and A. R. Thornton, Fast, flexible particle simulations: An introduction to MercuryDPM, in Proceedings of the 8th International Conference on Discrete Element Methods (2019).

[35] S. Luding, Introduction to discrete element methods: basic of contact force models and how to perform the micro-macro transition to continuum theory, European Journal of Environmental and Civil Engineering 12, 785 (2008).

[36] L. B. Lucy, A numerical approach to the testing of the fission hypothesis, The astronomical journal 82, 1013 (1977).

[37] P. W. Cleary, G. Metcalfe, and K. Liffman, How well do discrete element granular flow models capture the essentials of mixing processes? Applied Mathematical Modelling 22, 995 (1998).

[38] C. M. Dury and G. H. Ristow, Competition of mixing and segregation in rotating cylinders, Physics of Fluids 11, 1387 (1999).

[39] P. Porion, N. Sommier, A. Faugere, and P. Evesque, Dynamics of size segregation and mixing of granular materials in a $3 d$-blender by nmr imaging investigation, Powder Technology 141, 55 (2004).

[40] M. M. H. D. Arntz, W. K. den Otter, W. J. Briels, P. J. T. Bussmann, H. H. Beeftink, and R. M. Boom, Granular mixing and segregation in a horizontal rotating drum: $a$ simulation study on the impact of rotational speed and fill level, AIChE journal 54, 3133 (2008).

[41] C. R. A. Abreu, F. W. Tavares, and M. Castier, Influence of particle shape on the packing and on the segregation of spherocylinders via monte carlo simulations, Powder Technology 134, 167 (2003).

[42] C. R. K. Windows-Yule, B. J. Scheper, W. K. den Otter, D. J. Parker, and A. R. Thornton, Modifying self-assembly and species separation in three-dimensional systems of shape-anisotropic particles, Physical Review E 93, 020901 (2016). 


\section{APPENDiX}

\section{A. IMPLEMENTATION OF SUPERQUADRIC PARTICLES IN MER- CURYDPM}

This appendix gives an overview and some of the details for how superquadric particles are implemented in MercuryDPM [1-4]. The description of the geometry is based on chapter 2 of Jaklic et al. [5], while the contact-detection algorithm is heavily based on the work of Podlozhnyuk et al. [6]. Usually, only the results that are relevant for implementation in MercuryDPM are given; for derivations of the formulas we advise the interested reader to look at these two resources. First, we describe the representation of the particle, some of its intrinsic properties and its implementation in MercuryDPM. Then we will look at the contact-detection algorithm and contact-force computation. This appendix finishes with an outlook on other features that should be implemented.

\section{A.1. Particle Properties}

\section{SHAPE REPRESENTATION}

There are multiple possible representations of superquadric particles, where one can represent a particle by either a continuous shape function (continuous function representation), or an array of discrete points (discrete function representation) [7]. Here, we use a continuous function representation, using the same formulation as Barr [8] and Jaklic et al. [5] to describe the surface of a particle via the shape function

$$
f(\mathbf{x})=\left(\left|\frac{x}{a}\right|^{2 / \epsilon_{2}}+\left|\frac{y}{b}\right|^{2 / \epsilon_{2}}\right)^{\epsilon_{2} / \epsilon_{1}}+\left|\frac{z}{c}\right|^{2 / \epsilon_{1}}-1 .
$$

Here, $a, b$ and $c$ are the lengths of the semi-axes of the superquadric particle in $x, y$ and $z$ direction respectively. $\epsilon_{1}$ and $\epsilon_{2}$ are the blockiness parameters; we constrain ourselves to the strictly convex shapes with $0<\epsilon_{1} \leq 1$ and $0<\epsilon_{2} \leq 1$. Choosing $\epsilon_{1}=\epsilon_{2}=1$ corresponds to ellipsoid shapes, $\epsilon_{1} \ll 1, \epsilon_{2}=1$ corresponds to cylinders, $\epsilon_{1}=1, \epsilon_{2} \ll 1$ corresponds to pillows and $\epsilon_{1} \ll 1, \epsilon_{2} \ll 1$ corresponds to blocks, see figure 5.A.1 for some examples of superquadric particles.

The surface of the superquadric is defined as all the points where $f(\mathbf{x})=0$. For negative values of $f(\mathbf{x}), \mathbf{x}$ is in the interior of the particle, while $f(\mathbf{x})$ is positive for $\mathbf{x}$ outside the particle. Note, that this is all in a body-fixed coordinate system $\mathbf{x}=[x, y, z]^{T}$, which is the coordinate system where the centre of the particle is at the origin and the particle is not rotated.

To compute the gradient of the shape function (5.10), and thus the normal direction of contact, we follow [6], and define

$$
v=\left|\frac{x}{a}\right|^{2 / \epsilon_{2}}+\left|\frac{y}{b}\right|^{2 / \epsilon_{2}} .
$$




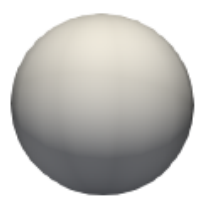

$$
a=b=c
$$$$
\epsilon_{1}=1, \epsilon_{2}=1
$$

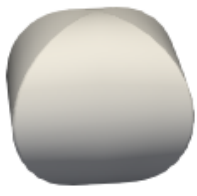

$$
a=b=c
$$

$$
\epsilon_{1}=1, \epsilon_{2} \ll 1
$$

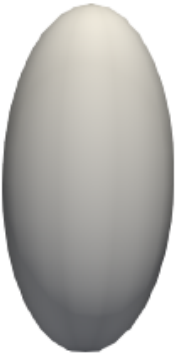

$a=b<c$

$\epsilon_{1}=1, \epsilon_{2}=1$

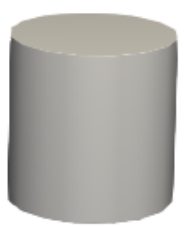

$a=b=c$

$\epsilon_{1} \ll 1, \epsilon_{2}=1$

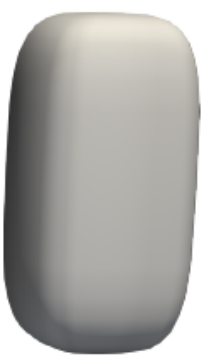

$a=b<c$

$\epsilon_{1}=0.5, \epsilon_{2}=0.5$

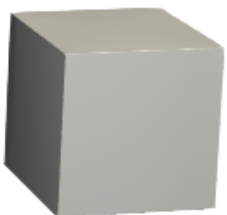

$a=b=c$

$\epsilon_{1} \ll 1, \epsilon_{2} \ll 1$

Figure 5.A.1: A variety of shapes that can be represented by (strictly) convex superquadric particles. Note, that even though the edges for the shapes on the bottom rows seem sharp and their faces flat, they are actually rounded such that they are still strictly convex and therefore can have only one contact-point with another particle or non-concave wall. 
With this definition, the gradient of the shape function can be written as

$$
\nabla_{\mathbf{x}} f(\mathbf{x})=\left(\begin{array}{c}
\frac{2}{\epsilon_{1} a}\left|\frac{x}{a}\right|^{2 / \epsilon_{2}-1} v^{\epsilon_{2} / \epsilon_{1}-1} \operatorname{sign}(x) \\
\frac{2}{\epsilon_{1} b}\left|\frac{y}{b}\right|^{2 / \epsilon_{2}-1} v^{\epsilon_{2} / \epsilon_{1}-1} \operatorname{sign}(y) \\
\frac{2}{\epsilon_{1} c}\left|\frac{z}{c}\right|^{2 / \epsilon_{1}-1} \operatorname{sign}(z)
\end{array}\right) .
$$

In the special case of a sphere or ellipsoid, $\epsilon_{1}=\epsilon_{2}=1$, this expression is much simpler:

$$
\nabla_{\mathbf{x}} f_{\text {ellipsoid }}(\mathbf{x})=\left(\begin{array}{c}
\frac{2 x}{a^{2}} \\
\frac{2 y}{b^{2}} \\
\frac{2 z}{c^{2}}
\end{array}\right)
$$

Furthermore, the contact-detection algorithm in section 5.A.2 also needs the Hessian of the shape function of the particle. The Hessian of shape function (5.10) is given by

$$
\mathbf{H}_{\mathbf{x}}(f)(\mathbf{x})=\left(\begin{array}{ccc}
H_{\mathbf{x}}^{11} & H_{\mathbf{x}}^{12} & 0 \\
H_{\mathbf{x}}^{12} & H_{\mathbf{x}}^{22} & 0 \\
0 & 0 & H_{\mathbf{x}}^{33}
\end{array}\right)
$$

where

$$
\begin{aligned}
& H_{\mathbf{x}}^{11}(\mathbf{x})=\frac{2}{\epsilon_{1} a^{2}}\left(\frac{2}{\epsilon_{2}}-1\right)\left|\frac{x}{a}\right|^{2 / \epsilon_{2}-2} v^{\epsilon_{2} / \epsilon_{1}-1}+\frac{2}{\epsilon_{1} a^{2}}\left(\frac{2}{\epsilon_{1}}-\frac{2}{\epsilon_{2}}\right)\left|\frac{x}{a}\right|^{4 / \epsilon_{2}-2} v^{\epsilon_{2} / \epsilon_{1}-2}, \\
& H_{\mathbf{x}}^{22}(\mathbf{x})=\frac{2}{\epsilon_{1} b^{2}}\left(\frac{2}{\epsilon_{2}}-1\right)\left|\frac{y}{b}\right|^{2 / \epsilon_{2}-2} v^{\epsilon_{2} / \epsilon_{1}-1}+\frac{2}{\epsilon_{1} b^{2}}\left(\frac{2}{\epsilon_{1}}-\frac{2}{\epsilon_{2}}\right)\left|\frac{y}{b}\right|^{4 / \epsilon_{2}-2} v^{\epsilon_{2} / \epsilon_{1}-2}, \\
& H_{\mathbf{x}}^{33}(\mathbf{x})=\frac{2}{\epsilon_{1} c^{2}}\left(\frac{2}{\epsilon_{1}}-1\right)\left|\frac{z}{c}\right|^{2 / \epsilon_{1}-2}, \\
& H_{\mathbf{x}}^{12}(\mathbf{x})=\frac{2}{\epsilon_{1} a b}\left(\frac{2}{\epsilon_{1}}-\frac{2}{\epsilon_{2}}\right)\left|\frac{x}{a}\right|^{2 / \epsilon_{2}-1}\left|\frac{y}{b}\right|^{2 / \epsilon_{2}-1} v^{\epsilon_{2} / \epsilon_{1}-2} \operatorname{sign}(x y) .
\end{aligned}
$$

Again, for an ellipsoid $\left(\epsilon_{1}=\epsilon_{2}=1\right)$ this can be simplified:

$$
\mathbf{H}_{\mathbf{x}}\left(f_{\text {ellipsoid }}\right)(\mathbf{x})=\left(\begin{array}{ccc}
2 / a^{2} & 0 & 0 \\
0 & 2 / b^{2} & 0 \\
0 & 0 & 2 / c^{2}
\end{array}\right) \text {. }
$$

\section{LAB-FIXED AND BODY-FIXED COORDINATE SYSTEMS}

In contrast with spheres, superquadric particles have an anisotropic geometry. This means that for each particle, it is important to know its orientation. In MercuryDPM, the rotation of an object is represented by a quaternion

$$
\mathbf{q}=\left[\begin{array}{llll}
q_{0} & q_{1} & q_{2} & q_{3}
\end{array}\right]^{T} .
$$

Using quaternions, the rotation matrix $A$ of a particle can be defined as [6]

$$
A=\left(\begin{array}{ccc}
1-2\left(q_{2}^{2}+q_{3}^{2}\right) & 2\left(q_{1} q_{2}-q_{0} q_{3}\right) & 2\left(q_{1} q_{3}+q_{0} q_{2}\right) \\
2\left(q_{1} q_{2}+q_{0} q_{3}\right) & 1-2\left(q_{1}^{2}+q_{3}^{2}\right) & 2\left(q_{2} q_{3}-q_{0} q_{1}\right) \\
2\left(q_{1} q_{3}-q_{0} q_{2}\right) & 2\left(q_{2} q_{3}+q_{0} q_{1}\right) & 1-2\left(q_{1}^{2}+q_{2}^{2}\right)
\end{array}\right) .
$$


This matrix is orthogonal, i.e. $A^{-1}=A^{T}$. In MercuryDPM, the rotation matrix $A$ can be obtained with the method Quaternion: : getA. Alternatively, a vector can be multiplied with $A$ (and thus rotated) via the method Quaternion: : rotate, and multiplied with $A^{T}$ via Quaternion: : rotateBack.

One can now find the body-fixed position $\mathbf{x}$ from the lab-fixed position $\mathbf{X}$ and centre of particle $i, \mathbf{X}_{i}$, as

$$
\mathbf{X}=A^{T}\left(\mathbf{X}-\mathbf{X}_{i}\right)
$$

The shape function $F$ in terms of the lab-fixed coordinates $\mathbf{X}$ then equals

$$
F(\mathbf{X})=f(\mathbf{x})
$$

and the gradient and Hessian of this lab-fixed shape function are given by

$$
\begin{aligned}
\nabla_{\mathbf{X}} F(\mathbf{X}) & =A \nabla_{\mathbf{X}} f(\mathbf{x}), \\
\mathbf{H}_{\mathbf{X}}(F)(\mathbf{X}) & =A \mathbf{H}_{\mathbf{X}}(f)(\mathbf{x}) A^{T} .
\end{aligned}
$$

\section{VOLUME AND INERTIA TENSOR}

In order to resolve the behaviour of the particles correctly, we need the volume and inertia tensor of each particle. The equations for the volume $V$ and inertia-tensor $I$ are derived in chapter 2 of [5], assuming a homogeneous particle density inside the particle, and are repeated here for completeness.

$$
\begin{aligned}
V & =2 a b c \epsilon_{1} \epsilon_{2} B\left(\frac{\epsilon_{1}}{2}+1, \epsilon_{1}\right) B\left(\frac{\epsilon_{2}}{2}, \frac{\epsilon_{2}}{2}\right), \\
I & =\frac{\rho}{2} a b c \epsilon_{1} \epsilon_{2}\left(\begin{array}{ccc}
I_{x x} & 0 & 0 \\
0 & I_{y y} & 0 \\
0 & 0 & I_{z z}
\end{array}\right), \\
I_{x x} & =\left(b^{2} B\left(\frac{3}{2} \epsilon_{2}, \frac{1}{2} \epsilon_{2}\right) B\left(\frac{1}{2} \epsilon_{1}, 2 \epsilon_{1}+1\right)+4 c^{2} B\left(\frac{1}{2} \epsilon_{2}, \frac{1}{2} \epsilon_{2}+1\right) B\left(\frac{3}{2} \epsilon_{1}, \epsilon_{1}+1\right)\right), \\
I_{y y} & =\left(a^{2} B\left(\frac{3}{2} \epsilon_{2}, \frac{1}{2} \epsilon_{2}\right) B\left(\frac{1}{2} \epsilon_{1}, 2 \epsilon_{1}+1\right)+4 c^{2} B\left(\frac{1}{2} \epsilon_{2}, \frac{1}{2} \epsilon_{2}+1\right) B\left(\frac{3}{2} \epsilon_{1}, \epsilon_{1}+1\right)\right), \\
I_{z z} & =\left(a^{2}+b^{2}\right) B\left(\frac{3}{2} \epsilon_{2}, \frac{1}{2} \epsilon_{2}\right) B\left(\frac{1}{2} \epsilon_{1}, 2 \epsilon_{1}+1\right),
\end{aligned}
$$

where $\rho$ is the density of a particle and $B(x, y)$ is the beta function

$$
B(x, y)=2 \int_{0}^{\pi / 2} \sin ^{2 x-1} \phi \cos ^{2 y-1} \phi d \phi .
$$

Note that the inertia-tensor in the body-fixed coordinate system is an intrinsic property of the particle and does not have to be recomputed every time-step. The inertia tensor for the lab-fixed coordinate system can then be computed as $I_{\mathbf{X}}=A I A^{T}$.

\section{BOUNDING SPHERE}

Another important intrinsic property of a particle is the radius of its minimal bounding sphere, namely the maximum distance from the centre of the particle to its surface. 
In the body-fixed coordinate system, this can be formulated as:

$$
\begin{aligned}
& \text { maximise }\left(r_{\text {bounding }}\right)^{2} \equiv x^{2}+y^{2}+z^{2}, \\
& \text { subject to } f(\mathbf{x})=0 .
\end{aligned}
$$

This optimisation problem has been solved by [6], with result

$$
\begin{aligned}
r_{\text {bounding }} & =\tilde{x} \sqrt{a^{2}+\alpha^{2} b^{2}+\beta^{2} c^{2}}, \text { where } \\
\tilde{x} & =\left(\left(1+\alpha^{2 / \epsilon_{2}}\right)^{\epsilon_{2} / \epsilon_{1}}+\beta^{2 / \epsilon_{1}}\right)^{-\epsilon_{1} / 2}, \\
\alpha & =(b / a)^{\epsilon_{2} /\left(1-\epsilon_{2}\right)}, \\
\gamma & =\left(1+\alpha^{2 / \epsilon_{2}}\right)^{\epsilon_{2} / \epsilon_{1}-1}, \\
\beta & =\left(\gamma c^{2} / a^{2}\right)^{\epsilon_{1} /\left(2-2 \epsilon_{1}\right)} .
\end{aligned}
$$

Note, that if $\epsilon_{1}=1$ or $\epsilon_{2}=1, \beta$ or $\alpha$ has an exponent of the form $1 / 0$. We therefore put in special cases for $\epsilon_{1}=1$ or $\epsilon_{2}=1$. When $\epsilon_{1}=\epsilon_{2}=1$ (ellipsoids), the radius of the minimal bounding sphere is simply the maximum length of the half-axes, $r_{\text {bounding }}=\max (a, b, c)$.

In the case $\epsilon_{2}=1$ and $\epsilon_{1} \ll 1$, we recognise that the shape function (5.10) can be rewritten as

$$
f(\mathbf{x})=\left(\left(\left(\frac{x}{a}\right)^{2}+\left(\frac{y}{b}\right)^{2}\right)^{1 / 2}\right)^{2 / \epsilon_{1}}+\left|\frac{z}{c}\right|^{2 / \epsilon_{1}}-1,
$$

which means that the shape is always an ellipse when projected on the $x y$-plane. Therefore, define $\tilde{a}=\max (a, b)$. Analogously to (5.25)-(5.29), we get the radius of the minimal bounding sphere

$$
\begin{aligned}
r_{\text {bounding }} & =\tilde{x} \sqrt{\tilde{a}^{2}+(\beta c)^{2}}, \text { where } \\
\tilde{x} & =\left(1+\beta^{2 / \epsilon_{1}}\right)^{-\epsilon_{1} / 2}, \\
\beta & =\left(c^{2} / \tilde{a}^{2}\right)^{\epsilon_{1} /\left(2-2 \epsilon_{1}\right)} .
\end{aligned}
$$

For the last case, $\epsilon_{1}=1$ and $\epsilon_{2} \ll 1$, we notice that the exponent in (5.29) is infinite. If $a \geq \sqrt{\gamma} c$, one can use (5.25) with $\beta=0$. On the other hand, if $a<\sqrt{\gamma} c$, then (5.25) - (5.29) would give a minimal bounding sphere with infinite radius, which is of course non-physical. The computation of the minimal bounding sphere for these particles should be worked out in the future, but for now it is sufficient to have a bounding sphere that is somewhat larger than the minimal bounding sphere for this case. To that end, we realise that for any $0<\epsilon_{1}, \epsilon_{2} \leq 1$, the sphere with radius $r_{\text {bounding }}=\sqrt{a^{2}+b^{2}+c^{2}}$ is a bounding sphere, although it is not the minimal bounding sphere. The only drawback of using a bigger bounding sphere than strictly necessary is that the method will result in more possible contacts, and therefore more computation time. The accuracy of the contact-detection and force-computation are not affected by this temporary solution.

\section{IMPLEMENTATION IN MERCURYDPM}

For the implementation in MercuryDPM, we decided to consistently use lab-fixed coordinates in the signatures of relevant methods/functions, as to avoid confusion. The 
shape-function and its gradient and Hessian are computed in the class Superquadric, which derives from BaseParticle. The value of the shape function $F(\mathbf{X})$ is computed in SuperQuadric: : computeShape, which takes a lab-fixed position. The gradient $\nabla_{\mathbf{X}} F(\mathbf{X})$ can be obtained with the method SuperQuadric: : computeShapeGradientLabFixed, which takes a lab-fixed position and returns the gradient of the shape with respect to the lab-fixed coordinates, see equation (5.20). The Hessian $H_{\mathbf{X}}(F)$ can be obtained with the method SuperQuadric: : computeHessianLabFixed, which takes a lab-fixed position and returns the Hessian (matrix) with respect to the lab-fixed coordinates, see equation (5.21). The computation of the shape-function and its gradient and Hessian are tested in the unit test ShapeGradientHessianUnitTest in the folder SuperQuadricDemos/UnitTests.

The computation of the volume $V$ and body-fixed inertia-tensor $I$ of a particle are implemented in SuperQuadric: :getVolume() and SuperQuadric: :setInertia() respectively, and tested for ellipsoids in VolumeUnitTest and InertiaTensorUnitTest in the folder SuperQuadricDemos/UnitTests. The computation of the radius of the minimal bounding sphere for a particle is done in SuperQuadric: : get InteractionRadius, which overwrites BaseParticle: : getInteractionRadius. It is tested in the unit test BoundingRadiusUnitTest in the folder SuperQuadricDemos/UnitTests.

\section{A.2. ContaCt DETECTION}

Knowing the properties of a particle, the next step is to implement the contact-detection algorithm. We use a three-stage contact detection algorithm: first, we check if the particle centres are sufficiently close together with the help of the hierarchical grid (HGrid) [9], where the HGrid-level of a particle is determined by the radius of its bounding sphere, as defined in section 5.A.1. If the distance between two particles is sufficiently small in the HGrid, we look at the bounding spheres of the particles. If the bounding spheres of these two particles overlap, the last stage looks at the detailed shape function of the particles. Similarly, for particle-wall contact detection we look first for the overlap between the bounding sphere with a wall, before looking at the detailed shape function of the particle. For details about the neighbourhood detection in the HGrid, we refer the interested reader to $[9,10]$. In this work, we only look at the last stage of contact-detection.

\section{Particle-Particle Contact Detection}

Note: in this section, all coordinates are in the lab-fixed coordinate system.

Assume we have two particles $i$ and $j$, and we want to know if those particles are in contact. Since both particles are strictly convex, this can be formulated as an optimisation problem as follows:

$$
\begin{gathered}
\operatorname{minimise} F_{i}\left(\mathbf{X}_{c}\right)+F_{j}\left(\mathbf{X}_{c}\right), \\
\text { subject to } F_{i}\left(\mathbf{X}_{c}\right)=F_{j}\left(\mathbf{X}_{c}\right) .
\end{gathered}
$$

Particles $i$ and $j$ are in contact if and only if $F_{i}\left(\mathbf{X}_{c}\right)=F_{j}\left(\mathbf{X}_{c}\right)<0$ for the resulting $\mathbf{X}_{c}$. We then define $\mathbf{X}_{c}$ as the contact point.

We follow [6] and use a Lagrange-multiplier method to solve the system (5.34)-(5.35). Let $\mathbf{Z}=[\mathbf{X} ; \mu]$, with Lagrange-multiplier $\mu$, then we need to find $\mathbf{Z}_{c}$ such that $\mathbf{\Phi}\left(\mathbf{Z}_{c}\right)=\mathbf{0}$, 
where $\boldsymbol{\Phi}(\mathbf{Z})$ is defined as

$$
\boldsymbol{\Phi}(\mathbf{Z}=[\mathbf{X} ; \mu])=\left[\begin{array}{c}
\nabla F_{i}(\mathbf{X})+\mu^{2} \nabla F_{j}(\mathbf{X}) \\
F_{i}(\mathbf{X})-F_{j}(\mathbf{X})
\end{array}\right] .
$$

This is a non-linear system of equations, which are all continuously differentiable. Therefore we can use a (damped) Newton's method with Jacobian

$$
J=\left[\begin{array}{cccc}
H_{i}^{11}+\mu^{2} H_{j}^{11} & H_{i}^{12}+\mu^{2} H_{j}^{12} & H_{i}^{13}+\mu^{2} H_{j}^{13} & 2 \mu \partial / \partial x F_{j} \\
H_{i}^{21}+\mu^{2} H_{j}^{21} & H_{i}^{22}+\mu^{2} H_{j}^{22} & H_{i}^{23}+\mu^{2} H_{j}^{23} & 2 \mu \partial / \partial y F_{j} \\
H_{i}^{31}+\mu^{2} H_{j}^{31} & H_{i}^{32}+\mu^{2} H_{j}^{32} & H_{i}^{33}+\mu^{2} H_{j}^{33} & 2 \mu \partial / \partial z F_{j} \\
\partial / \partial x\left(F_{i}-F_{j}\right) & \partial / \partial y\left(F_{i}-F_{j}\right) & \partial / \partial z\left(F_{i}-F_{j}\right) & 0
\end{array}\right],
$$

where $H_{i}^{l m}$ is the element on the $l$ th row and $m$ th column of the Hessian of the shape function of particle $i$. In more compact form, the Jacobian reads

$$
J=\left[\begin{array}{l:l}
\mathbf{H}_{i}+\mu^{2} \mathbf{H}_{j} & 2 \mu \nabla F_{j} \\
\hdashline\left(\nabla F_{i}-\nabla F_{j}\right)^{T} & 0
\end{array}\right] .
$$

This is implemented in MercuryDPM in the method

SuperQuadric: : get JacobianOfContactDetectionObjective. Furthermore, $\boldsymbol{\Phi}$, is computed in SuperQuadric : : computeResidualContactDetection.

Given an initial point $\mathbf{Z}^{0}=\left[\mathbf{X}^{0} ; \mu^{0}\right]$ and tolerance $\epsilon \ll 1$, we can then find the point $\mathbf{Z}_{c}$ that (almost) satisfies $\boldsymbol{\Phi}\left(\mathbf{Z}_{c}\right)=\mathbf{0}$ using a damped Newton method with damping factor $s \in(0,1]:$

0 . Set $s=1$ and $n=0$

1. Compute $J\left(\mathbf{Z}^{n}\right)$ and $\boldsymbol{\Phi}\left(\mathbf{Z}^{n}\right)$ and solve $\delta \mathbf{Z}$ from $J \delta \mathbf{Z}=-\boldsymbol{\Phi}$

2. Update $\mathbf{Z}^{n+1}=\mathbf{Z}^{n}+s \delta \mathbf{Z}$

3. If $\left|\boldsymbol{\Phi}\left(\mathbf{Z}^{n+1}\right)\right|<\epsilon$, set $\mathbf{Z}_{c}=\mathbf{Z}^{n+1}$ and stop.

4. If $\left|\boldsymbol{\Phi}\left(\mathbf{Z}^{n+1}\right)\right|<\left|\boldsymbol{\Phi}\left(\mathbf{Z}^{n}\right)\right|$, set $s=1$, if $\left|\boldsymbol{\Phi}\left(\mathbf{Z}^{n+1}\right)\right| \geq\left|\boldsymbol{\Phi}\left(\mathbf{Z}^{n}\right)\right|$, set $\mathbf{Z}^{n+1}=\mathbf{Z}^{n}$ and $s=s / 2$.

Go back to step 1 with $n=n+1$.

After these iterations, we find the (approximate) contact-point $\mathbf{X}_{c}$ from $\mathbf{Z}_{c}=\left[\mathbf{X}_{c} ; \mu_{c}\right]$.

This procedure is implemented in MercuryDPM in SuperQuadric : : computeContactPoint.

\section{FINDING INITIAL GUESS FOR CONTACT DETECTION}

In the procedure in last section, we need an initial point $\mathbf{Z}^{0}$ before starting the iterationprocedure. If we have an existing interaction, we take the contact-point and Lagrange multiplier of the last time-step as our initial guess. If not, we take the contact point of the bounding spheres of both particles, and a Lagrange multiplier-value of $\mu=1$.

However, sometimes this initial guess is too far from the actual contact-point for the damped Newton method above to converge. What we do then, is to start with the two bounding spheres for both particles, and slowly adapt the shape to the superquadric 
particle, using the contact points of the intermediate shapes as initial point for the next step. So for two particles $i, j$ with shapes $\left(a_{i, j}, b_{i, j}, c_{i, j}, \epsilon_{1 ; i, j}, \epsilon_{2 ; i, j}\right)$ and bounding radii $r_{\text {bounding; } i, j}$, we define $a_{i, j}^{k}, b_{i, j}^{k}, c_{i, j}^{k}, \epsilon_{1 ; i, j}^{k}, \epsilon_{2 ; i, j}^{k}$ as

$$
\begin{aligned}
a_{i, j}^{k} & =r_{\text {bounding } ; i, j}+k \frac{a_{i, j}-r_{\text {bounding; } i, j}}{n}, \\
b_{i, j}^{k} & =r_{\text {bounding; } i, j}+k \frac{b_{i, j}-r_{\text {bounding } ; i, j}}{n}, \\
c_{i, j}^{k} & =r_{\text {bounding } ; i, j}+k \frac{c_{i, j}-r_{\text {bounding } ; i, j}}{n}, \\
\epsilon_{1 ; i, j}^{k} & =1+k \frac{\epsilon_{1 ; i, j}-1}{n}, \\
\epsilon_{2 ; i, j}^{k} & =1+k \frac{\epsilon_{2 ; i, j}-1}{n},
\end{aligned}
$$

where $k=0 . . n$ is the stage number, and $n$ is the number of stages to go from a sphere to the superquadric particle.

For each stage, we compute the contact point. The first stage, $k=0$, is the interaction between the bounding spheres. For this we use the contact-detection of spheres, which gives us contact point $\mathbf{Z}_{0}$. For each subsequent stage, we compute the contact-point between particles with geometries $\left(a_{i, j}^{k}, b_{i, j}^{k}, c_{i, j}^{k}, \epsilon_{1 ; i, j}^{k}, \epsilon_{2 ; i, j}^{k}\right)$, using $\mathbf{Z}_{k-1}$ as initial point. In the end, at stage $n$, we then find the contact-point $\mathbf{Z}_{c}=\mathbf{Z}_{n}$. For more details, see page 106 of [6].

The implementation of this procedure can be found in SuperQuadric: : getContactPointPlanB. This way, for each stage the contact point for the temporary particles is closer to the actual contact point than if we would go from sphere to superquadric in one step. The number of stages $n$ from spheres to actual particles is adaptive as well: we first try it with 4 steps, and double the number of steps twice more if the contact detection still diverges.

The entire procedure for getting an initial guess for the contact-point is implemented in MercuryDPM in SuperQuadric: : getInitialGuessForContact.

\section{Particle-wall contact detection}

FLAT, INFINITE WALL

Instead of only particle-particle interactions, we are also interested in contacts between particles and walls. The easiest system is a flat, infinite wall. Given a wall with inward normal unit-vector $\mathbf{N}_{w}$ and any point on the wall $\mathbf{X}_{w}$ in the lab-fixed coordinate system, the points on the wall are given by the solutions of $\mathbf{N}_{w} \cdot\left(\mathbf{X}-\mathbf{X}_{w}\right)=0$. We follow [6], and look for the point where the particle penetrates the wall the furthest, in terms of the (particle) body-fixed coordinates (with $\mathbf{n}=A^{T} \mathbf{N}_{w}$ ):

maximise $\mathbf{n} \cdot \mathbf{x}$,

subject to $f(\mathbf{x})=0$. 
This system has been solved by [6], with the solution $\mathbf{x}=(x, y, z)$ where

$$
\begin{aligned}
& x=\frac{a \operatorname{sign}\left(n_{x}\right)}{\left(\left(1+\alpha^{2 / \epsilon_{2}}\right)^{\epsilon_{2} / \epsilon_{1}}+\beta^{2 / \epsilon_{1}}\right)^{\epsilon_{1} / 2},} \\
& y=\frac{\alpha b}{a}|x| \operatorname{sign}\left(n_{y}\right), \\
& z=\frac{\beta c}{a}|x| \operatorname{sign}\left(n_{z}\right), \\
& \alpha=\left|\frac{b n_{y}}{a n_{x}}\right|^{\epsilon_{2} / 2-1}, \\
& \beta=\left(\left(1+\alpha^{2 / \epsilon_{2}}\right)^{\epsilon_{2} / \epsilon_{1}-1}\left|\frac{n_{z} c}{n_{x} a}\right|\right)^{\epsilon_{1} /\left(2-\epsilon_{1}\right)} .
\end{aligned}
$$

Note, that this gives problems when $n_{x}=0$. In that case, we realise that $(x, a)$ and $(y, b)$ are interchangeable for a superquadric particle, so we then compute

$$
\begin{aligned}
& x=0, \\
& y=\frac{b \operatorname{sign}\left(n_{y}\right)}{\left(1+\beta^{2 / \epsilon_{1}}\right)^{\epsilon_{1} / 2},} \\
& z=\frac{\beta c}{b}|y| \operatorname{sign}\left(n_{z}\right), \\
& \beta=\left(\left|\frac{n_{z} c}{n_{y} b}\right|\right)^{\epsilon_{1} /\left(2-\epsilon_{1}\right)} .
\end{aligned}
$$

In case both $n_{x}=0$ and $n_{y}=0$, the solution is simply $x=y=0, z=c \operatorname{sign}\left(n_{z}\right)$. This is implemented in InfiniteWall: :getFurthestPointSuperQuadric and tested for ellipsoids in SuperQuadricDemos/UnitTests/ContactWithWallUnitTest. It is not tested yet for generic convex superquadric particles.

\section{Cylindrical DRUM}

For a circular drum with the centre at the origin and the axis in z-direction. We assume the drum has zero curvature at the contact point, and follow the procedure of contact detection for an InfiniteWall in 5.A.2. Given a particle at position $\mathbf{X}_{0}=\left(X_{0}, Y_{0}, Z_{0}\right)$, and drum with radius $r$, we say that the normal to the InfiniteWall is $\left(X_{0}, Y_{0}, 0\right)$ (since the centre of the drum is at the origin), and contact point is $\mathbf{X}_{c}=\left(\tilde{r} X_{0}, \tilde{r} Y_{0}, Z_{0}\right)$, where $\tilde{r}=r / \sqrt{X_{0}^{2}+Y_{0}^{2}}$. The zero-curvature approximation is valid if the drum is much larger than the particles, but does introduce some error when the curvature of the wall is very large, as the linearisation then gives a significant error. The more elongated the particle is, the bigger the problem, as the contact-point may be further away from the point the wall is linearised around. For an example what a simulation could look like with this type of wall, see figure 5.A.2.

The class-structure for this wall is not straightforward. Currently, the circular drum is implemented in Walls/SimpleDrumSuperquadrics, derives from BaseWall and contains both a radius and a mutable Inf initeWall. This Inf initeWall must be mutable, 


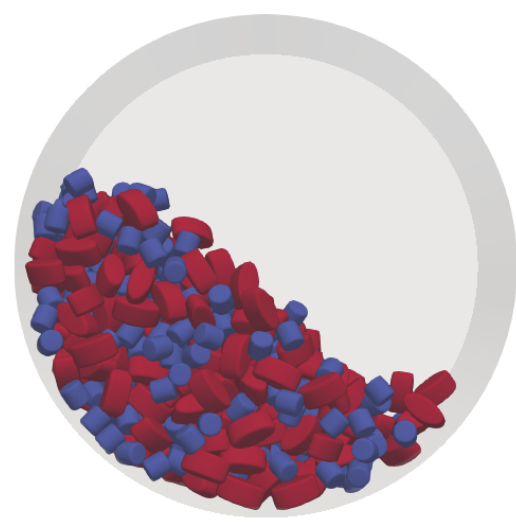

Figure 5.A.2: Example of superquadric particles in a rotating drum. Both particle types have blockiness parameters $\epsilon_{1}=0.1, \epsilon_{2}=1$, the blue and red particles have axis-lengths $a=b=c$ and $a>b=c$, respectively.

since its normal and position change with every contact-detection step, which uses the const-methods getDistanceAndNormal or getDistanceAndNormalSuperquadric. As an alternative, one could remove the const from these methods (which changes the interface for all walls, and is therefore not desirable), or const-cast the InfiniteWall in these methods. It is also possible to derive SimpleDrumSuperquadrics from InfiniteWall instead of using composition, but then again the methods mentioned above cannot be const anymore.

\section{A.3. FORCE COMPUTATION}

\section{NORMAL AND OVERLAP}

Once it is determined that two particles or a particle and wall are in contact with each other, the forces resulting from these contacts are computed. For the interaction between two particles $i$ and $j, i<j$, we compute the normal direction $\mathbf{n}_{i j}$ at the contactpoint as the direction of the gradient of the shape function at the contact-point $\mathbf{X}_{c}$ as

$$
\begin{aligned}
& \mathbf{n}_{i j}=\frac{\nabla_{\mathbf{X}} F_{i}\left(\mathbf{X}_{\mathbf{c}}\right)}{\left|\nabla_{\mathbf{X}} F_{i}\left(\mathbf{X}_{\mathbf{c}}\right)\right|}, \\
&\left(=-\frac{\nabla_{\mathbf{X}} F_{j}\left(\mathbf{X}_{\mathbf{c}}\right)}{\left|\nabla_{\mathbf{X}} F_{j}\left(\mathbf{X}_{\mathbf{c}}\right)\right|}\right) .
\end{aligned}
$$

This normal vector points from particle $i$ to particle $j$. There is no separate method in MercuryDPM to compute this value, as it is just the gradient normalised.

Defining $\delta_{i, j}^{n}$ as the distance between the contact point and surface, in normal direction, for particles $i$ and $j$ respectively, we compute the overlap $\delta_{i j}^{n}=\delta_{i}^{n}+\delta_{j}^{n}$ [6]. So we 
need to find $\delta_{i}^{n}>0, \delta_{j}^{n}>0$ such that

$$
\begin{aligned}
& F_{i}\left(\mathbf{X}_{c}+\delta_{i}^{n} \mathbf{n}_{i j}\right)=0, \\
& F_{j}\left(\mathbf{X}_{c}-\delta_{j}^{n} \mathbf{n}_{i j}\right)=0 .
\end{aligned}
$$

The computation of $\delta_{i, j}$ is done in MercuryDPM with a damped Newton method in the function SuperQuadric : : overlapFromContactPoint, where the initial guess $\delta_{i}=$ $\delta_{j}=0$ is used.

To compute the tangential overlap between the particles, we follow [11]. First, we use the usual condition of the tangential overlap as the integrated tangential velocity:

$$
\frac{d \boldsymbol{\delta}_{i j}^{t}}{d t}=\mathbf{v}_{i j}^{t},
$$

where the tangential velocity $\mathbf{v}_{i j}^{t}$ is the tangential component of the relative velocity of particles $i$ and $j$. Furthermore, we correct the tangential overlap for rotations of the contact every time-step; every time-step we subtract the normal component and make sure we keep the length the same:

$$
\begin{aligned}
\tilde{\boldsymbol{\delta}}_{i j}^{t} & =\boldsymbol{\delta}_{i j}^{t}-\left(\boldsymbol{\delta}_{i j}^{t} \cdot \mathbf{n}_{i j}\right) \mathbf{n}_{i j}, \\
\boldsymbol{\delta}_{i j}^{t} & =\tilde{\boldsymbol{\delta}}_{i j}^{t} \frac{\left|\boldsymbol{\delta}_{i j}^{t}\right|}{\left|\tilde{\boldsymbol{\delta}}_{i j}^{t}\right|}
\end{aligned}
$$

Note, that the second order correction of Weinhart et al. [12], who use $\frac{\mathrm{d} \boldsymbol{\delta}_{i j}^{t}}{\mathrm{~d} t}=\mathbf{v}_{i j}^{t}-\frac{\left(\boldsymbol{\delta}_{i j}^{t} \cdot \mathbf{v}_{i j}\right) \mathbf{n}_{i j}}{r_{i j}}$, cannot be used, since the vector connecting the centres of the particles is generally different from the normal direction of the interaction.

For the normal of a particle-wall contact, we simply take the normal of the wall as the normal of the interaction. The overlap is then $\delta_{i}^{n}$ as computed in (5.57), and the tangential overlap is given by (5.59), without any extra correction.

\section{CONTACT MODEL}

Using the overlap and the normal of a contact, the contact-force can be computed. For the LinearViscoElasticNormalInteraction, nothing needed to be changed. For the HertzianViscoelasticInteraction, we compute the effective radius following [6], using the mean local curvature coefficient $r_{\text {eff: }}$ :

$$
\begin{aligned}
K & =\frac{\nabla F^{T} \mathbf{H} \nabla F-|\nabla F|^{2} \operatorname{trace}(\mathbf{H})}{2|\nabla F|^{3}}, \\
r_{\text {eff }} & =1 / K,
\end{aligned}
$$

where $F$ is the shape-function of the particle and $\mathbf{H}$ is the Hessian of the shape-function. We can then use the Hertzian contact-model without any further changes.

The corrections (5.60) - (5.61) are implemented in MercuryDPM in the linear friction models, namely FrictionInteraction and SlidingFrictionInteraction.

\section{A.4. OUTLOOK}

Although we managed to get the superquadric particles working in MercuryDPM in combination with some basic building blocks, a lot still remains to be done. The hardest 
part here would be the interaction between superquadric particles and various kinds of walls. MercuryDPM contains many types of complex walls, and it is not straightforward how to correctly compute the interactions. Generally, if the curvature of the wall is very low, it can be linearised and the procedure of the infinite flat wall can be followed, just like is done with the circular drum. Other walls might get triangulated, however, the corners might form a problem here. One could try a level-set of some kind for these walls, but one has to think of both the scaling of this level-set and be careful of sharp corners; here the derivative of the level-set is not defined and therefore something like a Newton's method cannot be used. Rounding the corners with spheres is also not an option, as it would need different amounts of curvatures if four or more walls come together at incompatible angles.

Furthermore, the different kinds of particle species and interaction-types should be made compatible with superquadric particles; this is straightforward for some interactions, but harder for others like the interactions concerned with liquid bridges or sintering. It is also very important that not only the interactions are adapted to be able to cope with superquadric particles, but they should also be validated in those cases.

Lastly, the performance of the code is poor at the moment. Everything is implemented naively, and without the possibility for parallelisation of a simulation. We are therefore currently bound by simulations of at most a few thousand particles, which is much smaller than industrial processes would need. However, the code is already usable for small-scale scientific problems, as is shown earlier in this chapter.

\section{B. DRUM-ALGORITHM}

First, we summarise the different stages of the drum simulation used in chapter 5 . Secondly, we give the equations for rotating the particles fixed to the rotating drum wall.

\section{B.1. DRUM SIMULATION}

To simulate a rotating drum of radius $r$ and width $w$, filled with a bidisperse mixture of spherical and ellipsoidal particles, we follow the following procedure:

1. Drum creation: populate a flat inclined plane of length $2 \pi r$ with particles (ChuteBottom in MercuryDPM, see also [12]), and move all particles to a position as if the bottom is wrapped around the origin with radius $r: x_{\text {new }}=(r+z) \cos (x), y_{\text {new }}=y$ and $z_{\text {new }}=(r+z) \sin (x)$. Go to step 2 .

2. Fill the drum with the desired mixture of particles using random locations, so that the particles do not touch each other or the drum. If we can insert the desired number of particles, go to step 4. Otherwise, if we fail to insert a particle 1000 times at different locations, stop inserting and go to step 3.

3. Start the time evolution, and let the particles settle. Once the particles are sufficiently settled, go back to step 2 .

4. Start the time evolution, and let the particles settle. Once the particles are sufficiently settled, start the rotation of the drum (see 5.B.2). 
Steps 1 and 2 are performed before the first time step, and step 2 is done in between time steps. Steps 3 and 4 both require regular DPM time-stepping.

\section{B.2. PRESCRIBED POSITION AND VELOCITY}

In order for the drum to rotate with angular velocity $(0, \omega, 0)$, we have to prescribe the position, velocity and angular velocity of the fixed drum-particles. Since we centre the drum around the origin, we can compute the new velocity and position of each fixed particle $i$ as follows:

- Compute the distance from the origin, $r_{i}=\sqrt{x_{i}^{2}+z_{i}^{2}}$, and the angle between the $\mathrm{x}$-axis and the position vector, $\theta_{i}=\tan ^{-1}\left(z_{i} / x_{i}\right)$ (use atan2 for this).

- Compute the new angle as $\theta_{i} \leftarrow \theta_{i}+\omega \Delta t$

- Move the particle $i$ to position $\mathbf{X}_{i}=\left(x_{i}, y_{i}, z_{i}\right)=\left(r_{i} \cos \theta_{i}, y_{i}, r_{i} \sin \theta_{i}\right)$ and set its velocity to $\mathbf{U}_{i}=\left(u_{i}, v_{i}, w_{i}\right)=\left(-\omega r_{i} \sin \theta_{i}, 0, \omega r_{i} \cos \theta_{i}\right)$

- Set the angular velocity of all the fixed particles to $\omega$.

\section{REFERENCES}

[1] MercuryDPM, https : //www . mercurdpm . org, last accessed on August 27th 2019.

[2] A. R. Thornton, T. Weinhart, S. Luding, and O. Bokhove, Modeling of particle size segregation: calibration using the discrete particle method, International Journal of Modern Physics C 23, 1240014 (2012).

[3] T. Weinhart, A. R. Thornton, S. Luding, and O. Bokhove, From discrete particles to continuum fields near a boundary, Granular Matter 14, 289 (2012).

[4] T. Weinhart, M. Post, I. F. C. Denissen, D. R. Tunuguntla, E. Grannonio, N. Losacco, J. Barbosa, W. K. Den Otter, and A. R. Thornton, Fast, flexible particle simulations: An introduction to MercuryDPM, in Proceedings of the 8th International Conference on Discrete Element Methods (2019).

[5] A. Jaklic, A. Leonardis, and F. Solina, Segmentation and recovery of superquadrics, Vol. 20 (Springer Science \& Business Media, 2013).

[6] A. Podlozhnyuk, S. Pirker, and C. Kloss, Efficient implementation of superquadric particles in discrete element method within an open-source framework, Computational Particle Mechanics 4, 101 (2017).

[7] G. Lu, J. R. Third, and C. R. Müller, Discrete element models for non-spherical particle systems: from theoretical developments to applications, Chemical Engineering Science 127, 425 (2015).

[8] A. H. Barr, Superquadrics and angle-preserving transformations, IEEE Computer graphics and Applications 1, 11 (1981). 
[9] A. R. Thornton, T. Weinhart, V. Ogarko, and S. Luding, Multi-scale methods for multi-component granular materials, Comp. Meth. Mat. Sci 13, 197 (2013).

[10] D. Krijgsman, V. Ogarko, and S. Luding, Optimal parameters for a hierarchical grid data structure for contact detection in arbitrarily polydisperse particle systems, Computational particle mechanics 1, 357 (2014).

[11] S. Luding, Cohesive, frictional powders: contact models for tension, Granular matter 10, 235 (2008).

[12] T. Weinhart, A. R. Thornton, S. Luding, and O. Bokhove, Closure relations for shallow granular flows from particle simulations, Granular Matter 14, 531 (2012). 


\section{6}

\section{CONCLUSIONS AND OUTLOOK}

The aim of this thesis was to develop more accurate and efficient models to predict and understand granular flows. Granular flows differ from Newtonian, classical fluid flows in two important aspects: (a) their rheology is highly nonlinear, with viscosity diverging at zero shear rate and vanishing at zero pressure; and (b) granular flows are almost always mixtures of different granular components, and thus are prone to segregation. In this thesis, progressively more complex properties of granular flows have been modelled; starting by incorporating the granular rheology into a depth-averaged model (chapter 2), then moving on to size segregation (chapter 3/4), and shape segregation (chapter 5). In the following, the conclusions of previous chapters are elaborated on before an outlook and recommendations for further research are given.

In chapter 2, shallow monodisperse granular flows over a linearly contracting channel have been modelled. From experiments it is known that this type of flow can display a variety of steady flows, e.g. smooth sub- or supercritical flows and shocks in the flows entering the contraction [1]. We captured this behaviour with a (two-dimensional) depthaveraged granular flow model that is simulated with a discontinuous Galerkin finite element method using the in-house library hpGEM [2]. Going one step further, the depthaveraged model has also been width-averaged, resulting in a simple one-dimensional model that accurately predicts the width-averaged height and Froude number for both subcritical and supercritical flows. The supercritical flows have oblique shocks in the two-dimensional depth-averaged model that are not resolved in the one-dimensional width- and depth-averaged model. The latter model has been used to predict the flow structure in various regimes of inflow height and Froude number, where the width-averaged flow can either display a smooth subcritical profile, a smooth supercritical profile, or a profile with a standing shock in the contracting area. Lastly, we observed multiple states for certain Froude numbers and inflow heights, where the flow can display either a standing shock or a smooth profile.

In chapter 3, we looked at another experimentally known phenomenon, namely the bulbous head formation in segregating flows down a long, narrow channel [3]. While there are multiple possible effects that can cause the bulbous head formation $[4,5]$, here 
we focused purely on the effect of size segregation. Using a depth-averaged continuum model, it has been shown with numerical simulations that the bulbous head structure naturally emerges as long as the large-particle phase is more frictional than the smallparticle phase. Furthermore, we have shown an analytical solution with a combination of a novel travelling wave solution for bidisperse flows, and a travelling wave solution for the large-particle phase. Lastly, the bulbous head formation has also been shown in full three-dimensional discrete particle simulations performed using MercuryDPM [6-9]. A major problem for comparing discrete particle simulations with the results of the continuum model, is to generate the correct inflow condition. For this, we have developed a so-called maser inflow boundary condition, that uses a small periodic flow to generate a segregated inflow of particles with constant flow height. Since there is feedback from the front of the flow, the influx of particle mass is unfortunately not constant for subcritical flows, but decreases over time. Comparing the discrete particle simulations with the calibrated one-dimensional model for two test cases with different inflow heights, we have seen that the agreement is surprisingly good for the thicker flow, but less so for the very thin flow. We hypothesised that this disagreement is mainly due to the fact that the thin flow is subcritical, for which other inflow boundary conditions should be used in the continuum model.

For very elongated granular flows studied in the first two chapters, the standard postprocessing techniques to get macroscopic data do either not conserve mass and momentum, or the resulting fields do not represent the stretched coordinate system that is necessary in the depth-averaged continuum model. Thus in chapter 4 , we have developed a micro-macro technique called anisotropic coarse graining, which can obtain continuum fields from the discrete particle data with different length scales in different directions, while still being grid independent and conserving both mass and momentum. This is especially helpful in shallow flows with elongated flow features, such as breaking segregation waves or subtle downslope segregation patterns. Here, we chose our coordinate system for the discrete particle simulations such that it has the same direction as the system features, but one could choose to scale the coordinate system for the coarse grained fields in any direction.

Applying the anisotropic coarse graining technique to bidisperse shallow granular flows over a rough channel, we have seen that microscopic friction is a cause of segregation in these flows. Furthermore, the sharpness and length of breaking segregation waves depends on the size ratio of the particles and height of the flow: the breaking segregation wave becomes sharper and narrower with increasing ratio of particle sizes and decreasing flow height.

Lastly, in chapter 5 we looked at the influence of particle shape on segregation. For this purpose, we have implemented elliptic particle shapes and their contact detection in MercuryDPM. We then used a rotating drum to simulate binary mixtures of spheres and prolate ellipsoids of different aspect ratios. We have seen that the segregation due to differences in aspect ratio can change direction: for mixtures of spheres and prolate ellipsoids with a small aspect ratio, the ellipsoids segregate to the core of the flow. For the mixtures of spheres and more elongated prolate ellipsoids, the ellipsoids tend to segregate towards the outside. This indicates that the direction of segregation for ellipsoids of equal density and volume as the spheres is not monotonically dependent on the sin- 
gle shape parameter of the ratio of long and short ellipsoid axes, which implies that a) at least two segregation mechanisms are at play here and b) a continuum model for shapebased segregation is not easy to achieve.

\section{OUTLOOK}

In the future, it should be tested under what conditions the models of chapters 2 and 3 are in agreement with discrete particle simulations and experiments of these systems. It is recommended to compare the continuum model results with data of a large variety of both discrete particle simulations and experiments, to validate the modelling assumptions made. There are currently a very large number of continuum models, and such a database would make it possible to test which models perform best in which situations. One could then also model more goal oriented: it would be clear for which flows the existing models would not be good enough, and which modelling assumptions should thus be relaxed.

Furthermore, there will always be systems where efficient continuum models are not able to reliably predict the behaviour of granular flows, but particle simulations are too expensive. For these cases, one should attempt to find multiscale models, where parts of the simulations can be done with efficient continuum models, and parts can be done with particle based simulation methods. Currently, the relevant length scales of the interesting features in the discrete particle simulations are determined by hand, which is tedious and time-consuming. In order to successfully use anisotropic coarse graining in a multiscale model, one should automate the process of finding the relevant length scales, especially if they may change over time.

Granular materials in the real world are rarely uniform in size, shape, surface roughness, and other properties. Current research shows that it is not easy to predict even the direction of segregation when using ellipsoidal particles, let alone the direction and strength of segregation of more complex shaped particles. Similarly, we do not know whether the segregation due to a lack of uniformity of the particles is strong enough to be problematic in industrial processes, or whether these distributions can safely be ignored in particle based and continuum models. Especially properties that only weakly induce segregation might be ignored in some systems, but not others. With increasing possibilities for particle based simulations, clear guidelines for incorporating the various effects should be developed.

Lastly, the heavy use of particle based simulation techniques is wonderful because we can obtain a lot of data out of it. On the other hand, we have to be careful since the conclusions we draw from these simulations are only as good as how well they represent reality. In order to make both accurate and efficient predictions of granular flows, one should use a combination of various types of experiments, particle based models and continuum models instead of relying on just one or two of those. Finding a method to incorporate all these different elements is one of the main challenges for future research.

\section{REFERENCES}

[1] A. Vreman, M. Al-Tarazi, J. Kuipers, M. Van Sint-Annaland, and O. Bokhove, Supercritical shallow granular flow through a contraction: experiment, theory and simula- 
tion, Journal of Fluid Mechanics 578, 233 (2007).

[2] L. Pesch, A. Bell, W. Sollie, V. Ambati, O. Bokhove, and J. van der Vegt, $h p G E M-A$ software framework for discontinuous Galerkin finite element methods, ACM Transactions on Mathematical Software 33(4) (2007).

[3] R. M. Iverson, M. Logan, R. G. LaHusen, and M. Berti, The perfect debris flow? Aggregated results from 28 large-scale experiments, Journal of Geophysical Research: Earth Surface 115 (2010).

[4] B. P. Kokelaar, R. L. Graham, J. M. N. T. Gray, and J. W. Vallance, Fine-grained linings of leveed channels facilitate runout of granular flows, Earth and Planetary Science Letters 385, 172 (2014).

[5] R. Delannay, A. Valance, A. Mangeney, O. Roche, and P. Richard, Granular and particle-laden flows: from laboratory experiments to field observations, Journal of Physics D: Applied Physics 50, 053001 (2017).

[6] MercuryDPM, https : //www . mercurdpm. org, last accessed on August 27th 2019.

[7] A. R. Thornton, T. Weinhart, S. Luding, and O. Bokhove, Modeling of particle size segregation: calibration using the discrete particle method, International Journal of Modern Physics C 23, 1240014 (2012).

[8] T. Weinhart, A. R. Thornton, S. Luding, and O. Bokhove, From discrete particles to continuum fields near a boundary, Granular Matter 14, 289 (2012).

[9] T. Weinhart, M. Post, I. F. C. Denissen, D. R. Tunuguntla, E. Grannonio, N. Losacco, J. Barbosa, W. K. Den Otter, and A. R. Thornton, Fast, flexible particle simulations: An introduction to MercuryDPM, in Proceedings of the 8th International Conference on Discrete Element Methods (2019). 


\section{ACKNOWLEDGEMENTS}

This thesis symbolises the end of my journey towards a PhD. I did not travel alone, and I would like to take this opportunity to express my gratitude to the ones who accompanied me on this road.

Firstly, I would like to thank my promotor Anthony Thornton and co-promotor Thomas Weinhart. Anthony, I thank you for your enthousiasm and encouragement to finish my thesis. You also introduced me to software development, which I now love to do. I will never forget some of the nice trips we have made, in particular the trip to Santorini, which combined coding with fun and nature. Thomas, you have been the silent rock during my project; you were always there to support me with both the science and the writing, and your help, especially this past half year, was invaluable. Stefan, I thank you for our interesting scientific discussions and giving me a place in the Multiscale Mechanics group. Kees, thank you for your help during the final stage and convincing me again that science is fun. Your meetings always left me feeling happier afterwards.

During my PhD, I did not work alone. Deepak, I would like to thank you for our collaboration on both the flow through the contraction and the non-spherical particles. Even though our work-styles are opposites, we made it happen. Chris, thank you for giving me the need to develop the anisotropic coarse graining for your application. Nico, thanks for your useful remarks on the bulbous head paper.

$\mathrm{Na}$ al mijn jaren aan de Universiteit Twente associeer ik deze niet alleen met werk, maar ook met gezelligheid. Ik wil daarom ook graag al mijn collega's bedanken, zowel die van MSM als die van TFE die regelmatig bij de koffiepauzes aanwezig waren. In het bijzonder wil ik mijn dank uiten naar Marnix. Marnix, naast ons project hebben we ook lange tijd ons kantoor gedeeld. Ik vond het erg gezellig, en we hebben veel interessante discussies gehad over vanalles en nog wat. We hebben samen mooie tijden beleefd. Daarnaast wil ik mijn paranimf Bert bedanken, met wie ik jarenlang bijna iedere dag een lunchwandeling heb gemaakt. Jij wist altijd wat er speelde in de groep, en we hebben onze verbazing over menig gebeurtenis kunnen delen. Sylvia, jij was er altijd voor een praatje, en ik ben je erg dankbaar voor jouw al je hulp door de jaren heen. Wouter, bedankt voor het verhelpen van al mijn computerproblem en je aanstekelijke vrolijkheid.

Naast mijn collega's wil ik ook graag mijn vrienden bedanken. Vera, we hebben de afgelopen jaren lief en leed gedeeld, en ik vond het altijd erg gezellig om samen te klimmen en kletsen. Ik wens je veel succes met het afronden van je PhD. Ben en Remco, bedankt voor de gezelligheid tijdens het eten iedere vrijdagavond. Kemilly, bedankt voor het samen klimmen en het ontwerpen van de cover van mijn thesis. Ellen en Katinka, bedankt voor jullie steun tijdens moeilijke tijden. De klimmers bij Arqué en de muzikanten van Armonia wil ik bedanken voor alle gezellige avonden.

Papa, mama en Joris, jullie stonden altijd voor mij klaar en hebben me overal bij gesteund. Zonder jullie was ik niet zo ver gekomen.

Lieve Ruud, bedankt voor alles. 


\section{Curriculum Vitae}

\section{Irana Francisca Catharina Denissen}

06-04-1990 Born in Weststellingwerf, The Netherlands.

\section{EDUCATION}

2007-2010 B.Sc. in Applied Mathematics

University of Twente, The Netherlands

2010-2013 M.Sc. in Applied Mathematics

University of Twente, The Netherlands

Thesis: $\quad$ Discontinuous Galerkin finite element methods for the time-harmonic Maxwell equations in periodic media

Supervisor: $\quad$ Prof.dr.ir. J.J.W. van der Vegt

2014-2019 PhD candidate Multiscale Mechanics

University of Twente, The Netherlands

Thesis: $\quad$ On segregation in bidisperse granular flows

Promotor: $\quad$ Prof.dr. A.R. Thornton

Co-Promotor: Dr. T. Weinhart

\section{Publications}

\section{JOURNAL PUBLICATIONS}

1. I.F.C. Denissen, T. Weinhart, A. te Voortwis, S. Luding, J. M. N. T. Gray, A. R. Thornton, Bulbous head formation in bidisperse shallow granular flow over an inclined plane, Journal of Fluid Mechanics, 866 (2019).

2. T. Weinhart, L. Orefice, M. Post, M.P. van Schrojenstein Lantman, I.F.C. Denissen, D.R. Tunuguntla, J.M.F. Tsang, H. Cheng, M.Y. Shaheen, P. Rapino, E. Grannonio, N. Losacco, J. Barbosa, J.E. Alvarez Naranjo, W.K. den Otter, H. Shi, A.R. Thornton, Fast, flexible particle simulations - An introduction to MercuryDPM, submitted to Computer Physics Communications.

3. D.R. Tunuguntla, I.F.C. Denissen, T. Weinhart, J.J.W. van der Vegt, S. Luding, O. Bokhove, A.R. Thornton, Granular flow down a rough inclined channel with a linear contraction, in preparation. 


\section{Conference PROCEEDings}

1. T. Weinhart, M. Post, I.F.C. Denissen, D.R. Tunuguntla, E. Grannonio, N. Losacco, J.M.F. Tsang, J. Barbosa, W.K. den Otter, A.R. Thornton, Fast, flexible particle simulations: An introduction to MercuryDPM, 8th International Conference on Discrete Element Methods (2019).

2. T. Weinhart, D.R. Tunuguntla, M.P. van Schrojenstein Lantman, I.F.C. Denissen, C.R. Windows-Yule, H. Polman, J.M.F. Tsang, B. Jin, L. Orefice, K. van der Vaart, S. Roy, H. Shi, A. Pagano, W.M. den Breeijen, B.J. Scheper, A. Jarray, S. Luding, A.R. Thornton, MercuryDPM: Fast, flexible particle simulations in complex geometries part II: Applications, V International Conference on Particle-based Methods - Fundamentals and Applications (2017).

3. J. Torres Serra, D.R. Tunuguntla, I.F.C. Denissen, A. Rodríguez Ferran, E.E. Romero Morales, Discrete element modelling of granular column collapse tests with industrial applications, V International Conference on Particle-based Methods - Fundamentals and Applications (2017).

4. T. Weinhart, D.R. Tunuguntla, M.P. van Schrojenstein Lantman, A.J. van der Horn, I.F.C. Denissen, C.R. Windows-Yule, A.C. de Jong, A.R. Thornton, MercuryDPM: A fast and flexible particle solver part A: Technical advances, 7th International Conference on Discrete Element Methods (2016). 